\title{
A PROGRAMMING APPROACH TO THE NUMERICAL \\ ANALYSIS OF EIASTO-PLASTIC CONTINUA
}

\section{by}

\section{C.T. Dittmer M.Sc. (Eng.)}

A thesis submitted in fulfilment of the requirements

for the degree Doctor of Philosophy.

Department of Civil Engineering

University of Cape Town

June 1978

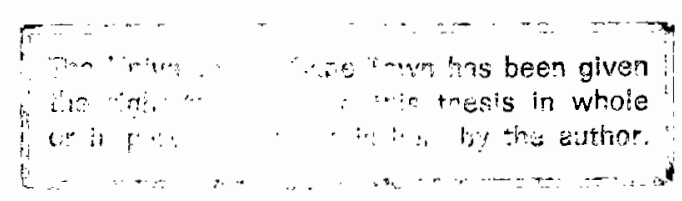


The copyright of this thesis vests in the author. No quotation from it or information derived from it is to be published without full acknowledgement of the source. The thesis is to be used for private study or noncommercial research purposes only.

Published by the University of Cape Town (UCT) in terms of the non-exclusive license granted to UCT by the author. 
ABSTRACT

The application of a kinematic minimum principle involving a continuous functional subject to inequality constraints is described for the incremental analysis of elasto-plastic continua. A simple algorithm is used for solution of the resulting mathematical programming problem. The formulation is presented for problems in plane stress, plane strain or axial symmetry, using triangular constant strain finite elements, and is extended to the use of cubic quadrilateral isoparametric elements for which a numerical integration technique is employed to account for elasto-plastic interfaces within elements. The material is assumed to obey the von Mises yield condition, and be either elastic-perfectly plastic or linear kinematic hardening. Computational details and solution techniques are described, and numerical examples compared with experimental and numerical results in the literature. Some assessment is made of the relative computational efficiency of the method. 


\section{DECLARATION OF CANDIDATE}

I hereby declare that this thesis is my own work and that it has not been submitted for a degree at any other university.

Signed by candidate

C.T. Dittmer
Signature Removed 


\section{ACKIJOWLEDGEMENTS}

I wish to express my thanks to the following:

Professor J.B. Martin, who supervised the research

AECI Ltd for generous financial support

Lee Behm for typing the manuscript

the operating staff of the Computer Centre at the University of Cape Town 
CHAPTER 1: Introduction

CHAPTER 2: $\quad$ Some Fundamental Concepts

2.1 Introduction 7

2.2 Equilibrium 7

2.3 Kinematic.relations 8

2.4 Constitutive relations 8

2.5 von Mises yield condition and linear
kinematic hardening

2.6 Plane stress 13

$\begin{array}{lll}2.7 & \text { Plane strain } & 15\end{array}$

$\begin{array}{lll}2.8 & \text { Axial symmetry } & 16\end{array}$

CHAPTER 3: The Kinematic Minimum Principle for the Rate Problem in Elasto-Plasticity

$\begin{array}{lll}3.1 & \text { Introduction } & 19\end{array}$

3.2 The classical rate problem in elasto-plasticity 19

3.3 Inversion of constitutive equations 21

3.4 The kinematic minimum principle for the rate problem

CHAPTER 4: Application of the Kinematic Minimum Principle to the Continuum Problem

4.1 Introduction 27

4.2 Application to constant strain finite elements 28 
4.3 An algorithm for the minimization of $\bar{U}_{p}^{0} \quad 39$

4.4 Reduction to tangent modulus approach 41

4.5 Solution procedure for the incremental problem 44

4.6 Application to higher order finite elements 47

CHAPTER 5: Solution Techniques, Uniqueness and Some Notes on the Computational Procedure

5.1 Introduction 58

5.2 Partitioning and triangular decomposition 60

5.3 Gauss elimination 63

5.4 Relative computational efficiency 64

5.5 Uniqueness of solution in elastic-perfectly
plastic case

5.6 Load increment magnitude 71

$\begin{array}{lll}5.7 & \text { Determination of } V_{p} & 73\end{array}$

5.8 Correction of stresses in $V_{p}$ for elastic-perfectly plastic case

CHAPTER 6: Numerical Examples

6.1 Introduction 75

6.2 Vee-notched tension specimen 75

6.3 Deep cantilever in plane stress 92

6.4 Spherical pressure vessel-flush nozzle junction 103

$\begin{array}{lll}\text { CHAPTER 7: } \quad \text { Discussion and Conclusions } & 108\end{array}$

REFERENCES 
APPENDIX Computer Programs and User Manuals

A.1 General description A-1

A.2 Storage allocation, dimensioning of arrays,
and execution

A.3 Program EPTCS

3.1 Data input for EPTCS A-6

3.2 Description of data input A-7

3.3 Example of data and results for EPTCS A-11

A.4 Program EPCQI

4.1 Data input for EPCQI A-23

4.2 Description of data input A-24

4.3 Example of data and results for EPCQI A-27

A.5 EPCTS program listing A-39

A.6. EPCQI program listing A-70 


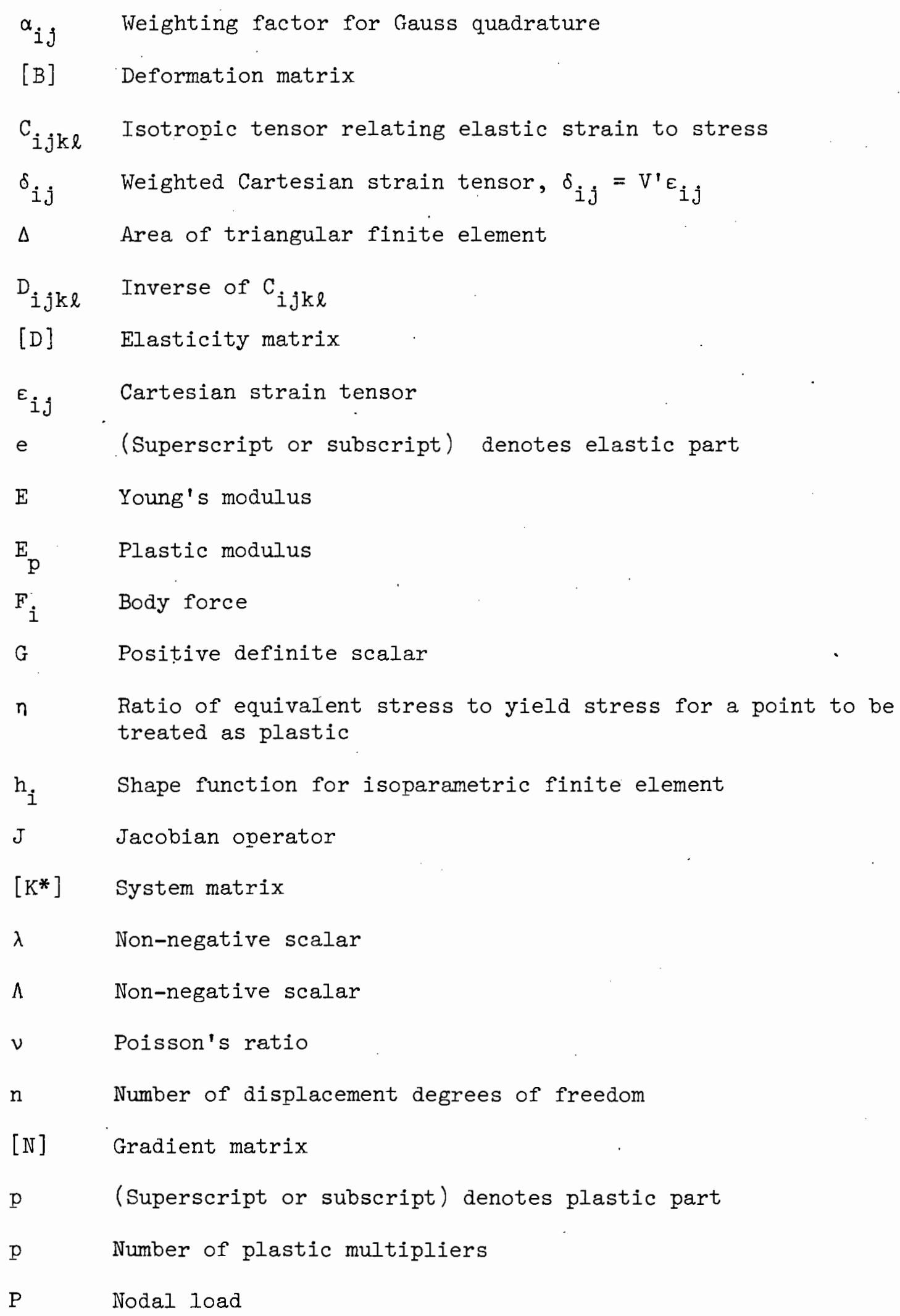




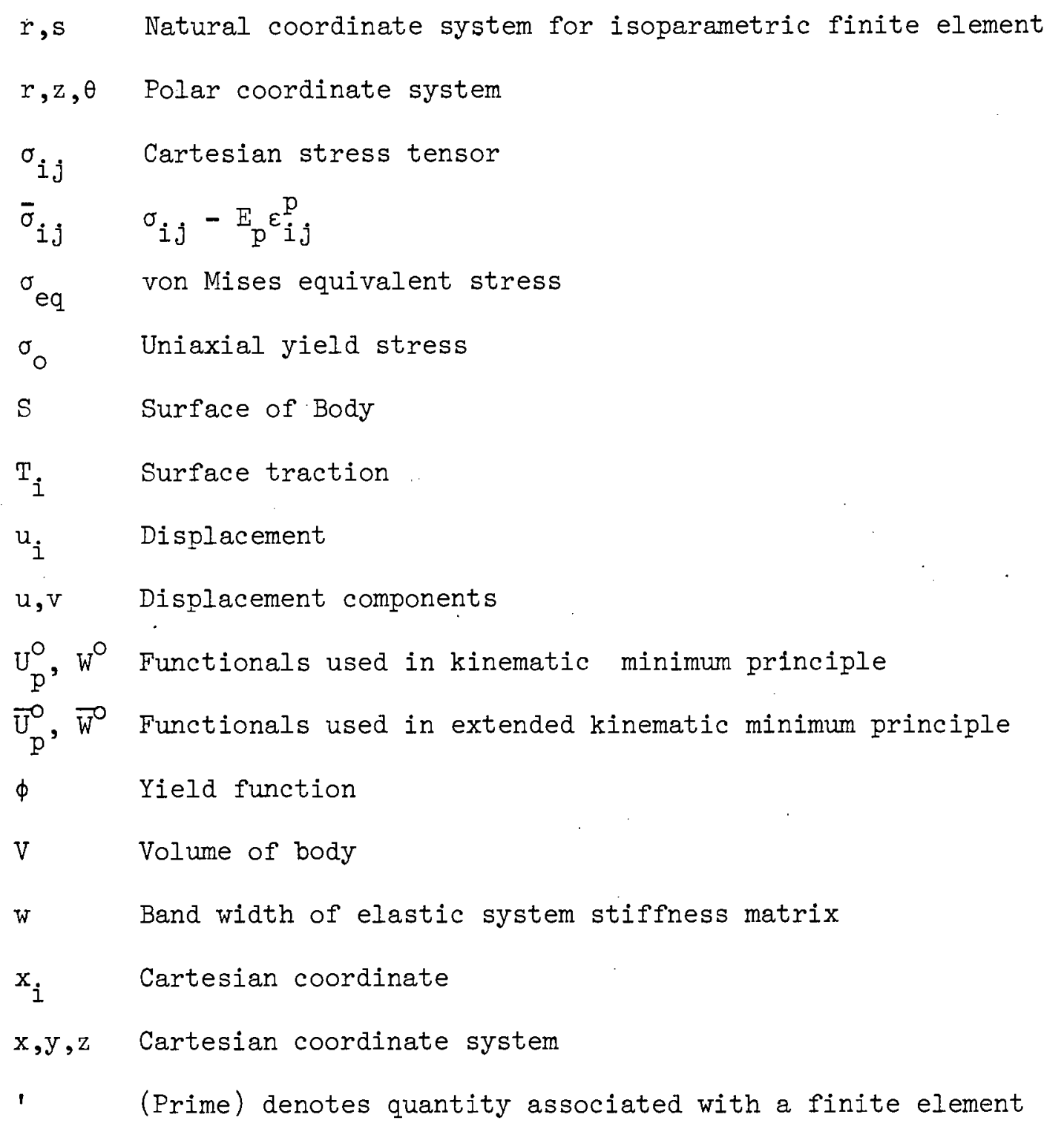




\section{H A P T E R 1}

INTRODUCTION

The simplicity of the laws governing the mechanical behaviour of elastic solids in many cases permits an analytical solution. In elastic problems of greater complexity with regard to geometry, boundary conditions, loading or nonhomogeneous material properties, recourse is generally made to numerical solutions which exploit the minimum principles governing the mechanical behaviour of elastic solids. In particular the finite element method has been extensively used to determine approximate solutions by discretizing the description of the spatial field to a finite number of parameters.

For elasto-plastic solids the complexity of mechanical behaviour generally prohibits an analytical solution. Although the minimum principles governing the mechanical behaviour of elasto-plastic solids have been established for some time (Prager [1], [2]; Hodge and Prager [3]; Greenberg [4], [5] and Koiter [6]), it was only with developments in numerical methods that their exploitation become feasible. More recently Ceradini [7] and Maier [8] have given alternative forms for the minimum principles using elastic solutions for residual fields. Maier's kinematic minimum principle was derived from quadratic programming arguments.

Direct methods of elasto-plastic analysis which use the minimum principles and the finite element method include initial strain/initial stress and tangent modulus approaches. In the initial strain method deviloped by Galagher, Padlog and Bijlaard [9], and Argyris [10], plastic 
strains during a load increment are treated as initial strains, the system stiffness matrix remaining elastic and unchanged. This iterative procedure fails for an elastic-perfectly plastic material as plastic strain increments are not uniquely described. The initial stress approach of Zienkiewicz, Valliapan and King [11] entails the iterative elastic distribution of 'initial stresses' until the requirements of equilibrium, the kinematic relations and constitutive laws are satisfied, again the system matrix remaining unchanged. Since the stress distribution is uniquely described by increments of strain, ideal plasticity can be accommodated. For the initial strain/initial stress methods the system matrix need be inverted once only, however the number of iterations required for convergence at each load increment may increase as plastic strain increments become larger. In these methods elastic unloading is automatically accommodated as the system matrix always reflects elastic stress-strain relations.

The tangent modulus or variable stiffness method of Pope [12], Swedlow [13], Marcal and King [14], and Yamada, Yoshimura and Sakurai [15] requires reformulation of the system matrix at each stage in the incremental loading procedure, taking account of adjustment to stress-strain relations due to plastic strains. Further, iteration is required for any load increment in which elastic unloading occurs as the system matrix must reflect the true stress-strain relations. This method can be used for perfectly plastic materials. Marcal [16], in comparing the two methods, derived the initial strain formulation from the tangent modulus approach. More recently efforts have been directed towards formulating elasto-plastic problems as formal mathematical programming problems and to use standard programming techniques to determine a solution. Some examples are the work of Hodge, Belytscho and Herakovich [17], 
Sayegh and Rubenstein [18], Giacomini, Maier and Paterlini [19], de Donato and Maier [20], and Anand and Garg [21]. As shown by de Donato and Franchi [22] a linear complementary problem emerges which is fully equivalent to two dual quadratic programming problems. The solution can be determined from any one of the five formulations. However, in applying nonlinear programming algorithms, the size of matrices to be calculated for any of the formulations is prohibitive even for small numbers of finite elements. The most efficient formal programming technique for incremental elasto-plastic analysis appears to be the 'multistage loading' and 'reduced problem technique' used by de Donato and Franchi [22] and de Donato and Maier [20]. For this the yield surface is piecewise linearized, transforming the domain of permissible stress states into a series of linear inequalities. Since a plastic multiplier and linear inequality are associated with each yield plane the numbers of variables and constraints increases rapidly. 'Multistage loading' consists of the initial division of the load into a given number of 'sub-loads' each of which is increased from zero to its final value. Elastic unloading can only be considered at the beginning of each loading stage. To decrease the large number of variables the 'reduced problem technique' is employed in which yielding modes that appear unlikely to be activated are omitted from that stage of the problem, and any standard nonlinear programming technique used to determine the solution. Violation of ignored constraints necessitates iteration. To the writer's knowledge there is an absence in the literature of numerical applications of the formal mathematical programming approach to three-dimensional continuum problems, which though conceptually quite feasible, must present a formidable computational task.

In investigating Maier's theorem [8] derived from quadratic programming arguments, Martin [23] has given a simpler result in a 
kinematic minimum principle for the rate problem in elasto-plasticity, involving a continuous functional subject to inequality constraints. This was applied in incremental form to the plane truss problem by Martin and Reddy [24], resulting in a quadratic programming problem. A simple algorithm was suggested in which the programming problem reduced to solution of simultaneous linear equations subject to checks on constraints, violation of which necessitates iteration. In this thesis application of this minimum principle is extended to the incremental analysis of elasto-plastic continua. For the resulting programming problem it is not necessary to piecewise linearize the yield surface as in the formal quadratic programming approach, and thus the continuously differentiable von Mises yield function is assumed. For simplicity. discussion will be limited to two-dimensional problems (plane stress, plane strain and axial symmetry); however the extension to general three-dimensional continua is directly obtained by inclusion of field variable components ignored in the two-dimensional case. 
SOME FUNDAMENTAL CONCEPTS

\section{$2.1 \quad$ Introduction}

In this thesis discussion is limited to bodies composed of an isotropic, homogeneous material. In the plastic range the material is assumed to obey the von Mises yield condition, and be either elastic-perfectly plastic, or linear kinematic hardening. Deformations are assumed to be isothermal and small in the sense that kinematic relations are linear in strain and displacement and equilibrium equations linear in stress and force. Loading of the body is assumed to be quasi-static so that inertia terms can be ignored.

In developing the general relationships governing the deformation of an elasto-plastic continuum, consider a body of volume $V$ and surface $S$ in a Cartesian coordinate system $x_{i}(i=1,2,3)$. The body is subjected to body forces $F_{i}\left(x_{k}\right)$ on $V$, and surface tractions $T_{i}\left(x_{k}\right)$ on part of the surface $S_{T}$. On the remainder of the surface $S_{u}$ displacements $u_{i}\left(x_{k}\right)$ are prescribed. The governing relations comprise equilibrium equations, kinematic relations and constitutive relations.

\section{$2.2 \quad$ Equilibrium}

The equilibrium equations are characterized by

$$
\frac{\partial \sigma_{i j}}{\partial x_{j}}+F_{i}=0 \text { on } V \text {, }
$$




$$
\sigma_{i j}=\sigma_{j i},
$$

and $\quad \sigma_{i j} \nu_{j}=T_{i}$ on $S$,

where $\sigma_{i j}(i, j=1,2,3)$ is the stress tensor, and $v_{i}$ is the outward normal vector at a point on the surface. A statically admissible set of body forces $F_{i}$, surface tractions $T_{i}$, and stresses $\sigma_{i j}$ must satisfy equations (2.1), (2.2) and (2.3).

\subsection{Kinematic Relations}

The strain field $\varepsilon_{i j}$ is obtained from the displacement field $u_{i}$ by means of the strain-displacement relations

$$
\varepsilon_{i j}=\frac{1}{2}\left(\frac{\partial u_{i}}{\partial x_{j}}+\frac{\partial u_{j}}{\partial x_{i}}\right)
$$

Further, the compatibility condition ensures the integrability of the strain field to within a rigid body motion, and may be expressed as

$$
\frac{\partial^{2} \varepsilon_{i j}}{\partial x_{k} \partial x_{l}}+\frac{\partial^{2} \varepsilon_{k \ell}}{\partial x_{i} \partial x_{j}}=\frac{\partial^{2} \varepsilon_{i k}}{\partial x_{j} \partial x_{l}}+\frac{\partial^{2} \varepsilon_{j l}}{\partial x_{i} \partial x_{k}} .
$$

A kinematically admissible set of strains and displacements must satisfy equations (2.4) and (2.5).

\subsection{Constitutive Relations}

The constitutive relations are written after breaking the strain tensor $\varepsilon_{i j}$ into an elastic part $\varepsilon_{i j}^{e}$ and a plastic part $\varepsilon_{i j}^{p}$ such that 


$$
\varepsilon_{i j}=\varepsilon_{i j}^{e}+\varepsilon_{i j}^{p}
$$

The elastic strain and stress are linearly related:

$$
\varepsilon_{i j}^{e}=C_{i j k l} \sigma_{k \ell},
$$

where $C_{i j k l}$ is an isotoropic fourth order tensor.

A yield function $\phi$ is introduced to describe plastic behaviour. In this thesis it is assumed that the yield function is a convex continuously differentiable scalar function and may be written as $\phi=\phi\left(\sigma_{i . j}\right)$ for elastic-perfectly plastic materials. For elastic-plastic or hardening behaviour we shall limit our discussion to a linear kinematic hardening model for the material. In this case the subsequent yield function may be written as $\phi=\phi\left(\sigma_{i j}, \varepsilon_{i j}^{p}\right)$, where the plastic strains $\varepsilon_{i j}^{p}$ represent the history of plastic strain from the virgin unstressed state, [25].

Yielding occurs when $\phi=0$ and stress states such that $\phi>0$ are inadmissible. The plastic strains remain unchanged for any stress increment imposed on a stress state for which $\phi<0$ (elastic behaviour), or for which $\phi=0$ and $\frac{\partial \phi}{\partial \sigma_{i j}} d \sigma_{i j}<0$ (unloading). Thus changes in plastic strains can only occur for stress increments imposed on stress states for which $\phi=0$ (yielding) and $\frac{\partial \phi}{\partial \sigma_{i j}} d \sigma_{i j} \geq 0$ (Ioading or neutral loading).

Figure 2.1 shows directions of stress increments from points on the yield surface $\phi=0$ in stress space. In the case of hardening $\phi=\phi\left(\sigma_{i j)} \varepsilon_{i j}^{p}\right)$ and thus the curve $\phi=0$ in stress subspace represents the current yield surface corresponding to current plastic strains. 


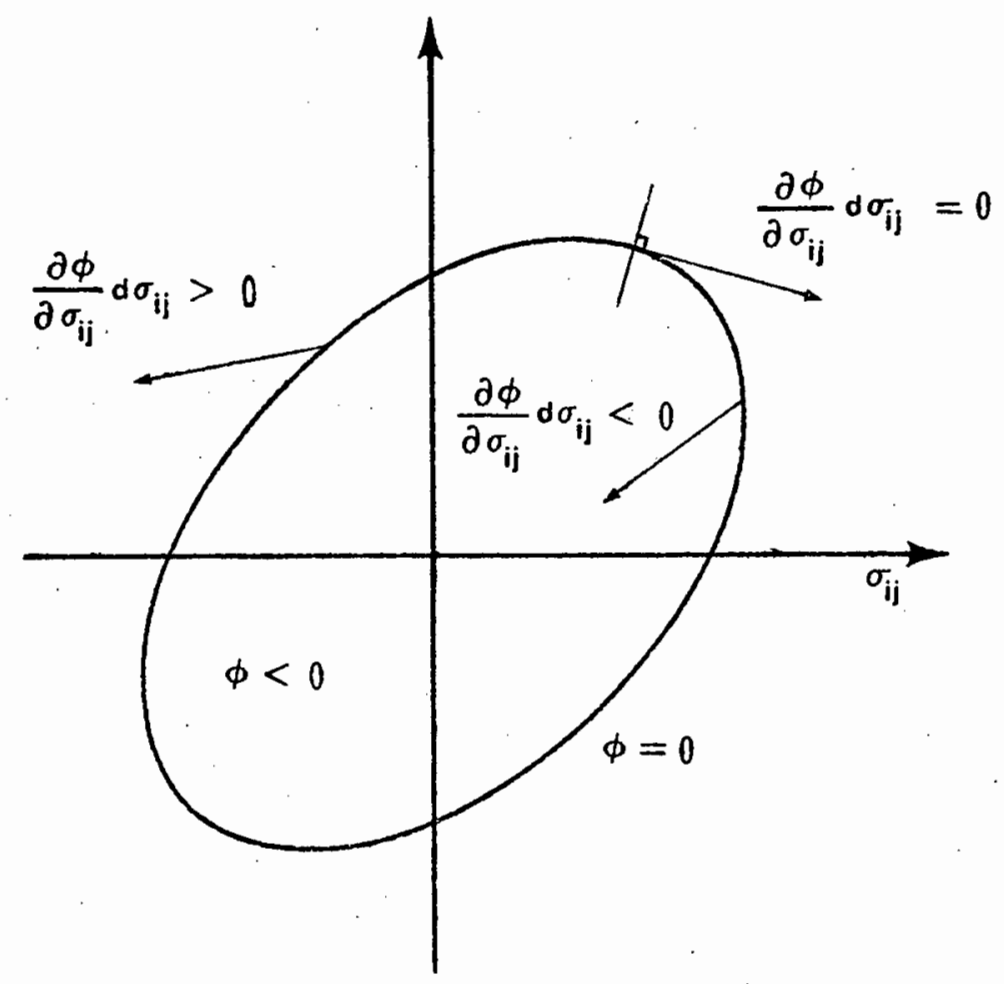

Figure 2.1 Directions of stress increments in stress space

When plastic strain changes do occur the plastic strain increment is proportional to the gradient of the yield function. Defining a non-negative scalar field $\lambda\left(x_{i}\right)$, we write the plastic strain increment as

$$
\begin{aligned}
d \varepsilon_{i j}^{p}=0 \text { if } \phi & <0, \\
& \text { or } \phi=0 \text { and } \frac{\partial \phi}{\partial \sigma_{i j}} d \sigma_{i j}<0 ;
\end{aligned}
$$

and $\quad d \varepsilon_{i j}^{p}=\lambda \frac{\partial \phi}{\partial \sigma_{i j}}$ if $\phi=0$ and $\frac{\partial \phi}{\partial \sigma_{i j}} d \sigma_{i j} \geq 0$. 
In the case of an elastic-plastic or hardening material

$$
\lambda=G \frac{\partial \phi}{\partial \sigma_{k \ell}} d \sigma_{k \ell}
$$

where the scalar $G$ is positive definite. We shall consider the more general problem of a hardening solid as the elastic-perfectly plastic problem can be recovered as a special case.

For perfect plasticity stress changes such that $\frac{\partial \phi}{\partial \sigma_{i j}} d \sigma_{i j}>0$ are inadmissible since they lead to stress states for which $\phi\left(\sigma_{i j}\right)>0$. From equation (2.9) it follows that $\lambda$ can then only be non-zero when $\frac{\partial \phi}{\partial \sigma_{i j}} d \sigma_{i j}=0$, and hence in equation (2.10) $\lambda$ can only be finite and non-zero if $G \rightarrow \infty$. Thus the elastic-perfectly plastic case is recovered from the elastic plastic case as the limit $G \rightarrow \infty$, in which case $\lambda$ is non-negative but otherwise undetermined.

\subsection{Von Mises Yield Condition and Linear Kinematic Hardening}

The von Mises initial yield condition [26] assumes that plastic deformation becomes possible when the shear stress on a particular plane, the octahedral plane which is equally inclined to the three principal axes, reaches a limiting magnitude $\mathrm{k}$. This is conventionally written in quadratic form so that the von Mises initial yield function is

$$
\phi=\sigma_{\text {so }}^{2}-\mathrm{k}^{2}
$$

where $\sigma_{\text {so }}$ is the octahedral shear stress. Expanding $\sigma_{\text {so }}$ in terms of stress components in arbitrary Cartesian coordinate directions this becomes 


$$
\phi=\frac{1}{3}\left\{\sigma_{i j} \sigma_{i j}-\frac{1}{3}\left(\sigma_{k k}\right)^{2}\right\}-k^{2}
$$

For a hardening material the yield function is $\phi=\phi\left(\sigma_{i j}, \varepsilon_{i j}^{p}\right)$. Adopting a kinematic hardening model such that subsequent yield surfaces are translations of the initial yield surface in stress space, retaining a constant shape, size and orientation, we may write the von Mises subsequent yield function as

$$
\phi=\frac{1}{3}\left\{\left(\sigma_{i j}-c \varepsilon_{i j}^{p}\right)\left(\sigma_{i j}-c \varepsilon_{i j}^{p}\right)-\frac{l}{3}\left(\sigma_{k k}-c \varepsilon_{k k}^{p}\right)^{2}\right\}-k^{2},
$$

where $c$ is a constant. (The term $c \varepsilon_{k k}^{p}$ vanishes since there is zero volume change associated with plastic deformation).

The most convenient idealization for the scalar hardening coefficient $G$ in equation (2.10) is

$$
\frac{I}{G}=c\left(\frac{\partial \phi}{\partial \sigma_{k \ell}} \frac{\partial \phi}{\partial \sigma_{k \ell}}\right)
$$

This leads to a bilinear stress-strain curve in a monotonic loading test in simple tension, from which $c=E_{p}$, the plastic modulus, [25]. Thus writing

$$
\bar{\sigma}_{i j}=\left(\sigma_{i, j}-E_{p} \varepsilon_{i j}^{p}\right),
$$

the von Mises yield function becomes

$$
\phi=\frac{1}{3}\left\{\bar{\sigma}_{i j} \bar{\sigma}_{i j}-\frac{1}{3}\left(\bar{\sigma}_{k k}\right)^{2}\right\}-k^{2},
$$


where $\bar{\sigma}_{\mathrm{kk}}=\sigma_{\mathrm{kk}}$. The limiting value of octahedral shear stress $\mathrm{k}$ is conventionally related to the uniaxial yield stress $\sigma_{0}$ as

$$
\mathrm{k}^{2}=\frac{2}{9} \sigma_{0}^{2}
$$

giving finally

$$
\phi=\frac{1}{3}\left\{\bar{\sigma}_{i j} \bar{\sigma}_{i j}-\frac{1}{3}\left(\bar{\sigma}_{k k}\right)^{2}-\frac{2}{3} \sigma_{o}^{2}\right\}
$$

The elastic-perfectly plastic case is recovered by setting $E_{p}=0$, in which case $\bar{\sigma}_{i j}=\sigma_{i j}$.

\section{$2.6 \quad$ Plane Stress}

In formulating particular continuum analysis problems it is often possible to reduce the complexity of a general three-dimensional formulation to one of two dimensions. Such idealizations include problems in plane stress, plane strain and axial symetry. In this thesis we shall limit our discussion to this class of problems, although the formulation presented may be readily extended to general three-dimensional continua.

In the case of plane stress we consider thin sheets (plates) of material subjected to loads and imposed displacements at the boundary of the sheet and in the plane of the sheet. Let such a sheet lie in the $x, y$ plane of a Cartesian coordinate system $x, y, z$. The nonzero stress components are $\sigma_{x x}, \sigma_{y y}$ and $\sigma_{x y}$, which are assumed to be constant through the thickness of the sheet (z-direction), while the components $\sigma_{\mathrm{zz}}, \sigma_{\mathrm{yz}}$ and $\sigma_{\mathrm{zx}}$ are taken to be zero throughout the body. 
In consequence the associated strain components $\varepsilon_{\mathrm{zz}}, \varepsilon_{\mathrm{yz}}$ and $\varepsilon_{\mathrm{zx}}^{\dagger}$ are ignored in the analysis, which leads to minor violations of the compatibility condition (equation 2.5). However, the assumptions of plane stress provide very good approximations for thin plates under in-plane loading.

If $\mathrm{u}$ and $\mathrm{v}$ are respectively the displacement components in the $\mathrm{x}$ and $y$ directions, then the strain-displacement relations (equation 2.4) reduce to

$$
\varepsilon_{x x}=\frac{\partial u}{\partial x}, \varepsilon_{y y}=\frac{\partial v}{\partial y}, \varepsilon_{x y}=\frac{\partial u}{\partial y}+\frac{\partial v}{\partial x}
$$

For an isotropic material the elastic constitutive relations (equation 2.7) are

$$
\begin{aligned}
& \varepsilon_{x x}^{e}=\frac{1}{E}\left(\sigma_{x x}-v \sigma_{y y}\right), \\
& \varepsilon_{y y}^{e}=\frac{1}{E}\left(\sigma_{y y}-v \sigma_{x x}\right),
\end{aligned}
$$

and $\quad \varepsilon_{x y}^{e}=\frac{2(1+v)}{E} \sigma_{x y}$,

where $E$ is the elastic modulus and $v$ is Poisson's ratio. Further, the von Mises yield condition for a kinematic hardening material (equation $2: 18$ ) in plane stress reduces to

$\dagger$

shear strains with Cartesian subscripts $\mathrm{x}, \mathrm{y}, \mathrm{z}$ denote engineering (and not tensorial) shear strains, e.g. $\varepsilon_{x y}=\varepsilon_{12}+\varepsilon_{21}=2 \varepsilon_{12}=2 \varepsilon_{21}$. 


$$
\phi=\frac{2}{9}\left\{\bar{\sigma}_{\mathrm{xx}}^{2}+\bar{\sigma}_{\mathrm{yy}}^{2}-\bar{\sigma}_{\mathrm{xx}} \bar{\sigma}_{\mathrm{yy}}+3 \bar{\sigma}_{\mathrm{xy}}^{2}-\sigma_{0}^{2}\right\}
$$

where $\bar{\sigma}_{\mathrm{xx}}=\sigma_{\mathrm{xx}}-\mathrm{E}_{\mathrm{p}} \varepsilon_{\mathrm{xx}}^{\mathrm{p}}$, etc.

\section{$2.7 \quad$ Plane Strain}

A body is considered to be in a state of plane strain if it extends a large (theoretically infinite) distance in, say, the z-direction, and has boundary conditions independent of $z$. In this case a representative sheet of unit thickness is considered in the analysis, as the displacement components $u, v$ are functions of $x$ and $\mathrm{y}$ only, and displacement in the z-direction is zero. It is evident that the strain components $\varepsilon_{\mathrm{zz}}, \varepsilon_{\mathrm{yz}}$ and $\varepsilon_{\mathrm{zx}}$ are zero.

The stress components $\sigma_{x x}, \sigma_{y y}$ and $\sigma_{x y}$ can be non-zero, but although the shear components $\sigma_{\mathrm{yz}}$ and $\sigma_{\mathrm{zx}}$ are taken to be zero throughout the body, in general $\sigma_{\mathrm{zz}}$ does not vanish. Thus if plastic deformation occurs $(\phi=0)$ then

$$
\mathrm{d} \varepsilon_{\mathrm{zz}}^{\mathrm{p}}=\lambda \frac{\partial \phi}{\partial \sigma_{\mathbf{z z}}}
$$

However, plane strain assumptions give the total strain in the z-direction to be zero. Therefore, from the incremental form of equation (2.6) we have

$$
\begin{aligned}
\mathrm{d} \varepsilon_{\mathrm{zz}} & =\mathrm{d} \varepsilon_{\mathrm{zz}}^{\mathrm{e}}+\mathrm{d} \varepsilon_{\mathrm{zz}}^{\mathrm{p}}=0, \\
\text { or } \quad \mathrm{d} \varepsilon_{\mathrm{zz}}^{\mathrm{e}} & =-\mathrm{d} \varepsilon_{\mathrm{zz}}^{\mathrm{p}},
\end{aligned}
$$

a condition which must be imposed in the analysis. 
Thus the strain-displacement relations (equation 2.4) reduce to those of the plane stress problem (equation 2.19) with the additional relation $\varepsilon_{\mathrm{zz}}=0$. For an isotropic material the elastic constitutive relations of equation (2.7) become

$$
\begin{aligned}
& \varepsilon_{x x}^{e}=\frac{1}{E}\left\{\sigma_{x x}-v\left(\sigma_{y y}+\sigma_{z z}\right)\right\}, \\
& \varepsilon_{y y}^{e}=\frac{1}{E}\left\{\sigma_{y y}-v\left(\sigma_{x x}+\sigma_{z z}\right)\right\}, \\
& \varepsilon_{z z}^{e}=\frac{1}{E}\left\{\sigma_{z z}-v\left(\sigma_{x x}+\sigma_{y y}\right)\right\},
\end{aligned}
$$

and $\varepsilon_{x y}^{e}=\frac{2(1+v)}{E} \sigma_{x y}$.

The von Mises yield function for a body in plane strain and composed of a linear kinematic hardening material is

$$
\phi=\frac{2}{9}\left\{\bar{\sigma}_{\mathrm{xx}}^{2}+\bar{\sigma}_{\mathrm{yy}}^{2}+\bar{\sigma}_{\mathrm{zz}}^{2}-\bar{\sigma}_{\mathrm{xx}} \bar{\sigma}_{\mathrm{yy}}-\bar{\sigma}_{\mathrm{yy}} \bar{\sigma}_{\mathrm{zz}}-\bar{\sigma}_{\mathrm{zz}} \bar{\sigma}_{\mathrm{xx}}+3 \bar{\sigma}_{\mathrm{xy}}^{2}-\sigma_{0}^{2}\right\} .
$$

\subsection{Axial Symmetry}

A problem frequently encountered in the analysis of continua is that of a body of revolution (axisymmetric solid) under axisymmetric loading. As in the cases of plane stress and plane strain the geometric representation can be reduced to one of two dimensions. From considerations of symmetry the state of strain at a point in the body is completely described by two displacement components lying in the plane containing the point and the axis of symmetry. 
A polar coordinate system is conventionally employed. Let $r$ and $z$ denote respectively the radial and axial directions, and let $\theta$ denote the circumferential or tangential coordinate direction. Any displacement in the radial direction will cause a circumferential strain $\varepsilon_{\theta \theta}$, but since stresses and strains are symmetrical with respect to the $\mathrm{z}$-axis and are therefore independent of $\theta$, it follows that the stress components $\sigma_{r \theta}, \sigma_{z \theta}$ and strain components $\varepsilon_{r \theta}, \varepsilon_{z \theta}$ must vanish throughout the body.

If $u$ and $v$ are respectively the displacement components in the $r$ and $z$ directions then the strain-displacement relations may be written as [27]

$$
\varepsilon_{r r}=\frac{\partial u}{\partial r}, \varepsilon_{z z}=\frac{\partial v}{\partial z}, \varepsilon_{\theta \theta}=\frac{\underline{u}}{r}, \varepsilon_{r z}=\frac{\partial u}{\partial z}+\frac{\partial v}{\partial r}
$$

For an isotropic material the elastic constitutive relations are

$$
\begin{aligned}
& \varepsilon_{r r}^{e}=\frac{1}{E}\left\{\sigma_{r r}-v\left(\sigma_{z z}+\sigma_{\theta \theta}\right)\right\}, \\
& \varepsilon_{z z}^{e}=\frac{1}{E}\left\{\sigma_{z z}-v\left(\sigma_{r r}+\sigma_{\theta \theta}\right)\right\}, \\
& \varepsilon_{\theta \theta}^{e}=\frac{1}{E}\left\{\sigma_{\theta \theta}-v\left(\sigma_{r r}+\sigma_{z z}\right)\right\},
\end{aligned}
$$

and $\quad \varepsilon_{r z}^{e}=\frac{2(I+v)}{E} \sigma_{r z}$.

For the axisymmetric case the von Mises yield function of equation (2.18) reduces to 


$$
\phi=\frac{2}{9}\left\{\bar{\sigma}_{r r}^{2}+\bar{\sigma}_{z z}^{2}+\bar{\sigma}_{\theta \theta}^{2}-\bar{\sigma}_{r r} \bar{\sigma}_{z z}-\bar{\sigma}_{z z} \bar{\sigma}_{\theta \theta}-\bar{\sigma}_{\theta \theta} \bar{\sigma}_{r r}+3 \bar{\sigma}_{r z}^{2}-\sigma_{0}^{2}\right\} .
$$


C H A P T E R 3

THE KINEMATIC MINIMUM PRINCIPLE FOR THE

RATE PROBLEM IN ELASTO-PLASTICITY

\subsection{Introduction}

As a fundamental problem in elasto-plasticity we consider the response of a body to successive increments of load throughout its entire.stress history. This incremental analysis is formulated initially in terms of rates (time derivatives) of the field variables.

\subsection{The Classical Rate Problem in Elasto-Plasticity}

The classical rate problem in elasto-plasticity may be stated as follows. Consider a body of volume $V$ and surface $S$ in a Cartesian coordinate system $x_{i}$, subjected to known body force rates $\dot{F}_{i}\left(x_{j}\right)$ on $V$, known traction rates $\dot{\mathrm{T}}_{i}\left(\mathrm{x}_{j}\right)$ on part of the surface $\mathrm{S}_{\mathrm{T}}$, and known displacement rates $\dot{u}_{i}\left(x_{j}\right)$ on the remainder of the surface $S_{u}$. As solution to the rate problem we seek displacement rates $\dot{u}_{i}\left(x_{j}\right)$ on $S_{T}$ and $V$, reaction rates $\dot{T}_{i}\left(x_{j}\right)$ on $S_{u}$, a stress rate field $\dot{\sigma}_{i j}\left(x_{k}\right)$, and a strain rate field $\dot{\varepsilon}_{i j}\left(x_{k}\right)$. The governing equations comprise the rate forms for infinitesimal displacement of the equilibrium equations, kinematic relations and constitutive relations.

Since the equilibrium equations and kinematic relations (equation 2.1 through 2.5) are linear in force, stress, displacement and strain, it follows that the rate forms of these equations will be 
linear in force rates, stress rates, displacement rates and strain rates, and thus

$$
\begin{aligned}
& \frac{\partial \dot{\sigma}_{i j}}{\partial x_{j}}+\dot{F}_{i}=0 \text { on } v, \\
& \dot{\sigma}_{i j} v_{j}=\dot{T}_{i} \text { on } s,
\end{aligned}
$$

and $\quad \dot{\varepsilon}_{i j}=\frac{1}{2}\left(\frac{\partial \dot{u}_{i}}{\partial x_{j}}+\frac{\partial \dot{u}_{j}}{\partial x_{i}}\right)$ on $v$.

Rewriting the constitutive equations of section 2.4 in rate form we have

$$
\begin{aligned}
& \dot{\varepsilon}_{i j}=\dot{\varepsilon}_{i j}^{e}+\dot{\varepsilon}_{i j}^{p}, \\
& \dot{\varepsilon}_{i j}^{e}=c_{i j k l} \dot{\sigma}_{k l}, \\
& \dot{\varepsilon}_{i j}^{p}=0 \quad \text { if } \phi<0 \\
& \quad \text { or } \phi=0 \text { and } \frac{\partial \phi}{\partial \sigma_{i j}} \dot{\sigma}_{i j}<0, \\
& \dot{\varepsilon}_{i j}^{p}=\lambda \frac{\partial \phi}{\partial \sigma_{i j}} \quad \text { if } \phi=0 \text { and } \frac{\partial \phi}{\partial \sigma_{i j}} \dot{\sigma}_{i j} \geq 0
\end{aligned}
$$

and where for hardening materials

$$
\lambda=\mathrm{G} \frac{\partial \phi}{\partial \sigma_{\mathrm{k} \ell}} \dot{\sigma}_{\mathrm{k} \ell}
$$

with $G$ a positive definite scalar. For elastic-perfectly plastic behaviour $\lambda$ is non-negative but otherwise undefined. 
Although all the governing equations (3.1 through 3.8) are linear in rates of the field variables, it is not known a priori whether loading, neutral loading or unloading will occur in plastic regions of the body $(\phi=0)$, and thus the rate problem is not truly linear.

The rate problem at time $t$ may be considered to be preceded by a succession of rate problems over the time interval $0 \leq \tau \leq t$. The response of the body over this interval is characterized by body forces $F_{i}\left(x_{j}, \tau\right)$, surface tractions $T_{i}\left(x_{j}, \tau\right)$, displacements $u_{i}\left(x_{k}, \tau\right)$, stresses $\sigma_{i j}\left(x_{k}, \tau\right)$ and strains $\varepsilon_{i j}\left(x_{k}, \tau\right)$. It is assumed that at time $t=0$ the body is unstressed and the material in its virgin state.

Knowing the complete solution at time $t$ we consider body force rates $\dot{\mathrm{F}}_{i}$ on $\mathrm{V}$, surface traction rates $\dot{\mathrm{T}}_{i}$ on $\mathrm{S}_{\mathrm{T}}$, and displacement rates $\dot{u}_{i}$ on $\mathrm{S}_{\mathrm{u}}$. The rate forms of the governing equations permit a unique solution for the traction rates $\dot{\mathrm{T}}_{i}$ on $\mathrm{S}_{u}$, displacement rates $\dot{\mathrm{u}}_{i}$ on $S_{T}$ and $V$, the stress rate field $\dot{\sigma}_{i j}$ and the strain rate field $\dot{\varepsilon}_{i j}$. Before formulating the kinematic minimum principle for the solution of the rate problem, it is necessary to discuss inversion of the constitutive relations to give the stress rate $\dot{\sigma}_{i j}$ in terms of the total strain rate $\dot{\varepsilon}_{i j}$.

\subsection{Inversion of Constitutive Equations}

Denoting the inverse of $C_{i j k l}$ by $D_{i j k l}$, equation (3.5) is inverted as

$$
\dot{\sigma}_{i j}=D_{i j k \ell} \dot{\varepsilon}_{k \ell}^{e} \text {. }
$$


Hence, for $\dot{\varepsilon}_{i j}^{p}=0$ we have

$$
\begin{aligned}
\dot{\sigma}_{i j}=D_{i j k l} \varepsilon_{k l} & \text { for } \phi<0 \\
\text { or } \phi & =0 \text { and } D_{i j k l} \frac{\partial \phi}{\partial \sigma_{i j}} \dot{\varepsilon}_{k l} \leq 0 .
\end{aligned}
$$

If for hardening behaviour $\dot{\varepsilon}_{i j}^{p} \neq 0$, then substituting for the elastic and plastic strain rates, equations $(3.5),(3.7)$ and $(3.8)$, in the expression for the total strain rate, equation (3.4), we have

$$
\dot{\varepsilon}_{i j}=C_{i j k l} \dot{\sigma}_{k l}+G \frac{\partial \phi}{\partial \sigma_{i j}} \frac{\partial \phi}{\partial \sigma_{k l}} \dot{\sigma}_{k l} .
$$

Rearranging as

$$
C_{i j k \ell} \dot{\sigma}_{k \ell}=\dot{\varepsilon}_{i j}-G \frac{\partial \phi}{\partial \sigma_{i j}} \frac{\partial \phi}{\partial \sigma_{k \ell}} \dot{\sigma}_{k \ell}
$$

and premultiplying by $D_{i j k l}$. gives

$$
\dot{\sigma}_{i j}=D_{i j k \ell}\left(\dot{\varepsilon}_{k \ell}-G \frac{\partial \phi}{\partial \sigma_{k \ell}} \frac{\partial \phi}{\partial \sigma_{m n}} \dot{\sigma}_{m n}\right) \text {. }
$$

Further, multiplying by $\frac{\partial \phi}{\partial \sigma_{i j}}$ leads to

$$
\frac{\partial \phi}{\partial \sigma_{i j}} \dot{\sigma}_{i j}=D_{i j k \ell} \frac{\partial \phi}{\partial \sigma_{i j}} \dot{\varepsilon}_{k \ell}-G D_{m n p q} \frac{\partial \phi}{\partial \sigma_{m n}} \frac{\partial \phi}{\partial \sigma_{p q}} \frac{\partial \phi}{\partial \sigma_{i j}} \dot{\sigma}_{i j}
$$




$$
\frac{\partial \phi}{\partial \sigma_{i j}} \dot{\sigma}_{i j}=\frac{D_{i j k \ell} \frac{\partial \phi}{\partial \sigma_{i j}} \dot{\varepsilon}_{k \ell}}{\left(1+G D_{m n p q} \frac{\partial \phi}{\partial \sigma_{m n}} \frac{\partial \phi}{\left.\partial \sigma_{p q}\right)}\right.}
$$

Substituting this expression in equation (3.13) and multiplying out gives finally

$$
\cdot \dot{\sigma}_{i j}=D_{i j k l} \dot{\varepsilon}_{k \ell}-D_{i j k l} \frac{\partial \phi}{\partial \sigma_{k \ell}}\left[\frac{D_{p q r s} \frac{\partial \phi}{\partial \sigma_{p q}} \dot{\varepsilon}_{r s}}{\frac{1}{G}+D_{m n g} \frac{\partial \phi}{\partial \sigma_{m n}} \frac{\partial \phi}{\partial \sigma_{h g}}}\right]
$$

for $\phi=0$ and $D_{i j k \ell} \frac{\partial \phi}{\partial \sigma_{i j}} \dot{\varepsilon}_{k \ell} \geq 0$

Equations (3.10) and (3.15) are thus the inverted constitutive rate equations for hardening behaviour, the elastic-perfectly plastic case being recovered as the limit $G \rightarrow \infty$ in equation (3.15). The inversion of the constitutive equations may be shown to be unique, [25].

\subsection{The Kinematic Minimum Principle for the Rate Problem}

Martin [23] has proposed an extended kinematic minimum principle for the rate problem in elasto-plasticity. Consider the inverted constitutive equations $(3.10)$ and (3.15). These may be derived from a discontinuous potential functional $\mathrm{w}^{\circ}$ defined by

$$
W^{\circ}=W^{\circ}\left(\dot{\varepsilon}_{i j}\right), \quad \dot{\sigma}_{i j}=\frac{\partial W^{\circ}}{\partial \varepsilon_{i j}} .
$$


where

$$
W^{O}=\frac{1}{2} D_{i j k l} \dot{\varepsilon}_{i j} \dot{\varepsilon}_{k \ell}
$$

for $\phi<0$

$$
\text { or } \phi=0 \text { and } D_{i j k \ell} \frac{\partial \phi}{\partial \sigma_{i j}} \dot{\varepsilon}_{k \ell} \leq 0
$$

and

$$
W^{\circ}=\frac{1}{2} D_{i j k l} \dot{\varepsilon}_{i j} \dot{\varepsilon}_{k l}-\frac{1}{2} \frac{\left(D_{i j k l} \frac{\partial \phi}{\partial \sigma_{i j}} \dot{\varepsilon}_{k l}\right)^{2}}{\left(\frac{1}{G}+D_{p q r s} \frac{\partial \phi}{\partial \sigma_{p q}} \frac{\partial \phi}{\partial \sigma_{r s}}\right)}
$$

for $\phi=0$ and $D_{i j k l} \frac{\partial \phi}{\partial \sigma_{i j}} \dot{\varepsilon}_{k \ell} \geq 0$.

So as to construct a kinematic minimum principle for the rate problem let us suppose that $\dot{\varepsilon}_{i j}^{*}, \dot{u}_{i}^{*}$ defined on $V$ satisfy the rate form of the strain-displacement relations (equation 3.3) and the kinematic boundary conditions $\dot{u}_{i}^{*}=\dot{u}_{i}$ on $S_{u}$. The solution of the rate problem is that member of the class $\dot{\varepsilon}_{i j}^{*}$, $\dot{u}_{i}^{*}$ which renders an absolute minimum the functional

$$
U_{p}^{\circ}\left(\dot{\varepsilon}_{i j}^{*}, \dot{u}_{i}^{*}\right)=\int_{V} W^{\circ}\left(\dot{\varepsilon}_{i j}^{*}\right) d V-\int_{V} \dot{F}_{i} \dot{u}_{i}^{*} d V-\int_{S_{T}} \dot{T}_{i} \dot{u}_{i}^{*} d S
$$

Because the functional $\mathrm{w}^{\circ}$ is discontinuous, Martin broadens the class of variables and replaces $\mathrm{w}^{\circ}$ with a continuous potential functional $\overrightarrow{\mathrm{W}}^{\mathrm{O}}$ subject to inequality constraints. Dividing the body 
into two regions, let the plastic part of the body (where $\phi=0$ ) be denoted collectively by $v_{p}$, and the elastic part of the body (where $\phi<0$ ) be denoted collectively by $\mathrm{V}_{e}$. Defining a nonnegative scalar field $\lambda^{*}\left(x_{k}\right)$ over $v_{p}$, Martin introduces the functional

$$
\begin{aligned}
\widetilde{W}^{O}\left(\dot{\varepsilon}_{i j}^{*}, \lambda^{*}\right)= & \frac{1}{2} D_{i j k \ell}\left(\dot{\varepsilon}_{i j}^{*}-\lambda^{*} \frac{\partial \phi}{\partial \sigma_{i j}}\right)\left(\dot{\varepsilon}_{k \ell}^{*}-\lambda^{*} \frac{\partial \phi}{\partial \sigma_{k \ell}}\right) \\
& +\frac{\left(\lambda^{*}\right)^{2}}{2 G}
\end{aligned}
$$

and the constraints $\lambda^{*}=0$ in $\mathrm{V}_{\mathrm{e}}$

$$
\lambda^{*} \geq 0 \text { in } \mathrm{v}_{\mathrm{p}}
$$

For an arbitrary choice of $\lambda^{*}$

$$
\bar{W}^{\circ}\left(\dot{\varepsilon}_{i j}^{*}, \lambda^{*}\right) \geq W^{O}\left(\dot{\varepsilon}_{i j}\right)
$$

with equality occurring when $\lambda^{*}$ takes the value which gives the minimum value of $\overline{\mathrm{W}}^{\mathrm{O}}$ subject to the constraints of equations (3.21). In this case the actual plastic strain rate is given by

$$
\dot{\varepsilon}_{i j}^{p}=\lambda^{*} \frac{\partial \phi}{\partial \sigma_{i j}}
$$

The proof may be obtained by differentiating the quadratic $\bar{W}^{\circ}$ with respect to $\lambda^{*}$. A stationary value occurs for 


$$
\lambda^{*}=\frac{D_{i j k l} \frac{\partial \phi}{\partial \sigma_{i j}} \dot{\varepsilon}_{k \ell}^{*}}{\frac{1}{G}+D_{p q r s} \frac{\partial \phi}{\partial \sigma_{p q}} \frac{\partial \phi}{\partial \sigma_{r s}}} .
$$

The second derivative of $\overline{\mathrm{W}}^{0}$ with respect to $\lambda^{*}$ is

$$
\frac{I}{G}+D_{i j k \ell} \frac{\partial \phi}{\partial \sigma_{i j}} \frac{\partial \phi}{\partial \sigma_{k \ell}}
$$

and since $G \geq 0$ this expression is positive definite and indicates the stationary point is a minimum.

From equation (3.24) we see that the sign of $\lambda^{*}$ is governed by the sign of $D_{i j k l} \frac{\partial \phi}{\partial \sigma_{i j}} \dot{\varepsilon}_{k \ell}$. Substituting the expression for $\lambda^{*}$ in equation (3.20), the least value of $\bar{W}^{0}$ is given by equation (3.18) if

$$
D_{i j k l} \frac{\partial \phi}{\partial \sigma_{i j}} \dot{\varepsilon}_{k \ell} \geq 0 .
$$

If the expression (3.25) is less than zero, the least value of $\overrightarrow{\mathrm{W}}^{0}$ is given by $\lambda^{*}=0$, in which case $\overline{\mathrm{W}}^{\circ}$ reduces to equation (3.17).

The minimum principle (3.19) is thus extended: the solution of the rate problem is given by that member of the class $\dot{u}^{*}\left(x_{k}\right)$, $\dot{\varepsilon}_{i j}^{*}\left(x_{k}\right), \lambda^{*}\left(x_{k}\right)$ which renders an absolute minimum the functional

$$
\overline{\mathrm{U}}_{\mathrm{p}}^{\mathrm{O}}\left(\dot{\mathrm{u}}_{\dot{i}}^{*}, \dot{\varepsilon}_{\dot{i} j}^{*}, \lambda^{*}\right)=\int_{V} \overline{\mathrm{W}}^{\mathrm{O}}\left(\dot{\varepsilon}_{\dot{\mathrm{j}} \mathrm{j}}^{*}, \lambda^{*}\right) \mathrm{dV}-\int_{\mathrm{V}} \dot{\mathrm{F}}_{i} \dot{\mathrm{u}}_{\dot{i}}^{*} \mathrm{dV}-\int_{S_{\mathrm{T}}} \dot{\mathrm{T}}_{i} \dot{\mathrm{u}}_{\dot{i}}^{*} \mathrm{dS}
$$

subject to the constraints $\lambda^{*}=0$ in $\mathrm{V}_{\mathrm{e}}$ (where $\phi<0$ )

$$
\text { and } \lambda^{*} \geq 0 \text { in } \mathrm{v}_{\mathrm{p}}(\text { where } \phi=0) \text {. }
$$




\section{APPLICATION OF THE KINEMATIC NINIMUM PRINCIPLE \\ TO THE CONTINUUM PROBLEM}

\subsection{Introduction}

The complexity of the governing equations for the extended kinematic minimum principle excludes the possibility of an analytical solution to any realistic problem. However, by employing numerical analysis techniques the minimum principle can be exploited directly. To this end we discretize the spatial field into an assemblage of finite elements. The degree of accuracy with which the numerical solution corresponding to the assemblage of elements approximates the true solution of the original continuum problem, depends on the fineness of the finite element subdivision and the sophistication or complexity of the individual elements.

Limiting discussion to the two-dimensional case we initially consider subdivision of the body into simple triangular constant strain finite elements, as the constant strain (and therefore constant stress) condition within each element enforces elastic-plastic interfaces to occur only at inter-element boundaries. Later the formulation will be extended to higher-order finite elements where numerical integration techniques permit elastic-plastic interfaces to occur within individual elements. 


\subsection{Application to Constant Strain Finite Elements}

We discretize a continuum into an assemblage of $\mathrm{m}$ triangular constant strain finite elements. These give continuity of velocity along common boundaries of adjacent elements. Velocities of the assemblage are described by velocity components at each node.

Consider a generic element lying in the $x-y$ plane of an $x, y, z$ coordinate system, with apices 1,2,3 numbered anti-clockwise and having coordinates $\left(x_{1}, y_{1}\right),\left(x_{2}, y_{2}\right),\left(x_{3}, y_{3}\right)$. Since discussion is limited to problems in plane stress, plane strain or axial symmetry, the velocity field of the element is described by two components of velocity $\dot{u}, \dot{v}$ respectively in the $x, y$ directions, at each apex. Adopting the approach of Zienkiewicz [34], we choose a linear velocity function over the element

$$
\begin{aligned}
& \dot{u}(x, y)=\alpha_{1} x+\alpha_{2} y+\alpha_{3}, \\
& \dot{v}(x, y)=\alpha_{4} x+\alpha_{5} y+\alpha_{6} .
\end{aligned}
$$

The six constants $\alpha_{1}, \ldots, \alpha_{6}$ are obtained by solving six simultaneous equations produced by inserting nodal coordinates and corresponding velocities.

Thus

$$
\begin{aligned}
& \dot{u}(x, y)=\frac{1}{2 \Delta}\left\{x_{1} \dot{u}_{1}+x_{2} \dot{u}_{2}+x_{3} \dot{u}_{3}\right\}, \\
& \dot{v}(x, y)=\frac{1}{2 \Delta}\left\{x_{1} \dot{v}_{1}+x_{2} \dot{v}_{2}+x_{3} \dot{v}_{3}\right\},
\end{aligned}
$$

$$
\text { where } \begin{aligned}
x_{1} & =a_{1}+b_{1} x+c_{1} y \\
x_{2} & =a_{2}+b_{2} x+c_{2} y, \\
x_{3} & =a_{3}+b_{3} x+c_{3} y
\end{aligned}
$$


and where

$$
\begin{aligned}
& a_{1}=x_{2} y_{3}-x_{3} y_{2}, \\
& b_{1}=y_{2}-y_{3}, \\
& c_{1}=x_{3}-x_{2},
\end{aligned}
$$

with remaining coefficients obtained by cyclic permutation of subscripts in the order 1,2,3. Further

$$
2 \Delta=\left|\begin{array}{ccc}
1 & x_{1} & y_{1} \\
1 & x_{2} & y_{2} \\
1 & x_{3} & y_{3}
\end{array}\right|=2 x \text { area of triangle } 1,2,3 .
$$

For the case of plane stress the strain-displacement relations of equations (2.19) give in rate form

$$
\left\{\begin{array}{l}
\dot{\varepsilon}_{\mathrm{xx}} \\
\dot{\varepsilon}_{\mathrm{yy}}
\end{array}\right\}=\frac{1}{2 \Delta}\left[\begin{array}{cccccc}
\mathrm{b}_{1} & 0 & \mathrm{~b}_{2} & 0 & \mathrm{~b}_{3} & 0 \\
0 & c_{1} & 0 & c_{2} & 0 & c_{3} \\
c_{1} & b_{1} & c_{2} & b_{2} & c_{3} & b_{3}
\end{array}\right]\left\{\begin{array}{c}
\dot{u}_{1} \\
\dot{v}_{1} \\
\dot{u}_{2} \\
\dot{v}_{2} \\
\dot{u}_{3} \\
\dot{v}_{3}
\end{array}\right\} \text {, }
$$

and for plane strain 


$$
\left\{\begin{array}{l}
\dot{\varepsilon}_{x x} \\
\dot{\varepsilon}_{y y} \\
\dot{\varepsilon}_{z z} \\
\varepsilon_{x y}
\end{array}\right\}=\frac{1}{2 \Delta}\left[\begin{array}{cccccc}
b_{1} & 0 & b_{2} & 0 & b_{3} & 0 \\
0 & c_{1} & 0 & c_{2} & 0 & c_{3} \\
0 & 0 & 0 & 0 & 0 & 0 \\
c_{1} & b_{1} & c_{2} & b_{2} & c_{3} & b_{3}
\end{array}\right]\left\{\begin{array}{c}
\dot{u}_{1} \\
\dot{v}_{1} \\
\dot{u}_{2} \\
\dot{v}_{2} \\
\dot{u}_{3} \\
\dot{v}_{3}
\end{array}\right\} .
$$

Writing equations $(4.6)$ and (4.7) in matrix form

$$
\left\{\dot{\varepsilon}^{\prime}\right\}=\left[B^{\prime}\right]\left\{\dot{u}^{\prime}\right\}
$$

where the prime denotes an element matrix or vector. Components of $\left\{\dot{u}^{\prime}\right\}$ are element node velocities, and $\left[B^{\prime}\right]$ is a linear matrix which depends on element nodal coordinates only. The third row of [ $\left.\mathrm{B}^{\prime}\right]$ is zero in equation (4.7) enforcing the plane strain requirement of zero total strain in the z-direction.

So as to employ a consistant notation, for the axisymmetric case we replace $\mathrm{r}, \mathrm{z}$ and $\theta$ with coordinate subscripts $\mathrm{x}, \mathrm{y}$ and $\mathrm{z}$ respectively, to give the rate form of the strain-displacement relations (2.26) as 


$$
\left\{\begin{array}{l}
\dot{\varepsilon}_{\mathrm{xx}} \\
\dot{\varepsilon}_{\mathrm{yy}} \\
\dot{\varepsilon}_{\mathrm{zz}} \\
\dot{\varepsilon}_{\mathrm{xy}}
\end{array}\right\}=\frac{1}{2 \Delta}\left[\begin{array}{cccccc}
\mathrm{b}_{1} & 0 & \mathrm{~b}_{2} & 0 & \mathrm{~b}_{3} & 0 \\
0 & c_{1} & 0 & c_{2} & 0 & c_{3} \\
\mathrm{a}_{1} & 0 & \mathrm{~d}_{2} & 0 & \mathrm{~d}_{3} & 0 \\
c_{1} & \mathrm{~b}_{1} & c_{2} & b_{2} & c_{3} & b_{3}
\end{array}\right]
$$

where $d_{1}=\frac{a_{1}}{x}+b_{1}+c_{1} \frac{y}{x}$,

with similar expressions for $d_{2}$ and $d_{3}$ obtained by interchange of subscripts.

In view of equation (4.10) coefficients corresponding to the circumferential strain rate $\dot{\varepsilon}_{\mathrm{zz}}$ are dependent on position within the element. To facilitate integration of the strain rate field by enforcing constant strain conditions, it is convenient to treat the centroidal value of circumferential strain rate as constant across the element. Thus the $x, y$ variables in equation (4.10) are replaced by centroidal values $\bar{x}, \bar{y}$, where

$$
\bar{x}=\frac{1}{3}\left(x_{1}+x_{2}+x_{3}\right), \quad \bar{y}=\frac{1}{3}\left(y_{1}+y_{2}+y_{3}\right) .
$$

Hence equation (4.9) may also be written as $\left\{\dot{\varepsilon}^{\prime}\right\}=\left[B^{\prime}\right]\left\{\dot{u}^{\prime}\right\}$, where $\left[\mathrm{B}^{\prime}\right]$ is a linear matrix depending only on element geometry. Breaking the strain rate tensor into elastic and plastic parts as before, it is convenient to introduce weighted strain rates defined by 


$$
\begin{aligned}
& \dot{\delta}_{i j}^{e}=V^{\prime} \dot{\varepsilon}_{i j}^{e}, \\
& \dot{\delta}_{i j}^{p}=V^{\prime} \dot{\varepsilon}_{i j}^{p}, \\
& \dot{\delta}_{i j}=\dot{\delta}_{i j}^{e}+\dot{\delta}_{i j}^{p},
\end{aligned}
$$

where $V^{\prime}$ is the element volume. In the case of plane stress the element is assumed to be of uniform thickness and in plane strain unit thickness, so that the element volume is given by the product of element area and thickness. For the axisymmetric case the element volume is that of a body of revolution and is therefore given by $2 \pi \bar{x} \Delta$, where $\bar{x}$ is the radius to the element centroid and $\Delta$ is the triangular sectional area. Consider now the assemblage of elements. It is assumed that certain nodal velocity components are constrained to be zero throughout the loading history. Ordering remaining velocity components we define the velocity vector $\{\dot{u}\}$ of say, $n$ components. Ordering the elements of the assemblage and taking weighted strain rate components in turn gives a weighted strain rate vector $\{\dot{\delta}\}$ of say, k components. Using the strain rate-velocity relationship of equation (4.8) for each element of the assemblage and taking account of the element volume leads to $a k$ by $n$ weighted deformation matrix [B] for the system:

$$
\{\dot{\delta}\}=[B]\{\dot{u}\} .
$$

The inverted elastic constitutive equations for an isotropic material are written in matrix form for an element as

$$
\left\{\sigma^{\prime}\right\}=\left[D^{\prime}\right]\left\{\varepsilon^{e}\right\},
$$


where for plane stress

$$
\left[D^{\prime}\right]=\frac{E}{1-v^{2}} \cdot\left[\begin{array}{ccc}
1 & v & 0 \\
v & 1 & 0 \\
0 & 0 & \frac{1-v}{2}
\end{array}\right] \text {, }
$$

and for plane strain or axial symmetry

$$
\left[D^{\prime}\right]=\frac{E(1-\nu)}{(1+\nu)(1-2 v)}\left[\begin{array}{cccc}
1 & \frac{\nu}{1-\nu} & \frac{\nu}{1-\nu} & 0 \\
\frac{\nu}{1-\nu} & 1 & \frac{\nu}{1-\nu} & 0 \\
\frac{\nu}{1-\nu} & \frac{\nu}{1-\nu} & 1 & 0 \\
0 & 0 & 0 & \frac{1-2 \nu}{2(1-\nu)}
\end{array}\right] \text {. }
$$

$(4.15 b)$

Writing the constitutive relations (4.14) in terms of weighted strains and in rate form gives

$$
\left\{\dot{\sigma}^{\prime}\right\}=\frac{1}{V^{\prime}}\left[D^{\prime}\right]\left\{\delta^{e}\right\}^{\prime} .
$$

Taking stress rate components of the ordered elements in the same manner as the weighted strain rate vector $\{\dot{\delta}\}$ we define a stress rate vector $\{\dot{0}\}$, also of $\mathrm{k}$ components. Using equation (4.16) for each of the 
ordered elements in turn, we may assemble a weighted elasticity matrix [D] such that

$$
\{\dot{\sigma}\}=[D]\left\{\dot{\delta}^{e}\right\} \text {. }
$$

The matrix [D] is $\mathrm{k}$ by $\mathrm{k}$, symmetric and block-diagonal.

Weighted plastic strain rates are given by

$$
\delta_{i j}^{p}=v \cdot \lambda \frac{\partial \phi}{\partial \sigma_{i j}}
$$

Differentiating the von Mises yield function for plane stress (equation 2.21) gives

$$
\left\{\begin{array}{c}
\dot{\delta}_{x x}^{p} \\
\delta_{y y}^{p} \\
\delta_{x y}^{p}
\end{array}\right\}=v \cdot \lambda\left\{\begin{array}{c}
2 \bar{\sigma}_{x x}-\bar{\sigma}_{y y} \\
2 \bar{\sigma}_{y y}-\bar{\sigma}_{x x} \\
6 \bar{\sigma}_{x y}
\end{array}\right\},
$$

where $\bar{\sigma}_{\mathrm{xx}}=\sigma_{\mathrm{xx}}-E_{\mathrm{p} x \mathrm{xx}} \varepsilon^{\mathrm{p}}$, etc.

For plane strain or axial symmetry the yield functions of equations (2.25) or (2.28) give plastic strain rates as 


$$
\left\{\begin{array}{l}
\dot{\delta}_{x x}^{p} \\
\delta_{y y}^{p} \\
\dot{\delta}_{z z}^{p} \\
\dot{\delta}_{x y}^{p}
\end{array}\right\}=v^{\prime} \lambda\left\{\begin{array}{c}
2 \bar{\sigma}_{x x}-\bar{\sigma}_{y y}-\bar{\sigma}_{z z} \\
2 \bar{\sigma}_{y y}-\bar{\sigma}_{x x}-\bar{\sigma}_{z z} \\
2 \bar{\sigma}_{z z}-\bar{\sigma}_{x x}-\bar{\sigma}_{y y} \\
6 \bar{\sigma}_{x y}
\end{array}\right\} \text {. }
$$

It is convenient to normalize the gradient vector $\frac{\partial \phi}{\partial \sigma_{i j}}$ by setting

$$
\left\{\frac{\partial \phi}{\partial \sigma_{i j}}\right\}^{\prime}=\frac{\partial \phi}{\partial \sigma_{i j}}\left(\frac{\partial \phi}{\partial \sigma_{k \ell}} \frac{\partial \phi}{\partial \sigma_{k \ell}}\right)^{-\frac{1}{2}},
$$

and introduce a different scalar $\Lambda$ such that the weighted plastic strain rates of equation (4.18) become

$$
\dot{\delta}_{i j}^{p}=\Lambda\left\{\frac{\partial \phi}{\partial \sigma_{i j}}\right\}^{\prime}
$$

Defining a vector $\{\Lambda\}$ as being the ordered multipliers for the assemblage of $\mathrm{m}$ elements, and using equation (4.22) for the ordered elements in turn, a gradient matrix [N] is assembled for the system:

$$
\left\{\delta^{p}\right\}=[N]\{\Lambda\} \text {. }
$$

The matrix $[\mathbb{N}]$ is $k$ by $m$ and has in its first column the gradient vector $\left\{\frac{\partial \phi}{\partial \sigma}\right\}^{\prime}$ for the first element appearing in the first few rows; in its second column the vector $\left\{\frac{\partial \phi}{\partial \sigma}\right\}^{\prime}$ for the second element appearing in the next few rows, and so on. All other elements of [N] are zero.

In section (2.5) the idealization chosen for the scalar hardening coefficient $G$ is 


$$
\frac{I}{G}=E_{p}\left(\frac{\partial \phi}{\partial \sigma_{k \ell}} \frac{\partial \phi}{\partial \sigma_{k \ell}}\right) \text {, }
$$

but since the gradient vector has been normalized, this reduces to

$$
\frac{I}{G}=E_{p}
$$

Thus we introduce $a k$ by $k$ diagonal matrix $\left[D_{p}\right]$ whose non-zero entries consist of the terms $\frac{E_{p}}{V^{\prime}}$ for the ordered elements. The elastic-perfectly plastic case is recovered as the limit $\mathrm{E}_{\mathrm{p}} \rightarrow 0$.

Consider now the functional $\vec{U}_{p}^{0}$ of equation (3.26) and assume for the present that all elements of the assemblage undergo plastic deformation. Expressed in matrix form the volume integral of the functional $\overline{\mathrm{W}}^{\mathrm{O}}$ of equation $(3.20)$ is

$$
\begin{aligned}
\int_{V} \overline{\mathrm{W}}^{\mathrm{O}} d \mathrm{~V}= & \frac{1}{2}(\{\dot{\delta}\}-[\mathbb{N}]\{\Lambda\})^{\mathrm{T}}[\mathrm{D}](\{\dot{\delta}\}-[\mathrm{N}]\{\Lambda\}) \\
& +\frac{1}{2}\{\Lambda\}^{\mathrm{T}}\left[\mathrm{D}_{\mathrm{p}}\right]\{\Lambda\} .
\end{aligned}
$$

Velocities on the boundary of the assemblage can be expressed as linear functions of velocities at boundary nodes. Hence the rate form of potential energy of the boundary tractions $\mathrm{T}_{i}$ can be written in terms of a load rate vector $\{\dot{P}\}$ and boundary node velocities. The elements of $\{\dot{P}\}$ are calculated from

$$
\{\dot{\mathrm{u}}\}^{\mathrm{T}}\{\dot{\mathrm{P}}\}=\int_{\mathrm{S}_{\mathrm{T}}} \dot{\mathrm{T}}_{\dot{i}} \dot{\mathrm{u}}_{\dot{1}}^{*} \mathrm{dS}
$$

In the absence of body forces, substitution of equations (4.13), (4.26) and (4.27) in the expression for the functional $\overrightarrow{\mathrm{U}}_{\mathrm{p}}^{\mathrm{O}}$ (equation 3.26) yields 


$$
\begin{aligned}
\bar{U}_{p}^{O}= & \frac{1}{2}\{\dot{u}\}^{T}\left[B^{T} D B\right]\{\dot{u}\}-\frac{1}{2}\{\Lambda\}^{T}\left[B^{T} D N\right]\{\dot{u}\} \\
& -\frac{1}{2}\{\dot{u}\}^{T}\left[N^{T} D B\right]\{\Lambda\}+\frac{1}{2}\{\Lambda\}^{T}\left[N^{T} D N+D_{p}\right]\{\Lambda\}-\{\dot{u}\}^{T}\{\dot{P}\} .
\end{aligned}
$$

Introducing a column vector $\{\dot{u}: \Lambda\}$ comprising of $n$ elements of $\{\dot{u}\}$ and $m$ elements of $\{\Lambda\}$, this becomes

$$
\vec{U}_{p}^{0}=\frac{1}{2}\{\dot{u}: \Lambda\}^{T}\left[K^{*}\right]\{\dot{u}: \Lambda\}-\{\dot{u}: \Lambda\}^{T}\{\dot{P}: 0\},
$$

where the system matrix $\left[\mathrm{K}^{*}\right]$ is symmetric and given by

$$
\left[\mathrm{K}^{*}\right]=\left[\begin{array}{c:c}
{\left[\mathrm{B}^{\mathrm{T}} \mathrm{DB}\right]} & {\left[-\mathrm{B}^{\mathrm{T}} \mathrm{DN}\right]} \\
\hdashline\left[-\mathrm{N}^{\mathrm{T}} \mathrm{DB}\right] & {\left[\mathrm{N}^{\mathrm{T}} \mathrm{DN}+\mathrm{D}_{\mathrm{p}}\right]}
\end{array}\right] \text {. }
$$

The upper left submatrix $\left[\mathrm{B}^{\mathrm{T}} \mathrm{DB}\right]$ is the usual displacement method elastic system stiffness matrix. Indeed, if $\{\Lambda\} \equiv 0$, then $\bar{U}_{p}^{\circ}$ reduces to the rate form of potential energy for the systein.

The solution to a particular rate problem is thus given by that value of $\{\dot{u}: \Lambda\}$ which minimizes $\widetilde{J}_{p}^{O}$ in equation (4.29) subject to the constraints that for each element of $\{\Lambda\}$

$$
\begin{aligned}
& \Lambda=0 \text { if } \phi<0 \text { (elastic), } \\
& \text { and } \Lambda \geq 0 \text { if } \phi=0 \text { (plastic). }
\end{aligned}
$$

It is implicit that $\{\dot{P}\}$ is given, and that $\{\sigma\}$ and $\left\{\varepsilon^{p}\right\}$ are known for the assemblage so that the constraints (4.31) can be explicitly determined. 
Since $\vec{U}_{p}^{0}$ is homogeneous in the rates we may determine the solution to the incremental problem: minimize $\bar{U}_{p}^{0}$ with respect to $\{\Delta \mathrm{u}: \Lambda\}$, where

$$
\overline{\mathrm{U}}_{\mathrm{p}}^{\mathrm{O}}=\frac{1}{2}[\Delta \mathrm{u}: \Lambda\}^{\mathrm{T}}\left[\mathrm{K}^{*}\right]\{\Delta \mathrm{u}: \Lambda\}-\{\Delta \mathrm{u}: \Lambda\}^{\mathrm{T}}\{\Delta \mathrm{P}: 0\},
$$

subject to the constraints of equations (4.3I).

This constrained minimization problem can be stated as a formal mathematical programming problem and a general algorithm employed to determine the solution. Defining a vector $\{\phi\}$ corresponding to magnitudes of the yield function for the ordered elements, the quadratic programming problem is

$$
\min \left\{\bar{U}_{p}^{o}(\Delta u, \Lambda) \mid\{\Lambda\} \geq 0, \quad\{\phi\}^{T}\{\Lambda\}=0\right\}
$$

A quadratic functional is to be minimized subject to bounds (nonnegativity constraints on $\{\Lambda\}$ ) and linear constraints to ensure $\Lambda$ is zero if $\phi<0$.

The state-of-the-art in general quadratic programing algorithms is such that computer running times increase significantly with increase in numbers of variables and constraints. Moreover this problem must be solved for each load increment and hence computation time for any realistic continuum problem becomes prohibitive. Fortunately because of the nature of the minimization problem and its constraints, it is possible to develop an efficient intuitive solution algorithm which does not rely on formal mathematical programming techniques. This will be presented in the following section. 


\subsection{An Algorithm for the Minimization of $\bar{U}_{p}^{\circ}$}

In Martin and Reddy's [24] application of the minimum principle to the truss problem, an algorithm was suggested for solution of the programming problem. This algorithm may be extended to the continuum finite element formulation.

Consider the assemblage of elements at an arbitrary stage in the loading program when the load on the structure, which was initially in the virgin state, is $\{P\}$. Further, let $\{P\}$ be such that at least part of the structure is plastic (i.e. some elements in $v_{p}$ ). The displacements, elastic strains, plastic strains and stresses of the assemblage corresponding to the load $\{P\}$ are known.

For the solution to the next load increment $\{\Delta \mathrm{P}\}$ we are required to minimize $\overline{\mathrm{U}}_{\mathrm{p}}^{0}$ (equation 4.32 ) subject to the constraints of equations (4.31). Those elements governed by constraint (4.31a) are elastic (ie in $V_{e}$ ) and are easily identified. However for those elements which are plastic and are therefore governed by constraints (4.31b) we do not know a priori which will load or which will unload.

If all elements of the vector $\{\Delta u: \Lambda\}$ are non-zero the least value of the quadratic functional $\bar{U}_{p}^{O}$ is given by solution of the set of simultaneous linear equations

$$
\left[K^{*}\right]\{\Delta u: \Lambda\}=\{P: O\}
$$

This suggests an algorithm for the minimization of $\overline{\mathrm{U}}_{\mathrm{p}}^{\circ}$ based on an initial guess that elements in $\mathrm{V}_{\mathrm{p}}$ are such that $\Lambda=0$ or $\Lambda \neq 0$. We proceed as follows. Identifying all elements for which $\phi<0$, and those elements for which $\phi=0$ and in which unloading is guessed to occur, we eliminate corresponding rows and columns of $\left[K^{*}\right]$. 
The remaining simultaneous linear equations (4.34) are then solved for $\{\Delta u\}$ and proposed non-zero elements of $\{\Lambda\}$. This represents a trial solution for $\{\Delta u\}$ and $\{\Lambda\}$.

Necessary and sufficient conditions for the solution to be correct are: elements of $\{\Lambda\}$ are non-negative if they were assumed to be non-zero, and $\frac{\partial \phi}{\partial \sigma_{i j}} \Delta \sigma_{i j}<0$ for those elements for which $\phi=0$ but were guessed to be unloading. If these checks are not satisfied the solution has not been found and we must revise the choice of elements in $V_{p}$ for which we guess $\Lambda$ is zero, and re-solve equations (4.34). In revising the choice of loading or unloading finite elements in $V_{p}$ we consider those elements of $\{\Lambda\}$ which did not satisfy the constraints of equations (4.31b). If on one hand $A$ was assumed to be zero and $\frac{\partial \phi}{\partial \sigma_{i j}} \Delta \sigma_{i j}>0$, this element of $\{\Lambda\}$ is now assumed to be nonzero. On the other hand if $\Lambda$ was assumed to be non-zero and $\Lambda$ is negative, it is now assumed to be zero.

Convergence of the iterative procedure has not been conclusively proved, but experience indicates that it is rapid and fails only as the limit load is approached in the elastic-perfectly plastic case. Further, it will be shown in the following section that the formulation and minimization algorithm can be reduced to the conventional tangent modulus approach. If loads are increased monotonically unloading of elements very seldom occurs, and thus the best initial guess in the iterative procedure is that $\Lambda$ is non-zero for all elements in $V_{p}$. This initial assumption was also used for non-monotonic loading, in which case the algorithm was found to converge within one or two iterations. 
4.4 Reduction to Tangent Modulus Approach

Consider an arbitrary stage in the loading program at which some finite elements are plastic. For the next load increment the system matrix [ $\left.\mathrm{K}^{*}\right]$ is evaluated and, following the minimization algorithm, particular rows and columns are eliminated corresponding to elastic elements, or plastic elements guessed to unload. To perform this elimination process it is convenient to consider these rows and columns as set to zero and unity inserted on the leading diagonal, thus retaining the original dimension of $\left[\mathrm{K}^{*}\right]$.

Let the system matrix corresponding to the correct solution be $\left[\bar{K}^{*}\right]$. That is, the solution of the equations

$$
\left[\overline{K^{*}}\right]\{\Delta \mathrm{u}: \Lambda\}=\{\Delta \mathrm{P}: 0\}
$$

is $\{\Delta u: \Lambda\}=\{\Delta \bar{u}: \bar{\Lambda}\}$ where $\{\Delta \bar{u}: \bar{\Lambda}\}$ satisfy the constraints of equations (4.31). Expanding $\left[\overline{\mathrm{K}}^{*}\right]$ in equation (4.35) this becomes

$$
\left[\begin{array}{c|c}
B^{T} D B & -B^{T} D N \\
\hdashline-N^{T} D B & N^{T} D N+D_{p}
\end{array}\right]\left\{\begin{array}{c}
\Delta u \\
\Lambda
\end{array}\right\}=\left\{\begin{array}{c}
\Delta \mathrm{P} \\
0
\end{array}\right\},
$$

with certain rows and columns set to zero.

$$
\begin{aligned}
& \text { Partitioning equations }(4.36) \text { gives } \\
& {\left[-N^{T} D B\right]\{\Delta u\}+\left[N^{T} D N+D_{p}\right]\{\Lambda\}=\{0\} .}
\end{aligned}
$$

Since $[D]$ and $\left[D_{p}\right]$ are positive definite it follows that 


$$
\{\Lambda\}=\left[N^{T} D N+D_{p}\right]^{-1}\left[N^{T} D B\right]\{\Delta u\}
$$

Substituting for $\{\Lambda\}$ in the remainder of the partitioned equations (4.36) gives

$$
[B]^{\mathrm{T}}[\overline{\mathrm{D}}][\mathrm{B}]\{\Delta \mathrm{u}\}=\{\Delta \mathrm{P}\}
$$

where $[\bar{D}]=\left[D-D N\left(N^{T} D N+D_{p}\right)^{-1} N^{T} D\right]$

This stress-strain matrix $[\bar{D}]$ reflects the current constitutive relations for each element of the assemblage. Solving equation (4.39) will yield $\{\Delta u\}=\{\Delta \bar{u}\}$.

Investigating the form of $[\bar{D}]$ we see that like the elastic system elasticity matrix $[D],[\bar{D}]$ is also symmetric and composed of submatrices along a diagonal band. Each matrix corresponds to an element of the assemblage. For the ith element, these submatrices are $\frac{I}{V},\left[D^{\prime}\right]_{i}$ and $\frac{i}{V},\left[\bar{D}^{\prime}\right]_{i}$, and the normalized gradient vector is $\left\{\frac{\partial \phi}{\partial \sigma}\right\}_{i}^{\prime}$. Consider this ith element of the assemblage. If it was elastic, or plastic and unloading, $\Lambda_{i}$ was assumed zero and the corresponding row and column of $\left[\mathrm{K}^{*}\right]$ set to zero. Evaluating equation (4.40) will result in

$$
\left[\bar{D}^{\prime}\right]_{i}=\left[D^{\prime}\right]_{i}
$$

If $\Lambda_{i}$ was assumed non zero the ith submatrix of $[\bar{D}]$ in equation (4.40) is equivalent to 


$$
\left[\bar{D}^{\prime}\right]_{i}=\left[\left[D^{\prime}\right]-\frac{\left[D^{\prime}\right]\left\{\frac{\partial \phi}{\partial \sigma}\right\}^{\prime}\left\{\frac{\partial \sigma}{\partial \sigma}\right\}^{\prime T}\left[D^{\prime}\right]}{E_{p}+\left\{\frac{\partial \phi}{\partial \sigma}\right\}^{\prime T}\left[D^{\prime}\right]\left\{\frac{\partial \phi}{\partial \sigma}\right\}^{\prime}}\right]_{i}
$$

which is in an identical form to the element elasto-plastic matrix [D] $]_{\text {ep }}^{*}$ derived by Zienkiewicz et al [11] for the conventional tangent modulus approach.

In following the steps of the tangent modulus approach a block-diagonal matrix $\left[D_{\mathrm{T}}\right]$ is assembled for the system by considering each finite element in turn and using equation (4.41) if the element is elastic, or plastic and guessed to unload; and equation (4.42) if the element is plastic and guessed to load. Having assembled $\left[D_{T}\right]$, a solution for $\{\Delta u\}$ is obtained from the equation

$$
[B]^{\mathrm{T}}\left[D_{T}\right][B]\{\Delta \mathrm{u}\}=\{\Delta \mathrm{P}\}
$$

and $\Delta \sigma_{i f}$ evaluated for each plastic element. The solution is correct if for each element in $\mathrm{V}_{p}: \frac{\partial \phi}{\partial \sigma_{i j}} \Delta \sigma_{i j}<0$ for elements guessed to unload, and $\frac{\partial \phi}{\partial \sigma_{i j}} \Delta \sigma_{i j} \geq 0$ for elements guessed, to load. If these constraints are not satisfied new assumptions are made as to loading and unloading plastic elements and a new tangent modulus matrix $\left[D_{\mathrm{T}}\right]$ assembled. The process is then repeated.

In the programming approach a solution is correct if: $\Lambda=0$ for elastic elements; $\frac{\partial \phi}{\partial \sigma_{i j}} \Delta \sigma_{i j}<0$ for plastic elements assumed to unload; and $\Lambda \geq 0$ for plastic elements assumed to load. This non-negativity constraint on $\Lambda$ is identical to the constraint on the sign $\frac{\partial \phi}{\partial \sigma_{i j}} \Delta \sigma_{i j}$ for loading plastic elements in the tangent modulus approach. 
Recalling equation (2.10)

$$
\lambda=G \frac{\partial \phi}{\partial \sigma_{k l}} d \sigma_{k l},
$$

and since $G$ is positive definite,

$$
\Lambda \geq 0 \longleftrightarrow \frac{\partial \phi}{\partial \sigma_{i j}} \Delta \sigma_{i j} \geq 0 .
$$

We have thus shown that the solution algorithm suggested for the kinematic minimum principle reduces to the conventional tangent modulus approach for incremental elasto-plastic analysis. The matrix [D] is identical to $\left[D_{T}\right]$, and is a tangent modulus stress-strain matrix to account for modified stiffness under loading.

To the writer's knowledge convergence of the conventional tangent moduIus algorithm has not been proved, although it is widely accepted and used, [14], [15], [28]. Hence reduction of the solution algorithm suggested for the quadratic programming problem to the tangent modulus approach certainly provides a measure of confidence in the iterative procedure suggested in the preceding section.

\subsection{Solution Procedure for the Incremental Problem}

In presenting the application of the minimum principle to constant strain finite elements we have discussed the evaluation of the system matrix [ $\left.\mathrm{K}^{*}\right]$ on a global basis. In so doing all $\mathrm{m}$ elements of the assemblage are represented in each submatrix of $\left[K^{*}\right]$, resulting in an $(n+m)$ square system matrix. Then, following the algorithm for minimizing $\bar{U}_{p}^{\circ}$, rows and columns corresponding to elastic elements and unloading plastic elements are deleted and a solution obtained. 
In the computer implementation of the minimum principle both computational effort and computer storage requirements can be substantially reduced by evaluating only relevant element submatrices of $\left[\mathrm{K}^{*}\right]$.

Further, for each load increment only part of $\left[\mathrm{K}^{*}\right]$ need be reformulated. As noted previously the $\left[\mathrm{B}^{\mathrm{T}} \mathrm{DB}\right]$ submatrix is the usual displacement method elastic system stiffness matrix and remains unchanged for each determination of $\left[\mathrm{K}^{*}\right]$. However, the remaining submatrices of $\left[\mathrm{K}^{*}\right]$ must be calculated for each load increment since the gradient matrix $[N]$ depends on current stress, and in the kinematic hardening case, current plastic strain.

Instead of the global approach presented above we take each element in turn and evaluate the element deformation matrix [ $\left.B^{\prime}\right]_{i}$, $(i=1,2, \ldots, m)$. These matrices are stored for later use. Each six by six elastic element stiffness matrix $\left[B^{T} D B\right]_{i}^{i}(i=1,2, \ldots, m)$ is explicitly evaluated and assembled in appropriate positions corresponding to element node displacements, to form the $\mathrm{n}$ by $\mathrm{n}$ elastic system stiffness matrix $\left[B^{T} D B\right]$. This is stored so that it may be retrieved for each reformulation of $\left[K^{*}\right]$.

The body is assumed to be initially in the virgin state and therefore all elements of the assemblage are elastic. Thus, since $\{\Lambda\} \equiv 0$, the solution of the first load increment corresponds to a linear elastic analysis

$$
\left[B^{T} D B\right]\{\Delta u\}=\{\Delta P\}
$$

where $\{\Delta \mathrm{P}\}$ need give only relative magnitudes of components of the load vector. On the basis of this solution relative magnitudes of stress components are calculated for each element, and the smallest load factor determined to cause at least one element to enter $v_{p}$. 
(Fortuitously or through symmetry more than one element may correspond to the smallest load factor). For the assemblage, increments of stress, strain, load and displacement are scaled by this load factor and become current totals of each respective quantity. All plastic strains are zero but for the following load increment there is at least one plastic element.

Consider this as representing an arbitrary stage in the loading program when part of the structure is plastic (i.e. some elements in $\mathrm{V}_{\mathrm{p}}$ ). The system matrix $\left[\mathrm{K}^{*}\right]$ is required for the following load increment. Firstly, the elastic system matrix $\left[\mathrm{B}^{\mathrm{T}} \mathrm{DB}\right]$ is retrieved from storage and entered into $\left[\mathrm{K}^{*}\right]$. To determine the remainder of the symmetric system matrix only terms on and above the leading diagonal are evaluated. Recall that the best initial guess for the solution algorithm is that $\Lambda \neq 0$ for all elements in $V_{p}$. Beginning with [K*] corresponding to the elastic matrix $\left[B^{T} D B\right]$, each element is considered in turn, and if plastic the next row and column of [ $\left.\mathrm{K}^{*}\right]$ assigned to it. For each of these elements in $V_{p}$ a six by one element matrix $\left\{-\left[B^{\prime}\right]^{T}\left[D^{\prime}\right]\left\{\frac{\partial \phi}{\partial \sigma}\right\}^{\prime}\right\}$ and scalar $\frac{1}{V^{\prime}}\left(\left\{\frac{\partial \phi}{\partial \sigma}\right\}^{\prime T}\left[D^{\prime}\right]\left\{\frac{\partial \phi}{\partial \sigma^{\prime}}\right\}^{\prime}+E_{p}\right)$ is evaluated and entered into the appropriate positions of its assigned column to form the upper triangle of $\left[\mathrm{K}^{*}\right]$.

Thus a condensed system matrix is produced wherein the number of columns in the $\left[-\mathrm{B}^{\mathrm{T}} \mathrm{DN}\right]$ submatrix is equal to the number of elements currently in $\mathrm{V}_{\mathrm{p}}$. The plastic submatrices of $\left[\mathrm{K}^{*}\right]$ are also stored in case of iteration within a load increment, in which case rows and columns are deleted corresponding to unloading plastic elements. Having determined a satisfactory solution to the current load increment, totals of stress, strain, load and displacement are updated and $\left[\mathrm{K}^{*}\right]$ determined for the succeeding load increment. 
In this way the magnitude of each load increment is determined by the lowest load multiplier which causes an elastic element to yield. Without significant loss of accuracy the total number of load increments is decreased by including in $\mathrm{V}_{\mathrm{p}}$ elements close to yielding, and correcting stresses after the load increment. Further, symmetry and sparseness of the system matrix may be explofted and an efficient storage scheme and associated solution routine employed. These computational details will be described in Chapter 5 .

\subsection{Application to Higher Order Finite Elements}

The use of constant strain finite elements in the application of the minimum principle to continuum problems requires discretization of the body into a large number of elements (especially in regions where plastic deformations occur), if results of an analysis are to be meaningful. This in turn implies a large number of displacement degrees of freedom, and since an additional degree of freedom corresponds to each loading plastic element, computer storage requirements and computational effort increase significantly when large regions of the body are plastic.

A saving of storage requirements and computational effort can be achieved by use of higher order finite elements. Again limiting discussion to the cases of plane stress, plane strain and axial symmetry, we shall in particular consider use of cubic quadrilateral isoparametric finite elements, [29].

Since each of these elements is connected to twelve nodes (one at each corner and two on each side), a cubic variation of the displacement field is defined across each element. This implies quadratic variation of strain within an element and thus the strain displacement relations [ $B^{\prime}$ ] are no longer constant. In the case of 
linear elastic analysis, integration of the strain field cannot be performed explicitly, necessitating use of a numerical integration technique such as Gauss quadrature. This technique is further exploited in the elasto-plastic case.

Discretizing a continuum into an assemblage of cubic quadrilateral isoparametric finite elements consider a generic element lying in the $x, y$ plane of a global Cartesian coordinate system $x, y, z$, as shown in Figure 4.1 A natural coordinate system $r$, $s$ is defined on the element as shown, where $-1 \leq r \leq 1$, $-1 \leq \mathrm{s} \leq 1$. Coordinates $\mathrm{x}, \mathrm{y}$ of any point within the element are

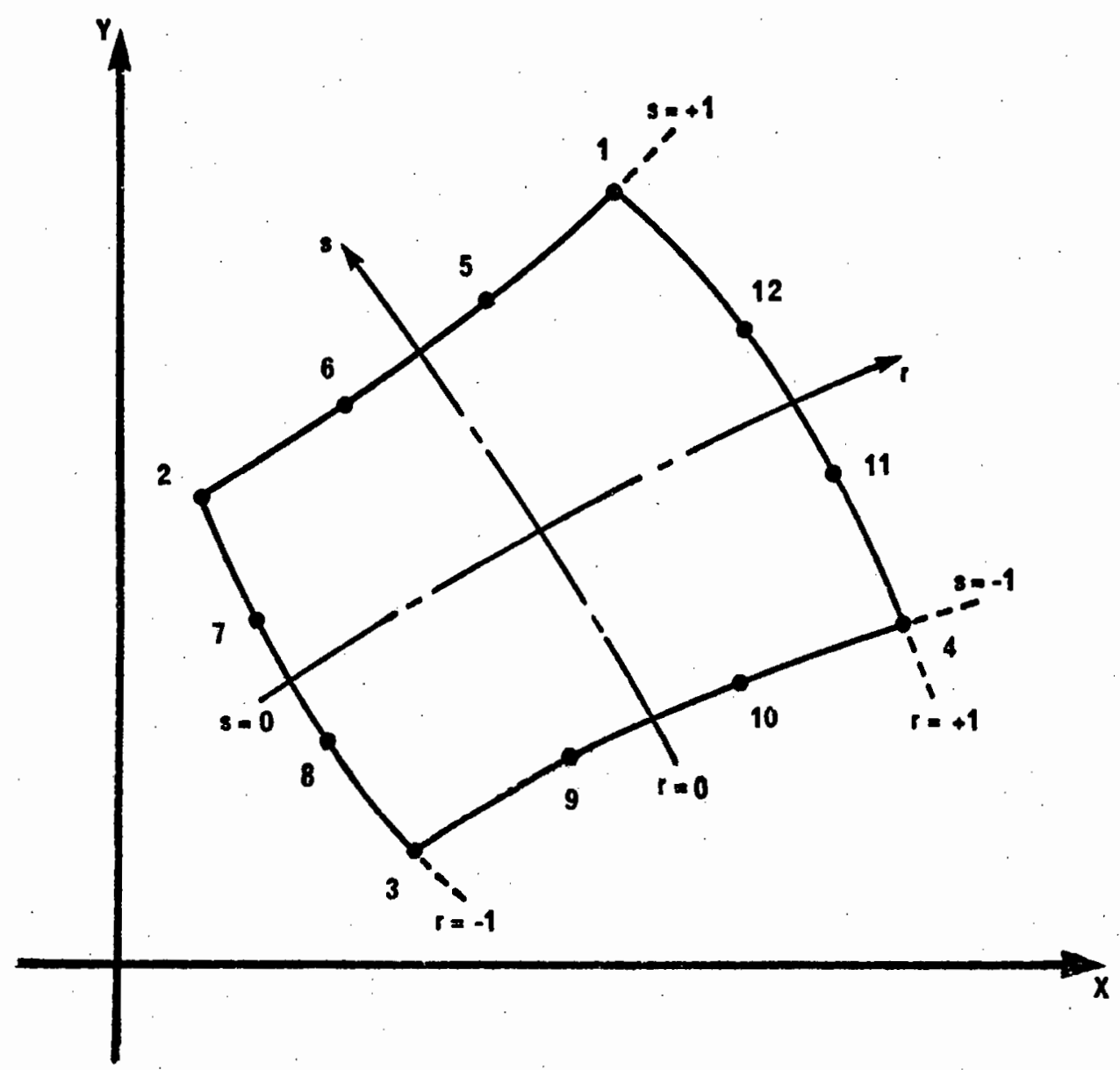

Figure 4.1 A cubic quadrilateral isoparametric finite element 
described in terms of element node coordinates by means of shape functions. A shape function $h_{i}$ is associated with each node of the element and has unit value at that node and zero value at all other element nodes. The coordinate interpolations are

$$
x=\sum_{i=1}^{12} h_{i} x_{i}, \quad y=\sum_{i=1}^{12} h_{i} y_{i},
$$

where $x_{i}, y_{i}(i=1,2, \ldots, 12)$ are the global coordinates of the twelve nodes of the element.

The shape functions $h_{i}$ are defined in terms of the natural coordinate system of the element, which has variables $r$, s that vary from - 1 to +1 . Defining the quantities $r_{0}=r r_{i}$ and $s_{0}=s s_{1}$ for the $i$ th node of the element, then for the corner nodes $(i=1,2,3,4)$ the shape functions are

$$
h_{i}=\frac{1}{32}\left(1+r_{0}\right)\left(1+s_{0}\right)\left\{-10+9\left(r^{2}+s^{2}\right)\right\}
$$

For the remaining element nodes $(i=5,6, \ldots, 12)$

$$
\begin{aligned}
h_{i}= & \frac{9}{32}\left(1+r_{0}\right)\left(1-s^{2}\right)\left(1+9 s_{0}\right) \\
& \text { for }\left(r_{i}, s_{i}\right)=\left(-1,-\frac{1}{3}\right),\left(1,-\frac{1}{3}\right),\left(-1, \frac{1}{3}\right) \text { and }\left(1, \frac{1}{3}\right) ;
\end{aligned}
$$

and

$$
\begin{aligned}
h_{i}= & \frac{9}{32}\left(1+s_{0}\right)\left(1-r^{2}\right)\left(1+9 r_{0}\right) \\
& \text { for }\left(r_{i}, s_{i}\right)=\left(-\frac{1}{3},-1\right),\left(\frac{1}{3},-1\right),\left(-\frac{1}{3}, 1\right) \text { and }\left(\frac{1}{3}, 1\right) .
\end{aligned}
$$

In view of equations $(4.45)$ and $(4.46)$ an advantage of the isoparametric formulation is apparent in that elements can have curved boundaries. 
The basis of the isoparametric formulation is that the shape functions $h_{i}$ used to describe geometry are also used to describe displacements at any point in the element in terms of displacements at the element nodes. Thus

$$
u=\sum_{i=1}^{12} h_{i} u_{i}, \quad v=\sum_{i=1}^{12} h_{i} v_{i}
$$

where $u, v$ are displacements respectively in the $x, y$ directions at a point in the element, and $u_{i}, v_{i}$ are the element node displacement components. As a consequence of $(4.45)$ and $(4.47)$ the cubic quadri-lateral isoparametric element is both compatible and complete, [29]. Element strains are obtained in terms of derivatives of displacements with respect to global coordinate directions. But, because element displacements are defined in the natural coordinate system (equations 4.47 ), the inverse of the Jacobian operator is used to relate global coordinate derivatives to natural coordinate derivatives:

$$
\left\{\begin{array}{l}
\frac{\partial}{\partial x} \\
\frac{\partial}{\partial y}
\end{array}\right\}=J^{-1}\left\{\begin{array}{l}
\frac{\partial}{\partial r} \\
\frac{\partial}{\partial s}
\end{array}\right\},
$$

where $J=\left[\begin{array}{ll}\frac{\partial x}{\partial r} & \frac{\partial y}{\partial r} \\ \frac{\partial x}{\partial s} & \frac{\partial y}{\partial s}\end{array}\right]$.

The inverse of $J$ exists provided there is a unique correspondence between natural and global coordinates for the element. Using equations (2.4), (4.47) and (4.48) element strains $\varepsilon_{i j}$ are obtained from element node displacements $u_{k}$ for any point within the element: 


$$
\varepsilon_{i j}(r, s)=B_{i j k}(r, s) u_{k}
$$

where the subscripts $i, j$ refer to the relevant strain components for plane stress, plane strain or axial symmetry. As before in the case of plane strain elements of $B_{i j k}$ corresponding to $\varepsilon_{z z}$ are zero. The element elastic stiffness matrix is

$$
\left[k^{\prime}\right]=\int_{V^{\prime}} B_{i j} D_{i j k \ell} B_{k \ell} d V,
$$

where the integration extends over the element volume. Writing this in matrix notation

$$
\left[k^{\prime}\right]=\int_{V^{\prime}}\left[B^{T} D B\right]^{\prime} \cdot d V .
$$

Since the elements of [ $\left.\mathrm{B}^{\prime}\right]$ are functions of the natural coordinates $r, s$ the: volume integration is performed over the natural coordinate volume by writing the element volume differential dV in terms of $r, s$. This is

$$
d V=t \operatorname{det} J d r d s
$$

where $\operatorname{det} J$ is the determinant of the Jacobian operator (equation 4.49). In the case of plane stress $t$ is the element thickness, in plane strain $t$ is unity, and in the axisymmetric case $t$ is $2 \pi$ times the radius from the axis of symmetry to the point $(r, s)$.

In general the inverse of $J$ in equation (4.48) and the integral in equation (4.52) cannot be explicitly evaluated and thus numerical integration must be used. The twenty-four by twenty-four element elastic stiffness matrix is evaluated as 
anywhere in the assemblage. In a manner which is analogous to the treatment of constant strain finite elements discussed previously, the elastic-plastic interface is determined by evaluating the yield function at each Gauss point of the assemblage and on this basis dividing the body into an elastic domain $\mathrm{V}_{\mathrm{e}}$ where $\phi<0$, and a plastic domain $\mathrm{V}_{\mathrm{p}}$ where $\phi=0$.

To formulate the minimum principle the functional $\overline{\mathrm{W}}^{\circ}$ of equation (3.20) is required, integrated over the body. Defining a functional $\overline{\mathrm{W}}_{\mathrm{q}}^{\mathrm{q}}$ for the qth element of the assemblage as

$$
\overline{\mathrm{W}}_{\mathrm{q}}^{\mathrm{O}}=\frac{1}{2} D_{i j k \ell}\left(\dot{\varepsilon}_{i j}-\lambda \frac{\partial \phi}{\partial \sigma_{i j}}\right)\left(\dot{\varepsilon}_{k \ell}-\lambda \frac{\partial \phi}{\partial \sigma_{k \ell}}\right)+\frac{\lambda^{2}}{2 G},
$$

it follows from the discretization of the body into m elements that

$$
\int_{V} \bar{W}^{O} d V=\sum_{q=I}^{m} \int_{V_{q}^{\prime}} \bar{W}_{q}^{O} d V
$$

where $V$ is the volume of the body and $V_{q}^{\prime}$ is the volume of the qth element of the assemblage.

Substituting the rate form of the strain-displacement relations (4.50) into the expression for $\overline{\mathrm{W}}_{\mathrm{q}}^{0}$ gives in matrix form for the qth element

$$
\bar{W}_{q}^{O}=\frac{1}{2}\left(\left[B^{\prime}\right]\left\{\dot{u}^{\prime}\right\}-\lambda\left\{\frac{\partial \phi}{\partial \sigma}\right\}^{\prime}\right)^{T}\left[D^{\prime}\right]\left(\left[B^{\prime}\right]\left\{\dot{u}^{\prime}\right\}-\lambda\left\{\frac{\partial \phi}{\partial \sigma}\right\}^{\prime}\right)+\frac{\lambda^{2}}{2 G}
$$

where $\left\{\dot{u}^{\prime}\right\}$ are element node velocites, and [ $\left.B^{\prime}\right], \lambda$ and $\left\{\frac{\partial \phi}{\partial \sigma}\right\}^{\prime}$ are functions of the element natural coordinates $r, s$.

Exploiting the numerical integration technique, a plastic multiplier $\lambda$ is associated with each Gauss point of the body, so that the plastic strain rate for the eth Gauss point is given by 


$$
\left\{\varepsilon^{p}\right\}_{\ell}^{\prime}=\lambda_{\ell}\left\{\frac{\partial \phi}{\partial \sigma}\right\}_{\ell}^{\prime}
$$

Hence, using Gauss quadrature to integrate equation (4.57) yields

$$
\int_{V_{q}^{\prime}} \bar{W}_{q}^{\circ} d V=\sum_{i, j} \alpha_{i j} \bar{W}_{q}^{o}\left(r_{i}, s_{j}\right) t \operatorname{det} J
$$

and substituting this in equation (4.56) we have finally

$$
\int_{V} \bar{W}^{\circ} d V=\sum_{q=1}^{m} \sum_{i, j} \alpha_{i j} \bar{W}_{q}^{\circ}\left(r_{i}, s_{j}\right) t \operatorname{det} J .
$$

To formulate $\bar{U}_{p}^{\circ}$ the rate form of the potential energy of the boundary tractions is required. In the isoparametric formulation velocities along the boundaries of an element are described directly by the shape functions and element node velocities. Thus the rate form of the potential energy of the traction rates $\dot{T}(r, s)$ can be written in terms of an element nodal load rate vector $\left\{\dot{P}^{\prime}\right\}$ and the element node velocities $\{\dot{u}\}$ as

$$
\left\{\dot{u}^{\prime}\right\}^{T}\left\{\dot{P}^{\prime}\right\}=\int_{S_{T}^{\prime}} \dot{u}(r, \dot{s}) \dot{T}(r, s) d s .
$$

In general numerical integration must be used to evaluate the integral. Using Gauss quadrature the expression for $\left\{\dot{P}^{\prime}\right\}$ becomes

$$
\left\{\dot{P}^{\prime}\right\}=\sum_{i, j} \alpha_{i j}\left(\sum_{\rho=1}^{12} H_{\rho}\left(r_{i}, s_{j}\right) \dot{T}\left(r_{i}, s_{j}\right)\right) t \operatorname{det} J,
$$

where $H$ is a vector of shape functions. The global load rate vector $\left\{\dot{P}^{\prime}\right\}$ is obtained by summing contributions from each element nodal load rate vector in appropriate positions corresponding to the ordered velocity vector $\{\dot{u}\}$ for the assemblage. 
In the absence of body forces the minimum principle for the assemblage of isoparametric elements becomes:

$$
\operatorname{minimize} \overline{\mathrm{U}}_{\mathrm{p}}^{\circ}=\int_{V} \overline{\mathrm{W}}^{\circ} \mathrm{dV}-\{\dot{\mathrm{u}}\}^{\mathrm{T}}\{\dot{\mathrm{P}}\}
$$

subject to the constraints $\lambda=0$ in $\mathrm{V}_{\mathrm{e}}$ and $\lambda \geq 0$ in $\mathrm{V}_{\mathrm{p}}$. In a manner similar to the formulation for constant strain elements, a system matrix [K*] corresponding to all possible degrees of freedom may be assembled from equation (4.63). Since a plastic multiplier $\lambda$ is associated with each Gauss point of the body, the size of this system matrix will be $(n+9 m)$ square. As before the governing equations are homogeneous in the rates and hence a solution may be determined for a load increment $\{\Delta \mathrm{P}\}$ by employing the minimization algorithm to delete appropriate rows and columns. In so doing each Gauss point of the body is considered in an analogous manner to the treatment of each constant strain element, as described in the minimization algorithm given in section $(4 \cdot 3)$.

The global formulation for the isoparametric problem has been presented for the sake of completeness and for comparison with the global constant strain formulation. In practice the full $(n+9 m)$ square system matrix $\left[\mathrm{K}^{*}\right]$ is not evaluated. We proceed as follows: at the start of the analysis problem the elastic system stiffness matrix $\left[\mathrm{B}^{\mathrm{T}} \mathrm{DB}\right]$ is evaluated and stored. Since all integration points are in $\mathrm{V}_{e}$ the first load increment corresponds to a linear elastic analysis. These results are scaled by a load factor just large enough to ensure $\phi=0$ for at least one integration point. 
At an arbitrary stage in the loading when part of the body is plastic we wish to assemble the system matrix. As before the elastic system matrix is retrieved from storage and entered into $\left[\mathrm{K}^{*}\right]$. Considering each Gauss point of the assemblage in turn we identify those points in $V_{p}$ and, beginning from $\left[\mathrm{K}^{*}\right] \equiv\left[\mathrm{B}^{\mathrm{T}} \mathrm{DB}\right]$, assign an additional column and row of $\left[\mathrm{K}^{*}\right]$ to each plastic integration point. For the eth plastic point the terms to be entered in appropriate positions of the eth additional column are evaluated as the vector $\left\{\alpha_{i j}\left[B^{\prime}\right]^{T}\left[D^{\prime}\right]\left\{\frac{\partial \phi}{\partial \sigma}\right\}^{\prime} t \operatorname{det} J\right\}$ and scalar $\alpha_{i j}\left(\left\{\frac{\partial \phi}{\partial \sigma}\right\}^{\prime T}\left[D^{\prime}\right]\left\{\frac{\partial \phi}{\partial \sigma}\right\}^{\prime}+E_{p}\right) t \operatorname{det} J$, where $\left[B^{\prime}\right],\left\{\frac{\partial \phi}{\partial \sigma}\right\}^{\prime}, \alpha_{i j}, t$ and det $J$ are evaluated at the point $\ell$. Having assembled the condensed system matrix the solution algorithm may proceed as described for the constant strain finite elements but analogously we consider each integration point instead of each element.

Provided the isoparametric element discretization is not too coarse, it is found that a further saving in computer storage and computation can be achieved if only one column of the $\left[-B^{T} D N\right]$ and $\left[\mathrm{N}^{\mathrm{T}} \mathrm{DN}+\mathrm{D}_{\mathrm{p}}\right]$ submatrices is assigned to each plastic or partially plastic finite element, and contributions from all plastic integration points within that element added into this single column. Thus whether an element is totally plastic or an elastic-plastic interface occurs within the element a single 'average' value of plastic multiplier $\lambda$ is associated with all non-zero plastic strains within the element. In plastic regions stress gradients are generally small, especially in the case of no strain hardening. It follows that variation in yield function gradient (and thus plastic strain direction) across the plastic domain will also be of the same order. The effect therefore, of associating only one plastic multiplier with each plastic or partially plastic element is to average out plastic strain magnitudes across the 
element. However employing this device results in large savings in computation and computer storage, while still providing a reasonable approximate solution to the problem.

Illustrative numerical examples will be presented in Chapter 6 for both constant strain finite element analyses and cubic quadrilateral isoparametric finite element analyses. In the following Chapter solution techniques and computational details will first be given. 
C H A P T E R 5

SOLUTION TECHNIQUES, UNIQUENESS AND

SOME NOTES ON THE COMPUTATIONAL PROCEDURE

\section{$5.1 \quad$ Introduction}

The structure of the system matrix [K*] may be exploited to minimize computer storage requirements and reduce computational effort.

Consider solution of the system of simultaneous linear equations (4.34). Let there be $\mathrm{n}$ displacement increment variables and at an arbitrary stage in the loading history, p plastic multiplier variables. Since only relevant element submatrices of [ $\left.\mathrm{K}^{*}\right]$ are evaluated the system matrix is $(n+p)$ square. $\left[\mathrm{K}^{*}\right]$ is symmetric, and the $n$ by $n$ submatrix $\left[\mathrm{B}^{\mathrm{T}} \mathrm{DB}\right.$ ] is banded, the band-width depending on the nodal numbering of the assemblage. Let the half band-width be w. In general, employing a judicious nodal numbering scheme, $\mathrm{w} \ll \mathrm{n}$. The $p$ by $p$ submatrix $\left[N^{T} D N+D_{p}\right]$ is diagonal, while the $n$ by $p$ submatrix $\left[-B^{T} D N\right]$ is sparse but of no fixed form. We may depict the set of $(n+p)$ simultaneous linear equations as follows:
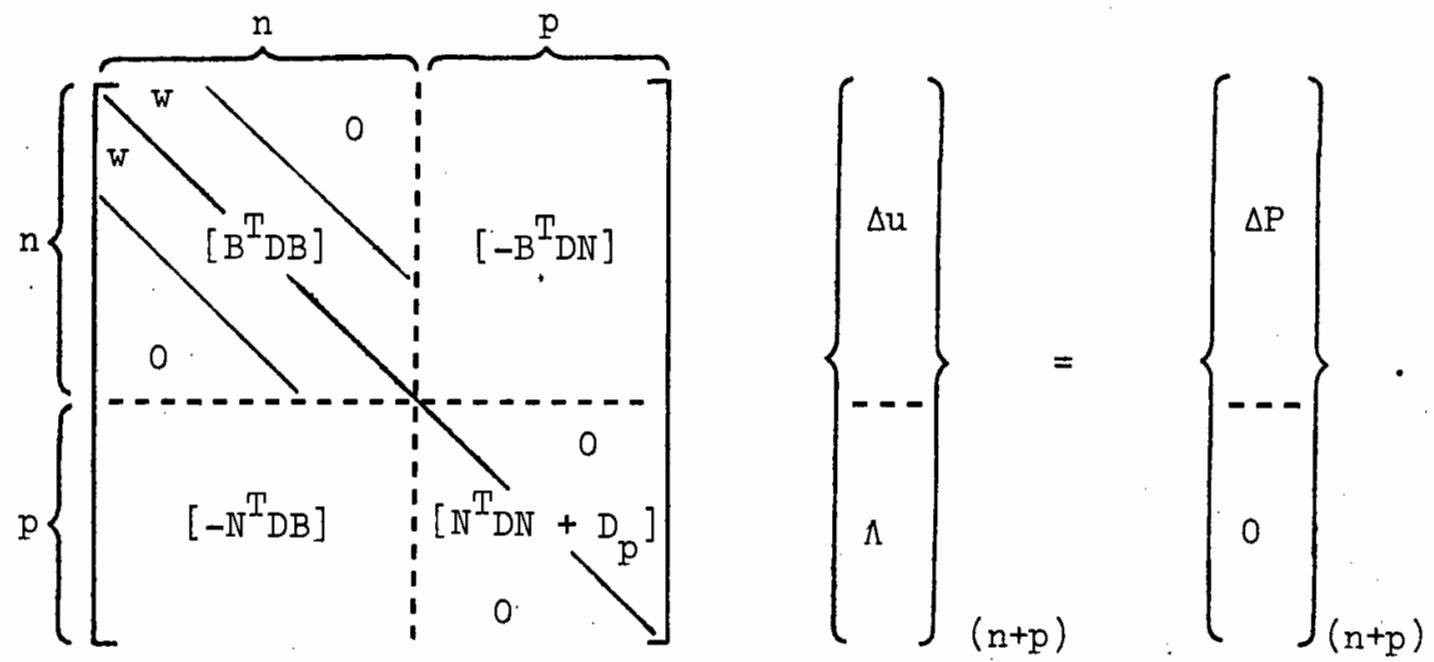
The symmetry and sparseness of $\left[\mathrm{K}^{*}\right]$ permit an efficient computer storage scheme:

(i) only half the band of the elastic system stiffness matrix $\left[B^{T} D B\right]$ is evaluated. As this submatrix is identical for all system matrices $\left[\mathrm{K}^{*}\right]$ throughout the loading program, it is stored on a peripheral storage device (magnetic-drum, -disc or -tape) and retrieved at the start of each load increment.

(ii) the submatrix $\left[-B^{\mathrm{T}} \mathrm{DN}\right]$ must be recalculated for each load increment as entries depend on current yield function gradients for those stress points on the yield surface. For each load increment this submatrix is stored on a peripheral device as unloading of stress points may occur necessitating iteration within a load increment. (iii) the submatrix $\left[N^{T} D N+D_{p}\right]$ is also dependent on current stresses and is therefore evaluated for each increment, but since it is diagonal only the diagonal elements are stored.

The minimum storage required to evaluate the $(n+p)$ unknowns 'in core' is that required in (i), (ii) and (iii) above, plus an additional work area of size $\mathrm{p}$ by $\mathrm{p}$. This gives a total core requirement of $\left(n w+(n+1) p+p^{2}\right)$ for $\left[K^{*}\right]$. In general $w \ll n$ and $p$ varies from zero according to development of plastic regions in the body. However, in the analysis of realistic problems the maximum value of $p$ is usually of order $\mathrm{p} \ll \mathrm{n}$. In comparison, core storage required for the complete system matrix is $\left(n^{2}+2 n p+p^{2}\right)$ storage locations.

Use of minimum storage requirements and variation in $p$ throughout the loading history suggest consideration of alternate solution techniques for the set of simultaneous linear equations. 


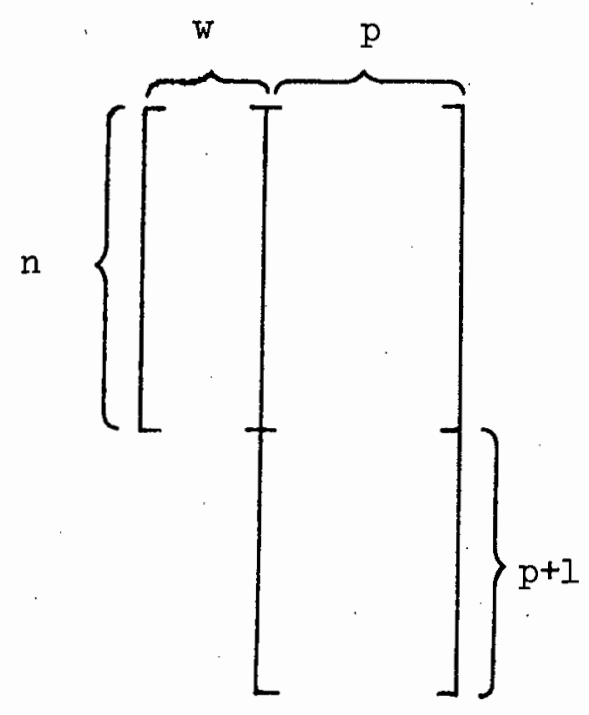

Figure 5.1 Minimum core storage requirements for system matrix [ $\left.\mathrm{K}^{*}\right]$

\subsection{Partitioning and Triangular Decomposition}

Rewriting equation (5.1) concisely as

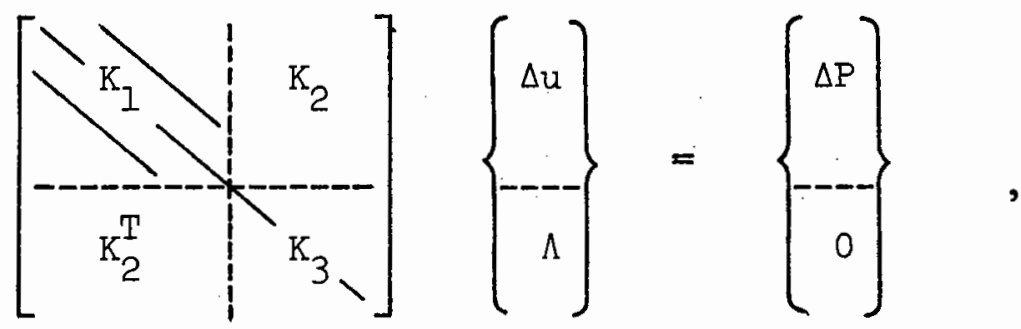

and eliminating displacement increments from the second set of the partitioned equations gives

$$
\left[\mathrm{K}_{3}-\mathrm{K}_{2}^{\mathrm{T}} \mathrm{K}_{1}^{-1} \mathrm{~K}_{2}\right]\{\Lambda\}=\left\{-\mathrm{K}_{2}^{\mathrm{T}} \mathrm{K}_{1}^{-1} \Delta \mathrm{P}\right\}
$$

where we note that the inverse of the elastic system matrix [ $\left.\mathrm{K}_{1}\right]$ is required. This appears attractive as $\left[K_{1}\right]$ is unchanged for each load increment and therefore need be inverted once only for each analysis problem. However the inverse of a symmetric banded matrix is symmetric 
but not banded and therefore approximately $\frac{1}{2} \mathrm{n}^{2}$ storage locations would be required to store a lower or upper triangle. Instead, using elementary row operations $\left[K_{1}\right]$ is decomposed into the product of two triangular matrices, each retaining the half band-width of $\left[\mathrm{K}_{l}\right]$. Further, since $\left[K_{1}\right]$ is symmetric these triangular matrices are transposes of one another, and thus we may write

$$
\left[\mathrm{K}_{1}\right]=\left[\mathrm{U}^{\mathrm{T}}\right][\mathrm{U}] \text {, }
$$

where [U] is an $\mathrm{n} \times \mathrm{n}$ upper triangular matrix with band-width w.

Entries in the band of [U] are determined from $\left[K_{1}\right]$ in situ, occupying the storage space originally allocated to the half band of $\left[K_{l}\right]$.

Substituting for $\left[K_{1}\right]$ in equation (5.3) gives

$$
\left[K_{3}-K_{2}^{T}\left(U^{T} U\right)^{-1} K_{2}\right]\{\Lambda\}=\left\{-K_{2}^{T}\left(U^{T} U\right)^{-1} \Delta P\right\}
$$

and writing $[\mathrm{V}]=\left[\left(\mathrm{U}^{\mathrm{T}}\right)^{-1} \mathrm{~K}_{2}\right]$ this becomes

$$
\left[K_{3}-V^{T} V\right]\{\Lambda\}=\left\{-V^{T}\left(U^{T}\right)^{-1} \Delta P\right\}
$$

In evaluating [V] the ith column is calculated from

$$
\{v\}_{i}=\left[U^{T}\right]^{-1}\left\{K_{2}\right\}_{i},
$$

or more conveniently

$$
\left.\left[U^{\mathrm{T}}\right]_{i}\right\}_{i}=\left\{\mathrm{K}_{2}\right\}_{i}
$$


where the triangularity of $\left[\mathrm{U}^{\mathrm{T}}\right]$ reduces evaluation of $\{\mathrm{V}\}_{i}$ to a forward substitution process only. The columns of [V] are calculated in situ in $\left[\mathrm{K}_{2}\right]$ and thus no additional storage is required. Once [V] has been determined the $p$ by $p$ matrix $\left[\bar{K}_{3}\right]$ can be formed, where

$$
\left[\bar{K}_{3}\right]=\left[\mathrm{K}_{3}-\mathrm{V}^{\mathrm{T}} \mathrm{V}\right]
$$

and is stored in the $\mathrm{p}$ by $\mathrm{p}$ work area described previously.

Using forward substitution on $\left[\mathrm{U}^{\mathrm{T}}\right]$ and pre-multiplying by $\left[\mathrm{V}^{\mathrm{T}}\right]$, the right-hand side of equation (5.3) is evaluated as

$$
\{\overline{\mathrm{P}}\}=\left\{-\mathrm{V}^{\mathrm{T}}\left(\mathrm{U}^{\mathrm{T}}\right)^{-1} \Delta \mathrm{P}\right\}
$$

and hence equation (5.3) reduces to

$$
\left[\overline{\mathrm{K}}_{3}\right]\{\Lambda\}=\{\overline{\mathrm{P}}\}
$$

where the matrix $\left[\bar{K}_{3}\right]$ is symmetric. Hence the p plastic multipliers are obtained using Gauss elimination and back substitution.

The plastic submatrix $\left[\mathrm{K}_{2}\right]$ which was destroyed in the formation of $[V]$ is now retrieved from peripheral storage (where it was retained in the event of iteration within a load increment). Writing the first $\mathrm{n}$ equations of $(5.2)$ as

$$
\left[U^{T} U\right]\{\Delta u\}=\left\{\Delta P-K_{2} \Lambda\right\}
$$

the right hand side is evaluated explicitly as $\left\{\mathrm{P}^{*}\right\}$. Introducing a new vector $\{v\}$ such that 


$$
\{v\}=[U]\{\Delta u\},
$$

equation (5.12) becomes

$$
\left[\mathrm{U}^{\mathrm{T}}\right]\{\mathrm{v}\}=\left\{\mathrm{P}^{*}\right\},
$$

from which $\{v\}$ is determined by forward substitution. Displacement increments $\{\Delta \mathrm{u}\}$ are then obtained through back substitution in equation (5.13).

The $\left[\mathrm{U}^{\mathrm{T}}\right][\mathrm{U}]$ decomposition of $\left[\mathrm{K}_{1}\right]$ is performed only once in the analysis procedure. Since the subsequent $(p+3)$ substitutions on $[\mathrm{U}]\left(\right.$ or $\left.\left[\mathrm{U}^{\mathrm{T}}\right]\right)$ do not require much computational effort, the solution technique is efficient when $p$ is small - that is when loading is such that only a small part of the body is plastic. Computational effort increases significantly as $p$ increases because in addition to forward and back substitutions on $[\mathrm{U}]$ or $\left[\mathrm{U}^{\mathrm{T}}\right]$, the plastic multipliers are determined from equation (5.11). This entails evaluation of $\left[\bar{K}_{3}\right]$ and $\left\{P^{*}\right\}$, and Gauss reduction of $p$ simultaneous linear equations.

\subsection{Gauss Elimination}

The second technique considered involves Gauss elimination operating on both elastic and plastic submatrices of $\left[\mathrm{K}^{*}\right]$ for each solution. Again referring to the matrices of equation (5.2) only the half band of $\left[K_{1}\right]$ and elements of $\left[K_{2}\right]$ and $\left[K_{3}\right]$ on and above the leading diagonal are stored as depicted in Figure (5.1). A modified Gauss elimination procedure is employed to operate on the distorted matrix and load increment vector, reducing [ $\left.\mathrm{K}^{*}\right]$ to an upper triangular 
form. Plastic multipliers and displacement increments are then obtained by back substitution.

Note that this solution procedure entails Gauss elimination of the constant submatrix $\left[\mathrm{K}_{1}\right]$ for each solution, and is therefore inefficient when $p$ is small.

\subsection{Relative Computational Efficiency}

In the preceding sections two different solution techniques have been proposed for solution of the set of simultaneous linear equations (4.34). The computational effort required by each method can be approximately assessed.

Since zero entries in the system matrix permit a reduction in the arithmetic required to produce a solution, the density, or number of non-zero entries in a matrix, affects the computation time. Employing a Judicious nodal numbering scheme, for most assemblages of finite elements the elastic stiffness matrix $\left[K_{l}\right]$ is tightly banded. For convenience let us here assume that the band-width is fully populated with non-zero entries. Density of the $\left[\mathrm{K}_{2}\right]$ submatrix is known: in the case of triangular constant strain finite elements there are only six non-zero entries per column, while for cubic quadrilateral isoparametric elements there are twenty-four non-zero entries per column. However, the number of arithmetic operations performed on $\left[\mathrm{K}_{2}\right]$ during either solution procedure is dependent on the position of the first non-zero entry encountered in each column of $\left[\mathrm{K}_{2}\right]$. This, in turn, is dependent on the nodal numbering scheme and node numbers corresponding to plastic elements. Although overall computational effort expended in producing a solution by either method is therefore problem dependent, an 'average' assessment can be made. 
Again consider the solution of a system of equations (5.2) in $\mathrm{n}$ displacement increment variables and at a stage in the loading when there are $p$ plastic multiplier variables. As before let the half band-width of $\left[\mathrm{K}_{1}\right]$ be w. Assume that the $\left[\mathrm{U}^{\mathrm{T}}\right][\mathrm{U}]$ decomposition of $\left[\mathrm{K}_{1}\right]$ has already been performed. Treating a computer subtraction operation as equivalent to one of addition, and considering only significant third-order terms in $n$, $w$ and $p$, we find that the technique of partitioning and triangular decomposition requires approximately $\left(n p^{2}+2 n w p\right)$ additions and $\left(n p^{2}+n w p\right)$ multiplications to produce a solution. The second technique (Gauss elimination of $\left[\mathrm{K}^{*}\right]$ ) consists of approximately $\left(\frac{1}{4} n p^{2}+n w p+2 n w^{2}\right)$ additions and $\left(\frac{1}{4} n p^{2}+n w p+n w^{2}\right)$ multiplications. Since an addition operation takes approximately three quarters of the execution time required to perform a multiplication operation, the number of computations for each technique may be written in terms of addition operations only as $\left(2,3 n p^{2}+3,3 n w p\right)$ and $\left(0,6 n p^{2}+2,3 n w p\right.$ $+3,3 \mathrm{nw}^{2}$ ) respectively. Equating these numbers of addition operations, the two methods have approximately the same efficiency when

$$
1,7 p^{2}+w p-3,3 w^{2}=0,
$$

i.e. when $p=1,1 w$. (From equation (5.15) the comparison of computational effort for each of the two methods is independent of $n$ ). In summary, we deduce that the technique of partitioning and triangular decomposition is more efficient than Gauss elimination of $\left[\mathrm{K}^{*}\right]$ until the number of plastic multiplier variables is approximately equal to the half band-width of $\left[\mathrm{K}_{1}\right]$. If $\mathrm{p}$ increases beyond this we expect the latter technique to become more efficient. 
Figures $(5.2 a)-(5.2 d)$ show the execution time ${ }^{\dagger}$ required per solution plotted against $\mathrm{p}$, for four analyses of continua, each with monotonic increase of load. The nodal numbering of each problem was such that the band of $\left[K_{l}\right]$ was densely populated, having few zero terms. In Figures (5.2)(a) and (b.) the analyses employed isoparametric finite elements, while those of Figures $(5.2)(c)$ and $(d)$ are results of constant strain finite element analyses. Note in (c) and (d) the curves intersect at a value of $p$ slightly greater than $p=1,1 \mathrm{w}$.

As noted previously the number of arithmetic operations performed on the $\left[\mathrm{K}_{2}\right]$ submatrix of equation (5.2) is dependent on two factors. Firstly, since a string of zero entries in a coliumn of the submatrix permits a reduction in computation, the position of the first non-zero entry encountered during solution affects the total computation time. The second factor (which directly influences the first) is the overall density of $\left[\mathrm{K}_{2}\right]$. In the quantitative assessment of numbers of arithmetic operations resulting in the computational equivalence of the two solution methods at $p=1,1 \mathrm{w}$, average density and random locations of non-zero entries in $\left[\mathrm{K}_{2}\right]$ were assumed. In (c) and $(d)$ the additional sparseness of $\left[K_{2}\right]$ for constant strain element analyses (six non-zero entries per column compared with twenty-four) causes the partitioning technique to remain more efficient for values of $\mathrm{p}$ slightly greater than $\mathrm{p}=1,1 \mathrm{w}$.

In the computer programs developed by the writer to implement the application of the minimum principle to elasto-plastic analysis of continua, both solution techniques are available. Since relative computational efficiencies of the two techniques are problem dependent with variation related to node numbers of plastic elements, the computer 

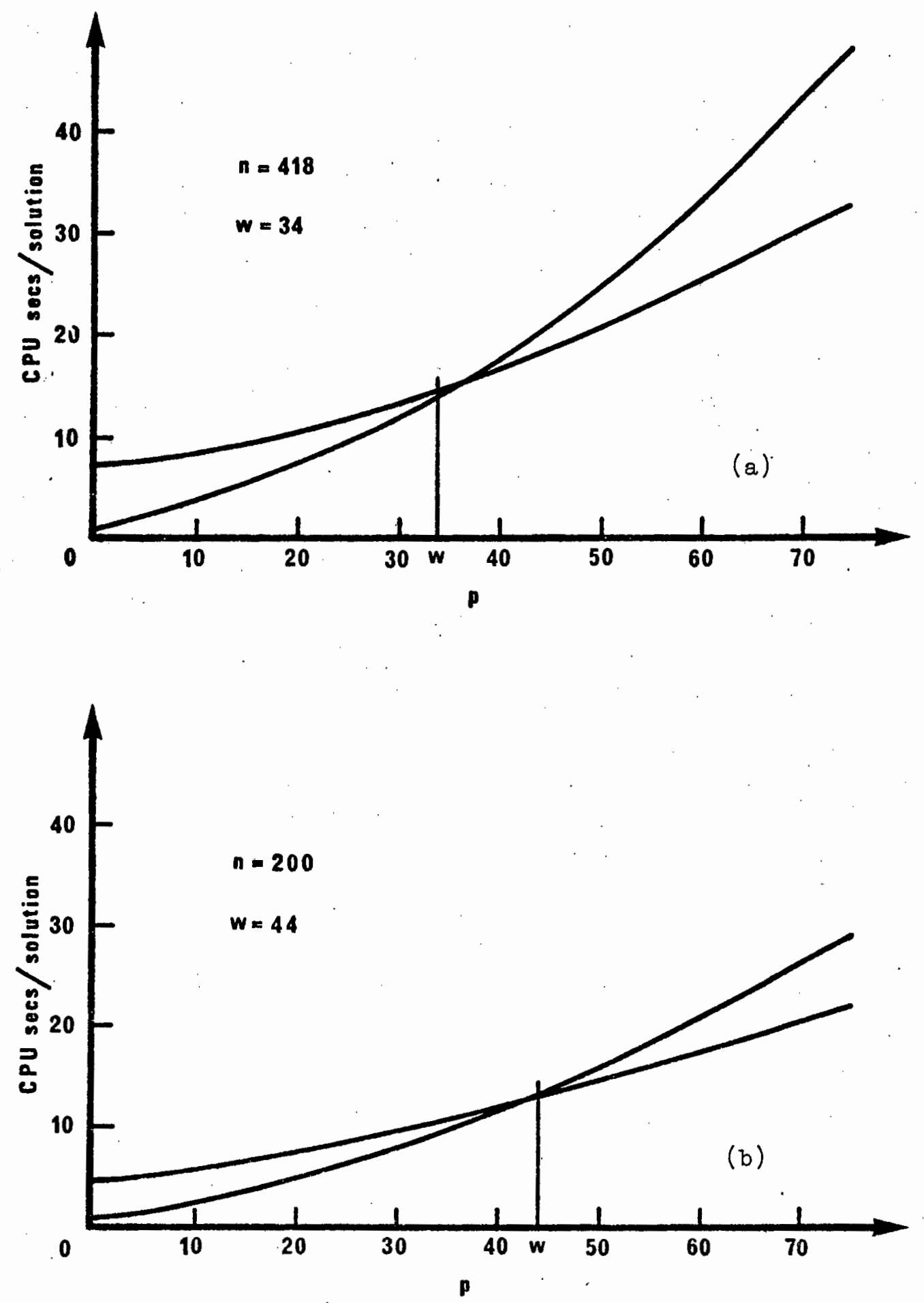

Figure 5.2. CPU time/solution versus number of plastic multipliers $p$ 

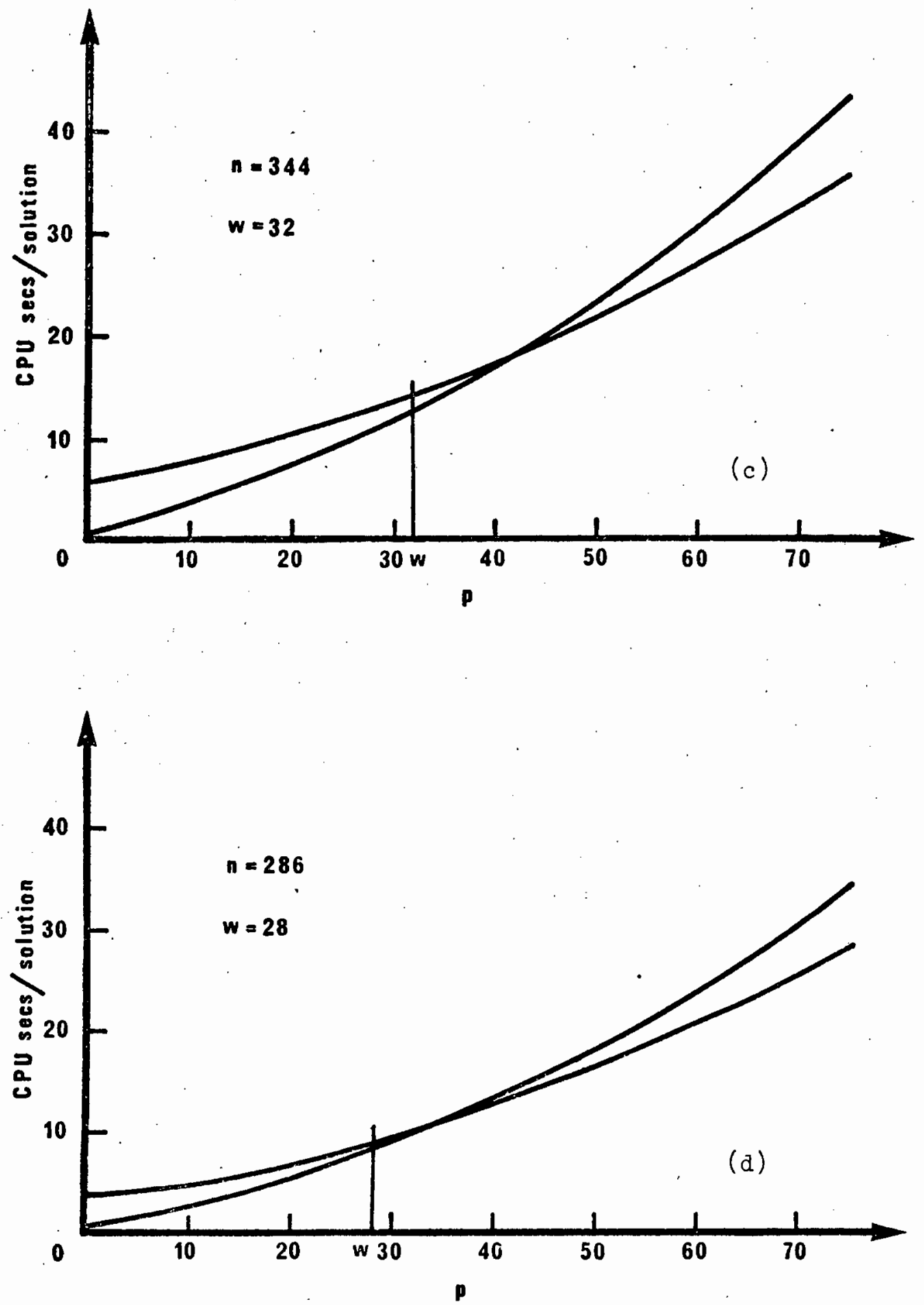

Figure 5.2 CPU time/solution versus number of plastic multipliers $p$ 
programs select the solution method arbitrarily on the basis of $\mathrm{p}<\mathrm{w}$ or $\mathrm{p} \geq \mathrm{w}$ : if $\mathrm{p}<\mathrm{w}$ partitioning and triangular decompsition is used, and if $p \geq w$ Gauss reduction of $\left[K^{*}\right]$ is used.

\subsection{Uniqueness of Solution in Elastic-Perfectly Plastic Case}

The functional $\overline{\mathrm{W}}_{\mathrm{p}}^{0}$ of equation (3.20) subject to constraints (3.21) represents in rate form, the strain energy of the body. It follows therefore, that in the numerical application of the minimum principle the system matrix $\left[\mathrm{K}^{*}\right]$ is positive definite unless flow occurs in the elastic-perfectly plastic case.

Consider an assemblage of elastic-perfectly plastic constant strain finite elements at the point of flow. Let there be say, $p$ plastic elements at this stage. Ordering the plastic elements and taking the weighted plastic strain rate components in turn leads to a vector $\left\{\dot{\delta}^{p}\right\}$. At flow the plastic strain rate is the total strain rate, hence

$$
\left\{\dot{\delta}^{p}\right\}=[\bar{B}]\{\dot{u}\}
$$

where $\{\dot{u}\}$ is the vector of nodal velocities and $[\bar{B}]$ is a condensed system deformation matrix containing only rows of [B] corresponding to elements of $\left\{\dot{\delta}^{p}\right\}$. From equation (4.23)

$$
\left\{\dot{\delta}^{p}\right\}=[\bar{N}]\{\Lambda\}
$$

where $[\bar{N}]$ is a condensed gradient matrix containing only columns of [N] corresponding to non-zero elements of $\{\Lambda\}$. Combining equations $(5.16)$ and $(5.17)$ gives 


$$
[\bar{B}]\{\dot{u}\}=[\bar{N}]\{\Lambda\} .
$$

Now defining $[\bar{D}]$ as a block diagonal matrix containing element elasticity matrices $\frac{l}{V},\left[D^{\prime}\right]$ for the ordered plastic elements, the system of equations at flow is

$$
\left[B^{\mathrm{T}} \mathrm{DB}\right]\{\dot{\mathrm{u}}\}-\left[\overline{\mathrm{B}}^{\mathrm{T}} \overline{\mathrm{D}} \overline{\mathrm{N}}\right]\{\Lambda\}=\{\dot{\mathrm{P}}\}
$$

and $-\left[\overline{\mathrm{N}}^{\mathrm{T}} \overline{\mathrm{D}} \overline{\mathrm{B}}\right]\{\dot{\mathrm{u}}\}+\left[\overline{\mathrm{N}}^{\mathrm{T}} \overline{\mathrm{D}} \overline{\mathrm{N}}\right]\{\Lambda\}=\{0\}$.

Substituting for $[\bar{B}]\{\dot{u}\}$ from equation (5.18) in the partitioned equations (5.20) gives

$$
\left[-\overline{\mathrm{N}}^{\mathrm{T}} \overline{\mathrm{D}} \overline{\mathrm{N}}+\overline{\mathrm{N}}^{\mathrm{T}} \overline{\mathrm{D}} \overline{\mathrm{N}}\right]\{\Lambda\}=\{0\},
$$

that is

$$
[0]\{\Lambda\}=\{0\},
$$

indicating that the system matrix $\left[\mathrm{K}^{*}\right]$ is singular at the load corresponding to incipient plastic flow. The results (5.21) was obtained by considering an assemblage of constant strain finite elements. Following a similar argument for elastic-perfectly plastic isoparametric finite elements leads to an identical result.

In the numerical analysis of test problems the value of the determinant was found to be unreliable in establishing the limit load. This is attributed to two causes. Firstly, the analysis procedure is incremental and piecewise-linear in the sense that elastic and plastic regions of the body are treated as unchanged during a load increment of finite magnitude. New elastic-plastic boundaries are then established and the next load increment applied. In this way the limit load of the 
assemblage is not approached asymptotically, but in increments of finite magnitude. The second cause is attributed to numerical error accumulation during successive incremental solutions. Since the determinant is evaluated as the product of terms on the leading diagonal of the triangulated system matrix, possible ill-conditioning of the system of equations as the limit load is approached makes the value of the determinant unreliable. At this stage the minimization algorithm does not always: converge, but may iterate repetitively interchanging the same groups of loading and unloading plastic elements in successive cycles.

In elastic-perfectly plastic problems a good estimate of limit load is obtained by determining the load at which load increment magnitudes become negligibly small for several successive load increments.

\subsection{Load Increment Magnitude}

In the finite element application of the minimum principle, the maximum magnitude of any load increment is determined by the smallest increment of load which causes an element of $v_{e}$ (where $\phi<0$ ), to enter $\mathrm{V}_{\mathrm{p}}$ (where $\left.\phi=0\right)$.

Consider an arbitrary stage in the loading program of an assemblage of elements. Current totals of stress, elastic strain, plastic strain, load and displacement are known. For the next load increment the system matrix $\left[\mathrm{K}^{*}\right]$ is assembled. In determining a solution for this increment the load increment vector $\{\Delta \mathrm{P}\}$ need only reflect relative magnitudes of load components. After a solution is determined satisfying the constraints, we have increments of stress, elastic strain, plastic strain and displacement corresponding to the arbitrary magnitude of applied load increment $\{\Delta \mathrm{P}\}$. 
To determine the correct load increment magnitude we consider each elastic element (for the isoparametric case each elastic integration point) and determine a factor $\rho$ such that $\phi\left(\sigma_{i j}+\rho \Delta \sigma_{i j}, \varepsilon_{i j}^{p}\right)=0$, where $\sigma_{i j}$ and $\varepsilon_{i j}^{p}$ are current totals of stress and plastic strain at completion of the previous load increment, and $\Delta \sigma_{i j}$ is the stress increment corresponding to the current load increment $\{\Delta \mathrm{P}\}$.

From the expression for the von Mises yield function (equation 2.18) we have

$$
\frac{1}{3}\left\{\left(\bar{\sigma}_{i j}+\rho \Delta \sigma_{i j}\right)\left(\bar{\sigma}_{i j}+\rho \Delta \sigma_{i j}\right)-\frac{1}{3}\left(\bar{\sigma}_{k k}+\rho \Delta \sigma_{h h}\right)^{2}\right\}-\frac{2}{9} \sigma_{0}^{2}=0
$$

To solve for $\rho$ define a quantity $\phi^{*}$ as

$$
\phi^{*}(\alpha, \beta)=\frac{1}{3}\left\{\alpha_{i j} \beta_{i j}-\frac{1}{3} \alpha_{k k} \beta_{\ell \ell}\right\}
$$

and expand equation (5.22) to give

$$
\rho^{2} \phi^{*}(\Delta \sigma, \Delta \sigma)+2 \rho \phi^{*}(\bar{\sigma}, \Delta \sigma)+\phi *(\bar{\sigma}, \bar{\sigma})-\frac{2}{9} \sigma_{0}^{2}=0
$$

Since $\phi^{*}(\bar{\sigma}, \Delta \sigma)$ and $\phi^{*}(\bar{\sigma}, \bar{\sigma})$ are non-negative and $\phi^{*}(\Delta \sigma, \Delta \sigma)$ is always greater than zero, the positive root of equation (5.24) is given by

$$
\rho=\frac{-\phi^{*}(\bar{\sigma}, \Delta \sigma)+\sqrt{\left\{\phi^{*}(\bar{\sigma}, \Delta \sigma)\right\}^{2}-\phi^{*}(\Delta \sigma, \Delta \sigma)\left\{\phi^{*}(\bar{\sigma}, \bar{\sigma})-\frac{2}{9} \sigma_{0}^{2}\right\}}}{\phi^{*}(\Delta \sigma, \Delta \sigma)}
$$


A value of $\rho$ is calculated for each element of $V_{e}$ and the smallest, $\rho_{\text {min }}$, identified. All increment quantities for the current solution are scaled by this factor $\rho_{\min }$ and added to current totals to give the new state of the assemblage. The element of $\mathrm{V}_{e}$ associated with $\rho_{\min }$ is included in $\mathrm{v}_{\mathrm{p}}$ for the next load increment, as its stress point now lies in the yield surface. As noted previously, fortuitously there may be more than one element of $\mathrm{V}_{e}$ associated with $\rho_{\min }$, within the bounds of computational accuracy.

\subsection{Determination of $\mathrm{V}_{\mathrm{p}}$}

Following the procedure described in the previous section for determining load increment magnitudes leads to a large number of load increments, with actual magnitudes of increments becoming very small as large regions of the body become plastic. So as to decrease overall computation time elements of $\mathrm{v}_{e}$ for which $\phi$ is close to zero are included in $V_{p}$

At an arbitrary stage we wish to determine elements of $\mathrm{V}_{\mathrm{p}}$ for evaluation of the current system matrix [K*]. For the previous load increment $\rho_{\min }$ was determined and all increment quantities scaled by this factor. Hence, current totals of stress, strain, load and displacement were established. Considering each element of the assemblage in turn, (for the isoparametric case each integration point), the von Mises equivalent stress $\sigma_{\mathrm{eq}}$ is evaluated, where

$$
\sigma_{e q}=\sqrt{\phi\left(\bar{\sigma}_{i j}\right)+\sigma_{o}^{2}} \text {. }
$$


If $\sigma_{\text {eq }} \geq n \sigma_{\circ}$, where $n$ is a preassigned constant, the element (integration point) is included in $V_{p}$ for the start of the current load increment. After this increment stress corrections are made to ensure that $\phi=0$ for each element in $v_{p}$.

Yamada et al [15] follow a similar procedure and arbitrarily set $n=0,995$. However, for all the numerical examples investigated by the writer a value of $n=0,99$ was found to introduce negligible errors while decreasing computation time significantly. Unless otherwise stated or for the purpose of comparison of computation time, this value of $n$ is adopted for the numerical examples of Chapter 6 .

\subsection{Correction of Stresses in $\mathrm{V}_{\mathrm{p}}$ for Elastic-Perfectly Plastic Case}

In the elastic-perfectly plastic case stress points which do not unload undergo neutral loading, and for an infinitesimal stress increment the stress point moves in the yield surface. For a stress increment of finite magnitude the stress point moves along the tangent to the yield surface at that point, resulting in an inadmissible stress state for which $\phi>0$. The stress point is returned to the yield surface along a radial path by scaling stress components by a suitable factor.

In so doing equilibrium of the assemblage is violated. This could be accounted for by determining the nodal loads corresponding to the equilibrium violation and adding to the current total of load, continuing in an iterative manner until equilibrium is satisfied. However, for the numerical examples investigated by the writer this was deemed unnecessary as correction factors smaller than 0,998 did not occur. 
C H A P T E R 6

NUMERICAL EXAMPLES

\subsection{Introduction}

To illustrate application of the extended minimum principle representative results for analyses of some numerical examples are given below. Where possible comparisons are made with published results, thereby indicating orders of accuracy and efficiency. In particular results are given for a Vee-notched tension specimen in plane stress, plane strain or axial symmetry for either perfect plasticity or strain hardening. Comparisons are made with experimental and numerical results from the literature. The second numerical example is a deep cantilever in plane stress subjected to a parabolic shear distribution over the free end. No strain hardening is assumed, and upper bound values of the limit load are obtained for monotonically increasing load. Elaștoplastic loading and unloading are shown for cyclic loading. Results are compared with those of other numerical analyses. Finally, an axisymmetric pressure vessel-flush nozzle junction is analyzed under increasing internal pressure. Comparisons are made with experimental and numerical results.

\subsection{Vee-Notched Tension Specimen}

One of the earliest papers giving results of a numerical analysis of an elastic-plastic continuum is that of Marcal and King, (1967) [14]. A notched tension specimen was analyzed in plane stress, plane strain and axial symmetry, assuming an elastic-perfectly plastic von Mises 
material. This problem has subsequently been investigated by others such as Yamada, Yoshimura and Sakurai, (1968) [15], Zienkiewicz, Valliapan and King (1969) [11], and Anand and Shaw (1977) [30], each giving results for plane stress analyses only. Experimental results for such a specimen have been reported by Theokaris and Marketos (1963) [31], who used a technique of birefringent coating and polarized light to determine principal strain distributions for the elastic-perfectly plastic plane stress problem.

The geometry of the specimen is shown in Figure 6.2.1. The notch-depth to half-width ratio is 1 to 2 and notch angle $90^{\circ}$. For the present analysis mechanical properties of the material are $E=20000 \mathrm{~kg} / \mathrm{mm}^{2}$; $\nu=0,3 ; \quad \sigma_{0}=30 \mathrm{~kg} / \mathrm{mm}^{2}$, and for the case of hardening $E_{p}=650 \mathrm{~kg} / \mathrm{mm}^{2}$. From Figure 6.2.1 it may be seen that due to two way symmetry only one quadrant need be considered in the analysis. Loading consists of a uniformly distributed tensile load applied to the ends of the specimen. Using the computer programs listed in the appendix various analyses of the notched specimen were performed for monotonically increasing tensile loading. The first set comprises constant strain finite element analyses using 244 elements and 143 nodes. The element discretization of a quadrant is shown in Figure 6.2.2.

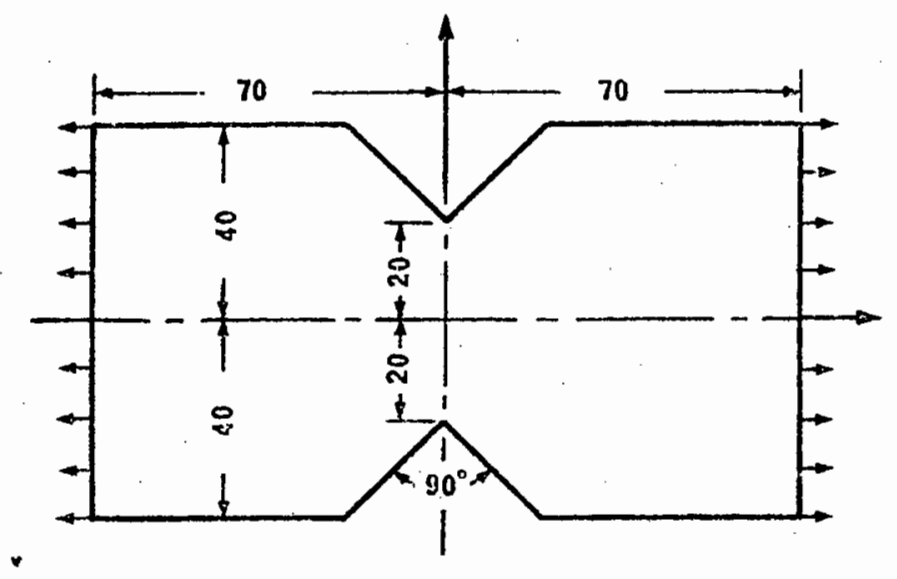

Figure 6.2.I Vee-notched tension specimen 


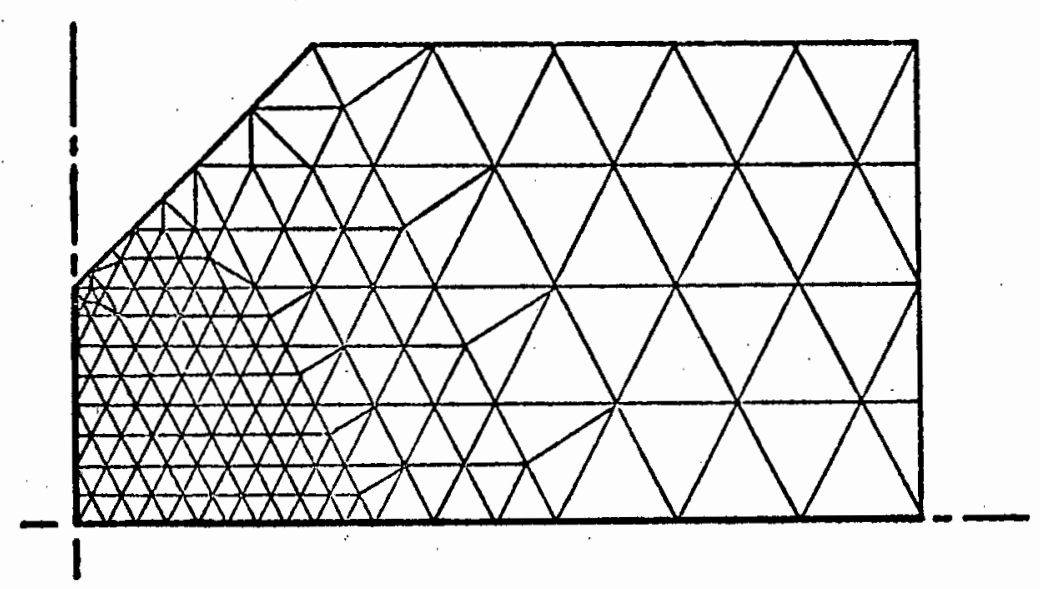

Figure 6.2.2 Constant strain finite element mesh for quadrant of vee-notched specimen

With no strain hardening, plastic elements at some representative stages of calculation are shown in Figure $6.2 .3,4$ and 5 respectively for the cases of plane stress, plane strain and axial symmetry. In these Figures load levels are given in dimensionless form as $\sigma_{m} / \sigma_{0}$ where $\sigma_{m}$ is the mean stress at the minimum section. The effect of orientation of triangular constant strain elements, previously observed by Yamada et al [15] and Anand and Shaw [30], is clearly evident. For the plane stress analysis the plastic enclaves of Figures $6.2 .6 \mathrm{~b}$ and $6.2 .6 \mathrm{c}$ were drawn by smoothing the jagged elasto-plastic boundaries of finite element analyses due to Yamada et al and the present method, respectively. Experimental results reported by Theokaris and Marketos [3I] are shown in Figure 6.2.6a. Although the results of the present analysis are in excellent agreement with those of Yamada et al, the experimental results show development of a continuous plastic region across the width of the specimen at a lower load than that indicated by the numerical analyses. This is attributed to the fact that Theokaris and Marketos give only a minimum value for the uniaxial yield stress $\sigma_{0}$, 

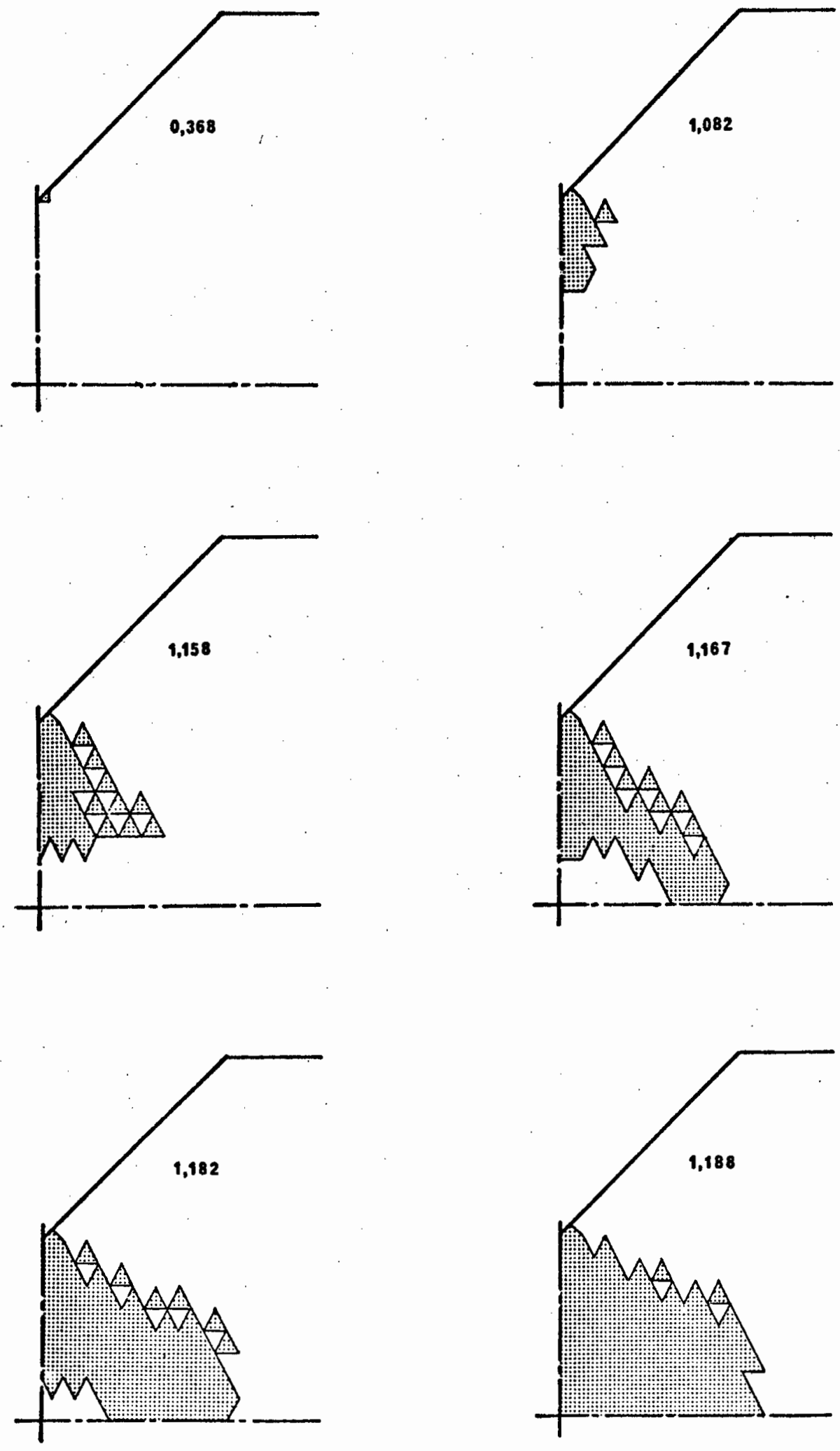

Figure 6.2.3 V-notched specimen, plane stress: constant strain finite element analysis, plastic elements at values of $\sigma_{\mathrm{m}} / \sigma_{0}$ 
79
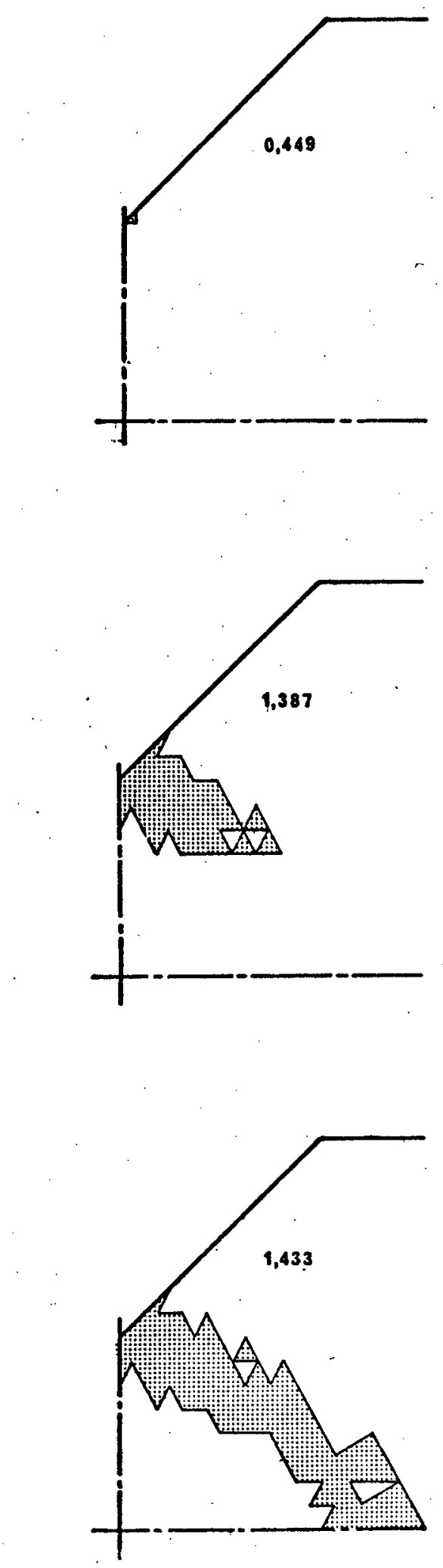
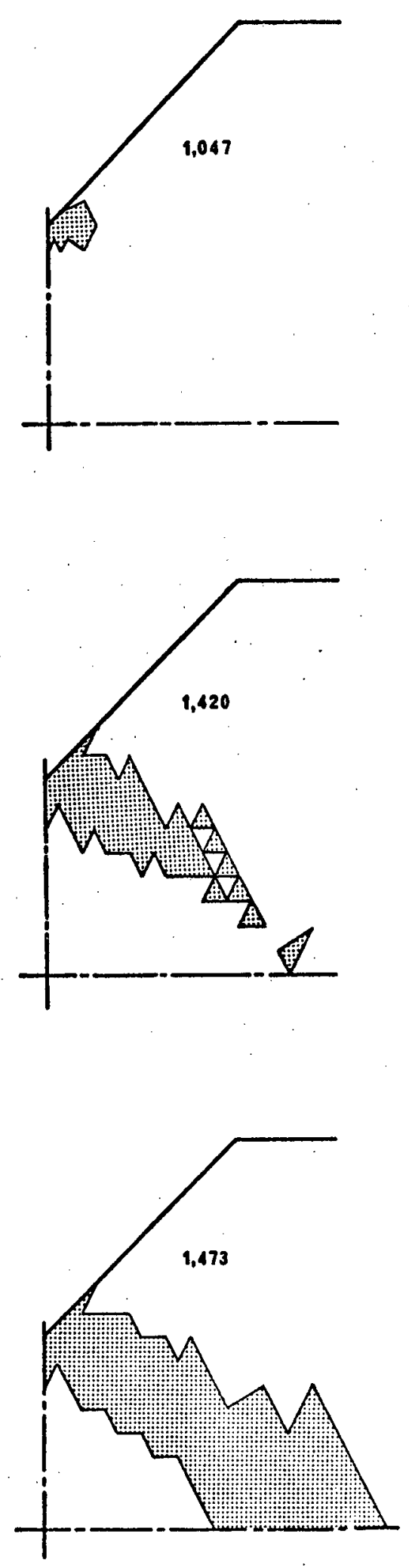

Figure 6.2 .4

$$
\begin{aligned}
& \text { V-notched specimen, plane strain: constant strain finite } \\
& \text { element analysis, plastic elements at values of } \sigma_{m} / \sigma_{0}
\end{aligned}
$$



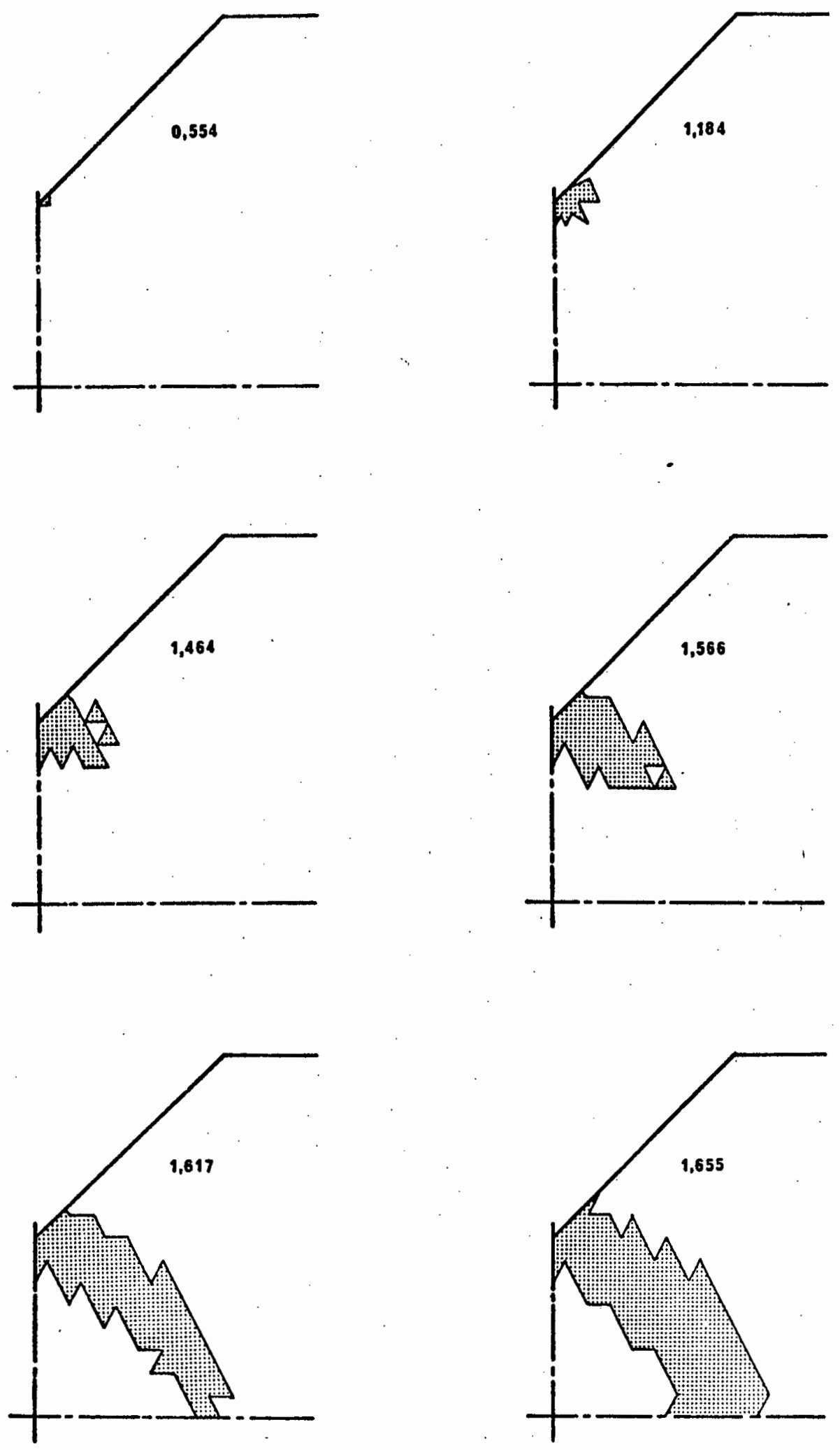

Figure 6.2.5 V-notched specimen, axial symmetry: constant strain finite element analysis, plastic elements at values of $\sigma_{m} / \sigma_{0}$ 


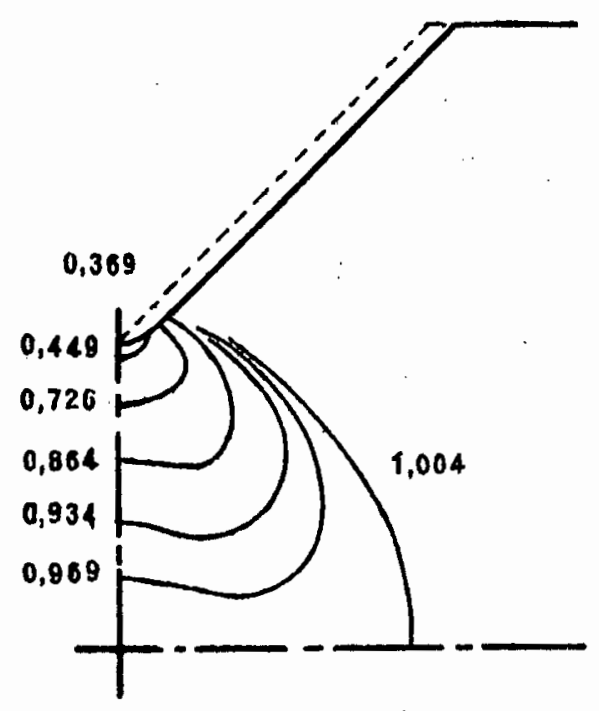

(a)

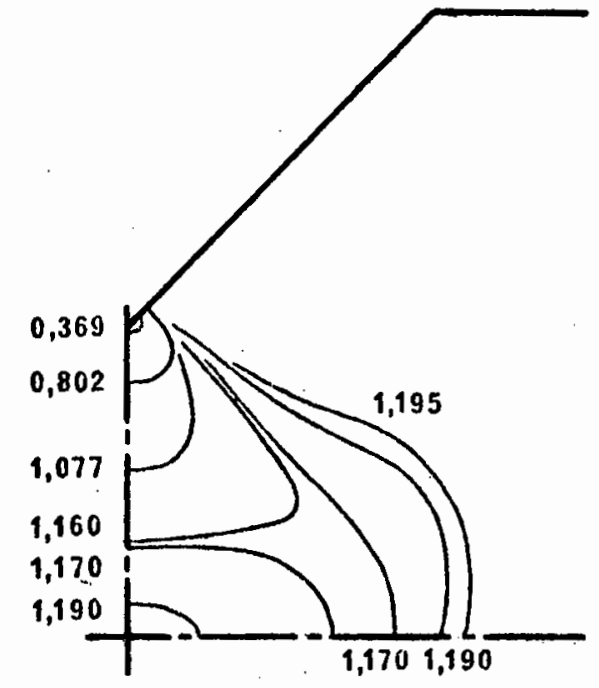

(b)

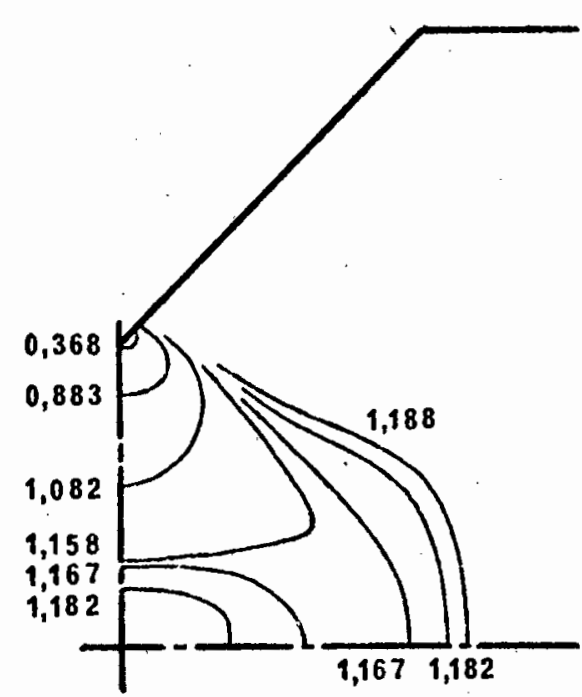

(c)

Figure 6.2.6 Vee-notched specimen, plane stress: plastic enclaves at values of $\sigma_{\mathrm{m}} / \sigma_{0}$; (a) Theokaris and Marketos, experimental; (b) Yamada et al; (c) Present analysis 
from which the enclaves at dimensionaless load levels in Figure 6.2.6a were drawn. If the true yield stress was slightly higher than this minimum value, then all plastic regions of the numerical analyses would be in reasonable agreement with those of the experimental results, with the exception of the value of initial yield load. For the numerical analyses the initial yield load is dependent on the finite element mesh in the region of the notch root, while for the experimental investigation the notch root radius was 0,18 of the notch depth. Thus comparisons of initial yield load are not meaningful.

Figure 6.2.7 shows in dimensionless form the maximum strain versus applied load for plane stress, plane strain and axisymmetric analyses using constant strain elements. The dashed curves in the Figure represent results of analyses assuming a kinematic hardening material with plastic modulus $E_{p}=650 \mathrm{~kg} / \mathrm{mm}^{2},\left(E_{p}=0,0325 \mathrm{E}\right)$. For all analyses the maximum longitudinal strain occurred in the element which had become plastic at the initial yield load. Again strain magnitudes are dependent on the finite element mesh at the notch root. The influence of work hardening is only significant at high loads.

Zienkiewicz, Valliapan and King (1969) [11] give results for the elasto-plastic analysis of a similar V-notched specimen under conditions of plane strain. No strain hardening was assumed. Mechanical properties are given as $E=7000 \mathrm{~kg} / \mathrm{mm}^{2} ; \quad \nu=0,2$ and $\sigma_{0}=24,3 \mathrm{~kg} / \mathrm{mm}^{2}$. Plastic enclaves due to Ziekiewicz et al are shown in Figure 6.2.8a for representative values of $\sigma_{\mathrm{m}} / \sigma_{0}$. Using the same constant strain finite element. mesh as used by Ziekiewicz et al ( 149 elements, 94 nodes), plastic enclaves resulting from an analysis using the present method are indicated in Figure 6.2.8b for similar load levels. Good agreement is obtained. 


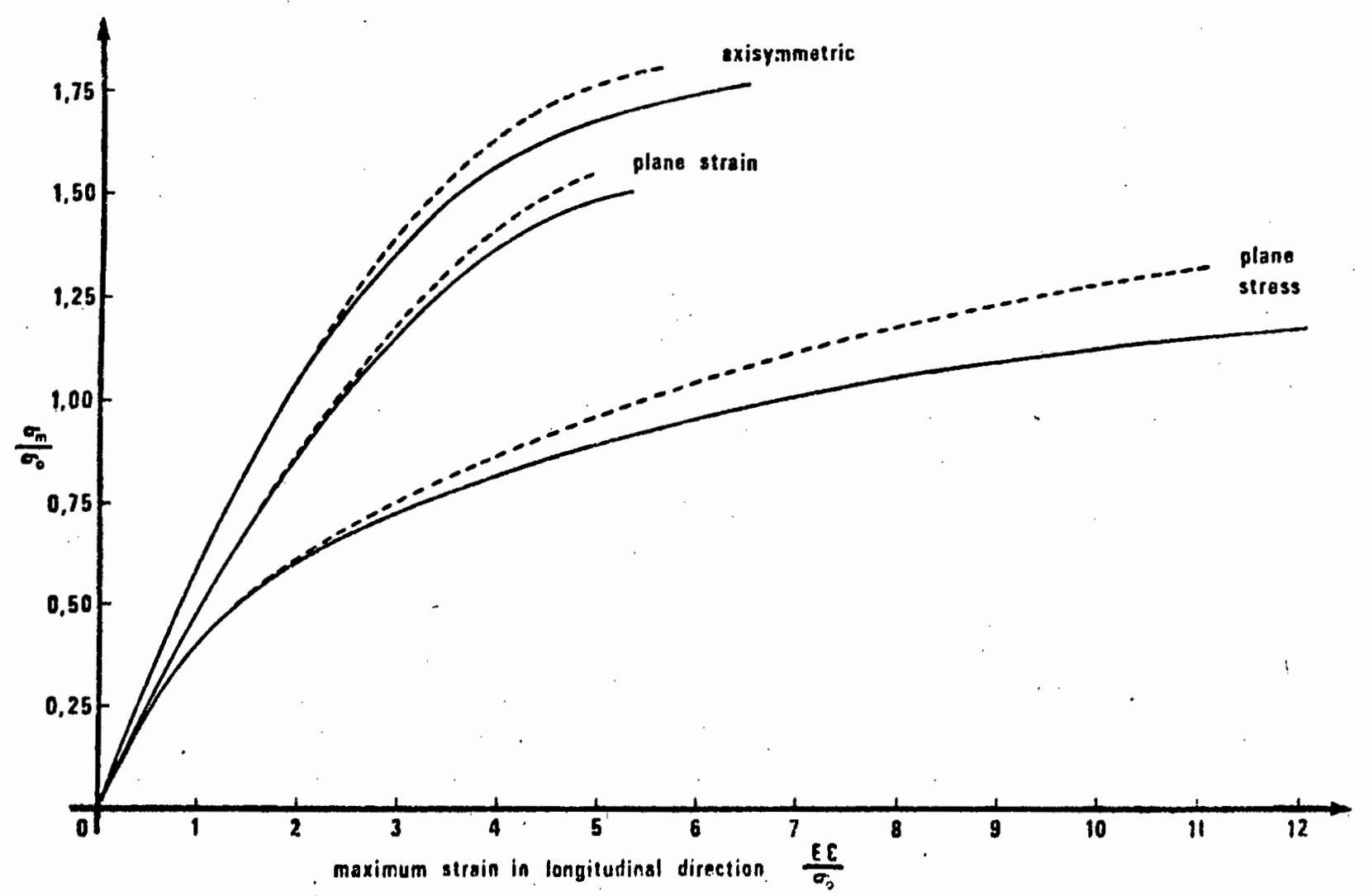

Figure 6.2.7 Load-strain curves for V-notched specimen, constant strain finite element analysis

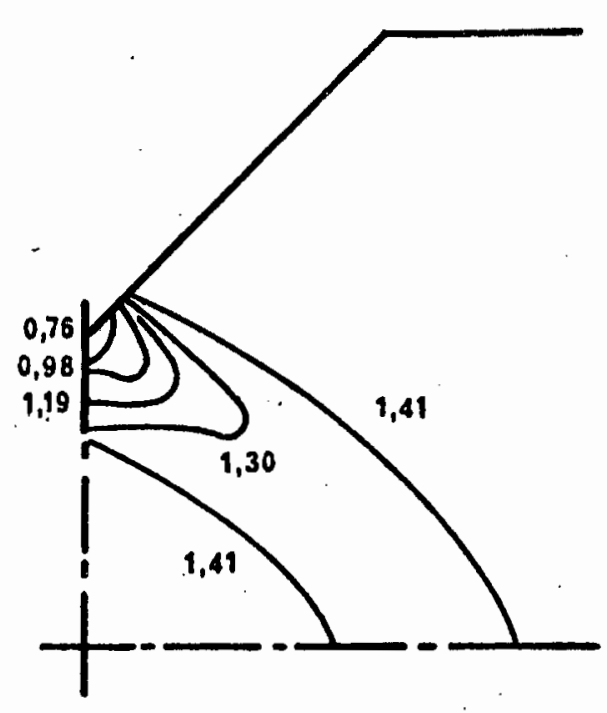

(a)

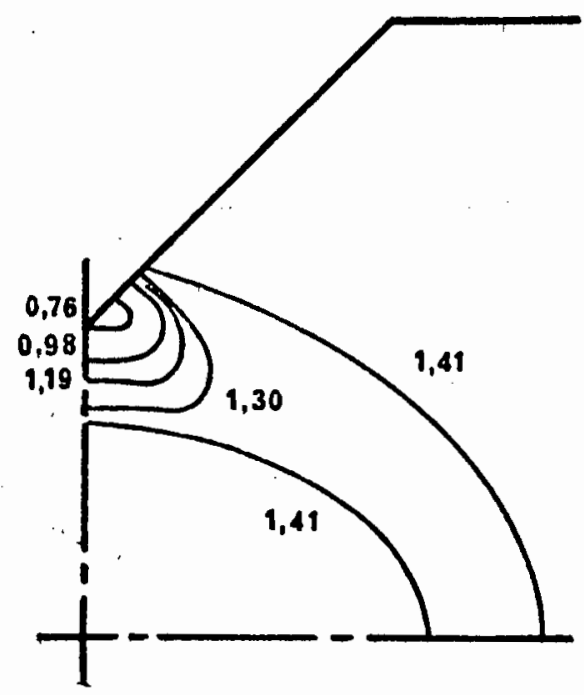

(b)

Figure 6.2.8 V-notched specimen, plane strain: plastic enclaves at values of $\sigma_{\dot{m}} / \sigma_{o} ;$ (a) Zienkiewicz et al;

(b) Present analysis 
The next set of results represent cubic quadrilateral isoparametric analyses of a similar notched tension specimen. A mesh of 15 elements and 100 nodes was used for the quadrant as shown in Figure 6.2.9. Nine integration points are indicated for each element, corresponding to third order Gauss integration. Because of the coarseness of the mesh, a plastic multiplier $\lambda$ was associated with each integration point (and not one 'average' multiplier for each element). With no strain hardening plastic integration points are shown in Figure 6.2.10, 11 , and 12 respectively for cases of plane stress, plane strain and axial symmetry. Even though a coarse finite element mesh was used these plastic regions are similar to those of constant strain finite element analysis shown in Figure 6.2.3, 4 and 5. Further, the curves of maximum longitudinal strain versus applied load shown in Figure 6.2.13 for the isoparametric results are in excellent agreement with those of Figure 6.2.7. In both these Figures solid lines correspond to elastic-perfectly plastic analyses while dashed curves result from a hardening material $E_{p}=0,0325 \mathrm{E}$. In the case of Figure 6.2.13 the maximum longitudinal strain occurs at the Gauss point closest to the notch root.

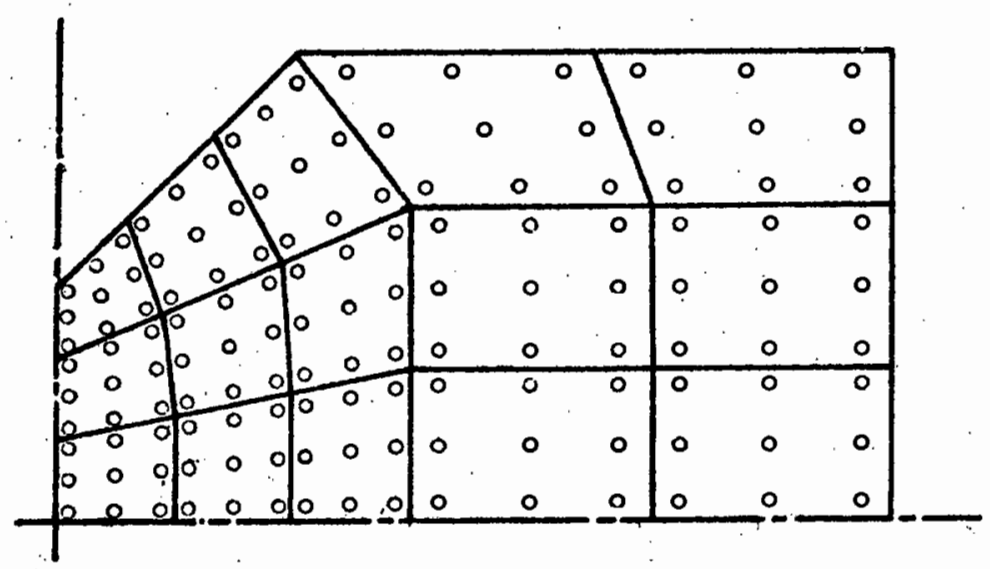

Figure 6.2.9 Cubic quadrilateral isoparametric finite element mesh for quadrant of $V$-notched specimen 

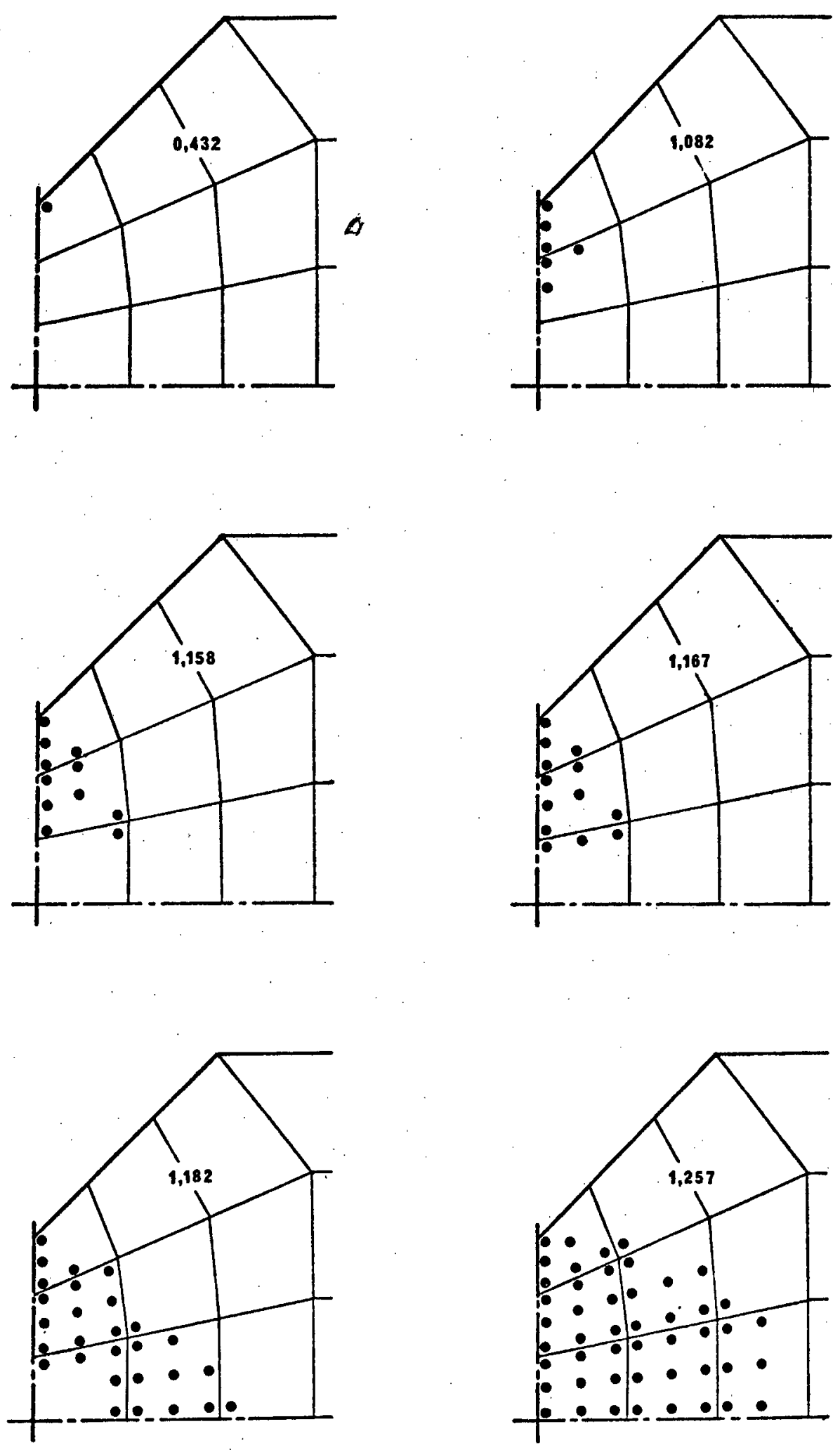

Figure 6.2.10 V-notched specimen, plane stress: isoparametric finite element analysis, plastic integration points at values of $\sigma_{\mathrm{m}} / \sigma_{\mathrm{o}}$ 

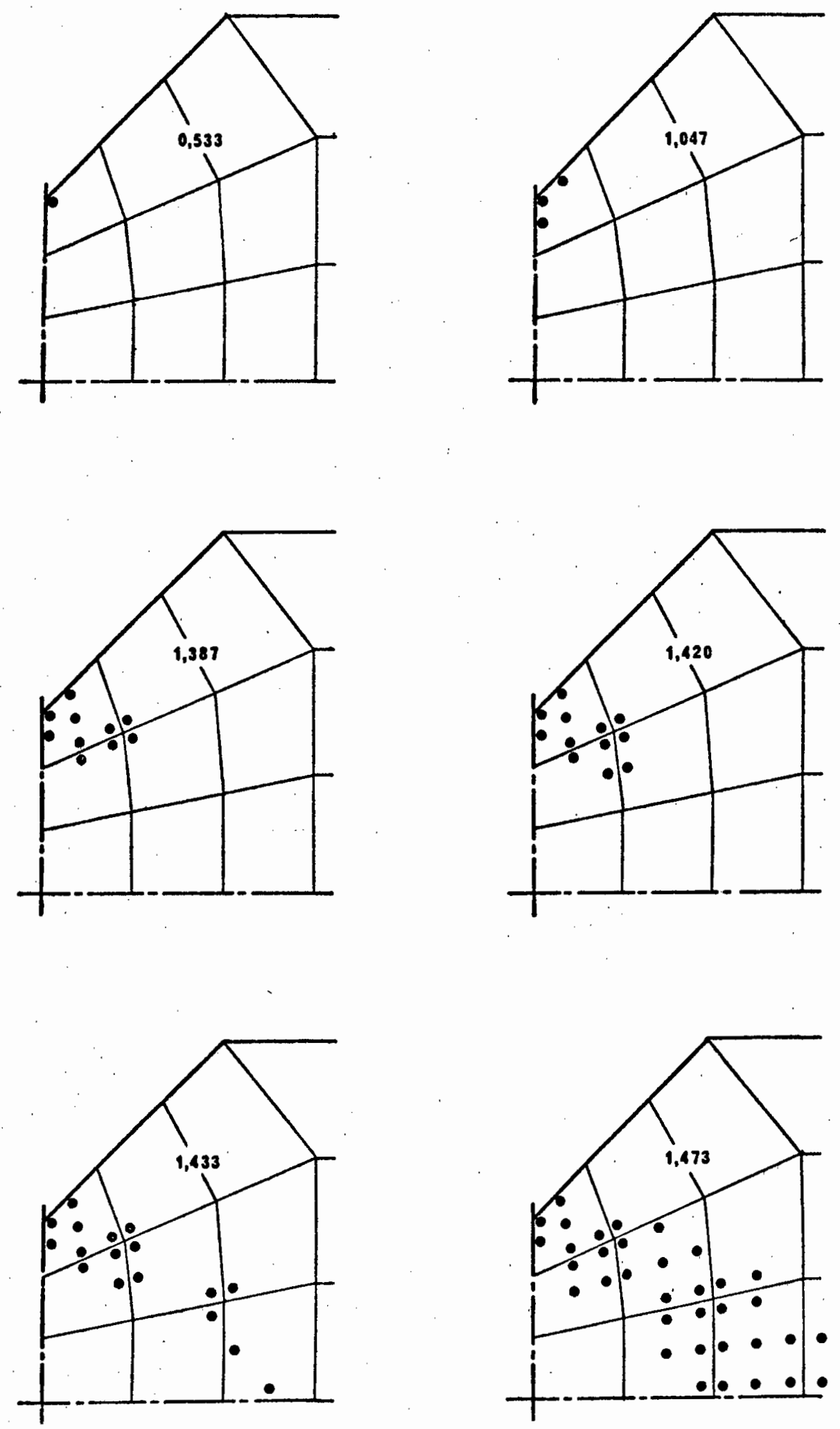

Figure 6.2.11 V-notched specimen, plane strain: isoparametric finite element analysis, plastic integration points at values $\sigma_{m} / \sigma_{0}$ 

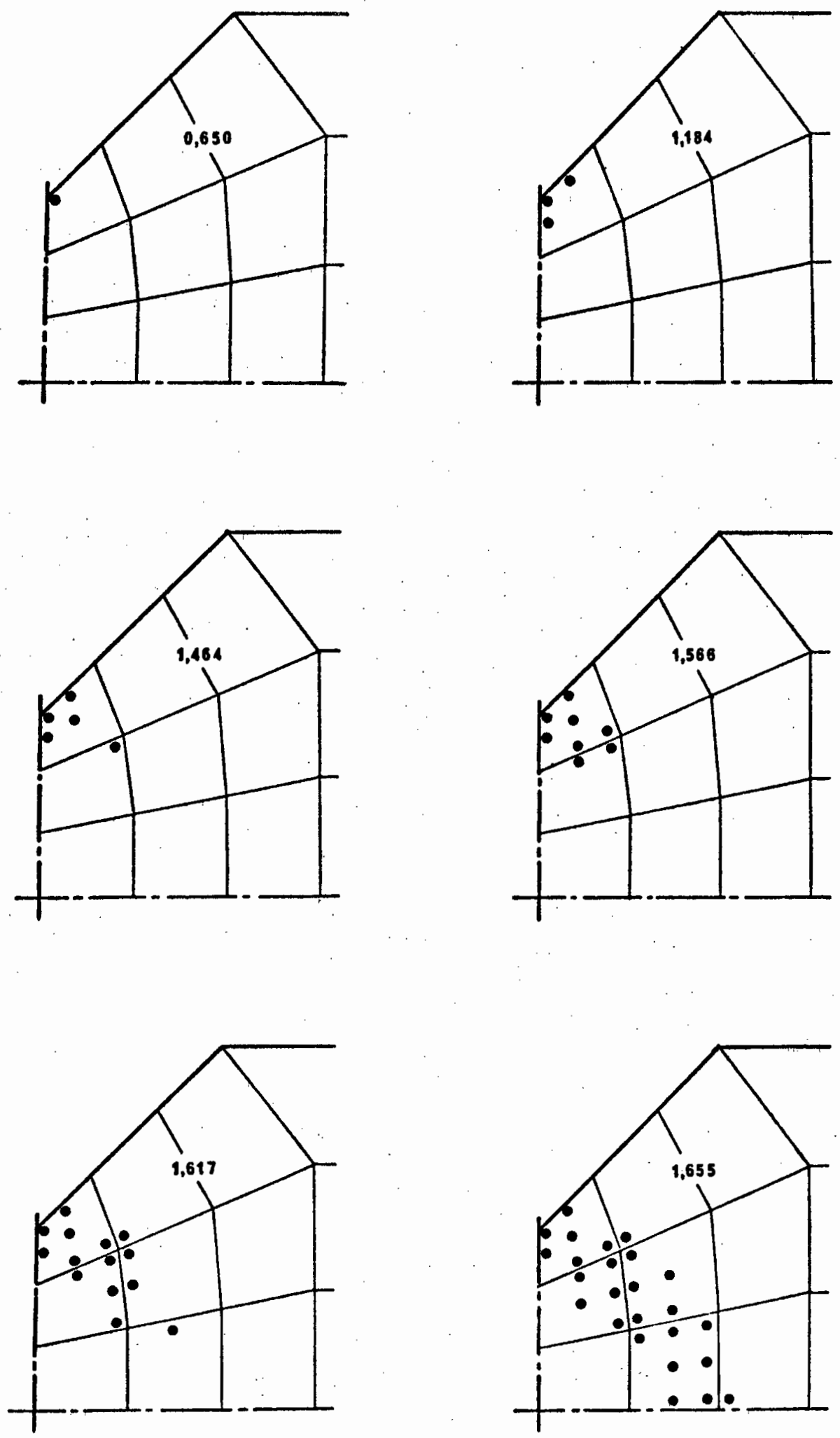

Figure 6.2.12 V-notched specimen, axial symmetry: isoparametric finite element analysis, plastic integration points at values of $\sigma_{m} / \sigma_{0}$ 
The influence of the factor $n$ (see section 5.7 ), the Gauss integration order, and the number of plastic multipliers associated with each element, are summarized in Table 6.2.7. Sixteen analyses of the V-notched specimen were performed using the element configuration of Figure 6.2.9, to a load of $\sigma_{\mathrm{m}} / \sigma_{\mathrm{o}}=1,473$. Conditions of plane strain and no strain hardening were assumed. The columns of Table 6.2.I represent the following: analysis number; $n$, ratio of von Mises equivalent stress to yield stress for an elastic integration point to be treated as plastic; integration order; number of load increments to a final load of $\sigma_{\mathrm{m}} / \sigma_{0}=1,473$; number of plastic integration points at final load; number of plastic integration points at final load as percentage of total number of integration points; $u$, longitudinal displacement (in $\mathrm{mm}$ ) at end of specimen on axis of symmetry; and CPU time (mins:secs) to produce result on a UNIVAC 1106 computer.

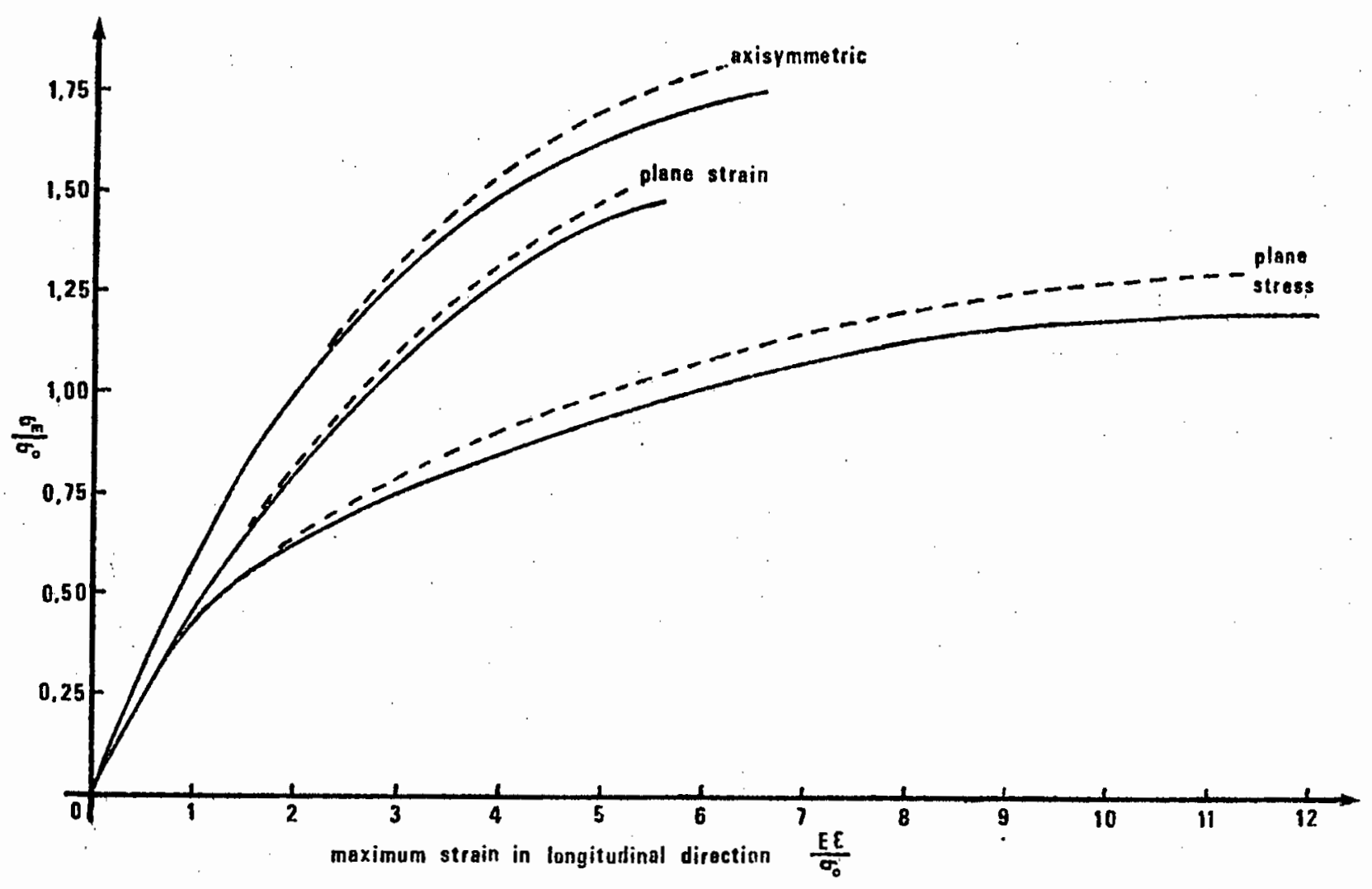

Figure 6.2.13 Load-strain curves for V-notched specimen, isoparametric finite element analysis 


\begin{tabular}{|c|c|c|c|c|c|c|c|c|}
\hline Analysis & $n$ & $\begin{array}{l}\text { Int. } \\
\text { order }\end{array}$ & $\lambda /$ elem. & $\begin{array}{l}\text { Load } \\
\text { incrmts. }\end{array}$ & $\begin{array}{l}\text { Plastic } \\
\text { int. pts. }\end{array}$ & $\%$ Plastic & $\mathrm{u}(\mathrm{mm})$ & $\begin{array}{l}\text { CPU time } \\
(\mathrm{m}: \mathrm{s})\end{array}$ \\
\hline 1 & 1,000 & 4 & 16 & 58 & 57 & 24 & 0,0927 & $10: 30$ \\
\hline 2 & 1,000 & 3 & 9 & 32 & 31 & 23 & 0,0929 & $3: 06$ \\
\hline 3 & 0,995 & 4 & 16 & 24 & 59 & 25 & 0,0929 & $3: 23$ \\
\hline 4 & 0,995 & 3 & 9 & 19 & 32 & 24 & 0,0930 & $1: 38$ \\
\hline 5 & 0,990 & 4 & 16 & 19 & 63 & 26 & 0,0931 & $3: 00$ \\
\hline 6 & 0,990 & 3 & 9 & 15 & 34 & 25 & 0,0933 & $1: 20$ \\
\hline 7 & 0,975 & 4 & 16 & 14 & 70 & 29 & 0,0942 & $2: 11$ \\
\hline 8 & 0,975 & 3 & 9 & 11 & 37 & 27 & 0,0942 & $1: 04$ \\
\hline 9 & 1,000 & 4 & 1 & 26 & 25 & 10 & 0,0905 & $1: 45$ \\
\hline 10 & 1,000 & 3 & 1 & 17 & 16 & 12 & 0,0906 & $0: 57$ \\
\hline 11 & 0,995 & 4 & 1 & 21 & 33 & 14 & 0,0904 & $1: 32$ \\
\hline 12 & 0,995 & 3 & 1 & 12 & 20 & 15 & 0,0906 & $0: 43$ \\
\hline 13 & 0,990 & 4 & 1 & 17 & 40 & 17 & 0,0905 & $1: 16$ \\
\hline 14 & 0,990 & 3 & 1 & 10 & 22 & 16 & 0,0907 & $0: 38$ \\
\hline 15 & 0,975 & 4 & 1 & 13 & 55 & 23 & 0,0907 & $1: 02$ \\
\hline .16 & 0,975 & 3 & 1 & 9 & $28^{\circ}$ & .21 & 0,0908 & $0: 38$ \\
\hline
\end{tabular}

Table 6.2.1 V-notched specimen, plane strain: analyses to $\sigma_{\mathrm{m}} / \sigma_{0}=1,473$ 
The plastic integration points of Figure 6.2.11 correspond to analysis 6 in the Table. It may be assumed that analysis 1 produced the most accurate results since fourth order integration, sixteen plastic multipliers per element, and fifty-eight load increments were required to reach the final load, taking a total of $10 \mathrm{~min} 30$ secs CPU time. With a small sacrifice in accuracy computation time can be drastically reduced. Consider, for example, analysis 6. This consists of third order integration, nine plastic multipliers per element, and a value of 0,990 for $n$. This reduced the number of load increments to fifteen, resulting in an $87 \%$ reduction in computation time. The sacrifice in accuracy amounted to a $1 \%$ difference in the percentage of plastic integration points, and $0,65 \%$ difference in deflection $u$. In analyses 9 to 16 it is apparent that the finite element mesh is too coarse to consider only one 'average' plastic multiplier per element. Although in these cases the deflection $u$ is reasonably close to the value of analysis 1 , the extent of the plastic region (shown by the percentage of plastic integration points) is not reliable. In comparison, the deflection $u$ corresponding to a triangular constant strain element analysis to the same load was found to be $0,0938 \mathrm{~mm}$.

Comparisons of computational efficiencies for different elastoplastic analysis techniques are not always meaningful. By considering an analysis method alone (and not the associated computer program) it is not possible to assess even approximate numbers of arithmetic operations to be performed, and thus the only measure of relative efficiency is in comparison of computation times required for the same numerical example. However, not only are program running times machine dependent, but certain quantities are dependent on the method of analysis - for example, number of stages in the analysis to a particular load. Thus strict comparisons of execution times for the present method and published results are not 


\begin{tabular}{|c|c|c|c|c|c|c|c|c|}
\hline Reference & Method & Element & Nodes & Elements & Load $\frac{\sigma_{m}}{\sigma_{0}}$ & Stages & Computer & Time (min) \\
\hline Marcal \& King & \multirow{5}{*}{$\begin{array}{l}\text { Tangent } \\
\text { Modulus }\end{array}$} & \multirow{5}{*}{$\begin{array}{c}\text { Constant } \\
\text { Strain } \\
\text { Triangle }\end{array}$} & 150 & 250 & 1,232 & 8 & \multirow{3}{*}{$\begin{array}{r}\text { IBM } \\
7090\end{array}$} & 15 \\
\hline Yamada et al & & & 149 & 259 & 1.192 & & & \\
\hline & & & & & & & & \\
\hline Yamada et al & & & 144 & 245 & 1,224 & 51 & $\begin{array}{l}\text { HITAC } \\
.5020 \mathrm{E}\end{array}$ & 29 \\
\hline Present analysis & & & 143 & 244 & 1,188 & 34 & \multirow{3}{*}{$\begin{array}{l}\text { UNIVAC } \\
1106\end{array}$} & בו \\
\hline Present analysis & $1 \lambda /$ int. pt. & \multirow{2}{*}{$\begin{array}{l}\text { Isopara- } \\
\text { metric }\end{array}$} & \multirow{2}{*}{100} & \multirow{2}{*}{15} & \multirow{2}{*}{1,257} & 23 & & 3,5 \\
\hline Present analysis & $I \lambda /$ element & & & & & 15 & & 1 \\
\hline Anand \& Shaw & Tangent & $\begin{array}{c}\text { inear Stro } \\
\text { Triangle }\end{array}$ & \multirow{3}{*}{172} & \multirow[t]{3}{*}{75} & 1,236 & - & \multirow{2}{*}{$\begin{array}{c}\text { IBM } \\
370 / 158\end{array}$} & 46,5 \\
\hline Anand \& Shaw & Modulus & Constant & & & & - & & 21,5 \\
\hline Present analysis & & $\begin{array}{l}\text { Strain } \\
\text { Triangle. }\end{array}$ & & & 1,210 & 20 & $\begin{array}{l}\text { UNIVAC } \\
1106\end{array}$ & 2,5 \\
\hline
\end{tabular}

Table 6.2.2 V-notched tension specimen in plane stress, no strain hardening 
meaningful. Further, numerical results in the literature generally do not state whether 'computation time' is taken to be total machine time or central processing unit (CPU) time, often significantly different. In this thesis all computation times given for results of the present analyses are CPU times.

In view of the above, computation times listed in Table 6.2.2 for the vee-notched specimen in plane stress should be assessed as only indicative of the order of magnitude of times required by the various analysis procedures. With this in mind the present approach appears attractive in terms of relative computational efficiency. More meaningful comparisons with published results for which similar computers were used, and to the same load level, will be given for the following numerical example.

\subsection{Deep Cantilever in Plane Stress}

The elasto-plastic analysis of a cantilever of rectangular crosssection in plane stress and with length to depth ratio equal to unity has been reported in the literature. Zienkiewicz and Valliapan (1971) [32] used a constant elasticity matrix initial stress method, whereas de Donato and Franchi (1973) [22] and de Donato and Maier (1973) [20] employed quadratic programming techniques to determine a solution. Using a finite difference technique Neal (1968) [33] established a lower bound for the limit load by determining loads corresponding to safe and statically admissible stress fields.

The cantilever depth $d$ is equal to the length $L$ as shown in Figure 6.3.1. The built-in condition is represented by an $L$ by $2 d$ plane of the same material, also of unit thickness, and fully constrained at the three internal boundaries. Loading consists of a vertical shear force distributed parabolically over the free end $\mathrm{BF}$ as shown in the Figure. 


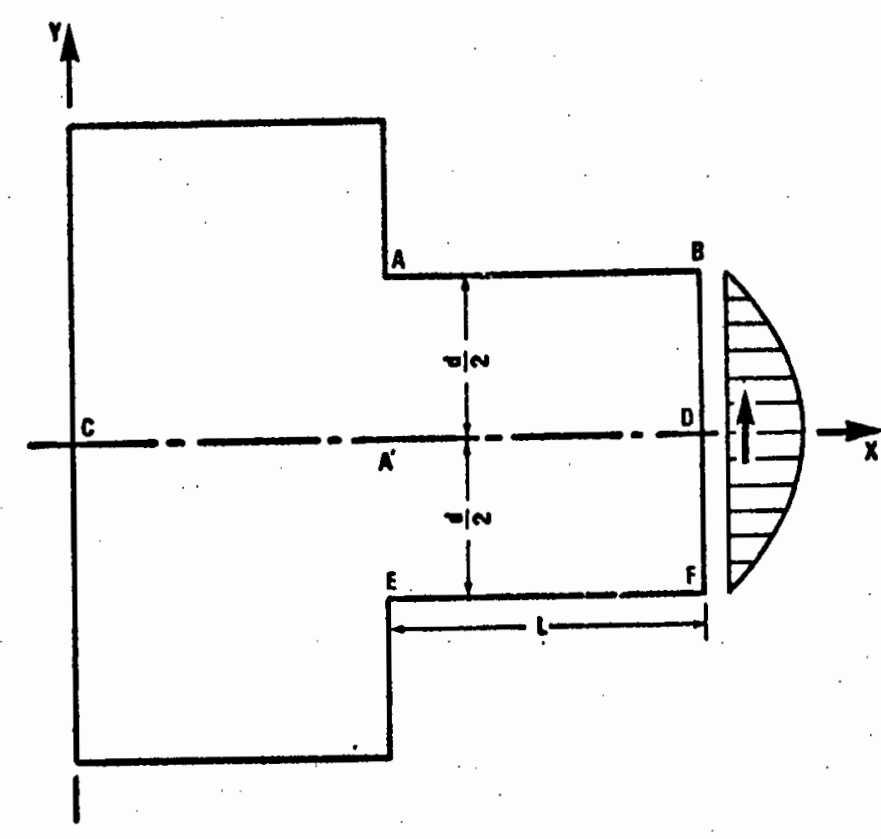

Figure 6.3.1 Deep cantilever in plane stress

If the magnitude of the applied shear stress at point $D$ is $\bar{s}$ then the total load is $w=\frac{2 d}{3} \bar{s}$, and the shear stress on $B F$ given by $\sigma_{x y}=\frac{\bar{s}}{d^{2}}\left(d^{2}-4 y^{2}\right)$. Constraining horizontal displacement components along the axis of symmetry $C D$, only one half of the structure need be considered.

In each of the papers cited above a mesh of 158 triangular constant strain finite elements and 97 nodes was used to represent the half structure. Mechanical properties for the elastic-perfectly plastic von Mises material were given as $E=1000 \mathrm{~kg} / \mathrm{mm}^{2} ; \nu=0,0$; and $\sigma_{0}=32 \mathrm{~kg} / \mathrm{mm}^{2}$. These values were used in the present analysis. Figures 6.3.2(a) and (b) respectively show configuration of the 158 constant strain elements, and an isoparametric element mesh consisting of 31 elements and 192 nodes. In both cases the element size is decreased in the region of point $A$, the extreme fibre' on the line of maximum moment $A A^{\prime}$. 'The parabolic end load is represented by equivalent nodal loads on $B D$. 


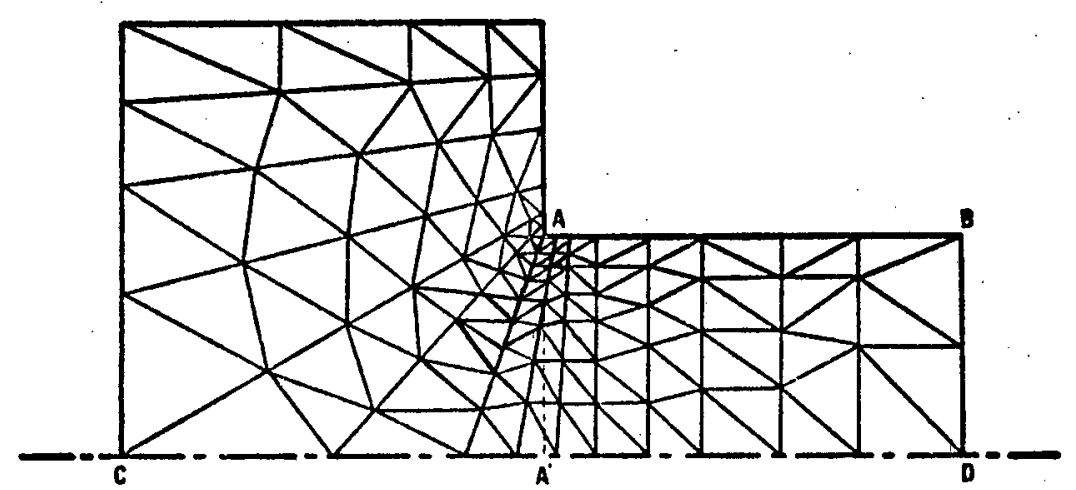

(a)

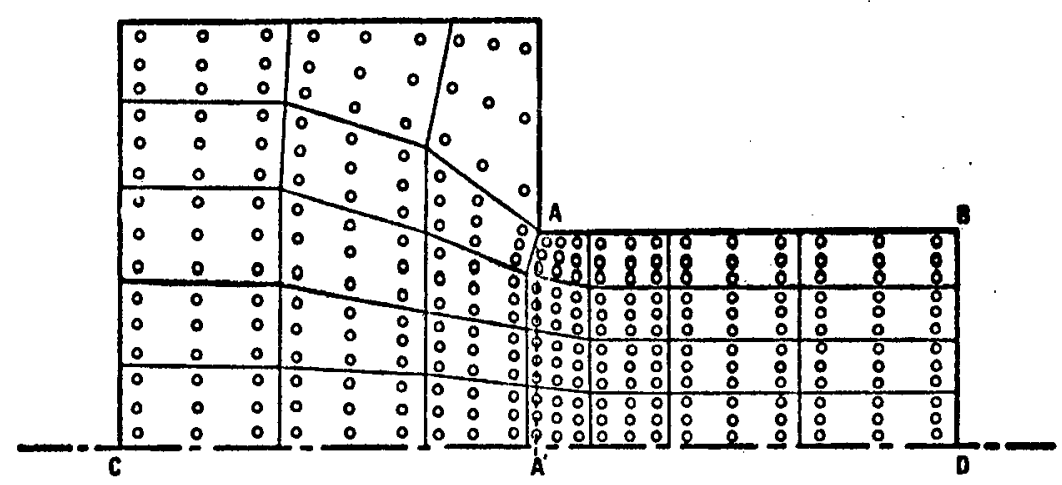

(b)

Figure 6.3.2 Finite element meshes

The lower bound on the limit load established by Neal is $M / M_{O}=0,921$; where $M=W L$ and $M_{0}$ is the fully plastic moment for the section given by $M_{0}=\frac{1}{4} b d^{2} \sigma_{0}$. Figure 6.3 .3 shows plastic regions of the cantilever resulting from various numerical analyses to this load level. Referring to this Figure, (a) results from the present analysis using constant strain elements. The effect of element orientation is again apparent. The plastic integration points of (b) and (c) are from isoparametric finite element analyses using the present method - in ( $b$ ) one plastic multiplier is associated with each integration point, while in (c) one average multiplier is associated with each element. The plastic enclave shown in (d) was obtained using the nonlinear computer 


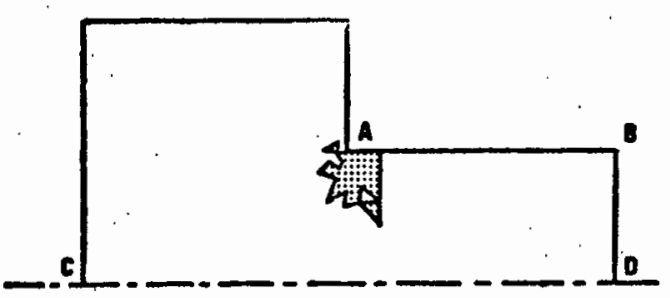

(a)

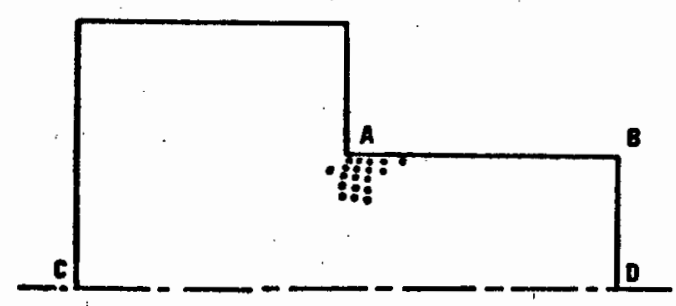

(c)

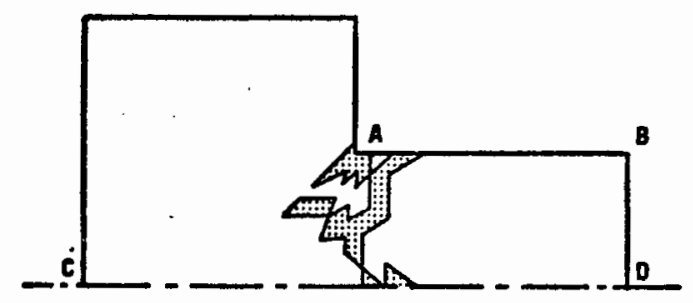

(e)

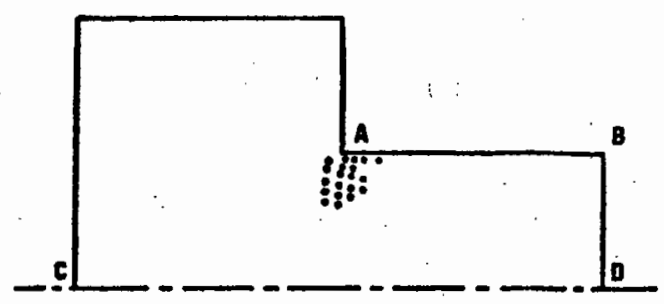

(b)

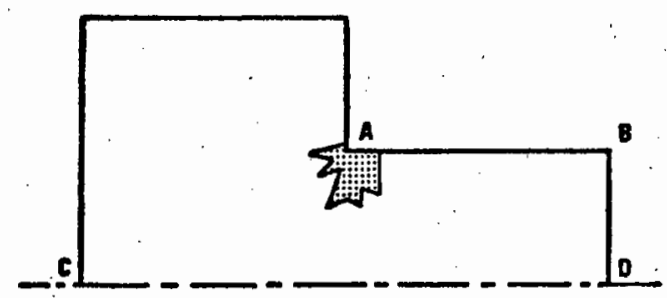

(d)

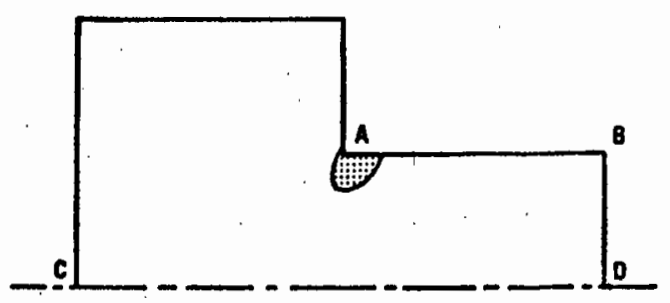

(f)

Figure 6.3.3 Plastic enclaves at $M / M_{0}=0,921$

(a) Present analysis, constant strain

(b) Present analysis, isoparametric, $1 \lambda$ /integration point

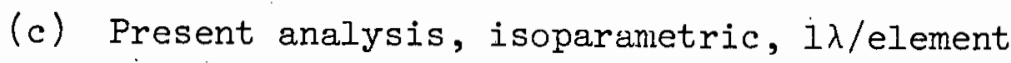

(d) Program INONSAP

(e) de Donato and Franchi

(f) Zienkiewicz and Valliapan, $M / M_{0}=0,929$ 
program NONSAP developed by Bathe [28], which uses a tangent modulus approach for material nonlinearity. A mesh of 158 triangular constant strain elements and 97 nodes was also used. The extent of the plastic region is in good agreement with those shown in (a), (b) and (c). At the same load level plastic elements from de Donato and Franchi are indicated in (e). This significantly larger and irregular plastic region is not in agreement with other results. The plastic enclave of ( $f$ ) was given by Zienkiewicz and Valliapan and corresponds to a slightly higher load $M / M_{0}=0,929$.

The horizontal stress $\sigma_{x}$ on the line AA' (see Figure 6.3.2) is plotted in dimensionless form in Figure 6.3 .4 for the load $M / \dot{M}_{0}=0,921$. Stresses from the present analyses are in good agreement with those resulting from the program NONSAP, but do not agree with those of the de Donato and Franchi or Neal, especially in the region which is not undergoing plastic deformation. However Neal's stress distribution results from limit analysis in which stresses in the elastic region are not known - any safe and statically admissible stress field is permissible for the lower bound solution.

Figure 6.3.5 shows displacement in the y-direction along the centre line $C D$ at the load $M / M_{0}=0,921$. Again results of the present analyses are in good agreement with Bathe's program NONSAP, but do not coincide with de Donato and Franchi.

To illustrate elastic unloading after plastic deformation the hysteresis loops $A$ and $B$ in Figure 6.3 .6 correspond to load cycles $A$ and $B$ shown diagrammatically in Figure 6.3.7. In both Figures the vertical axis is the applied load $M_{0} M_{0}$. The horizontal axis in Figure 6.3 .6 gives in dimensionless form vertical deflection at point $D$ on the free end of the cantilever (see Figure 6.3.1). These results are from a constant strain finite element analysis using 158 elements and 97 nodes. The total 


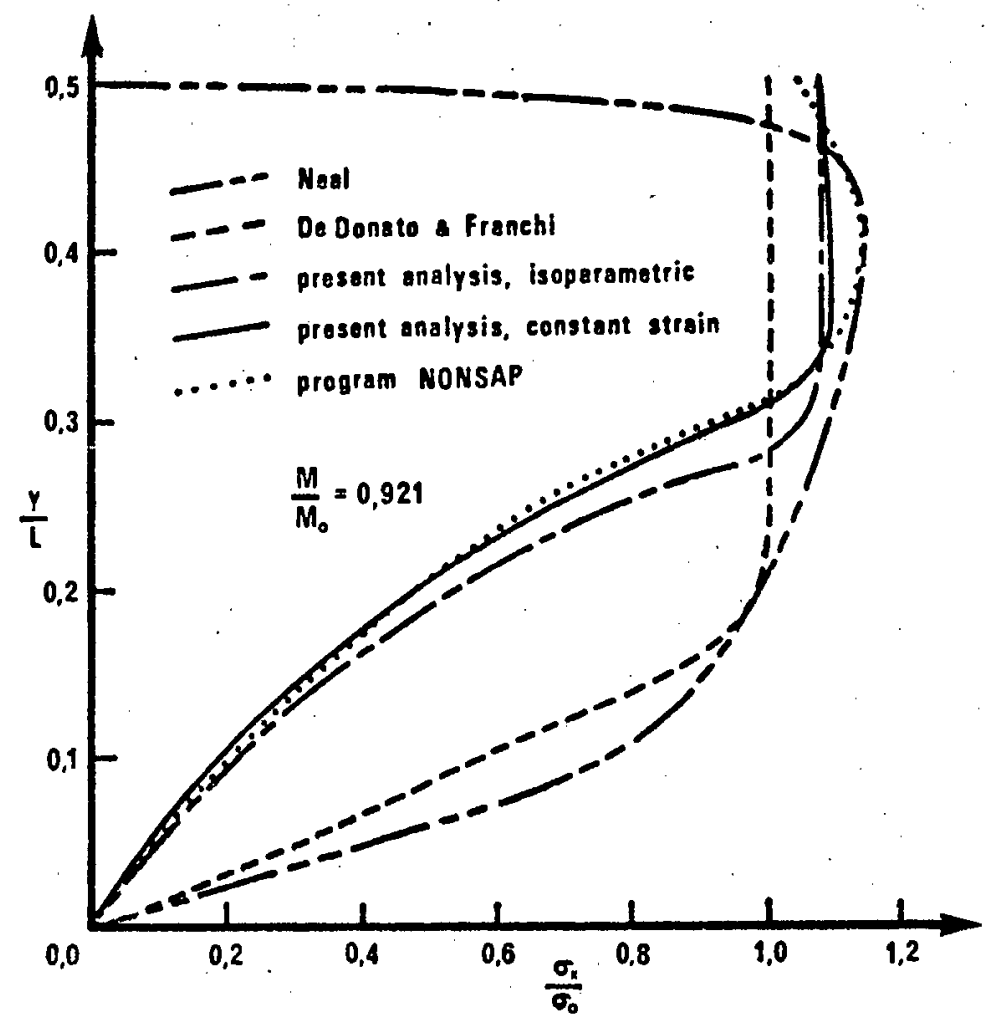

Figure 6.3.4 Horizontal stress on AA'

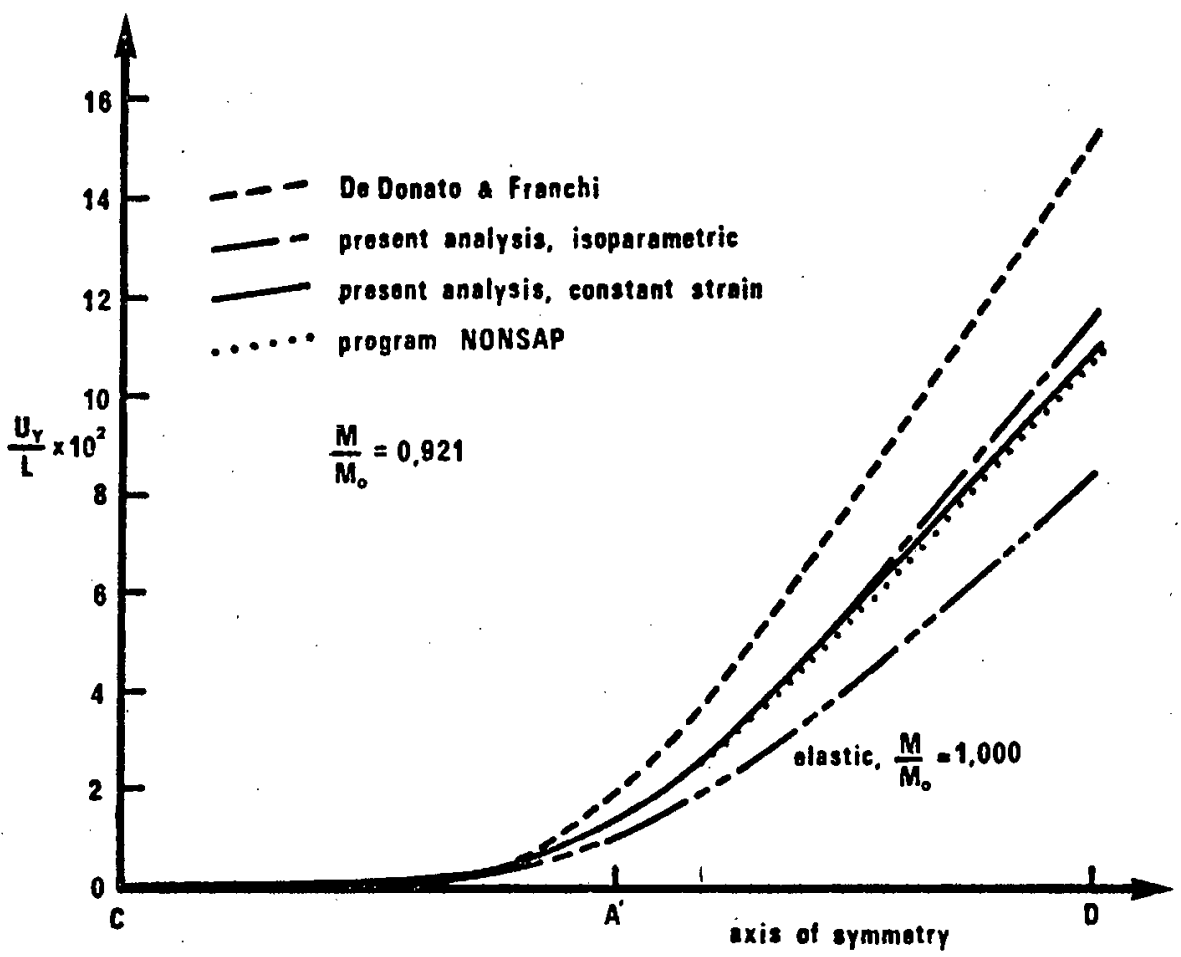

Figure 6.3.5 Displacement in y-direction along centre line CD; $M / M_{0}=0,921$ 


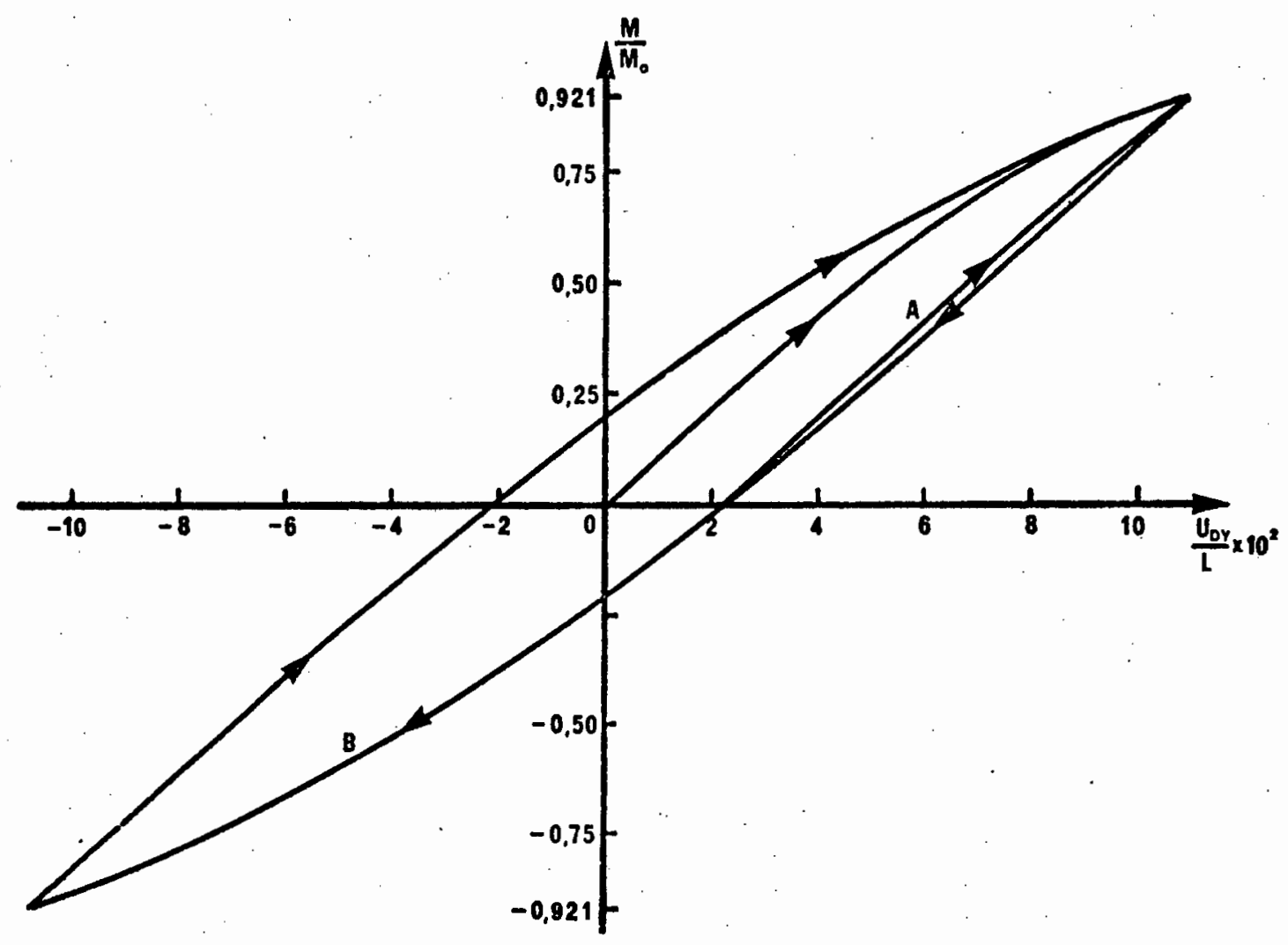

Figure 6.3.6 Load-deflection characteristics for cyclic loading

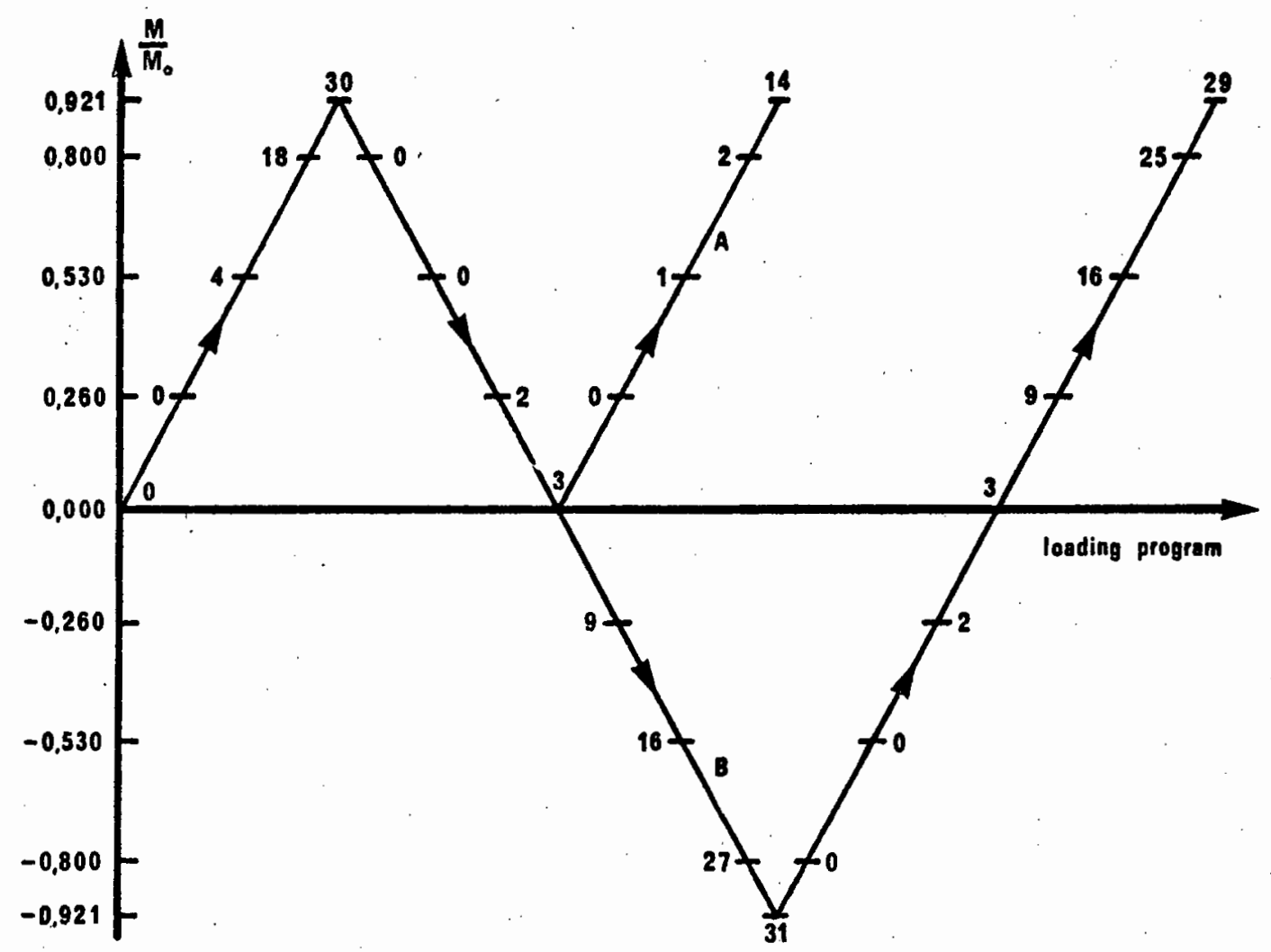

Figure 6.3.7 Cyclic loading programs $A$ and $B$ 
numbers of elements deforming plastically at representative values of load are given in the diagram of Figure 6.3.7.

Load cycle A begins from the initial unstressed and undeformed state, incrementing the load until $M_{M_{0}}=0,921$. At this stage thirty elements are plastic. The direction of loading is then reversed which causes all plastic elements to unload elastically. However, before the total load has become zero three elements again deform plastically. After reaching zero, load is then re-applied until $M_{1} M_{0}=0,921$. At this stage only fourteen elements become plastic again, while the vertical deflection at $D$ is within $0,2 \%$ of its value at termination of the first load direction of cycle A.

For load cycle $B$ the direction of loading is also reversed at $M / M_{0}=0,921$, but is then maintained in this direction until $M_{0} M_{0}=-0,921$. Load is then re-applied in the initial direction up to the value $M_{0} M_{0}=0,92 \ldots$. Cycles between the two extreme load values show similar numbers of plastic elements at corresponding load levels of loading or unloading.

Figures 6.3 .8 and 6.3 .9 give results obtained for the cantilever from isoparametric finite element analyses either associating a plastic multiplier $\lambda$ with each integration point, or associating one average multiplier with each element. From the load-deflection curve it is seen that using a single average multiplier for each element leads to an apparent increase in stiffness. Since even small regions undergoing large plastic strains can cause large displacements, decreasing these peak strain values by averaging the plastic multiplier over the element would cause the assemblage to exhibit stiffer load-deflection characteristics. Horizontal stresses $\sigma_{x}$ on the line $A A^{\prime}$ are plotted for two load levels in Figure 6.3 .9 and indicate the disparity in stress distribution for the two analyses. Since the elastic part of any strain increment is evaluated as the difference between increments in plastic strain and 


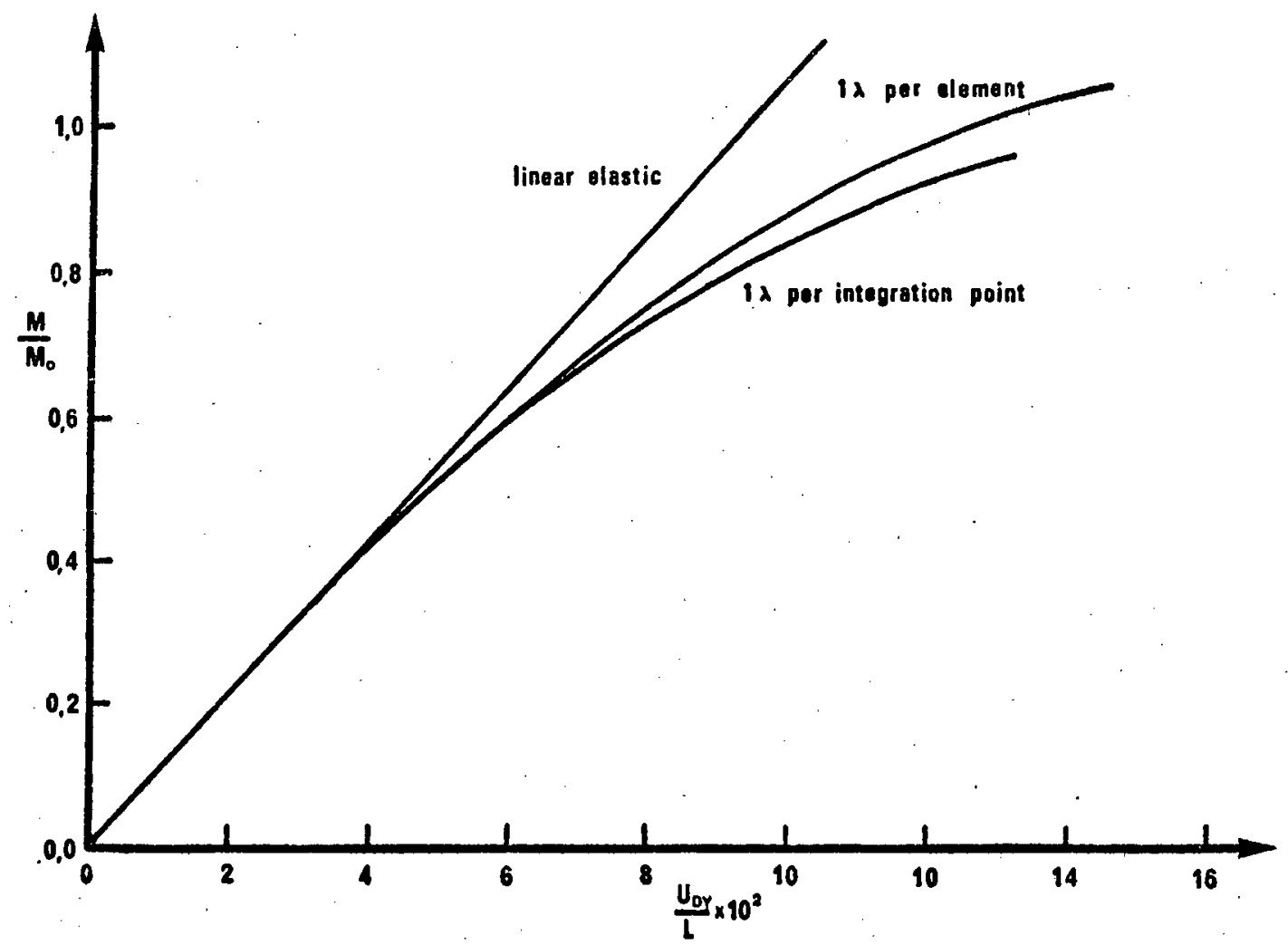

Figure 6.3.8 Load-deflection curves from isoparametric element analysis

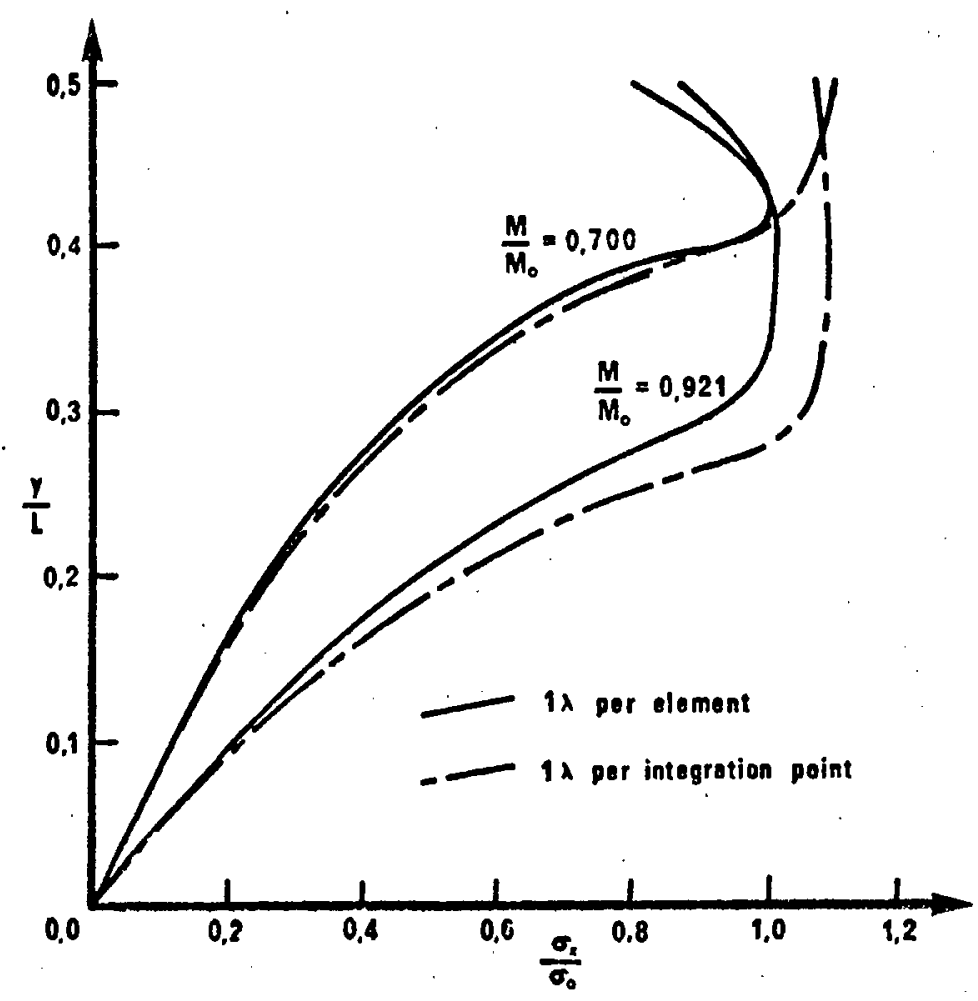

Figure 6.3.9 Horizontal stresses on AA' 
total strain, any error in plastic strain due to averaging plastic multipliers within an element introduces errors in stress increments and hence in total stresses. However this approach does provide reasonable estimates of such quantities as the extent of plastic regions and limit load for the elastic-perfectly plastic case, while requiring much less computer storage and computation time.

Plastic regions indicated in Figure 6.3.10 correspond to the stages at which the present analyses were terminated when successive load increment magnitudes become less than $0,5 \%$ of the total load applied. Load levels indicated are thus estimates of the limit load according to the analysis performed. In this Figure (a), (b) and (c) correspond respecitvely to the analysis of Figure $6.3 .3(\mathrm{a}),(\mathrm{b})$ and $(\mathrm{c})$. Since

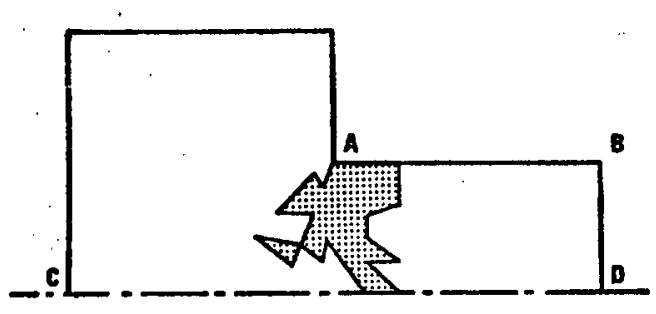

(a)

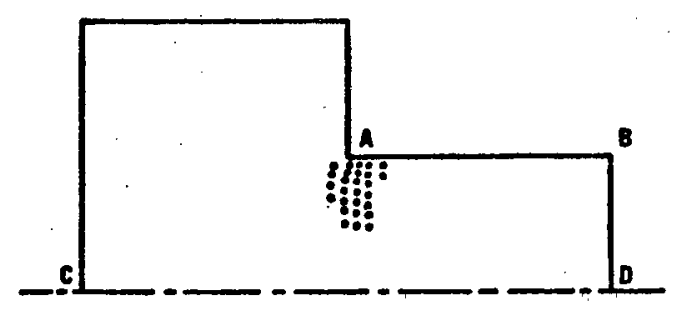

(b)

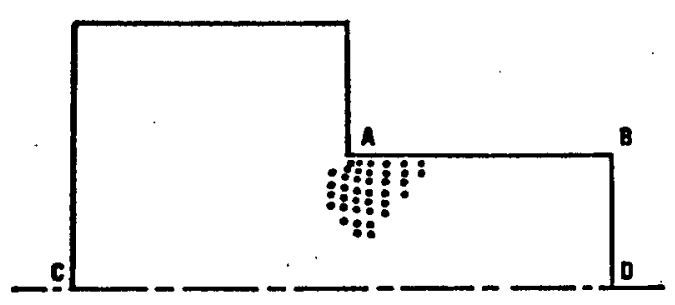

(c)

Figure 6.3.10 Plastic enclaves at termination of present analyses

(a) Constant strain elements, $M / M_{0}=1,083$

(b) Isoparametric elements, $I \lambda /$ integration point, $\mathrm{M}_{\mathrm{M}_{0}}=0,955$

(c). Isoparametric elements, $I \lambda /$ element, $M_{0} M_{O}=1,063$ 


\begin{tabular}{|c|c|c|c|c|c|c|c|c|}
\hline Reference & Method & Element & Nodes & Elements & Load $\frac{\mathrm{M}}{\mathrm{M}_{\mathrm{O}}}$ & Stages & Computer & $\begin{array}{l}\text { Time } \\
\text { (min:sec) }\end{array}$ \\
\hline $\begin{array}{l}\text { de Donato } \\
\text { \& Franchi }\end{array}$ & Q.P. & & 7 & 7 & & 6 & & $11: 43$ \\
\hline NONSAP ${ }^{+}$ & $\begin{array}{l}\text { Tangent } \\
\text { Modulus }\end{array}$ & $\begin{array}{c}\text { Constant } \\
\text { Strain } \\
\text { Triangle }\end{array}$ & 97 & 158 & & 26 & UNIVAC & $6: 48$ \\
\hline Present analysis & & & & ] & 0,921 & 26 & 1106 & $1: 37$ \\
\hline Present analysis & $1 \lambda /$ int.pt. & Isopara- & & 7 & & 18 & & $2: 28$ \\
\hline Present analysis & $1 \lambda /$ element & metr & 192 & 31 & & 17 & & $1: 38$ \\
\hline
\end{tabular}

† 8 node quadrilateral isoparametric degenerated to constant strain triangle

Table 6.3.1. Deep beam in plane stress 
the present formulation is derived from a kinematic minimum principle, these estimates of ultimate load correspond to upper bounds on the limit load, while Neal's lower bound value is $M / M_{0}=0,921$. The constant strain analysis required $5 \mathrm{~min} 58 \mathrm{sec}$ CPU time, and consisted of 43 load increments. The isoparametric analyses consisted of 24 load increments for the case of one plastic multiplier per integration point, taking $3 \mathrm{~min} 45 \mathrm{sec} C P U$ time; while using one average multiplier per element required $2 \min 45 \mathrm{sec}$ for a total of 29 load increments.

As noted previously categorical comparisons of computation times for the present analyses and published results cannot be made. However, the times listed in Table 6.3.1 are more meaningful than those given in Table 6.2.2 for the previous example, as in this case all analyses were performed to the same load $\left(\mathrm{M} / \mathrm{M}_{0}=0,921\right)$, and on similar computers. Constant strain triangular elements were not directly available in the program NONSAP, and therefore eight noded quadrilateral isoparametric elements were degenerated to form three noded constant strain triangles. In this way the same number of displacement degrees of freedom as the present constaint strain element analysis was ensured.

It is evident from the Table that in this case the present approach is significantly more efficient in terms of computation performed.

\subsection{Pressure Vessel - Flush Nozzle Junction}

An experimental investigation into the elasto-plastic behaviour of flush nozzles in spherical pressure vessels has been reported by Dinno and Gill [35]. A numerical analysis of one of these specimens has been performed by Nayak and Zienkiewicz [36], using eight noded parabolic quadrilateral isoparametric finite elements. The geometry of the specimen considered is shown in Figure 6.4.1, while the mechanical properties of the 


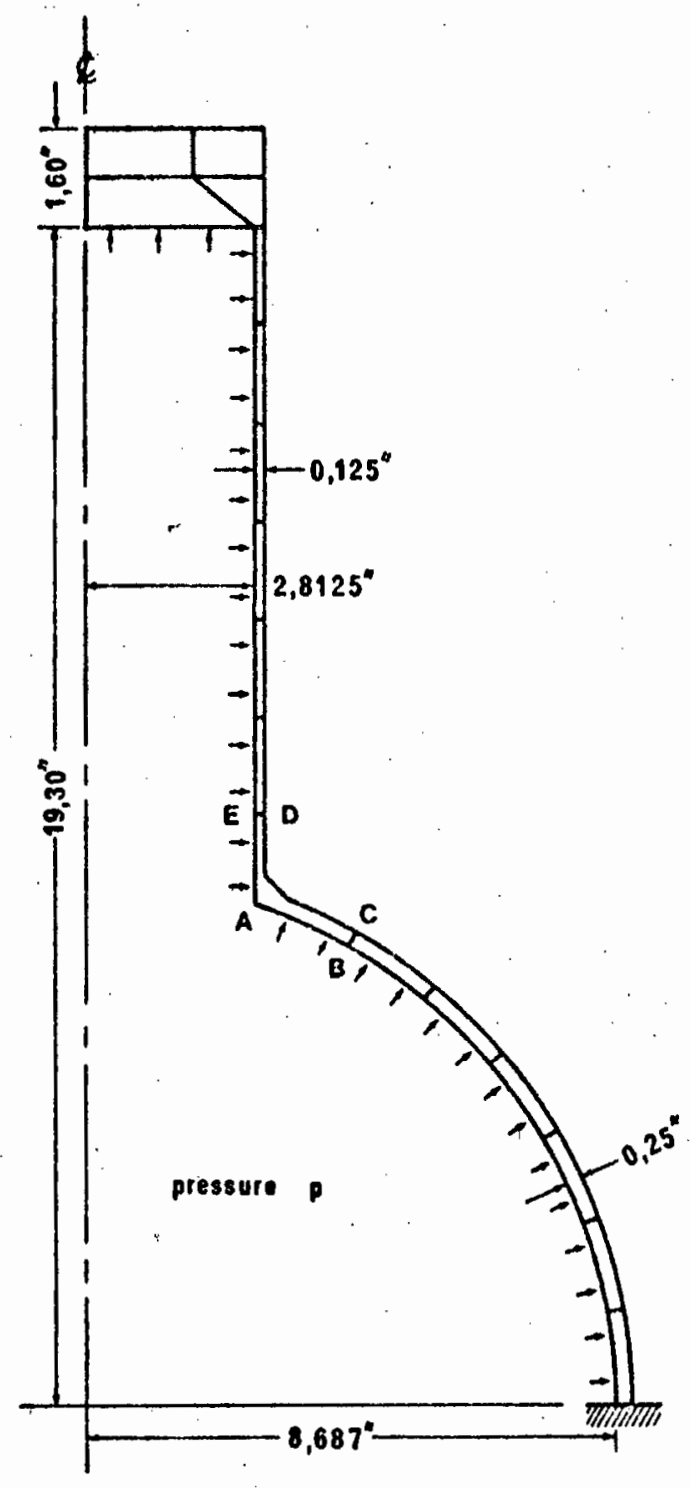

Figure 6.4.1 Spherical pressure vessel and cylindrical flush nozzle

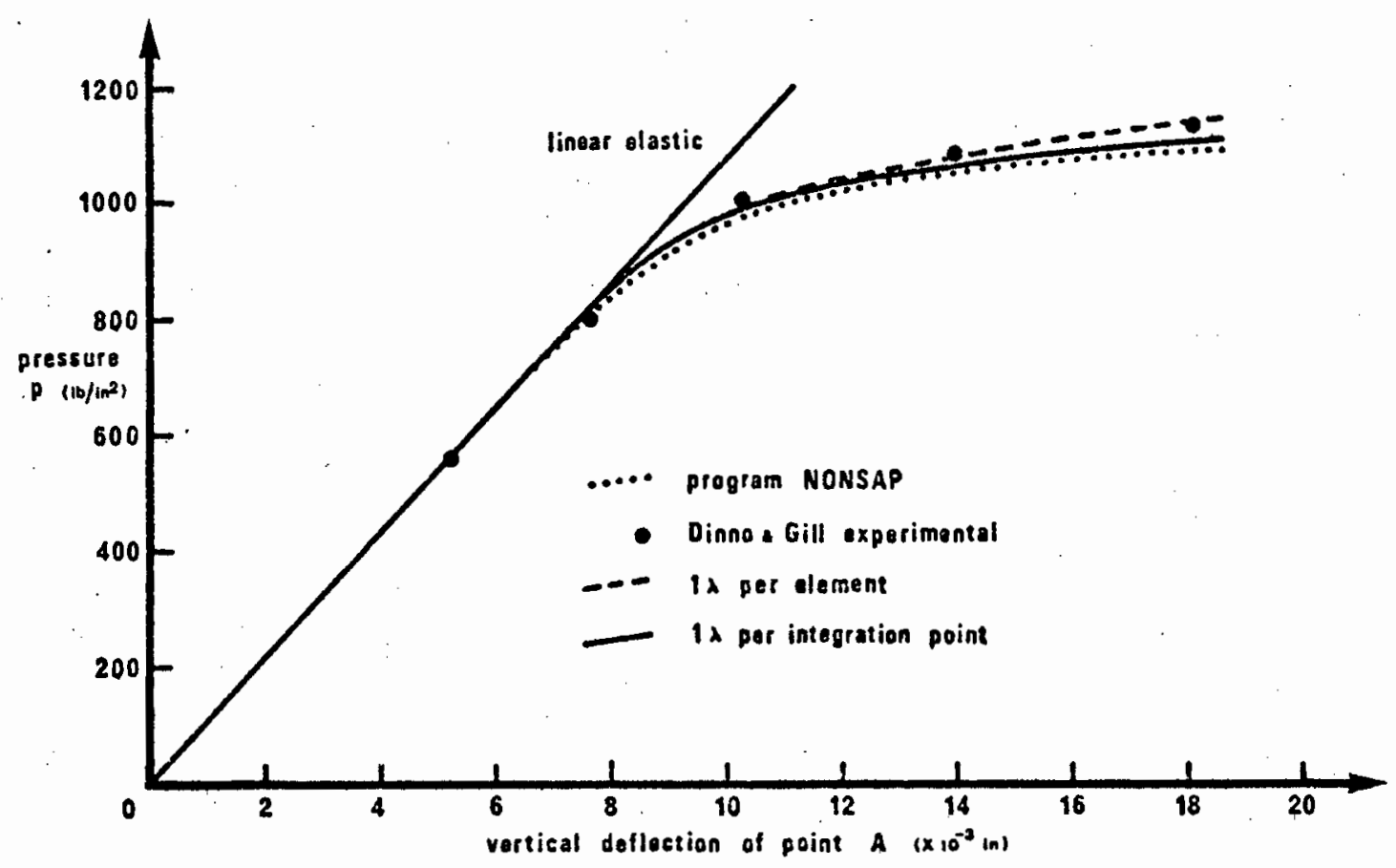

Figure 6.4.2 Pressure-displacement curve for pressure vessel - 
material were given as $E=29120000 \mathrm{lb} / \mathrm{in}^{2} ; \quad \nu=0,3$ and $\sigma_{0}=40540 \mathrm{lb} / \mathrm{in}$, with no strain hardening assumed. Loading of the axisymmetric specimen consisted of a uniform internal pressure.

Using the present method results were obtained for analyses of the vessel under increasing pressure using 26 cubic quadrilateral isoparametric elements and 209 nodes. This mesh is shown in Figure 6.4,1 with the enlargement of the weld region $A B C D E$ shown in Figure 6.4.3(c). So as to compare results an anlysis was performed using the program NONSAP, [28]. Since this program used eight noded parabolic quadrilateral elements a mesh was used similar to that used by Nayak and Zienkiewicz, consisting of 53 elements and 225 nodes. The element discretization of the weld region is shown in Figures $6.4 .3(a)$ and $(b)$.

In Figure 6.4.2 vertical displacement of point $A$ on the inside of the vessel is plotted against increasing internal pressure for various analyses. Excellent agreement between numerical and experimental results is obtained. Again use of one 'average' plastic multiplier for each element in the present method produces a stiffer analysis.

Under the loading considered plastic deformation is confined to the sphere-cylinder junction. The progression of plastic regions at increasing values of internal pressure is shown in the enlargements of the weld region in Figure 6.4.3. In this Figure (a) shows results of the analysis of Nayak and Zienkiewicz to a pressure of $1080 \mathrm{lb} / \mathrm{in}^{2}$, while (b) was obtained using the program NONSAP. Plastic enclaves from the present method are shown in (c) in which the elastic-plastic interfaces corresponding to analyses using one plastic multiplier per integration point or one 'average' multiplier per element are only slightly different at high pressures. (The dashed lines at pressures 1000 and $1080 \mathrm{ln} / \mathrm{in}^{2}$ correspond to the analysis using one multiplier per element). Possibly because this is essentially a thin shell problem good results are obtained using a coarse mesh of only 


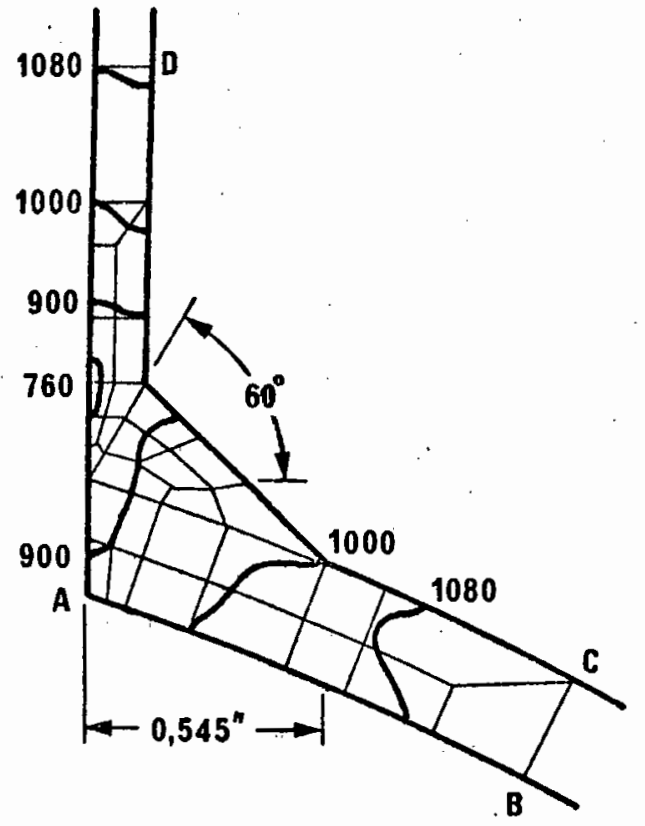

(a)

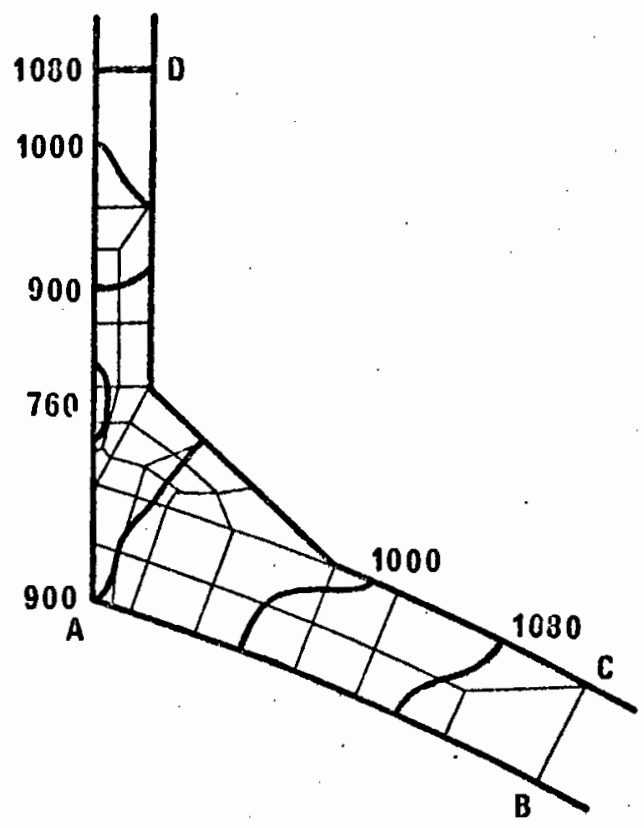

(b)

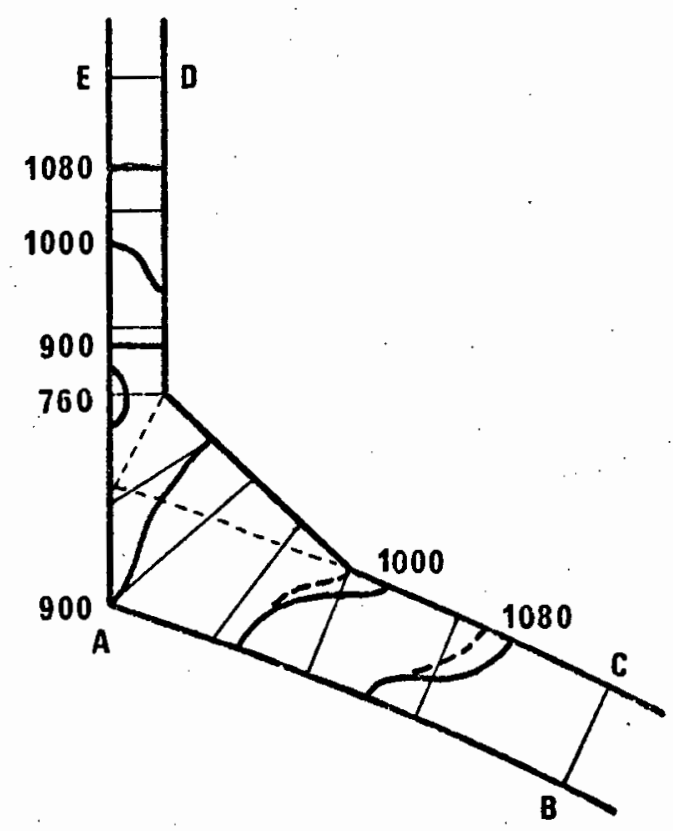

(c)

Figure 6.4.3 Plastic regions at values of internal pressure $\left(\mathrm{Ib} / \mathrm{in}^{2}\right)$

(a) Nayak and Zienkiewicz

(b) Program NONSAP

(c) Present analysis 
26 cubic quadrilateral elements. In particular use of one 'average' multiplier per element introduces negligible errors in this example local stress concentrations as well as over-all behaviour are adequately described.

The analysis using NONSAP consisted of 30 load increments to a pressure of $1080 \mathrm{lb} / \mathrm{in}^{2}$, and required $10 \mathrm{~min} 42 \mathrm{sec}$ CPU time on a UNIVAC 1106 computer. The present analyses to this value of internal pressure required 40 load increments and 11 min $22 \mathrm{sec} C P U$ time for the case of one multiplier per integration point; and 36 load increments and 3 min $24 \mathrm{sec}$ CPU time using one multiplier per element. These analyses were also performed using a UNIVAC 1106 computer. Nayak and Zienkiewicz did not give computation time required for their analysis. 
C H A P T E R 7

\section{DISCUSSION AND CONCLUSIONS}

An efficient method of solution has been presented for the elasto-plastic analysis of continua in plane stress, plane strain or axial symmetry. Application of the extended minimum principle to threedimensional continuum problems can be readily achieved by inclusion of field variable components ignored in the two-dimensional problem. The method appears attractive for three-dimensional analysis in view of the small increase in size of the system matrix in passing from the elastic to the elasto-plastic problem. In the formal quadratic programming approach the quadratic constraint on the value of the yield function is piecewise linearized, introducing a plastic multiplier and linear constraint for each yield plane of the assemblage, and thereby increasing the size of the problem manyfold.

Incremental plasticity requires determination of a complete solution for each load increment. In the conventional tangent modulus approach the entire system matrix is reformulated at each stage, while the initial strain/initial stress methods require iteration at every load increment. For the mathematical programming approach a linear complementary problem or quadratic programing problem must be solved at each stage. In the present method only part of the system matrix is reformulated for each increment. Further, the form of the system matrix is exploited to minimize computational effort, especially for load increments in which plastic deformations occur only in a small region of the assemblage. 
The algorithm used to determine a solution for the present programming technique seldom requires iteration for monotonically increasing loading. In this case elastic unloading occurs infrequently and thus the best initial assumption is that all plastic regions remain plastic for the next load increment. For numerical load cycling problems investigated, reversal of the load (which causes elastic unloading of the whole structure) was found to require a single iteration before the constraints were satisfied. In general the present approach thus reduces ta solving a set of simultaneous linear equations at each stage with possibly an occasional additional solution iteration. Although convergence of the algorithm has not been conclusively proved, it has been shown to be equivalent to the tangent modulus approach, which has wide acceptance. Failure to converge occurred only as the limit load was approached in the case of perfectly-plastic plane stress problems.

Exact comparisons of computational effort required by the present and other methods of analysis were not possible. But, from gross comparisons of computer times made for the numerical examples of the preceding chapter the order of magnitude of relative efficiency of the present method is clearly evident, while the ordex of accuracy is similar. From consideration of the form of the system matrix it is noted that problems for which the present method is especially suited are those in which plastic deformation is confined to small regions. This class of problem covers those in which stress concentrations occur, for example through abrupt change in geometry or through intersection of sections. In these problems of localized plastic deformation advantage is taken of the proposed solution method in which the system matrix is partitioned and the elastic part inverted once only. 
In the tangent modulus approach, if one has a priori knowledge of the region in which plastic deformation takes place, and further if it is possible to label finite element node numbers in such a way that this region is represented near the bottom of the banded system matrix, then a saving in computation can be achieved as the system matrix can be partially inverted once only. For each load increment change in the matrix to account for plastic elements is confined to the bottom of the band. The inversion is completed, and displacement increments evaluated by back substitution. However, labelling the nodes of a structure in this way without adversely affecting the band width of the elastic system matrix is usually not possible. The feasibility of this sheme is therefore entirely problem dependent.

Anand and Shaw [30] compared the use of constant strain and linear strain triangular finite elements in elasto-plastic solutions, using a tangent modulus approach. Contrary to their conclusion the results of the present method indicate that use of higher order elements is generally more efficient in terms of accuracy and computational expenditure. The use of one 'average' plastic multiplier associated with all plastic strains within an isoparametric element gives reasonable results while offering vast savings in computation and storage requirements. Further areas of research include the possibility of improving the accuracy of this latter technique by redistributing the 'average' multiplier according to some scheme, thereby improving the approximate variation in plastic strains across an element. 
REFERENCES

1. PRAGER, W., 'Fundamental theorems of a new mathematical theory of plasticity, Duke Math. Jnl., 9, 1942, 228.

2. PRAGER, W., 'Variational principles in the theory of plasticity', Proc. 6th Int. Congr. Appl. Mech., Paris, 1946.

3. HODGE, P.G. and PRAGER, W., 'A variational principle for plastic materials with strain hardening', J. Math. \& Phys., 27, 1948, 1.

4. GREEIRERG, H.J., 'On the variational principles of plasticity', Tech. Report All-54,' Div. of Appl. Math., Brown Univ., 1949.

5. GREENBERG, H.J., 'Complementary minimum principles for an elastoplastic material', Quart. Appl. Math., I, 1949, 85.

6. KOITER, W.T., 'Stress-strain relations, uniqueness and variational theorems for elasto-plastic materials with a singular yield surface', Quart. Appl. Math., 11, 1953, 350.

7. CERADINI, G., 'A maximum principle for the analysis of elasticplastic systems', Meccanica, $1,1966,77$.

8. MAIER, G., 'Some theorems for plastic strain rates and plastic strains', J. de Mecanique, $8,1969.5$.

9. GALLAGHER, R.H., PADLOG, J. and BIJLAARD, P.P., 'Stress analysis of heated complex shapes', Jnl. Amer. Rocket Soc., 32, 1962, 700.

10. ANGYRIS, J.H., 'Elasto-plastic displacement analysis of three dimensional continua', Jnl. Royal Aeronaut. Soc., 69, 1965, 633.

11. ZIENKIEWICZ, O.C., VALLIAPAN, S. and KING, I.P., 'Elasto plastic solutions of engineering problems initial stress, finite element approach', Int. J. Num. Meth. Eng., 1, 1969, 75.

12. POPE, G.G., 'A discrete element method for the analysis of plane elasto-plastic stress problems', The Aeronautics Quart., 11, 1, $1966 ; 83$. 
13. SWEDLOW, J.L., 'Elastic-plastic cracked plates in plane strain', Int. Jnl. Fracture Mech., 2, 1, 1969, 33.

14. MARCAL, P.V. and KING, I.P., 'Elastic-plastic analysis of twodimensional stress systems by the finite element method', Int. J. Mech. Sci, 2, 3, 1967, 143.

15. YAMADA, Y. YOSHIMURA, N. and SAKURAI, T., 'Plastic stress-strain matrix and its application for the solution of elastic-plastic probiems by the finite element method', Int. J. Mech. Sci., 10, 1968, 343,

16. MARCAL, P.V., 'A comparative study of numerical method of elasticplastic analysis', AIAA Jnl., $\underline{6}, 1968,157$.

17. HODGE, P.G., BELYTSCHKO, T. and HERAKOVICH, C., 'Plasticity and quadratic programming', Proc. Computational Approaches in Appl. Mech., (ASME), 73, 1969 .

18. SAYEGH, A.F. and RUBENSTEIN, M., 'Elastic-plastic analysis by quadratic programming', Proc. Eng. Mech. Div. ASCE., 98, (EM6), $1972,1547$.

19. GIACOMINI, S., MAIER, G. and PATERLINI, F., 'Complete solutions of elastic-plastic discretized structures by linear programming', presented at SMIRT 4, San Francisco, 1977.

20. DE DONATO, O. and MAIER, G., 'Finite element elastoplastic analysis by quadratic programming: the multistage method', presented at 2nd Int. Conf. Struct. Mech. in Reactor Technology, Berlin, 1973.

21. ANAND, S.C. and GARG, V.K., 'Shakedown of rolling wheel under hub loading', Jnl. Eng. Mech. Div., 100, (EM6), 1974, 1237.

22. DE DONATO, $O$. and FRANCHI, A., 'A modified gradient method for finite element elastoplastic analysis by quadratic programming', Comp. Meth. Appl. Mech. \& Eng., 2, 1973, 107.

23. MARTIN, J.B., 'On the kinematic minimum principle for the rate problem in classical plasticity', J. Mech. Phys. Solids, 23, 1975, 123.

24. MARTIN, J.B. and REDDY, B.D., 'A programming approach to the solution of the rate problem in elastic plastic solids', Mechanics in Engineering, ed. R.N. Dubey and N.C. Lind, Univ. of Waterloo Press, Canada, 1977.

25. MARTIN, J.B., 'Plasticity', MIT Press, 1975. 
26. VON MISES, R., 'Mechanik der festen Körper in plastisch deformablen Zustand', Göttinger Nachr. Math. Phys. Kl., 1913, 582.

27. TIMOSHENKO, S. and GOODIER, J.N., 'Theory of Elasticity', McGraw Hill, 2nd edition, 1951.

28. BATHE, K.J., WILSON, E.L. and IDLING, R.H., 'NONSAP a strucutral analysis program for static and dynamic response of nonlinear systems', Report no. UC SESM 74-3, Struc. Eng. Lab., Univ. Cal., Berkeley, 1974.

29. BATHE, K.J. and WILSON, L., 'Numerical Methods in Finite Element Analysis', Prentice Hall, 1976.

30. ANAND, S.C. and SHAW, R.H., 'Use of linear strain triangular elements in elasto-plastic solutions', to be published in Comp. Meth. Appl. Mech. and Eng.

31. THEOKARIS, P.A. and MARKETOS, E., 'Elastic-plastic strain and stress distribution in notched plates under plane stress', J.Mech. Phys. Solids, 11, 1963, 411.

32. ZIENKIEWICZ, O.C. and VALLIAPAN, S., 'Analysis of real structures for creep, plasticity and other complex constitutive laws', Strucutre, Solid Mechanics and Engineering Design, ed. M. Te'eni, Wiley Interscience, 1971, 27.

33. NEAL, B.G., 'Limit load of a cantilever in plane stress', in Engineering Plasticity, Cambridge Univ. Press, 1968, 473.

34. ZIENKIEWICZ, O.C., 'The Finite Element Method in Engineering Science', McGraw Hil1, 1971.

35. DINNO, K.S. and GILL, S.S., 'An experimental investigation into the plastic behaviour of flush nozzles in spherical pressure vessels', Int. J. Mech. Sci., I, 1965, 817.

36. NAYAK, G.C. and ZIENKIEWICZ, O.C., 'Elasto-plastic stress analysis. A generalization for various constitutive relations including strain softening', Int. Jnl. Num. Meth. Eng., 2, 1972, 113.

37. DITTMER, C.T. and MARTIN, J.B., 'A programming approach to the analysis of elasto-plastic continua by means of an extended kinematic minimum principle', presented at NATO-ASI on Plasticity and Math. Programming, Univ. Waterloo, Canada, 1977. 


\section{A.1. General Description}

The computer programs EPTCS (Elasto-Plastic analysis using Triangular Constant Strain finite elements) and EPCQI (Elasto-Plastic analysis using Cubic Quadrilateral Isoparametric finite elements) may be used for the incremental elasto-plastic analysis of continua in plane stress plane strain or axial symmetry. The material of the body must be either elastic-perfectly plastic or linear kinematic hardening, and is assumed to obey the von Mises yield condition. Loading of the assemblage consists of a series of piecewise linear proportional load paths. The magnitudes of individual load increments are determined internally during execution of the program.

The programs are written in FORTRAN $\mathrm{V}$ as implemented on the UNIVAC 1106 computer. This computer has a thirty-six bit word which gives eight significant figures for arithmetic performed in single precision. Moreover, the set of simultaneous linear equations to be solved for each load increment is well conditioned (unless the limit load is approached in the elastic-perfectly plastic case). Thus, excluding special algebraic operations such as the square root function, all arithmetic in the programs EPTCS and EPCQI is performed in single precision without loss of accuracy. The overall logic of each of the programs EPTCS and EPCQI is similar, but computational details differ. Each program consists of independent external subroutines linked by a main program. The function of each of the subroutines and the sequence in which they are called from the main program, is described in broad outline by means of the macro-flowchart in Figure A.1. Complete listings of EPTCS and EPCQI are given in sections (A.5) and (A.6) respectively. 


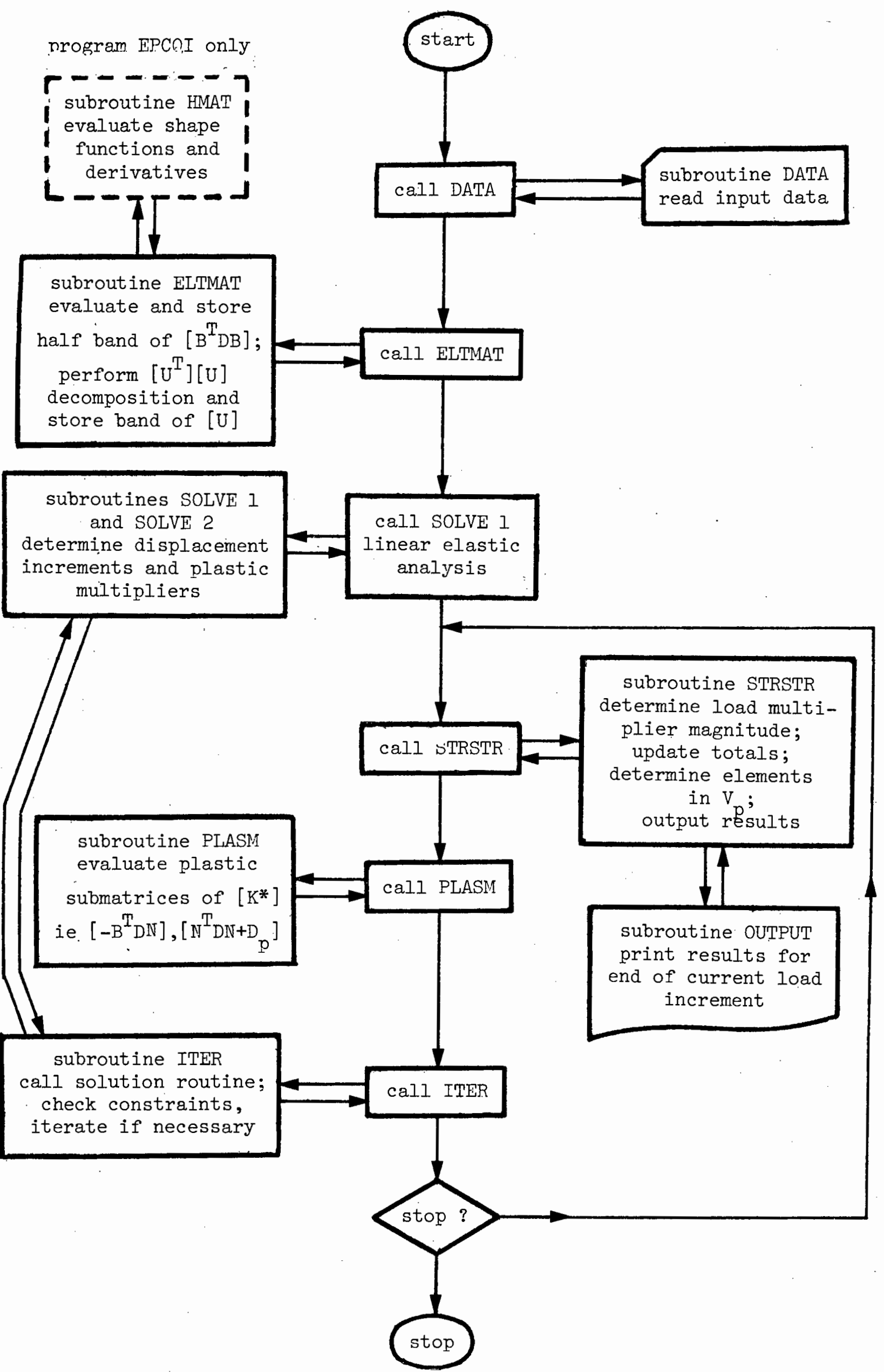




\section{A.2. Storage Allocation, Dimensioning of Arrays, and Execution}

For any realistic problem the programs require relatively large amounts of core storage. Therefore it is expedient to adjust dimensions of arrays to suit each particular analysis problem, thereby minimizing storage requirements.

To implement this dynamic dimensioning of arrays is employed where possible. Values of dynamic parameters are defined in the MAIN program, lines 5 to 11 for program EPTCS, or Iines 5 to 12 for program EPCQI; (see program listings sections A.5 and A.6). The parameters have the following representation:

NDFP maximum number of displacement degrees of freedom (i.e. maximum number of nodes multiplied by two)

NECP maximum half-band width of elastic system stiffness matrix $\left[B^{\mathrm{T}} \mathrm{DB}\right]$

NPCP maximum number of plastic multipliers to be determined for any load increment

NNP maximum number of nodes

NEP maximum number of elements

ISP maximum number of stress components considered at a point in the body, i.e. ISP $=3$ for plane stress, ISP $=4$ for plane strain or axial symmetry

NLPP maximum number of piecewise linear proportional load paths, plus one

IOP (for program EPCQI only) maximum integration order for Gauss quadrature; maximum value 4 , recommended value 3

For each analysis problem the values of these parameters should be adjusted to ensure sufficient storage allocation. During program execution dimension limits of arrays and values of parameters are checked to 
ensure they are compatible with the data input. If not a relevant error message is printed and the analysis terminated.

On the UNIVAC 1106 the collector limits direct core storage access to $64 \mathrm{~K}$ words. However, with extended storage facilities approximately $130 \mathrm{~K}$ words can be accessed in core. For this reason the two largest submatrices $\left[\mathrm{B}^{\mathrm{T}} \mathrm{DB}\right]$ and $\left[-\mathrm{B}^{\mathrm{T}} \mathrm{DN}\right]$ are assigned to external core storage in arrays named RKE and RKP respectively. This is achieved by the inclusion of a FORTRAN $V$ statement COMPILER $(X M=3)$ at the head of each routine in which the arrays RKE and RKP appear. Further, these two arrays must be dimensioned explicitly (parameters are not permitted) in the first labelled common block EXT. Arrays RKE and RKP appear in subroutines MAIN, ELTMAT, SOLVEI, SOLVE2, STRSTR and ITER. When altering the dimension limits of the programs each of these elements must be edited and the explicit dimensions given for RKE and RKP, each a two-dimensional array. The dimension limits for RKE must be the same as the values of parameters (NDFP, NECP); while those for RKP must be the same as the values of parameters (NDFP, NPCP). (see program listings, sections A.5 and A.6).

After FORTRAN compilation of the altered symbolic elements, all relocatable elements are collected into an absolute element $A B S$, ensuring that the external labelled common block EXT is assigned at the top of the data bank. The programs are collected as follows: QMAP,IE EPTCS.ABS

IN EPTCS.MAIN

IN EPTCS.DATA

IN EPTCS.ELTMAT

IN EPTCS.SOLVEI

IN EPTCS.SOLVE2

IN EPTCS.STRSTR

IN EPTCS.PLASM

IN EPTCS.ITER

IN EPTCS.OUTPUT

IN EXT
QMAP, IE EPCQI.ABS

IN EPCQI.MAIN

IN EPCQI.DATA

IN EPCQI.ELTMAT

IN EPCQI. HMAT

IN EPCQI.SOLVEI

IN EPCQI.SOLVE2

IN EPCQI.STRSTR

IN EPCQI.PLASM

IN EPCQI.ITER

IN EPCQI.OUTPUT

IN EXT 
Peripheral drum storage files are used to store the half band of the elastic system matrix $\left[\mathrm{B}^{\mathrm{T}} \mathrm{DB}\right]$, the band of the triangular decomposed matrix [U], the plastic system matrix $\left[-\mathrm{B}^{\mathrm{T}} \mathrm{DN}\right]$, and for the isoparametric case the element deformation matrices $\left[\mathrm{B}^{\prime}\right]$. The logical-unit numbers associated with these files are $11,12,13$ for the program EPTCS and 11 , $12,13,14$ for the program EPCQI. (These logical-unit numbers are specified in the MAIN programs).

Thus typical runstreams for execution of the programs on a UNIVAC machine are as follows:
QRUN
@RUN
@ASG,A EPTCS.
@ASG, A EPQCI.
@ASG,T 11
QASG,T 11
@ASG,T 12
QASG,T 12
@ASG,T 13
@ASG,T 13
@XQT EPTCS.ABS
@ASG,T 14
@XQT EPQCI.ABS

data

@FIN

data

@FIN

Data input is in free format, separated by blanks or commas. The programs are independent of the units of the input data, and thus units of the output will be consistent with those of input data.

\section{Turn to page A-23 for EPCQI}

\section{A.3. Program EPTCS}

The program EPTCS does not have an automatic triangular finite element mesh-generation capability, and thus nodal coordinates as well as element incidences are given explicitly as data. Data input required for execution may be divided into the seven subgraps listed in the following 
section (A.3.1), and described in section (A.3.2). These sections should be read in conjunction with the example of data input for EPTCS listed in section (A.3.3).

\section{A.3.1 Data Input for EPTCS}

\section{HEADINGI}

HEADING2

TYPE

NN , NE , E , EP , RNU, SZERO, ETA, (THIK) , FRAC

$\mathrm{X}_{1}, \mathrm{Y}_{1}, \mathrm{X}_{2}, \mathrm{Y}_{2} \cdot \cdot \cdot \cdot \cdot$

-

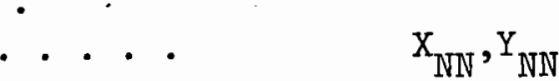

$\mathrm{IA}_{1,1}, \mathrm{IA}_{1,2}, \mathrm{IA}_{1,3} \mathrm{IA}_{2,1}, \mathrm{IA}_{2,2}$

$\bullet$

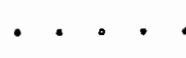

$\mathrm{IA}_{\mathrm{NE}, 1}, \mathrm{IA}_{\mathrm{NE}, 2}, \mathrm{IA}_{\mathrm{NE}, 3}$ i) heading statements

ii) structure statements

IX, IY

$\left.N_{a}, N_{b}, N_{c}, \ldots, N_{k},-1\right]$

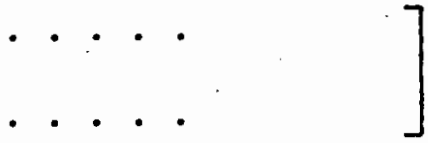

v) boundary conditions

$-1$

$\int$ iii) nodal coordinates

iv) element incidences

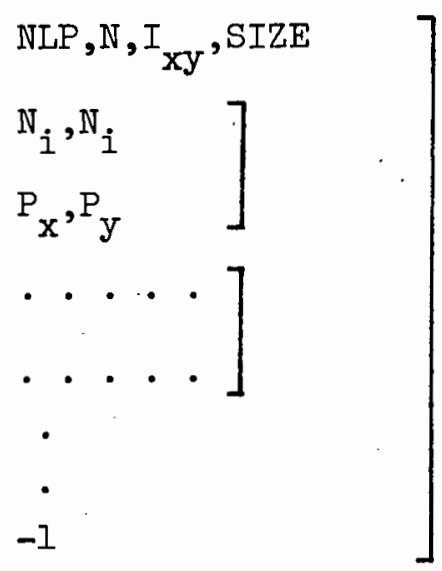




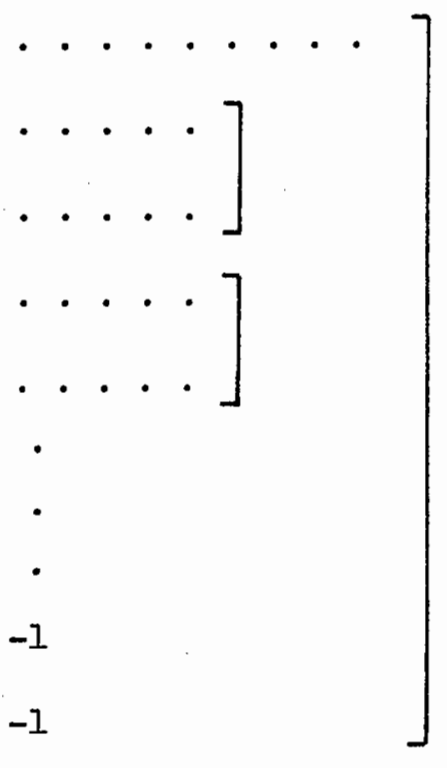

vi) loading program

PRINT

$$
\begin{aligned}
& I E_{a}, I E_{b}, I E_{c}, \ldots,{I E_{k},-1} \\
& N_{a}, \mathbb{N}_{b}, N_{c}, \ldots, N_{k},-1 \\
& \text { IOUT }_{a}, \text { IOUT }_{b}, \text { IOUT }_{c}, \ldots, \text { IOUT }_{k},-2
\end{aligned}
$$

vii) output requested

\section{A.3.2 Description of Data Input}

\section{(i) Heading Statements $\left.\begin{array}{l}\text { HEADING1 } \\ \text { HEADING2 }\end{array}\right]$}

where

HEADINGl each a string of alphanumeric characters of maximum length HEADING2 eighty. Can be used to identify problem, record units employed etc. Printed at top of output.

\section{(ii) Structure Statements}

TYPE

NN, NE , E , EP, RNU, SZERO, ETA , (THIK) , FRAC

where

TYPE (alpha) either PLANE STRESS, PLANE STRAIN OR AXISYMMETRIC

NN (integer) total number of nodes 


$\begin{array}{ll}N E \text { (integer) } & \text { total number of elements } \\ \text { E (real) } & \text { Young's modulus } \\ \text { EP (real) } & \text { plastic modulus for linear kinematic hardening; } \\ & \text { for elastic-perfectly plastic case enter } 0.0 \\ \text { RNU (real) } & \text { Poisson's ratio } \\ \text { SZERO (real) } & \text { uniaxial yield stress } \\ \text { ETA (real) } & \text { n, ratio of von Mises equivalent stress to yield stress } \\ & \text { for an elastic stress point to be treated as plastic. } \\ & \text { Recommended value } 0.99 \quad \text { (see section } 5.7 \text { ) } \\ & \text { plate thickness if plane stress analysis. Omit for } \\ & \text { plane strain or axisymetric analysis } \\ & \text { for determining the limit load in the elastic-perfectly } \\ & \text { plastic case. The analysis is terminated if for three } \\ & \text { successive load increments the ratio of load increment } \\ \text { FRAC (real) } & \text { magnitude to total load magnitude is less than the value of } \\ & \text { hardening. }\end{array}$

\section{(iii) Nodal Coordinates}

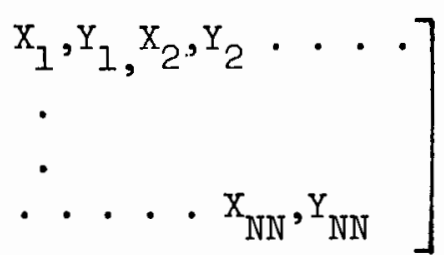

where

$X_{i}, Y_{i}$ (reals) are global Cartesian coordinates of ith node

\section{(iv) Element Incidences}

$$
\left.\begin{array}{l}
\mathrm{IA}_{1,1}, \mathrm{IA}_{1,2}, \mathrm{IA}_{1,3} \mathrm{IA}_{2,1}, \mathrm{IA}_{2,2} \ldots . \\
\therefore . \mathrm{IA}_{\mathrm{NE}, 1}, \mathrm{IA}_{\mathrm{NE}, 2}, \mathrm{IA}_{\mathrm{NE}, 3}
\end{array}\right]
$$

where 
$I A_{i, 1}, I A_{i, 2}, I A_{i, 3}$ (integers) are node numbers of apices of $i$ th element in counter-clockwise order.

(v) Boundary Conditions

$\mathrm{IX}, \mathrm{IY}$
$\left.\mathrm{N}_{\mathrm{a}}, \mathrm{N}_{\mathrm{b}}, \mathrm{N}_{c}, \ldots, \mathrm{N}_{k},-1\right]$

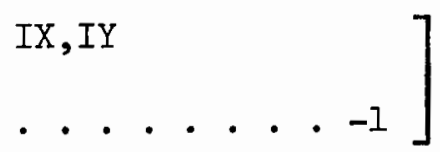

•

•

$-1$

where

IX,IY (integers) are boundary conditions:

$I X=0$ if constrained in global $\mathrm{x}$-direction

$I X=I$ if unconstrained in global $x$-direction

$I Y=0$ if constrained in global $\mathrm{y}$-direction

$I Y=1$ if unconstrained in global $y$-direction

(Note in axisymmetric case radial direction coincides with global $\mathrm{x}$-direction). $\mathbb{N}_{a}, \mathbb{N}_{b}, \mathbb{N}_{c}, \ldots, N_{k}$ (integers) are node numbers of nodes which have boundary conditions IX, IY of the previous line. A - I after $\mathrm{N}_{\mathrm{k}}$ indicates the end of the line. A data subgroup such as (*) above corresponds to each set of boundary conditions IX, IY. Only one line of data $N_{a}, N_{b}, N_{c}, \ldots N_{k},-I$ corresponds to the boundary conditions IX, IY immediately preceeding it, and must be terminated by -1 . If there are too many nodes for one line of data these must be broken into two or more subgroups (*), each having the same boundary conditions IX,IY. Totally unconstrained nodes need not appear in the boundary conditions. A line containing only -1 indicates the end of the boundary conditions. 


\section{(vi) Loading Program}

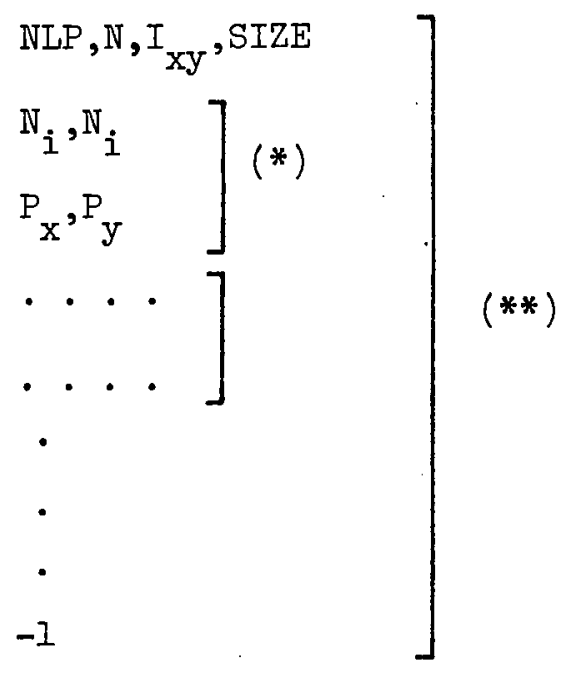

where

NLP (integer) sequential number of the proportional load path (**)

N (integer) node number at which load is to be checked to indicate end of load path NLP

$I_{x y}$ (integer) coordinate direction in which load at node $\mathbb{N}$ is to be

checked: $I_{x y}=1$ for load check in $x$-direction;

$I_{x y}=2$ for load check in $y$-direction

SIZE (real) l, load at node $N$ (in coordinate direction $I_{x y}$ ), at which load path NLP is to be terminated

$\mathbb{N}_{i}, N_{i}$ (integer) is the number of node, repeated, which carries external loads $\mathrm{P}_{\mathrm{x}}, \mathrm{P}_{\mathrm{y}}$ applied in the global $\mathrm{x}, \mathrm{y}$. directions. Nodes which have no external loads need not appear in the loading program. A nodal load subgroup such as (*) above corresponds to each node which has any external loading for load path NLP. Should consecutive node numbers $N_{i}, N_{i+1}, \ldots, N_{k}$ have the same loads $P_{x}, P_{y}$ these can be input as

$\left.\begin{array}{l}\mathrm{N}_{\mathrm{i}}, \mathrm{N}_{\mathrm{k}} \\ \mathrm{P}_{\mathrm{x}}, \mathrm{P}_{\mathrm{y}}\end{array}\right]$.

A line containing only -1 indicates the end of the load path NLP. A loading data subgroup (**) must be entered for each segment of the piecewise linear proportional load path. The end of all load path subgroups is indicated by a line containing only -1 . 


\section{(vii) Output Requested}

$$
\left.\begin{array}{l}
\text { PRINT } \\
\operatorname{IE}_{a},{I E_{b}}_{b} I_{c}, \ldots,{I E_{k}}_{k}-1 \\
N_{a}, N_{b}, N_{c}, \ldots, N_{k},-1 \\
\text { IOUT }_{a}, \text { IOUT }_{b}, \text { IOUT }_{c}, \ldots, \text { IOUT }_{k},-2
\end{array}\right]
$$

where

PRINT

if results are to be given at every node and for all

elements, enter ALL. If results are desired only

at certain nodes and elements, enter SOME. If

ALI is used the two lines of data following are omitted.

If SOME is used:

$\mathrm{IE}_{i}$ (integer) are elements for which results are to be printed

$\mathbb{N}_{i}$ (integer) are nodes for which results are to be printed

Only one line of each is permitted, and the end of each line indicated by -1 .

IOUT $_{i}$ (integer) are the numbers of load increments for which results are to be printed. If IOUT $_{\mathrm{k}}=-1$ the analysis will be terminated after IOUT $_{\mathrm{k}-1}$ load increments. The end of the line is indicated by -2 . In addition results are printed after termination of each proportional load path.

\section{A.3.3 Example of Data and Results for EPTCS}

The pages immediately following list data and results corresponding to a triangular constant strain finite element analysis of the deep cantilever in plane stress described in section 6.3 . The element mesh consisting of 97 nodes and 158 elements is shown in Figure 6.3.2. The result of this analysis is listed in Table 6.3.1. 

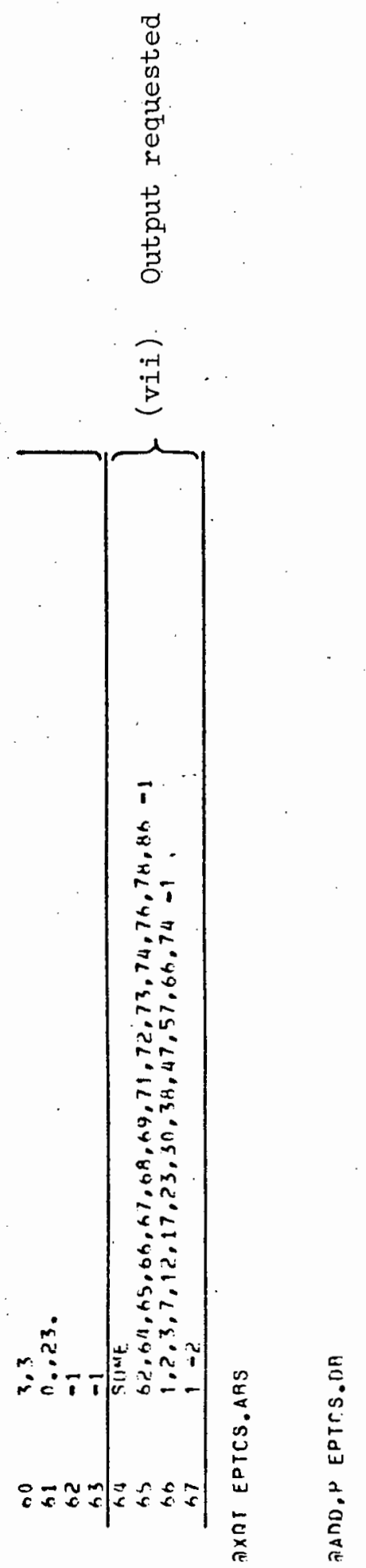
NIMFRICAL ANALYSIS ISTNG THIANGHLAR CONSTANT STPAIN FINITF FLEMENTS

pl.ane sthess, plane sthain OF axisymmetric PKOGPaM.

PLANE STKFSS ANALYSISS

YON HTSFS YIELO CONOITION

FLASTIC, OEPFFCTLY PLASTIC: ANALYSIS TERMINATFO WHEN LOAD INCREMENT MAGNITUNE DECREASES TO. DOS OF TOTAL LOAD HLEMENTS WITH VON MLSES ENUTVALFNT STRESS WITHIN .995 OF YIFLN STRESS TREATFD AS PLASTIC ELEMENTS

UNITS: MN KG

StIRATF AVATLABIE:

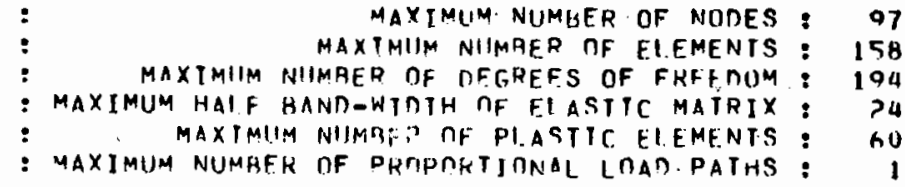

STMRATE RFGUIRED NUMAFH OF NUDES : 9.7 NIIFIF? OF FLEMENTS: $15 \%$ NIIMREL OF OFGREFS UF FHFENOM : 194 HALF GAND-WTRIH HF ELASTIC MATRIX: 194

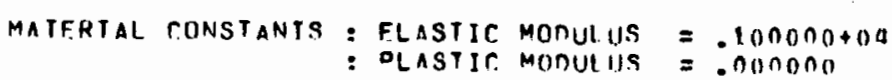
- Puissoas RalTo = . nonono

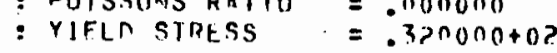

PLATE THITKNESS : $: 100000+01$

COORDJHATFS OF MUNES

\begin{tabular}{|c|c|c|}
\hline & $x$ & $y$ \\
\hline 1 & $.32000+03$ & $.80000+02$ \\
\hline q & $\Rightarrow$ PAnOn+n 3 & . tosnonotnz \\
\hline 9 & $.7500 n+n 3$ & . xsnonona \\
\hline 13 & . Pronon & annolond \\
\hline 17 & . $P$ Pnon+ns & . Onoun \\
\hline$? 1$ & $.70 n 0 n+03$ & (40) non $+n z$ \\
\hline 75 & - inninotns & $.70 n 0 n+02$ \\
\hline 74 & $-18 n \ln +33$ & $\therefore \operatorname{lons} 9+\cap 2$ \\
\hline 23 & $.10^{2} 37+13$ & ? $70 n 00 n+n$ \\
\hline 37 & $.16425+03$ & $n u n+n z$ \\
\hline
\end{tabular}

\begin{tabular}{|c|c|c|}
\hline NONE & $x$ & Y \\
\hline & . $32000+03$ & $.40000+0 ?$ \\
\hline h & . ?AOחO+O3 & . anonoto? \\
\hline 10 & $.250 \cap 0+03$ & $.450 n 0+0 ?$ \\
\hline 14 & . 2 Pก०0+03 & .650 O $0+0 ?$ \\
\hline 19 & 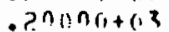 & AnO $\cap 0+0>$ \\
\hline ?? & . $P \cap 0 \cap 0+03$ & $.2 n y \cap 0+0 ?$ \\
\hline $2 h$ & 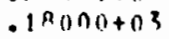 & . $\operatorname{nnn\cap o+0?}$ \\
\hline $3 n$ & Iannotor & : onono \\
\hline 34 & $.16474+113$ & כ) \\
\hline$\{R$ & $.18 \operatorname{sog} 002$ & . ononu \\
\hline
\end{tabular}

\begin{tabular}{|c|c|c|}
\hline$F$ & & \\
\hline 3 & $.32000+0_{3}$ & .00000 \\
\hline & . $28 \cap 0 n+03$ & nonon \\
\hline 11 & $.2 \sin 0 n+03$ & $.25000+12$ \\
\hline 15 & 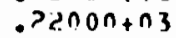 & $45 n 00+02$ \\
\hline 19 & $0000+03$ & $.70000+n 2$ \\
\hline$?$ & . $2000 n+n 3$ & . nonon \\
\hline & $1 B \cap O C+n 3$ & $.5000 n+0$ \\
\hline & $.1700 n+n 3$ & Ronun $n 2$ \\
\hline & $16 A_{1} ?+03$ & $.50000+02$ \\
\hline & $50 n+03$ & \\
\hline
\end{tabular}

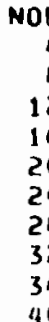

DE
A
$1 ?$
16
$2 n$
24
$2 A$
$3 ?$
$3 h$
$4 n$

$\begin{array}{cc}x & y \\ .28000+03 & .80000+02 \\ .25000+03 & .80000+02 \\ .25000+03 & .00000 \\ .22000+03 & .25000+02 \\ .20000+03 & .55000+02 \\ .18000+03 & .80000+02 \\ .18000+03 & .35000+02 \\ .16969+03 & .75000+02 \\ .16719+03 & .35000+0 ? \\ .16400+03 & .75000+02\end{array}$




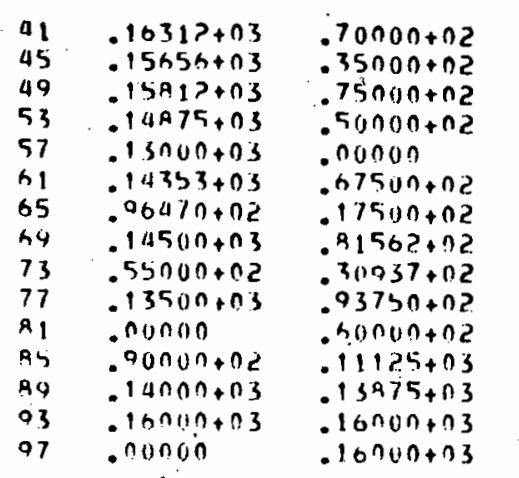

\begin{tabular}{|c|c|c|}
\hline $4 ?$ & $-16219+03$ & $.65000+0 ?$ \\
\hline $4 h$ & $.15375+03$ & $-20000+02$ \\
\hline $5 n$ & $.15625+03$ & $.70000+0 ?$ \\
\hline 54 & $.14500+03$ & $.400 \cap 0+0 ?$ \\
\hline 59 & - 16onotos & $.850 \cap 0+0 ?$ \\
\hline $6 ?$ & $.1^{3} 647+03$ & . $n \cap 000+02$ \\
\hline 66 & $. R \cap O \cap 0+0 ?$ & .00000 \\
\hline 70 & $.130 n 0+03$ & $.731>5+02$ \\
\hline 74 & .00000 & .00000 \\
\hline $7 \mathrm{~A}$ & $.11500+03$ & $.89750+02$ \\
\hline \&? & $.160 \cap 0+03$ & $.1>0 n 0+03$ \\
\hline 86 & $.5 n 0 n 0+(1)$ ? & $.106 ? 5+03$ \\
\hline 90 & $.11000+03$ & $.13687+03$ \\
\hline 44 & $.14000+03$ & $.1600 u+03$ \\
\hline
\end{tabular}

\begin{tabular}{|c|c|c|}
\hline 43 & $.16018+03$ & $.57500+02$ \\
\hline 7 & $-15000+03$ & .00000 \\
\hline & $.15437+03$ & $.65000+02$ \\
\hline 55 & $.14125+03$ & $30000+02$ \\
\hline 59 & $.1552^{\circ}+03$ & . AONUOD+02 \\
\hline 63 & $.12706+n 3$ & $.50 n 0 n+02$ \\
\hline 67 & $.16000+03$ & $.90000+02$ \\
\hline 71 & $.11000+03$ & $.61875+02$ \\
\hline 75 & $.10000+103$ & $.10000+n 3$ \\
\hline 79 & $.85 n 00+n 2$ & \&125n+n2 \\
\hline A3 & $.1400 n+n 3$ & $\therefore 11750+03$ \\
\hline 87 & . nonon & $.10000+n 3$ \\
\hline 91 & . h0non+n? & $.13775+03$ \\
\hline & $.11 n 00+03$ & $.16000+03$ \\
\hline
\end{tabular}

$\begin{array}{lll}44 & .15891+03 & .47500+02 \\ 48 & .16000+03 & .80000+02 \\ 52 & .15156+03 & .57500+02 \\ 56 & .13656+03 & .17500+0 ? \\ 60 & .15054+03 & .75000+07 \\ 60 & : 11294+03 & .35000+02 \\ 68 & .15500+03 & .87187+02 \\ 72 & .85000+0 ? & .47812+0 ? \\ 76 & .15000+03 & .97500+02 \\ 80 & .45000+02 & .71250+02 \\ 84 & .12000+03 & .11500+03 \\ 88 & .160000+03 & .14000+03 \\ 42 & .00000 & .13000+03 \\ 96 & .60000+02 & .16000+03\end{array}$

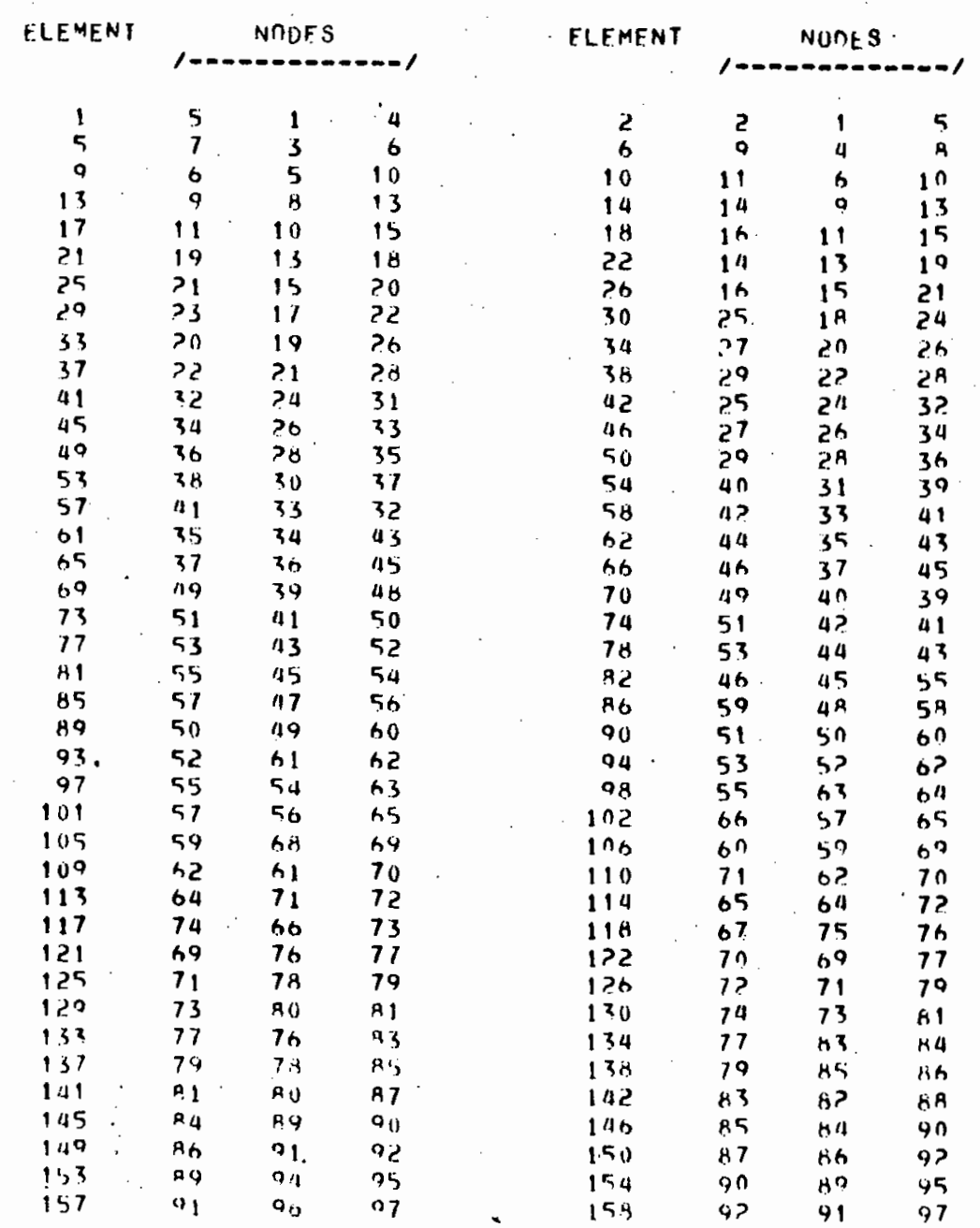

\begin{tabular}{|c|c|c|c|}
\hline ELEMENT & & NODE & \\
\hline 3 & 6 & 2 & \\
\hline 7 & 9 & 5 & 4 \\
\hline 11 & 7 & 6 & 11 \\
\hline 15 & 10 & 9 & 14 \\
\hline 19 & i2 & 11 & 10 \\
\hline 23 & 20 & 14 & 19 \\
\hline 27 & ?.2 & 16 & 21 \\
\hline 31 & 25 & 19 & 18 \\
\hline 35 & 21 & 20 & 27 \\
\hline 37 & 73 & 22 & 29 \\
\hline 43 & 33 & 25 & 32 \\
\hline 47 & 35 & $? 7$ & 34 \\
\hline 59 & 37 & 29 & 36 \\
\hline 55 & 110 & 32 & $3 t$ \\
\hline 50 & 34 & 33 & 42 \\
\hline 63 & 36 & 35 & 44 \\
\hline 67 & 38 & 37 & 46 \\
\hline 71 & 50 & 40 & 19 \\
\hline 75 & 52 & 42 & 51 \\
\hline $70^{\circ}$ & 54 & 44 & 53 \\
\hline 83 & 56 & 46 & 55 \\
\hline 87 & 49 & 48 & 59 \\
\hline 41 & 51 & 60 & 61 \\
\hline 95 & n3 & 53 & क2 \\
\hline 49 & 56 & 55 & 64 \\
\hline 103 & 58 & 67 & 68 \\
\hline 107 & 61 & 60 & 69 \\
\hline 111 & 63 & 62 & 71 \\
\hline 115 & 65 & 72 & 7.3 \\
\hline 119 & 68 & 67 & 76 \\
\hline 123 & 70 & 77 & 78 \\
\hline 127 & 72 & 79 & 80 \\
\hline 131 & 76 & 75 & 82 \\
\hline 135 & 78 & 77 & 84 \\
\hline 130 & $A_{0}$ & 79 & $B_{0}$ \\
\hline 143 & As & $B A$ & 89 \\
\hline 147 & AS & 90 & 91 \\
\hline 151 & AB & 93 & 94 \\
\hline 155 & 90 & 95 & 96 \\
\hline
\end{tabular}




\section{ำ ๑ง}

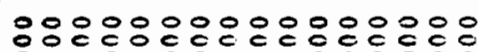

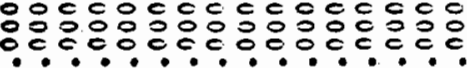

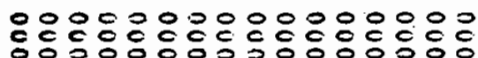

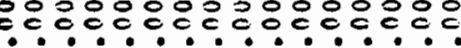

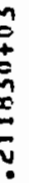

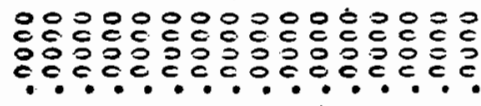

2
2
2
5
5
4
2
0
0
$\vdots$
2
2

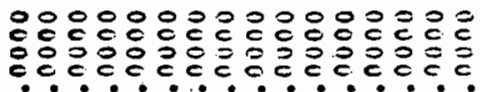

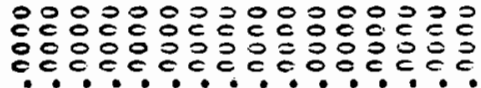

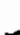

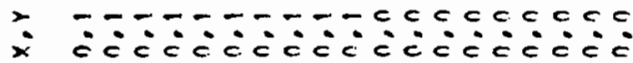

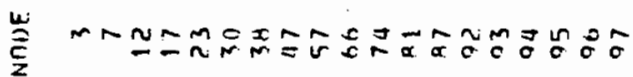

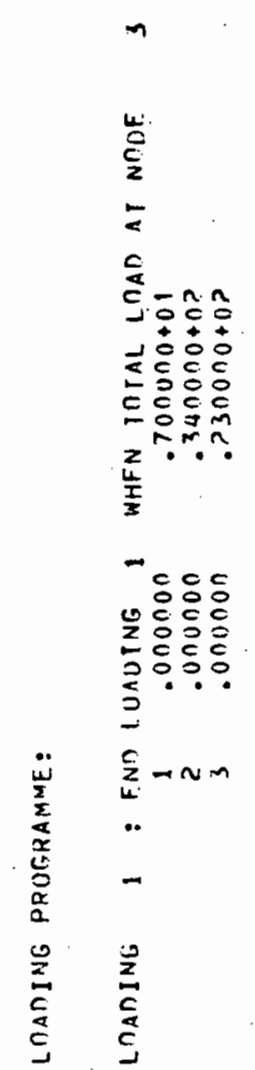

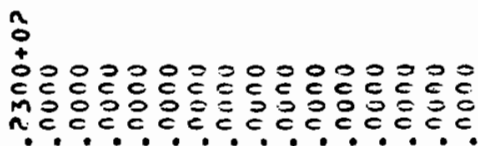

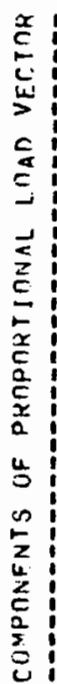

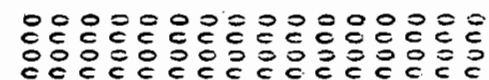

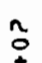

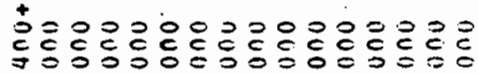
J.5. c.5.

$\overline{0}$

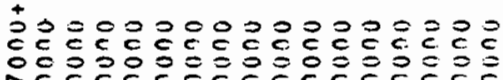

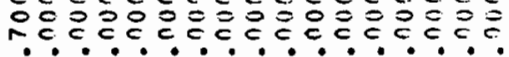

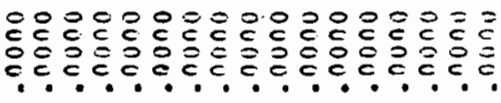


NFXT FLFMFNTS IN INNERGN PLASTIC DEFORMATION : ho

AT LOAD FACTOR . 27HA73+01

LOAD VETIOR, 1 : CIJMIJLATIVE LOAT FACTIDR = .?7A873+01

\begin{tabular}{|c|c|c|c|c|c|c|c|c|c|c|c|}
\hline ELEME & & SIRAIN & $\begin{array}{l}\text { CPEMENTS } \\
\text { PLASTIO }\end{array}$ & LAMAOA & $\begin{array}{l}\text { STRESS } \\
\text { INCREMENT }\end{array}$ & ELASTIC & $\begin{array}{l}\text { STRATNS } \\
\text { PLASTIC }\end{array}$ & TOTAL & STRESS & $\begin{array}{l}\text { YIELD } \\
\text { FUNCTION }\end{array}$ & $\begin{array}{l}\text { STRFSS } \\
\text { CORRECTION } \\
\text { FACTOR }\end{array}$ \\
\hline 6? & $\begin{array}{r}x \\
y \\
x y\end{array}$ & $\begin{array}{r}-.54277-07 \\
-.30272-07 \\
.44790-07\end{array}$ & $=$ & -- & $\begin{array}{l}=.54277+n 1 \\
-.39237+n 1 \\
. ? 2393+n 1\end{array}$ & $\begin{array}{r}-.511277-0 ? \\
-.30232-0 ? \\
.411796-0 ?\end{array}$ & $\begin{array}{l}.00000 \\
.00 n 00 \\
.00 n 00\end{array}$ & $\begin{array}{r}=.54277-02 \\
=.39232-02 \\
.44796-02\end{array}$ & $\begin{array}{r}-.54277+01 \\
=.39732+01 \\
.22343+01\end{array}$ & $-.98540+03$ & $\ldots$ \\
\hline 64 & $\begin{array}{r}x \\
y \\
x y\end{array}$ & $\begin{array}{r}-.331 A 1-02 \\
-.28457-02 \\
.30536-0 ?\end{array}$ & $=-$ & -- & $\begin{array}{r}-33187+01 \\
=.28957+01 \\
.15762+01\end{array}$ & $\begin{array}{r}-.33181=0 ? \\
-.2 A 851=0 ? \\
.30536=0 ?\end{array}$ & $\begin{array}{l}\text {.nonon } \\
\text { nonon } \\
\text {.nonon }\end{array}$ & $\begin{array}{r}-.33187-02 \\
-.29857-02 \\
.30536-0 ?\end{array}$ & $\begin{array}{r}-.33187+01 \\
-.28457+01 \\
.15768+01\end{array}$ & $-.10072+04$ & - \\
\hline 65 & $\begin{array}{r}x \\
y \\
x y\end{array}$ & $\begin{array}{r}-.33191-07 \\
-.15653-0 ? \\
.18102-07 ?\end{array}$ & $=-$ & -- & $\begin{array}{l}-.33187+01 \\
=.15653+01 \\
.90508+00\end{array}$ & $\begin{array}{r}-.33197-07 \\
-.15653-0 ? \\
.19102-0 ?\end{array}$ & $\begin{array}{l}\text { nonon } \\
\text { ononon } \\
\text { ononon }\end{array}$ & $\begin{array}{l}-.33197-0 ? \\
-.15653-0 ? \\
.11102-0 ?\end{array}$ & $\begin{array}{r}-.33187+01 \\
-.15653+01 \\
.90508+00\end{array}$ & $-.10133+04$ & $-\infty$ \\
\hline 66 & $\begin{array}{r}y \\
y \\
X Y\end{array}$ & $\begin{array}{r}-17970=0 ? \\
-.18134-0 ? \\
.211083-0 ?\end{array}$ & $\begin{array}{l}-- \\
--\end{array}$ & -- & $\begin{array}{l}-1797 h+n 1 \\
-18134+n 1 \\
.12048+01\end{array}$ & $\begin{array}{r}-117976-0 ? \\
-.18134=0 ? \\
.27 \cup R 3-02\end{array}$ & $\begin{array}{l}.00 n 0 n \\
\text { nonon } \\
\text { nonon }\end{array}$ & $\begin{array}{r}-.17976-0 ? \\
-.19134-02 \\
.24083-02\end{array}$ & $\begin{array}{r}=1.7976+01 \\
=18134+01 \\
-12042+01\end{array}$ & $-.10164+04$ & $=$ \\
\hline 67 & $\begin{array}{r}x \\
y \\
X Y\end{array}$ & $\begin{array}{r}-.17976-02 \\
-.53524=03 \\
.13205-07\end{array}$ & $\begin{array}{l}= \\
--\end{array}$ & -- & $\begin{array}{r}-17976+101 \\
-53529+100 \\
.66027+100\end{array}$ & $\begin{array}{r}-17476-0 ? \\
-.53529-03 \\
.13205-0 ?\end{array}$ & $\begin{array}{l}\text { nononon } \\
\text { ononon } \\
.00 n 00\end{array}$ & $\begin{array}{r}-.17976-02 \\
-.53529-03 \\
.13205-02\end{array}$ & $\begin{array}{r}=\square 1976+01 \\
=.53527+00 \\
-66 \cap 27+00\end{array}$ & $-.10201+04$ & $\cdots$ \\
\hline $6 A$ & $\begin{array}{r}x \\
y \\
X Y\end{array}$ & $\begin{array}{l}.00000 \\
-.76994-03 \\
.10147-02\end{array}$ & $\begin{array}{l}\ddot{-} \\
--\end{array}$ & -- & $\begin{array}{l}.00000 \\
-.76984+00 \\
.95736+00\end{array}$ & $\begin{array}{r}.00000 \\
\therefore .76 Y 94-03 \\
.19197-0 ?\end{array}$ & $\begin{array}{l}\text { nonun } \\
\text { nonon } \\
\text { noson }\end{array}$ & $\begin{array}{l}.00000 \\
-.76984-03 \\
.19147-02\end{array}$ & $\begin{aligned} .00000 \\
-.76984+00 \\
.95736+00\end{aligned}$ & $-.10207+04$ & $\Rightarrow$ \\
\hline n9 & $\begin{array}{r}x \\
y \\
x y\end{array}$ & $\begin{array}{r}-.26343-01 \\
-.13237-01 \\
.25911-01\end{array}$ & $\begin{array}{l}.00 \text { onon } \\
\text { ononon } \\
\text { nononon }\end{array}$ & .00000 & $\begin{array}{l}=26343+0 ? \\
=13737+02 \\
-12456+02\end{array}$ & $\begin{array}{r}-.26345-01 \\
-.13237-01 \\
.25911-01\end{array}$ & $\begin{array}{l}.00000 \\
.00000 \\
.00000\end{array}$ & $\begin{array}{r}-.263 / 13-01 \\
-.13231=01 \\
.25411-01\end{array}$ & $\begin{array}{r}=.96343+02 \\
\because 13737+02 \\
.12956+n 2\end{array}$ & $-.45776-04$ & .00000 \\
\hline 71 & $\begin{array}{r}X \\
y \\
X Y\end{array}$ & $\begin{array}{r}-.15094-01 \\
-.95374-117 \\
.12864-01\end{array}$ & $\begin{array}{l}=- \\
=-\end{array}$ & -- & $\begin{array}{r}-.15091+02 \\
-.95329+01 \\
.64346+01\end{array}$ & $\begin{array}{l}=.15094-01 \\
=.95324-0 ? \\
.12869-01\end{array}$ & $\begin{array}{l}.00000 \\
.00000 \\
.00000\end{array}$ & $\begin{array}{r}-.15094-01 \\
-.9532 \mathrm{H}-02 \\
.12869-01\end{array}$ & $\begin{array}{r}=.15094+02 \\
=.95328+01 \\
.64346+01\end{array}$ & $-.72498+03$ & - \\
\hline $7 ?$ & $\begin{array}{r}x \\
Y \\
X Y\end{array}$ & $\begin{array}{r}-.10960-01 \\
-.48652-02 \\
.34234-02\end{array}$ & $=$ & -- & $\begin{array}{r}-1096 n+02 \\
-.118657+01 \\
-17117+01\end{array}$ & $\begin{array}{r}-.10960-01 \\
-.49652-02 \\
.34234-02\end{array}$ & $\begin{array}{l}.00000 \\
.00 n 00 \\
.00000\end{array}$ & $\begin{array}{r}-.10940-01 \\
-.48652-02 \\
.34234-02\end{array}$ & $\begin{array}{l}-.10900+02 \\
-.48657+01 \\
-17117+01\end{array}$ & $-.92474+03$ & $\infty$ \\
\hline 73 & $\begin{array}{r}Y \\
Y \\
X Y\end{array}$ & 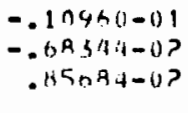 & $\begin{array}{l}-- \\
--\end{array}$ & - & $\begin{array}{r}-11090 n+n 2 \\
=4424 n+n 1 \\
-42^{n} 47+n 1\end{array}$ & $\begin{array}{l}-.10940-01 \\
-.0 R 3014-0 ? \\
. H 50 R A-0 ?\end{array}$ & $\begin{array}{l}\operatorname{non} 00 \\
\operatorname{non} \operatorname{non} \\
\ln \log 0\end{array}$ & $\begin{array}{r}-.10960-01 \\
-.68344-0 ? \\
.85694-0 ?\end{array}$ & $\begin{array}{l}=.1096 n+02 \\
.68344+01 \\
.12842+01\end{array}$ & $-.87701+03$ & $-\infty$ \\
\hline 74 & $\begin{array}{r}x \\
\dot{y} \\
x y\end{array}$ & $\begin{array}{r}-.31 \text { Has }-02 \\
-.162515-02 \\
.211550-07\end{array}$ & $=$ & 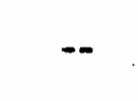 & $\begin{array}{r}-A 1 R H 3+n 1 \\
-46 P 5 n+11 \\
-12775+111\end{array}$ & $\begin{array}{r}-. A 1 B A 3=0 ? \\
-.44250=0 ? \\
.24 S 50-0 ?\end{array}$ & $\begin{array}{l}\text { nonun } \\
\text { nonon } \\
\text { nunun }\end{array}$ & $\begin{array}{l}-.81483-0 ? \\
-.46250-0 ? \\
.24550=0 ?\end{array}$ & $\begin{array}{r}-.81 A 83+01 \\
-.06250+01 \\
.12275+01\end{array}$ & $-.96891+03$ & - \\
\hline
\end{tabular}




\begin{tabular}{|c|c|c|c|c|c|c|c|}
\hline 76 & $\begin{array}{r}x \\
y \\
X y\end{array}$ & $\begin{array}{r}=.64840-0 ? \\
=.43617-07 \\
.18495-02\end{array}$ & $=$ & -- & $\begin{array}{r}=.64940+01 \\
=.43617+01 \\
.92427+00\end{array}$ & $\begin{array}{r}-.64440-0 ? \\
-.43617-0 ? \\
.18485-02\end{array}$ & $\begin{array}{l}.00000 \\
.00000 \\
.00000\end{array}$ \\
\hline 78 & $\begin{array}{r}x \\
v \\
X Y\end{array}$ & $\begin{array}{r}-.52433-0 ? \\
-.37592-0 ? \\
.90272-03\end{array}$ & $\ddot{-\infty}$ & 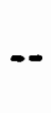 & $\begin{array}{r}-.52433+01 \\
-.32592+01 \\
.44136+00\end{array}$ & $\begin{array}{r}-.521133-02 \\
=.32592-07 \\
.90272-03\end{array}$ & $\begin{array}{l}.0000 n \\
.0000 n \\
.0000 n\end{array}$ \\
\hline 86 & $\begin{array}{r}x \\
y \\
X Y\end{array}$ & $\begin{array}{r}-15 B 31-01 \\
-18978-01 \\
.29452-01\end{array}$ & $=$ & $\cdots$ & $\begin{array}{r}-15831+02 \\
-1897+02 \\
.14731+02\end{array}$ & $\begin{array}{r}=.15 A 31-01 \\
=.19474-01 \\
.29462-01\end{array}$ & $\begin{array}{l}.00000 \\
\text { nonon } \\
\text {. nonon }\end{array}$ \\
\hline
\end{tabular}

$-.64840-02-.64940+01$

$-.43617-02-.43617+01-.98865+03$

1 19485-02 $92427+00$

$-.52433-02-.52433+01$

$-.37592-02-.32597+01-.100 ? 4+04$

$.90272-03 \quad .45136+00$

$-.15831-01-.15931+02$

$-.18978-01-.18979+02$

$.29462-01 \quad .14731+02$

CURRENT PLASTTC ELEMENTS :-

69

\begin{tabular}{|c|c|c|}
\hline NOOF & $n p x$ & DPY \\
\hline 1 & . nonono & $.145211+0 ?$ \\
\hline$?$ & .000000 & $.9 / 168+0 ?$ \\
\hline 3 & . & $.6414 n 7+0 ?$ \\
\hline 7 & $=0000 \cap 0$ & nonuno \\
\hline 1? & . nonono & - nunono \\
\hline 17 & . nonono & .000000 \\
\hline 23 & - nonono & .000000 \\
\hline $3 n$ & .000000 &. noouno \\
\hline 39 & . nunono & . nOnOnO \\
\hline 47 & $.0000 \cap 0$ & . nonono \\
\hline 57 & ט0ח0000 & - nununo \\
\hline 66 & . ח000no & .000000 \\
\hline 74 & nonono & .00 nuno \\
\hline
\end{tabular}

\begin{tabular}{|c|c|}
\hline$P_{x}$ & Py \\
\hline $\begin{array}{l}\text {. nOnOnO } \\
.000000\end{array}$ & $\begin{array}{l}.195211+02 \\
.94 A 16 A+02\end{array}$ \\
\hline .000000 & $.641407+02$ \\
\hline . nonun & .000000 \\
\hline . onnono & . ounono \\
\hline . nonnou & nonono \\
\hline . nonono & conono \\
\hline .000000 & .00 ono \\
\hline . nonano & - nununo \\
\hline . nunono & . nonono \\
\hline . nunono & .nonuno \\
\hline - nunono & .000000 \\
\hline . nunono & .000000 \\
\hline
\end{tabular}

\begin{tabular}{l} 
DISPLACEMFNT INCREMFNT \\
\hline DU
\end{tabular}

nv

$-.179400+01$

.000000

.000000
.000000

. nonuno

inunono

nonono

ounono

. nunono

- nonono

oununo

. 00 nono

nonuno
$.405583+01$

$.106216+01$

$.406216+01$

$.799526+01$

$.225560+01$

$.152839+01$

$.111298+01$

$.864465+100$

$.377537+00$

$.220491+00$

$.366498-01$

Dunooo

\section{TDTAL DISPLACEMFNT}

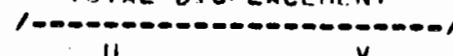

\section{$-.179400+01$}

$.835400+00$

.000000

$.0000 n a$
.000000

.00nuna

- Dunono

- nonuno

- oununa

- ounono

- ongano

.000000

.0000no
$.005583+01$

- $406216+01$ $.406216+01$ . $22560+01$ - $152839+01$ -11120y+01 $.761034+00$ $.546465+00$ $377537+00$ ? $20401+00$ $.366498-01$ .000000

LDAO INTREMFNT B ITERATION 1
ELEMENI SH UNLOADING : LAMBDA

LOAT. INCKFMFNT 15 ITERATION

EIEMENT. BA UNLOADING : LAMARA $=-.959574 .03$

LTAD TNCREHENT IH ITERATIOIY

ELEMENI SA UNLTARING : LAMAOA $=-.178643 .02$

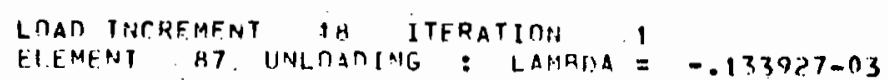

LTAR THSRFHFNT 18 ITERATIIIN?

ELEMENT HT LOANING

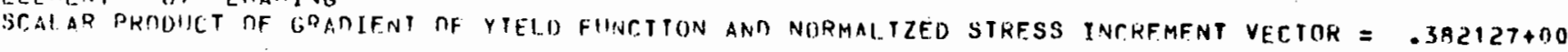

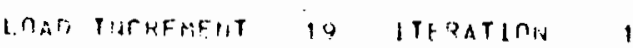


ELEMENT 86 UNLOADING : LAMADA $=-.132379 .02$

LIAD INCHFMFNT ?O ITERATIONA

ELEMENT BS UHLOARING : LAMRDA $=-.961031-03$

IIOAT INTREMENT DI ITERATION

ELEMENT SK UNLOADING : LAMAUA $=-.916677-03$

LOAD THERFMFNT 22 ITERATION

ELEMENT GS UNLOADING: : LAMPDA $=-.113$ ? H5-02

LIAR TNCHEMFNT $>3$ ITERATIONN

ELEMENT HG UNLOADING : LAMADA $=-.544 R I 8-02$

LTAR TNCKFMFNT 33 ITERATION

ELEMENI 87 UNIRADING : LAMPDA $=-.208202-02$

LOAR TNCRFMFNT P3 ITERATION

ELEMENI BR UNLIANING : LAMBOA $=-.136440-0$ ? 
LOAD VECIOR 1 : CUMULATIVE LOAN FACIOR $=.921000+01$;

CURRENT VALUES

\begin{tabular}{|c|c|c|c|c|c|c|c|c|c|c|c|}
\hline \multicolumn{2}{|c|}{ ELEMENT } & $\begin{array}{l}\text { SIRAIN } \\
\text { ELASTIC }\end{array}$ & $\begin{array}{c}\text { INCQEMENIS } \\
\text { PLASTIC }\end{array}$ & \multicolumn{6}{|c|}{ STRAINS } & $\begin{array}{l}\text { YIELD } \\
\text { FUNCTION }\end{array}$ & $\begin{array}{l}\text { STRESS } \\
\text { CORRECTION } \\
\text { FACTOR }\end{array}$ \\
\hline 6? & $\begin{array}{r}x \\
x y\end{array}$ & $\begin{array}{l}-.23677-03 \\
-.12484-03 \\
-.336110=03\end{array}$ & $\begin{array}{r}-.48237-03 \\
-.92725-04 \\
.74770-03\end{array}$ & $.89462-03$ & $\begin{array}{l}=.23677+00 \\
-12444+00 \\
-.16423+00\end{array}$ & $\begin{array}{l}-.3017 \\
-.190 ? \\
.208\end{array}$ & $\begin{array}{r}-.10786-02 \\
-.70950-03 \\
.16994-02\end{array}$ & $\begin{array}{r}-.312 \\
-.19 \\
.275\end{array}$ & $\begin{array}{r}=.30176+02 \\
-19032+02 \\
.10416+02\end{array}$ & $-.30518-04$ & $.9999 a+00$ \\
\hline $6 n$ & $\begin{array}{r}x \\
y \\
X Y\end{array}$ & $\begin{array}{r}-.32611-03 \\
-.26077-03 \\
.29413-13\end{array}$ & $=$ & -- & $\begin{array}{r}-32611+00 \\
-.26027+00 \\
-14287+00\end{array}$ & $\begin{array}{r}-.171 \\
-.153 \\
.163\end{array}$ & $\begin{array}{l}.00000 \\
.00000 \\
.00000\end{array}$ & $\begin{array}{r}-.17189-01 \\
-.15327-01 \\
.16367-01\end{array}$ & $\begin{array}{r}=17189+02 \\
=15327+02 \\
.81 A 34+01\end{array}$ & -.5 & - \\
\hline 65 & $\begin{array}{r}X \\
Y \\
X Y\end{array}$ & $\begin{array}{r}-.37011-03 \\
-.92753-07 \\
.20948-03\end{array}$ & $\begin{array}{l}=- \\
--\end{array}$ & -- & $\begin{array}{r}-32611+00 \\
-.92753-01 \\
.10474+00\end{array}$ & $\begin{array}{r}-.171 A 9-01 \\
-.68113-11 ? \\
-17922-01\end{array}$ & $\begin{array}{l}.00000 \\
.00000 \\
.00000\end{array}$ & $\begin{array}{r}-.171 \times 9=01 \\
-.68113-02 \\
.1 ? 9 ? 2-01\end{array}$ & $\begin{array}{r}-.17189+02 \\
-.68113+01 \\
.64611+01\end{array}$ & $-.67398+03$ & - \\
\hline bh & $\begin{array}{r}x \\
Y \\
X Y\end{array}$ & $\begin{array}{r}=14651-03 \\
=.16692-03 \\
.21043-03\end{array}$ & $=-$ & - & $\begin{array}{l}-11 \\
-.10 \\
.10\end{array}$ & $\begin{array}{r}-.87179-0 ? \\
-.99459-0 ? \\
.13617-01\end{array}$ & $\begin{array}{l}.00000 \\
.00000 \\
.00000\end{array}$ & $\begin{array}{r}-.87179-0 ? \\
-.99959-0 ? \\
.13617-0 ?\end{array}$ & $\begin{array}{r}-.87170+01 \\
-.94959+01 \\
.48086+01\end{array}$ & $-.79727+03$ & - \\
\hline 67 & $\begin{array}{r}X \\
Y \\
X Y\end{array}$ & $\begin{array}{r}-114651-03 \\
-.35345-01 \\
-13932-03\end{array}$ & $=-$ & $-\infty$ & $\begin{array}{r}-14651+00 \\
-.35345-01 \\
.69658-01\end{array}$ & $\begin{array}{r}-.87179-0 ? \\
-.24372-0 ? \\
.97702-0 ?\end{array}$ & $\begin{array}{l}.00000 \\
.0000 n \\
00000\end{array}$ & $\begin{array}{l}-.871 \\
-.243 \\
.927\end{array}$ & $\begin{array}{r}-.87179+n 1 \\
-.24377+01 \\
.46351+01\end{array}$ & $-.89885+03$ & - \\
\hline 68 & $\begin{array}{r}x \\
y \\
X Y\end{array}$ & $\begin{array}{l}.00000 \\
\therefore .68499-01 \\
.16279-03\end{array}$ & $=$ & - & $\begin{aligned} . n 0 n u n \\
-. n B 1199-n 1 \\
.81396-n 1\end{aligned}$ & $\begin{array}{l}.0 \cap 000 \\
-.41518-117 \\
.111>0-01\end{array}$ & $\begin{array}{l}\text { nunon } \\
\text { innion } \\
\text { inonon }\end{array}$ & $\begin{array}{r}.00000 \\
-.41518-02 \\
-11120-01\end{array}$ & $\begin{array}{l}.00000 \\
-.11518+01 \\
.55429+01\end{array}$ & $-.91393+03$ & -- \\
\hline 69 & $\begin{array}{r}x \\
y \\
X Y\end{array}$ & $\begin{array}{l}-.49519-05 \\
-.51213-05 \\
=.17199-04\end{array}$ & $\begin{array}{r}-11067-01 \\
-.26455-n 2 \\
-11297-01\end{array}$ & $.16031-01$ & $\begin{array}{l}-.19919-02 \\
\because .51213-02 \\
-.40544-02\end{array}$ & $\begin{array}{r}-.35379-01 \\
-.23458-01 \\
.16126-01\end{array}$ & $\begin{array}{r}-.50084+00 \\
-.12892+00 \\
.55859+00\end{array}$ & $\begin{array}{r}-.53622+00 \\
-.15238+00 \\
.57472+00\end{array}$ & $\begin{array}{r}-.33162+02 \\
-.21894+02 \\
.75497+01\end{array}$ & $-.45776-04$ & $.10000+01$ \\
\hline 71 & $\begin{array}{r}x \\
y \\
X Y\end{array}$ & $\begin{array}{r}-156.33-014 \\
-12126-04 \\
.26010-04\end{array}$ & $\begin{array}{r}-81933-02 \\
-.18581-02 \\
.79638=02\end{array}$ & $.11576-01$ & $\begin{array}{r}.15683-01 \\
-.12126-01 \\
.13305-01\end{array}$ & $\begin{array}{r}-.33960-01 \\
-.27260-01 \\
.14961-01\end{array}$ & $\begin{array}{r}=30596+00 \\
=.94711-01 \\
.34219+00\end{array}$ & $\begin{array}{r}-.33992+00 \\
-.11197+00 \\
.35715+00\end{array}$ & $\begin{array}{r}-.33415+02 \\
=.71935+02 \\
.73096+01\end{array}$ & $-.30518-04$ & $.10000+01$ \\
\hline $7 ?$ & $\begin{array}{r}X \\
Y \\
X Y\end{array}$ & $\begin{array}{r}-.32457-04 \\
.13417-03 \\
-.17232-03\end{array}$ & $\begin{array}{r}-.5935 n-02 \\
.90032-04 \\
.33754-02\end{array}$ & $.68036-0 ?$ & $\begin{array}{r}-.32457-01 \\
.13417+00 \\
-.61150-01\end{array}$ & $\begin{array}{r}-.35883-01 \\
-.17402-01 \\
.99724-02\end{array}$ & $\begin{array}{r}-18019+00 \\
-14909-01 \\
.83605-01\end{array}$ & $\begin{array}{r}-.21608+00 \\
-.21932-07 \\
.93577-01\end{array}$ & $\begin{array}{r}-.35592+02 \\
-.17284+02 \\
.49537+01\end{array}$ & $-.45776-04$ & $.99998+00$ \\
\hline 73 & $\begin{array}{r}y \\
X Y\end{array}$ & $\begin{array}{r}.2 n 102-44 \\
-.2 n \sin 1-04 \\
-30448-04\end{array}$ & $\begin{array}{l}-.5487 h-n ? \\
-.14747-07 \\
-5407 ?-n ? 2\end{array}$ & $.81705-0 ?$ & $\begin{array}{r}.70100-n 1 \\
-.708111-n 1 \\
.15444-n 1\end{array}$ & $\begin{array}{r}-.33 / 50-01 \\
-.23495-01 \\
-14068-01\end{array}$ & $\begin{array}{r}-18233+00 \\
\because-63 A 27-01 \\
-22047+00\end{array}$ & $\begin{array}{r}-21604+00 \\
-.83322-01 \\
.23564+00\end{array}$ & $\begin{array}{r}-.33349+02 \\
-.22227+02 \\
.72301+n 1\end{array}$ & $-.30518-04$ & $.10000+01$ \\
\hline 74 & $\begin{array}{r}x \\
y \\
X Y\end{array}$ & $\begin{array}{r}-.24192-61 \\
.97263-01 \\
-.10195-13\end{array}$ & $\begin{array}{r}-.42230-02 \\
.51937-04 \\
.19075-08\end{array}$ & $.46719=0 ?$ & $\begin{array}{r}-.2418>-01 \\
.92263-01 \\
-.53523-01\end{array}$ & $\begin{array}{l}=.36188-01 \\
=.17638-01 \\
.14420-0 ?\end{array}$ & $\begin{array}{r}=.00748-01 \\
.90720-02 \\
.30340-01\end{array}$ & $\begin{array}{r}-.132114+00 \\
-.450119-0 ? \\
.41432-01\end{array}$ & $\begin{array}{r}-.35964+02 \\
=.17546+12 \\
.02234+01\end{array}$ & $-.15259-04$ & $.99999+n 0$ \\
\hline
\end{tabular}




\begin{tabular}{|c|c|c|c|c|}
\hline \multirow[b]{2}{*}{$7 \mathrm{~h}$} & 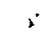 & $-.57490-05$ & $-.23 ? 02-02$ & \multirow[b]{2}{*}{$.24297-6$} \\
\hline & $\begin{array}{l}y \\
x y\end{array}$ & $\begin{array}{l}=.314548-011 \\
=.26334-04\end{array}$ & $\begin{array}{l}.79227-04 \\
.71702-03\end{array}$ & \\
\hline \multirow{3}{*}{78} & $x$ & $-.79533-103$ & -- & \multirow{3}{*}{$-\infty$} \\
\hline & $\gamma$ & $-.130 \cap 9-03$ & -- & \\
\hline & $X Y$ & $-.41034-011$ & -. & \\
\hline \multirow{3}{*}{86} & $x$ & $-.1200 ?-114$ & $\ldots$ & \multirow{3}{*}{$\cdots$} \\
\hline & y & $.0 \cap 13 s-1) 5$ & -- & \\
\hline & $X Y$ & $-00395 b-05$ & -- & \\
\hline
\end{tabular}

CIIRRENT PLASTIC ELEMENIS :-

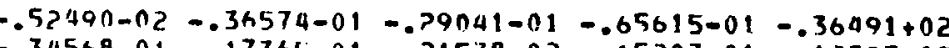

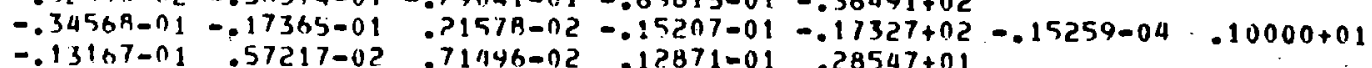

$-.79533+n 0-.34278-01$.nUNU0 $-.311278-01-.3427 R+02$

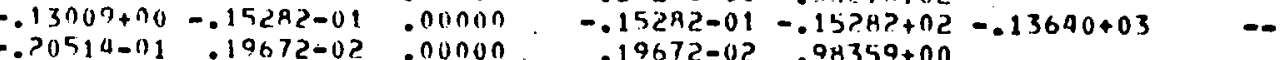

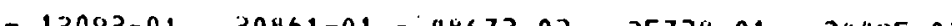

$.60133-02-28123-01-1182011-01-040547-01-.20146+02$

$-.41978-02.27662-01 \quad .35395-01 \quad .59057-01-.08196+02-.19624+02 \ldots$

$\begin{array}{llllll}11 & 47 & 43 & 44 & 45 & 46\end{array}$

118

54

$55 \quad 5 h \quad 57 \quad 58$

5960

$91 \quad 105$

79

H7

$9 n$

\begin{tabular}{|c|c|c|}
\hline TDE & $n P x$ & nPy \\
\hline 1 & . ח0nono & $.423370+01$ \\
\hline ? & . nonט00 & . Pu5o31+01 \\
\hline 3 & .000000 & $.139107+01$ \\
\hline 7 & . nonono & . nunono \\
\hline 1? & .000000 & .000000 \\
\hline 17 & nonono & . 20nogoo \\
\hline 23 & (n) & - nonnouo \\
\hline $3 n$ & nijnun!̣ & - nonuno \\
\hline 38 & . nonunu & .000000 \\
\hline 47 & . nonono & . nonoou \\
\hline 57 & . nunonon & - nonuno \\
\hline 66 & . nonono & .000 Uno \\
\hline $7 \mathrm{t}$ & 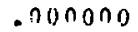 & . nonono \\
\hline
\end{tabular}

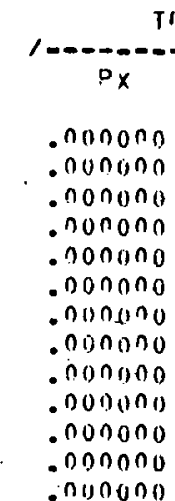

IITAL L MAD

PY

$044700+0 ?$ x.13 $3140+03$ ? $11430+03$ inconor - ninoso

- nonano

nonano

conono

nununa

nununo

000000

.000000

.0000000

\section{DISPLACFMENT INCREMENT}

nu

nv

$-.94 n 761-01$

$\therefore 437291-01$

.000000

. Donuno

ounono

inunona

- nunono

- noouno

inonono

nonono

-0
nonono

ononono
ononono
$.713338+00$

.$>12475+00$

- $113452+00$

$-175078+00$

- Tesoraton

$647978-01$

-

- ?7RQ?4-0

$167080-0$

$.764344-02$

. $651903-03$

- nonano

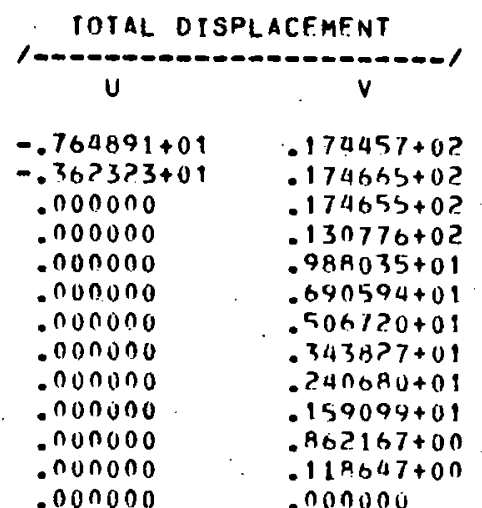




\section{A.4.1 Data Input for EPCQI}

HEADINGI

HEADING2

i) heading statements

TYPE

NN, NE , E , EP , RNU, SZERO ,ETA, IO, NLAM, (THIK) , FRAC

ii) structure statèments

$1, \mathbb{N}_{1}, \mathbb{N}_{2}, \ldots, \mathbb{N}_{12}, \mathrm{NTYPE}$

$\left.\mathrm{X}_{1}, \mathrm{Y}_{1}, \mathrm{X}_{2}, \mathrm{Y}_{2}, \ldots, \mathrm{X}_{\mathrm{k}}, \mathrm{Y}_{\mathrm{k}}.\right]$
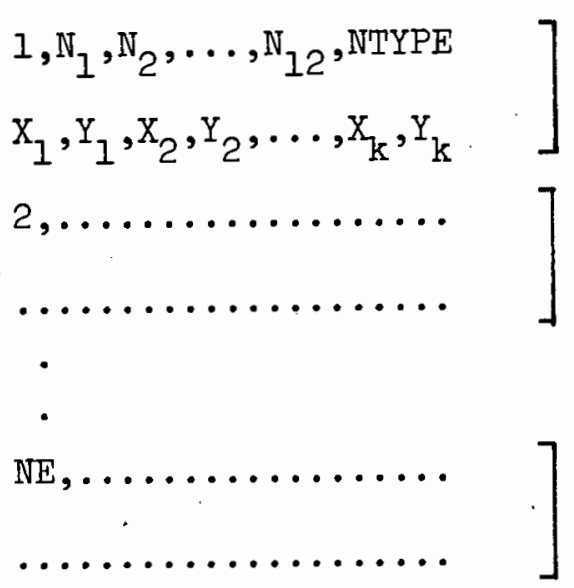

IX, IY

$\mathrm{N}_{\mathrm{a}}, \mathrm{N}_{\mathrm{b}}, \mathrm{N}_{\mathrm{c}}, \ldots, \mathrm{N}_{\mathrm{k}},-\mathrm{I}$

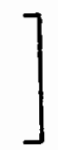

IX, IY

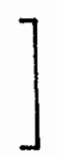
iii) element incidences and nodal coordinates

iv) boundary conditions
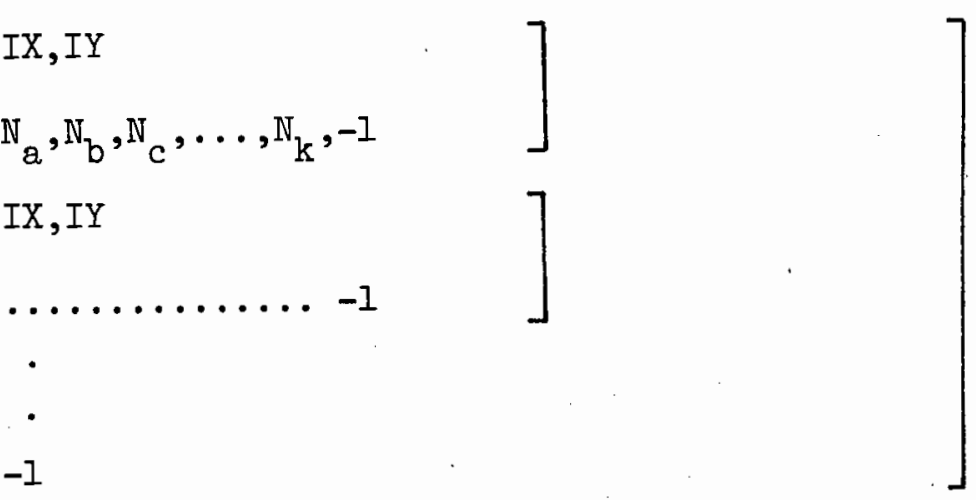

$\mathrm{NLP}, \mathrm{N}, \mathrm{I}_{\mathrm{xy}}, \mathrm{SIZE}$

IE, ISIDE, $\left.\mathrm{T}_{x}, \mathrm{~T}_{y}, \mathrm{NTYPE}\right](*)$

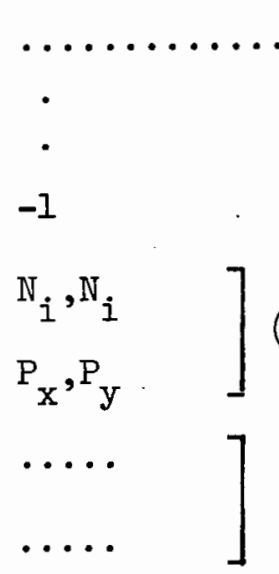

$(* * *)$ (**)

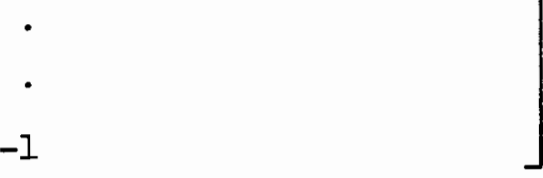

continued 


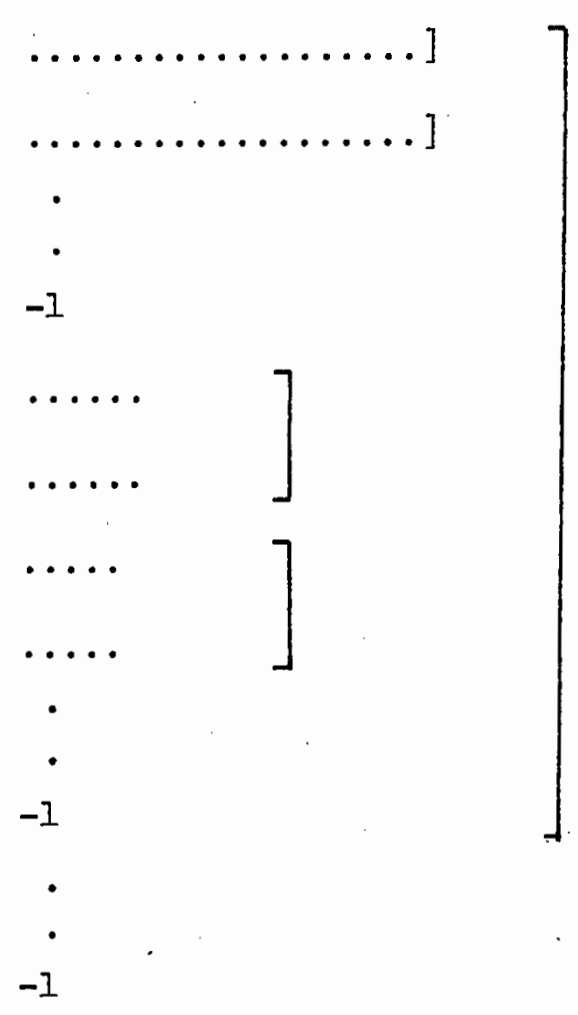

PRINT

$I E_{a}, I G_{a}, I E_{b}, I G_{b}, \ldots, I E_{k}, I G_{k},-i$

$\mathrm{N}_{\mathrm{a}}, \mathrm{N}_{\mathrm{b}}, \mathrm{N}_{\mathrm{c}}, \ldots, \mathrm{N}_{\mathrm{k}},-1$

vi) output requested

vi) output requested
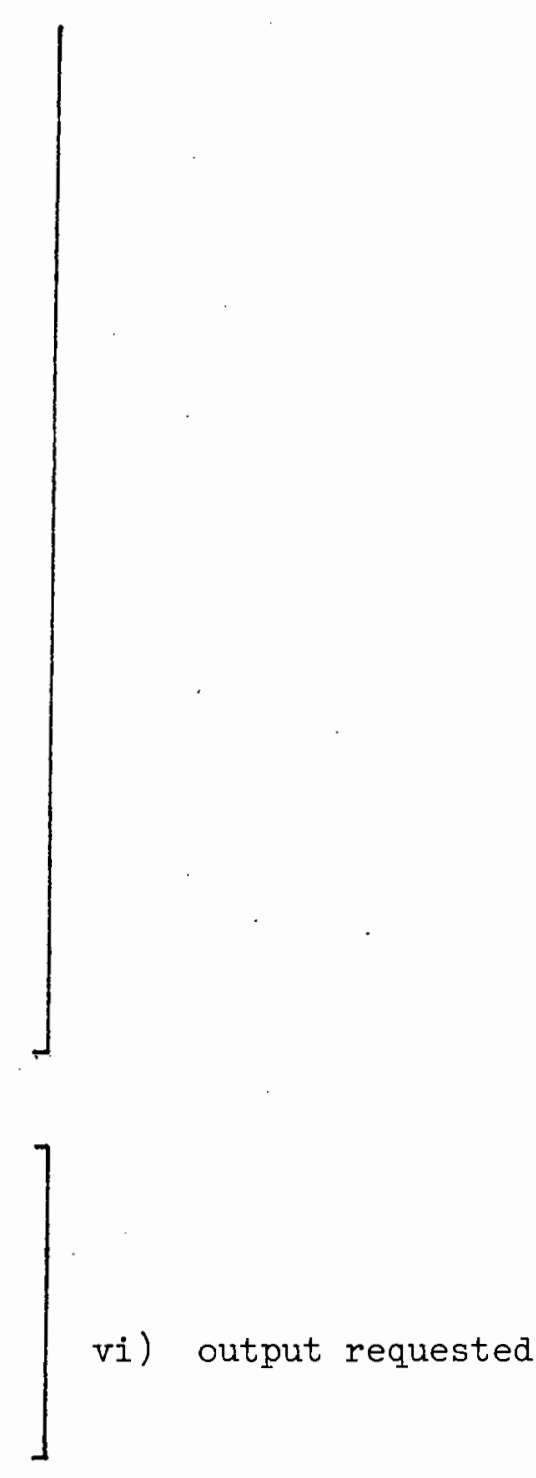

\section{A.4.2 Description of Data Input}

(i) Heading Statements

(as for program EPTCS; see section A.3.2.(i))

\section{(ii) Structure Statements}

TYPE

NN, NE, E , EP , RNU , SZERO, ETA, IO, NLAM, (THIK) , FRAC

(as for program EPTCS; see section A.3.2.(ii)), and where

IO (integer) integration order for Gauss quadrature; maximum value 4 , recommended value 3 . 
NLAM (integer) number of plastic multipliers associated with each element: $\quad$ NLAM $=I$ for one 'average' multiplier per element; or NLAM $=10^{2}$ for one multiplier per integration point; (see section 4.6).

\section{(iii) Element Incidences and Nodal Coordinates}

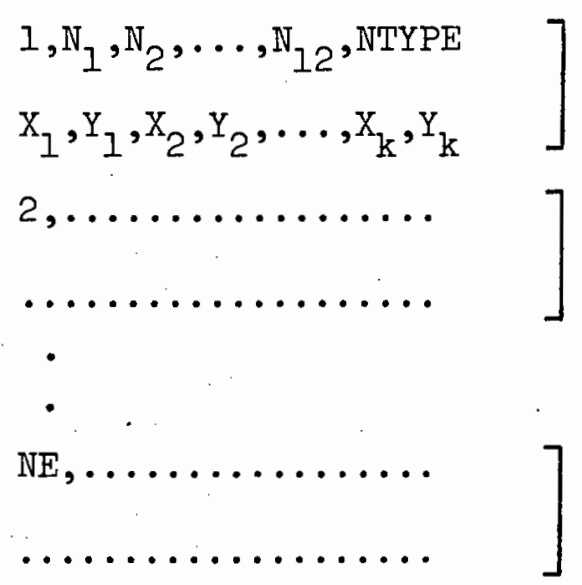

where

$\mathbb{N}_{1}, \mathbb{N}_{2}, \ldots, \mathbb{N}_{12}$ (integer) element incidences (see Figure 4.I)

NTYPE (integer) = $\quad=1$ if boundaries of element are straight;

$\neq I$ if any boundary of element is curved

$\mathrm{X}_{1}, \mathrm{Y}_{1}, \ldots, \mathrm{X}_{\mathrm{k}}, \mathrm{Y}_{\mathrm{k}}$

if NTYPE $=1$ then $\mathrm{k}=4$, i.e. input global coordinates of corner nodes of element only; if NTYPE $\neq 1$ then $\mathrm{k}=12$, i.e. input global coordinates of all nodes of element (see Figure 4.1)

A data subgroup such as (*) above corresponds to each isoparametric element.

(iv) Boundary Conditions

(as for program EPTCS; see section A.3.2.(v)). (page A-9) 


\section{(v) Loading Program}

$\mathrm{NLP}, \mathrm{N}, \mathrm{I}_{\mathrm{xy}}, \mathrm{SIZE}$

(as for program EPTCS; see section A.3.2.(vi)) (page $A-10)$

IE, ISIDE, $\left.T_{x}, T_{y}, N T Y P E\right](*)$

where

IE (integer) number of element

ISIDE (integer) number of element side on which boundary traction $\mathrm{T}_{\mathrm{X}}, \mathrm{T}_{\mathrm{y}}$ acts :

for side $s=+1$ enter 1

for side $r=-1$ enter 2

for side $s=-1$ enter 3

(see Figure 4.1)

for side $r=+1$ enter 4

$\mathrm{T}_{\mathrm{X}}, \mathrm{T}_{\mathrm{y}}$ (real) boundary traction components respectively in global

$\mathrm{x}, \mathrm{y}$ directions: force per unit area acting along

element boundary ISIDE. (for axisymmetric case do not multiply by $2 \pi$ ).

NTYPE (integer) if element boundary ISIDE is straight enter 1;

if element boundary is arc of circle with centre

the origin of global coordinate system, enter 2.

In this case $\mathrm{T}_{x}$ is radius of circular arc, and $\mathrm{T}_{\mathrm{y}}$

is magnitude of radial force per unit area.

(see example of data input, section A.4.3)

A data sybgroup such as (*) above corresponds to each element

side which has non-zero boundary tractions. A line containing only

-I indicates the end of boundary tractions for load path NLP. If

there are no boundary tractions for load path NLP enter -1 .

$\left.\begin{array}{l}\mathbb{N}_{i}, N_{i} \\ P_{x}, P_{y}\end{array}\right] \quad \begin{aligned} & \text { nodal point loading for load path NLP (as for program } \\ & \text { EPTCS, see section } A .3 .2 .(v i) \text { ) }\end{aligned}$


A line containing only -1 indicates the end of nodal point loads for load path NLP. If there are no nodal point loads for load path NLP enter -1 .

A loading data subgroup (***) must be enetered for each segment of the piecewise linear proportional load path. The end of all load path subgroups is indicated by a line containing only -1 .

\section{(vi) Output Requested}

PRINT

$I E_{a}, I G_{a}, I E_{b}, I G_{b}, \ldots, I E_{k}, I G_{k},-I$

$\mathrm{N}_{\mathrm{a}}, \mathrm{N}_{\mathrm{b}}, \mathrm{N}_{\mathrm{c}}, \ldots, \mathrm{N}_{\mathrm{k}},-\mathrm{I}$

IOUT $_{a}$, IOUT $_{b}$, IOUT $_{c}, \ldots$, IOUT $_{k}-2$

(as for program EPTCS; see section A.3.2.(vii), and where

$I E_{i}, I G_{i}$ (integer) are element and integration point numbers for which stresses and strains are to be printed. Integration points are numbered for each element on lines of constant $r$ value, beginning at the Gauss point closest to $(r, s)=(-I,-I)$, and increasing in magnitude in the positive local s coordinate direction. For example third order numerical integration has Gauss points $1,3,7,9$ respectively closest to corners $(r, s)=$ $(-1,-1),(-1,+1),(+1,-1)$ and $(+1,+1)$. (see Figure 4.1)

\section{A.4.3 Example of Data and Results for EPRCS}

The pages immediately following list data and results corresponding to a cubic quadrilateral finite element analysis of the deep beam in plane stress described in section 6.3. The element mesh consisting of 192 nodes and 31 elements is shown in Figure 6.3.2. The result of this analysis is listed in Table 6.3.1. 


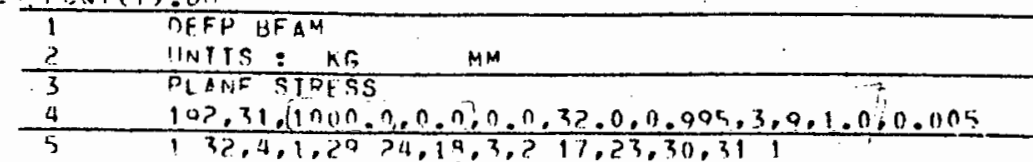

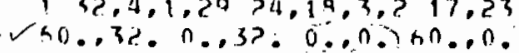

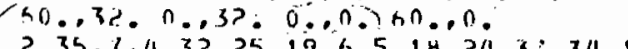

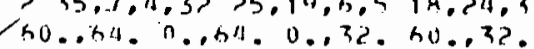

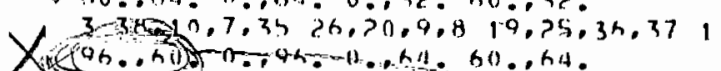

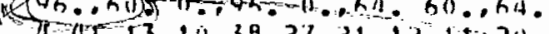

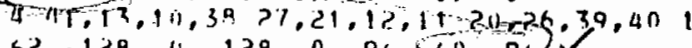

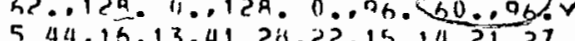

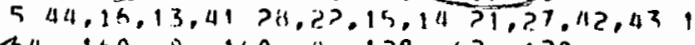

ha

$h$ hol, 3P, PQ, $, 7,52,44,31,3045,51,5 H, 501$

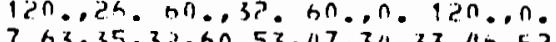

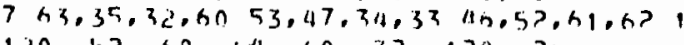

len..

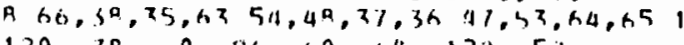

1r ${ }^{2}, 41$ उ

120,11, 3h, Bh 5 ,

12 .

1 th

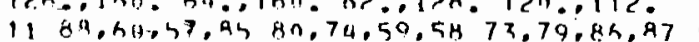

$157.110,23,120,06,120,00.157 .46,000$

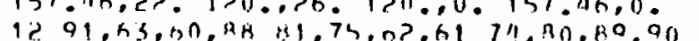

$147.86,44,1>0,052,120.26,151.46,2 ?$

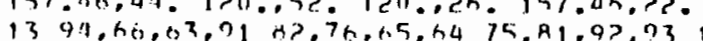

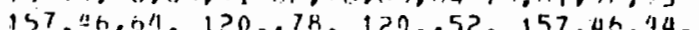

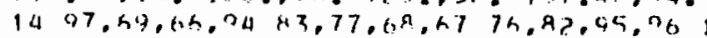

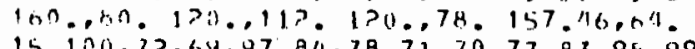

100 , this $126,94,79,71,7 n 77,83,95,90$

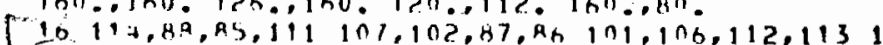

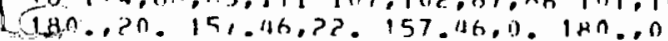

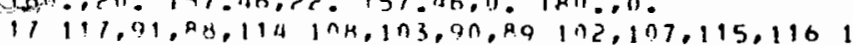
18n..4n, 157.4h.144. 157.4h, ? ? 180..?

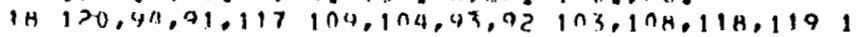

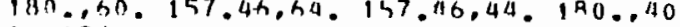

$191 ? 3,47,04,1>0110,105,46,95$ 104.109,1?1.1?2।

18n..,8n. 160..An. 157.4h, h4. 190,.,ho.

30) $137,114,111,134130,135,113,1121>4,1>9,135,1361$

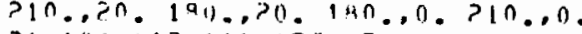

$>1111,117,114,137131,17 \mathrm{~h}, 11 \mathrm{~h}, 113,173,130,134,1391$

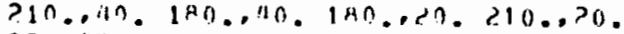

?? 143,1?0,117,140 132,1?7,119,118176,131,141,192 ?.10..6n. 180..ho. 180..4n. $210 \ldots 110$

$>3146,1>3,1>0,143 \quad 134,1>8,1>2,1>1177,132,114,145$

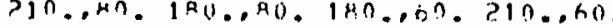

$34160.137 .134 .157 \quad 153.149 .134 .0135147 .152 .154 .159$

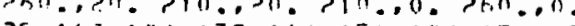

$2516,3,140,137,160154,149,139,134,148,153,161,162$ $26 n \ldots 4 n \cdot 2: 0, \ldots 10,210, .20$. $240, .>0$

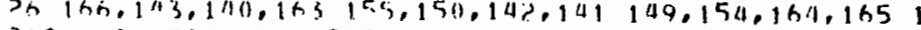

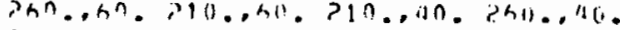

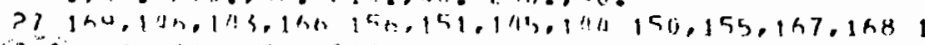

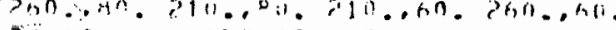

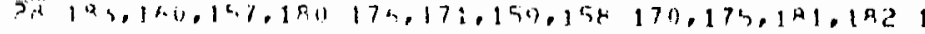

(i) Heading statements

(ii) Structure statements

(iii)

Element incidences and nodal coordinates 


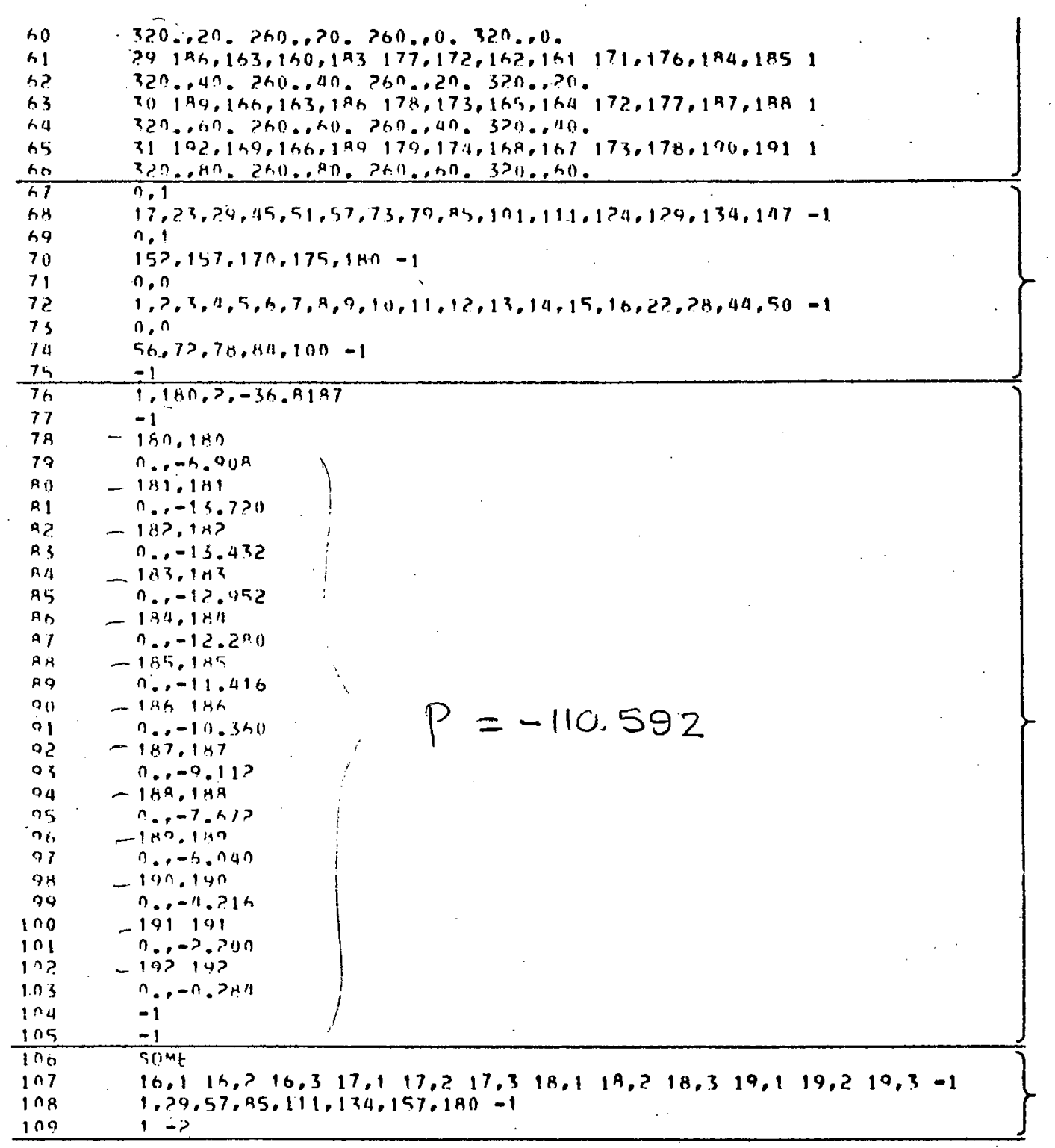

(iv) Boundary conditions

(v) Loading program 
DFEN REAM

NIMFETCAL ANALYSIS ISTHF TWO DIMENSIUNAL TWFLVE NODF ISUPARAMETRTC FINTTF. ELEMENTS

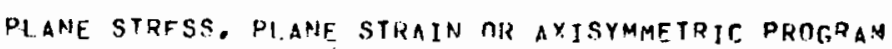

CHATC INTFRPOT.ATION FIINCTTONS

GUARRAITC BTRAIN VARIATTON

NHMEYICAL INTFGPATION USIMG GALISS ULADRATHRE

1)QDFR OF THTERRATTON $=3$

HLANE STKFSS AINALYSIS

VIN MTSFS YIELD CONDITIONN

FLASTIC, PERFECTLY PLASTIR: ANALYSIS TERM INATED WIHEN LOAD INCREMENT MAGNITUNE DECREASFS TO .0O5 OF TOTAL, LOAD

INIEGRAIION POINTS WITH VON MTSFS ERUTVALFNT STRESS WTTHIN . 94G OF YIFLD STRESS TREATFO AS PLASTIC

UNIITS: KG MM

STIJPARE AVATLABIF

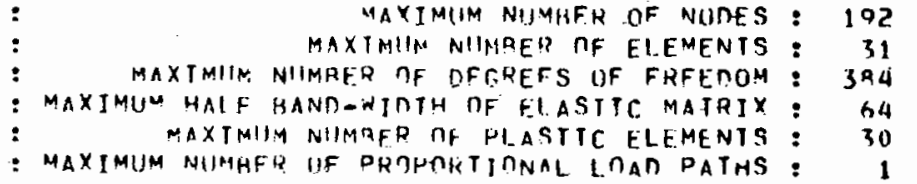

STORATE HFIJIIREA

NUMGER OF NONES : 192 MIMAER DF DFCPFFS OF FUFEDUM

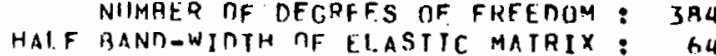

MATERTAI. CONSTANIS : FLASTIR MONULUUS $=.100000+04$

$$
\begin{aligned}
& \text { : Plastir monulus }=. \text { nonono } \\
& \text { : polssovs hatio }=\text {. nonono } \\
& \text { : PIFLO STPESS }=\text {. }
\end{aligned}
$$

PLATE THIRKMESS : . InONOOn+ni

\begin{tabular}{|c|c|c|c|c|c|c|c|c|c|c|c|}
\hline 1 & 10non & nono & $\stackrel{\text { mont: }}{?}$ & $\begin{array}{c}x \\
. \sin \theta \theta^{2}\end{array}$ & $.106 A^{Y} 7+U P$ & $\begin{array}{c}\text { NnOF } \\
3\end{array}$ & $\begin{array}{c}x \\
.00 n u n\end{array}$ & $\begin{array}{c}y \\
.0 .133^{3}+02\end{array}$ & NOחE & $\begin{array}{c}x \\
.00000\end{array}$ & $\begin{array}{c}Y \\
.32000+0 ?\end{array}$ \\
\hline
\end{tabular}

COORDTNAIES UF NUIES 


\begin{tabular}{|c|c|c|c|c|c|c|c|c|c|c|c|}
\hline 5 & .00000 & $.42 h 67+n 2$ & $h$ & $.000 n o$ & $.53333+0 ?$ & 7 & .00000 & $.64000+02$ & 8 & .00000 & $.746667+0 ?$ \\
\hline & - nonon & $.85335+122$ & $1 n$ & . 0nano & $.96000+0 ?$ & 11 & . nonoo & $.10667+n 3$ & 12 & .00000 & $11733+03$ \\
\hline 13 & 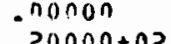 & $-1290 n+n 3$ & 14 & . & $.138 h \gamma+03$ & 15 & .00000 & $.14933+03$ & 16 & .00000 & $160 n 0+03$ \\
\hline 17 & $\begin{array}{r}P 0000+02 \\
207\end{array}$ & - nonno & $1 \mathrm{~A}$ & - ?nonoto? & $-3 P 0 \cap 0+0 ?$ & 19 & $.2000 n+02$ & $.64000+02$ & 20 & $.20000+02$ & $.96000+02$ \\
\hline 31 & 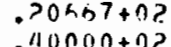 & $-12^{2} 00+03$ & $2 ?$ & $.21333+0 ?$ & $-12000+03$ & 23 & $.4000 n+n 2$ & . nonon & 24 & $.40000+0 ?$ & $.3>0 \cap 0+0 ?$ \\
\hline 29 & ק & - hunonton & $2 h$ & $-4 n 0 n 0+0$ ? & - anonoto? & $? 7$ & $.01333+02$ & $-1280 n+03$ & 28 & $.47667+02$ & 1 . $140 n n+03$ \\
\hline 35 & 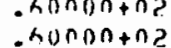 & $\begin{array}{l}0.0406 n \\
02567+02\end{array}$ & $\begin{array}{l}3 n \\
34\end{array}$ & 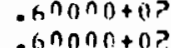 & $\begin{aligned} 10667+07 \\
5333\end{aligned}$ & 31 & בan+anon & $. ? 1333+02$ & 32 & $-60000+0 ?$ & ? $3>0 \cap 0+0$ \\
\hline & 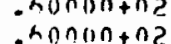 & 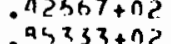 & 38 & - $6 n n n n+0 ?$ ? & $\begin{array}{r}53333+02 \\
0400102\end{array}$ & $\begin{array}{l}35 \\
30\end{array}$ & - $60000 n+n 2$ & $-\operatorname{sannon+n2}$ & 36 & $.60000+0 ?$ & $.711667+0 ?$ \\
\hline 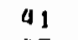 & -hanuntaz & $\because 12 a_{0} 00+03$ & 47 & $: x>6 n>+0$ ? & 0 & 39 & $\begin{array}{r}-60667+02 \\
-3333+02\end{array}$ & $\begin{array}{r}10007+03 \\
14933+03\end{array}$ & 40 & $.01333+02$ & $.11733+03$ \\
\hline 45 & 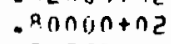 & - nonon & ith & . anonnto? & .3nonoto? & 47 & 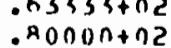 & $\begin{array}{l}.60000+02 \\
.600\end{array}$ & $4 \mathrm{~A}$ & $\begin{array}{l}.04000+0112 \\
-40000+0 ?\end{array}$ & $\because 1$ \\
\hline $\begin{array}{l}49 \\
52\end{array}$ & $-A_{1} 333+n ?$ & $.12>67+n s$ & $5 n$ & . Alanh7+0? & . 1 honnto3 & 51 & $.1000 n+03$ & nonon & 52 & $.10000+03$ & $\begin{array}{l}.90 \\
.28\end{array}$ \\
\hline 53 & $-10 n \operatorname{lon}+n 3$ & $-50000+02$ & 54 & $.10000+03$ & $.84000+0 ?$ & 55 & $.10067+03$ & $.11733+03$ & 56 & $.10533+03$ & 1 honoto3 \\
\hline 57 & $-12 n n n+n 3$ & - nisnon & $5 \mathrm{~A}$ & $.1300_{0}+0^{3}$ & -Anbh7+01 & 59 & $.1200 n+03$ & $.17333+02$ & 60 & $1>0 \cap 0+03$ & $26000+0 ?$ \\
\hline h1 & $-1200 n+03$ & .34 & o? & $.1>000+03$ & $.43333+0 ?$ & 63 & $-120 u 0+03$ & $.52000+02$ & 64 & $12000+03$ & $.6 \cap 667+02$ \\
\hline nh & $-1 \geqslant 0<1 n+03$ & $-04333+02$ & $6 h$ & $-1 ? 0 n n+03$ & . $7 A_{0} \cap 0+0 ?$ & 67 & $.12 n 00+n 3$ & $.89333+02$ & $6 \mathrm{~A}$ & $12000+03$ & $.10067+03$ \\
\hline $\begin{array}{l}29 \\
73\end{array}$ & $\begin{array}{r}-12 n 01 n+n 3 \\
13240+03\end{array}$ & $-1120 n+n 3$ & 70 & $.12200+03$ & $-12400+03$ & 71 & $.12400+03$ & $.14400+03$ & $7 ?$ & $17000+03$ & Ihonotos \\
\hline $\begin{array}{l}73 \\
71\end{array}$ & $\begin{array}{r}13340+03 \\
13333+03\end{array}$ & - non & 74 & $-132114+03$ & .246 & 75 & $.13 ? 49+03$ & $.49333+02$ & 76 & $.13249+03$ & $.73333+0 ?$ \\
\hline $9_{1}$ & $\begin{array}{r}-13363+03 \\
144097+03\end{array}$ & $\begin{array}{l}10933+n 3 \\
010967+n ? 2\end{array}$ & $\begin{array}{l}78 \\
82\end{array}$ & $\begin{array}{r}-13733+02 \\
1007+03\end{array}$ & $-14001)+03$ & 79 & $.14497+03$ &.$n 0$ & 80 & $.14497+03$ & $.23333+02$ \\
\hline 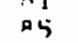 & $\begin{array}{r}19191+03 \\
-1574 h+03\end{array}$ & $\begin{array}{l}\text { - Anghetena } \\
\text { nonon }\end{array}$ & $\begin{array}{l}8 ? \\
86\end{array}$ & $\begin{array}{r}14497+03 \\
15746+03\end{array}$ & 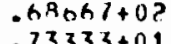 & $\begin{array}{l}83 \\
87\end{array}$ & $-14667+03$ & $.90667+02$ & 84 & $.14867+03$ & $-1+000+03$ \\
\hline RQ & $\begin{array}{r}1574 h+105 \\
.1574 h+13\end{array}$ & $=29333+n 2$ & on & $\begin{array}{r}-157416+03 \\
1574 t a+03\end{array}$ & $=7<3<3+01$ & A7 & $-1574 h+03$ & $.14467+02$ & BA & $-15746+03$ & $.22000+0 ?$ \\
\hline$x_{3}$ & $.1574 h+n 3$ & $\begin{array}{l}\because 57333+n 2 \\
0\end{array}$ & 94 & $\begin{array}{l}.1274 h+03 \\
.1574 h\end{array}$ & 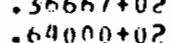 & & $\begin{array}{l}-15746+03 \\
15831+03\end{array}$ & $=44000+02$ & $9 ?$ & $-15746+03$ & - $5 \operatorname{nns} 7+1) 2$ \\
\hline $07>>1$ & $.1600 n+n 3$ & Sijnon+n? & 99 & $: 160 n u+n 3$ & $10067+03$ & 94 & - $15031+n 3$ & $\begin{array}{r}-69333+02 \\
13333+03\end{array}$ & 96 & $-14915+03$ & $.74667+0 ?$ \\
\hline 101 & $.161997+03$ & inunion & $10 ?$ & $.16407+03$ & $.21333+0 ?$ & 103 & $0.16497+03$ & $\begin{array}{r}-13533+03 \\
-42667+02\end{array}$ & 104 & $\begin{array}{r}160000+03 \\
16497+03\end{array}$ & $\begin{array}{l}16000+103 \\
6>601+0 ?\end{array}$ \\
\hline 115 & $.16467+n 3$ & . A & 106 & $.17219+03$ & . nns & 107 & $.17 ?$ & .20 & $10 \mathrm{~A}$ & $.17249+03$ & $.41333+0 ?$ \\
\hline 109 & $-17>110+03$ & & $11 n$ & .17 & . AnO & 111 & .1 Bnon+n3 & .00 & $11 ?$ & $0+03$ & $.666 h 7+01$ \\
\hline $\begin{array}{l}113 \\
117\end{array}$ & $\because 9$ & .13 & 114 & $-19000+03$ & $.2 n c$ & 115 & $.18000+03$ & . ?h667+02 & 116 & $n 0+03$ & $.33333+02$ \\
\hline 1? & 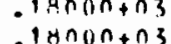 & 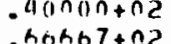 & 2119 & $-12000+03$ & $-46667+0 ?$ & 119 & $-18 n 0 n+03$ & .53 & $12 n$ & $.18000+03$ & $n 0+0 ?$ \\
\hline $1>5$ & 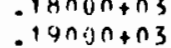 & 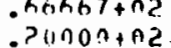 & $12 ?$ & $\begin{array}{l}-1 \ln n n(1)+03 \\
180 n(1) 03\end{array}$ & $.73333+0 ?$ & $1 \geqslant 3$ & $.18 n$ & & 124 & $.19000+03$ & .00000 \\
\hline 174 & 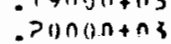 & : nunuon & $13 n$ & $\begin{array}{l}1 \\
3 n_{0} 0 n_{0+0}\end{array}$ & ? & 127 & $\begin{array}{r}19000+03 \\
2000\end{array}$ & - 6000 & 12 & $.19000+03$ & $.90000+02$ \\
\hline 133 & . & -94 & 134 & $2.21000+03$ & ? & 135 & 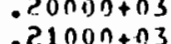 & . 40 no & 13 & & $+0 ?$ \\
\hline 137 &.$>1 n 0 n+n 3$ &.$>0$ & $13 R$ & & $.24647+0 ?$ & 139 & P. $1000+03$ & .83333 & $\begin{array}{l}156 \\
140\end{array}$ & $\begin{array}{r}-21000+03 \\
-21000+03\end{array}$ & it \\
\hline 111 & $.21 \mathrm{n}$ & & 14? & .21 & .53 & 143 &.$>100 n+03$ & Sononotoz & 144 & $.21000+03$ & $.66667+02$ \\
\hline &. $\ln \theta$ & & $14 \mathrm{~h}$ & & .hit & 147 & ג & & 148 & $.27647+03$ & $.20000+02$ \\
\hline 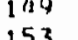 & $\begin{array}{r}-225,67 \\
24202\end{array}$ & -40 & $15 n$ &.$?$ & - h & 151 & &. & 1 & .2433 & .00000 \\
\hline $\begin{array}{l}153 \\
157\end{array}$ & $\begin{array}{l}.343,33 \\
36 n 00\end{array}$ & .000 & 154 & & & 1 & & & & & \\
\hline int & 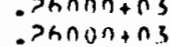 & 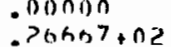 & $\begin{array}{l}154 \\
10 ?\end{array}$ & & & 1 & & & & & \\
\hline 165 & $.70 n 11 n+n 3$ & $.53333+02$ & inh & $\begin{array}{c}-3500 n+c 3 \\
0\end{array}$ & .3 & it & & & & & \\
\hline 160 & $.70 n 0 n+n 3$ & . ${ }^{n}(\ln 00 n+02$ & 170 & $.2^{2} 8000$ & onor & 171 & & & ! & & \\
\hline 173 & . PAnOnan 3 &.$A c$ & 174 & . $280 \cap 0+0 ?$ & . $800 n 0+0 ?$ & 175 & 30000 & noo & & & \\
\hline 1 & $={ }^{2} 000 n+n s$ & . Tun & 178 & $.30000+03$ & $.60000+0 ?$ & 179 & .30000 & Ron & 180 & & \\
\hline & $-3 \ln 10 n+03$ & . has & 14? & & & 183 & 3 & 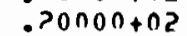 & 184 & -38 & \\
\hline 1 & 14 & & 1 Ah & & & 7 & & & 5 & & .533 \\
\hline & $n .0 n+n 3$ & $n+n 2$ & 190 & $.370 n(0)+03$ & $h 1+10 ?$ & 191 & $n 0 n+n 3$ & $333+02$ & $19 ?$ & $.32000+03$ & $80000+02$ \\
\hline
\end{tabular}




\begin{tabular}{|c|c|c|c|c|c|c|c|c|c|c|c|c|}
\hline$\because$ & b6 & $3 A$ & 35 & 63 & 54 & $4 A$ & 37 & 36 & 47 & 53 & 64 & 65 \\
\hline 9 & n9 & $a_{1}$ & 38 & 66 & 55 & 19 & no & 39 & 48 & 54 & 67 & 68 \\
\hline 10 & 72 & 144 & $a_{1}$ & 69 & 56 & 50 & 43 & 42 & 89 & 55 & 70 & 71 \\
\hline 11 & $\mathrm{BH}$ & no & 57 & 85 & 80 & 74 & 59 & 58 & 73 & 79 & 86 & 77 \\
\hline 12 & 71 & ${ }^{63}$ & to & RA & 81 & 75 & $A_{2}$ & $h_{1}$ & 74 & Ro & $\begin{array}{l}89 \\
02\end{array}$ & 80 \\
\hline 13 & $\begin{array}{l}24 \\
0.7\end{array}$ & no & t3 & 91 & $\begin{array}{l}a 2 \\
a z\end{array}$ & 76 & 65 & 64 & 75 & $\begin{array}{l}R_{1} \\
8_{2}\end{array}$ & $\begin{array}{l}02 \\
02\end{array}$ & 03 \\
\hline $\begin{array}{l}14 \\
115\end{array}$ & $\begin{array}{r}97 \\
100\end{array}$ & $\begin{array}{l}\text { 64 } \\
72\end{array}$ & 66 & $\begin{array}{l}9.4 \\
097\end{array}$ & $\begin{array}{l}A_{3} \\
8_{4}\end{array}$ & $\begin{array}{l}77 \\
78\end{array}$ & $\begin{array}{r}48 \\
77\end{array}$ & $\begin{array}{l}67 \\
70\end{array}$ & 76 & $\begin{array}{l}82 \\
83\end{array}$ & $\begin{array}{l}95 \\
98\end{array}$ & $\begin{array}{l}96 \\
09\end{array}$ \\
\hline ih & 114 & $8 \mathrm{P}$ & P\&s & 111 & 107 & جח & $\begin{array}{l}11 \\
87\end{array}$ & $A_{B}$ & 101 & $1 n_{n}$ & i1 & $\begin{array}{r}99 \\
113\end{array}$ \\
\hline 17 & 117 & 91 & คค & 114 & 108 & 103 & 90 & RY & 102 & 107 & 115 & 116 \\
\hline 18 & $1>0$ & 014 & 91 & 117 & $\ln _{4}$ & $\ln _{4}$ & 03 & $9 ?$ & 103 & $1 \cap A$ & $11 \mathrm{H}$ & 119 \\
\hline $1^{n}$ & $1>3$ & 97 & 94 & $1>0$ & 110 & 105 & 96 & 95 & 104 & 109 & $1>1$ & $1 ? 2$ \\
\hline$? 0$ & 137 & 114 & 111 & 134 & 131 & 175 & 113 & 112 & $1>4$ & $1>9$ & 135 & 136 \\
\hline 21 & $1 / 10$ & 111 & 114 & 137 & $1 \times 1$ & $1>h$ & 116 & 115 & $1>5$ & 130 & 138 & 139 \\
\hline ? & $1^{1 / 3}$ & $1>0$ & 117 & 140 & 132 & $1>7$ & 119 & 118 & $1 ? 6$ & 131 & 111 & 1112 \\
\hline$? 3$ & 140 & 173 & $1>0$ & 103 & 133 & 178 & 172 & $1 \geq 1$ & $1>7$ & 132 & 144 & 145 \\
\hline 24 & 160 & 137 & 134 & 157 & 143 & 144 & 136 & 135 & 147 & 152 & 158 & 159 \\
\hline 25 & 163 & 1110 & 137 & 160 & 154 & 149 & 139 & 138 & $14 \mathrm{R}$ & 153 & 151 & 142 \\
\hline ?h & 1 ho & 1113 & 140 & 16.3 & 155 & 150 & 172 & $1 / 1$ & 149 & 154 & 164 & 165 \\
\hline 27 & 149 & 146 & 1113 & 166 & $15 \mathrm{~A}$ & 151 & 14 & 11 & 150 & 155 & 167 & 16 \\
\hline 2R & 143 & $16 ?$ & 157 & 180 & 176 & 171 & 150 & 15 & 17 & 175 & 1A1 & 192 \\
\hline ?n & $18 \mathrm{~h}$ & 163 & (h) & 183 & 177 & 178 & Ih & 16 & 171 & 170 & $1^{24}$ & 185 \\
\hline 30 & בחו & $\begin{array}{l}1 \text { the } \\
\end{array}$ & 163 & $\begin{array}{l}18 \mathrm{~h} \\
189\end{array}$ & $\begin{array}{l}178 \\
179\end{array}$ & 173 & las & 164 & $\begin{array}{r}172 \\
173\end{array}$ & $\begin{array}{l}177 \\
178\end{array}$ & $\begin{array}{l}187 \\
100\end{array}$ & $1.8 \mathrm{~B}$ \\
\hline 31 & $\ln _{2}$ & 169 & lat & 189 & 179 & 174 & 148 & 167 & 173 & 178 & 100 & 191 \\
\hline
\end{tabular}




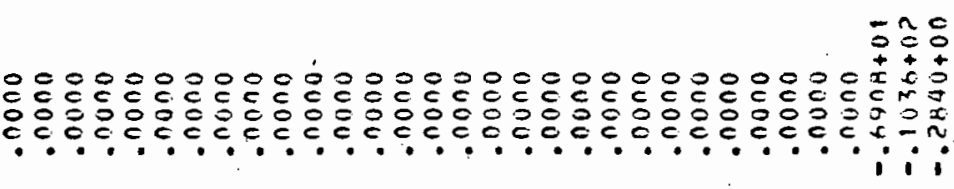

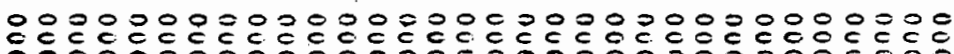

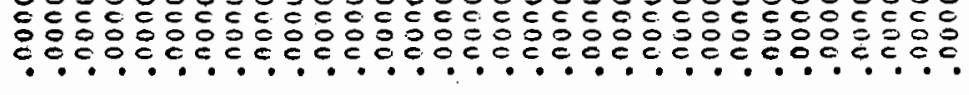

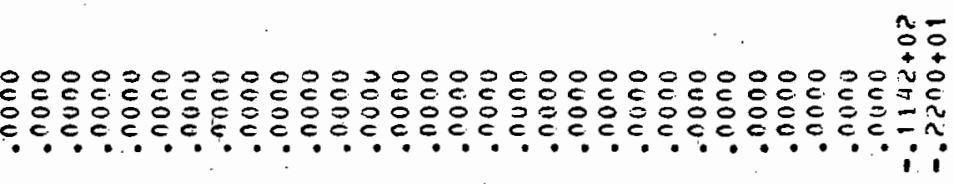

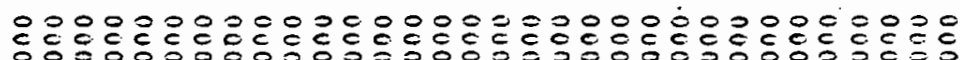

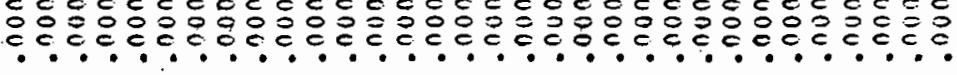

o

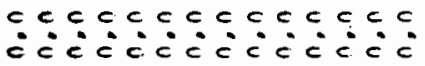

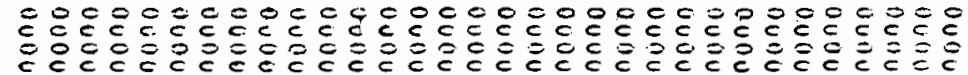

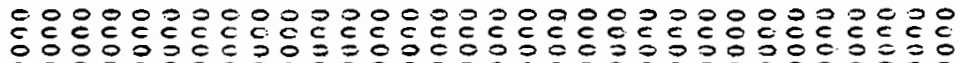
$\because \overrightarrow{0}$

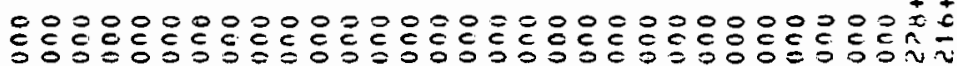

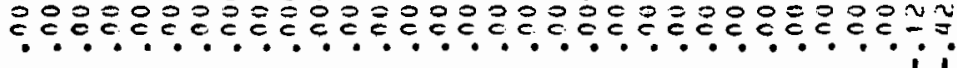
.

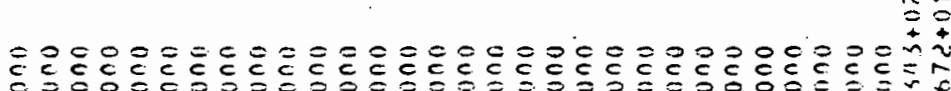

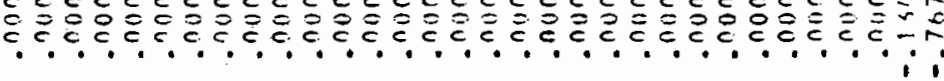

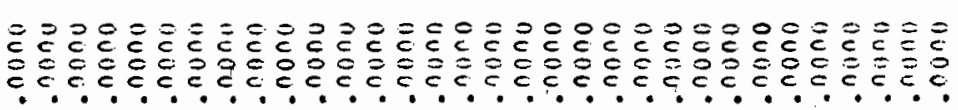

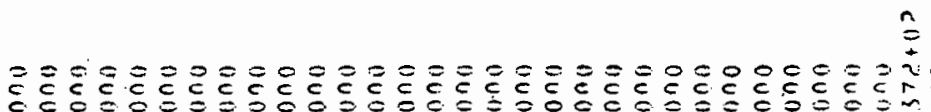

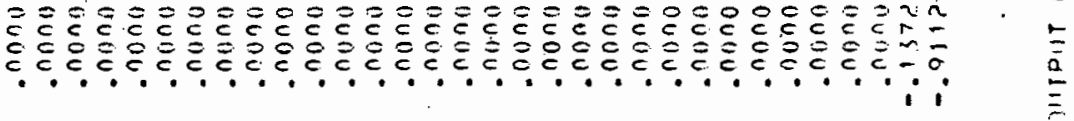


$\ddot{\sigma}$

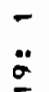

$\ddot{x}$

$\ddot{-}$

兽

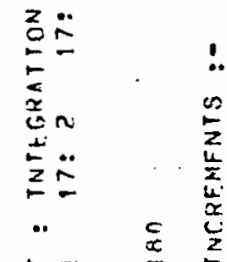

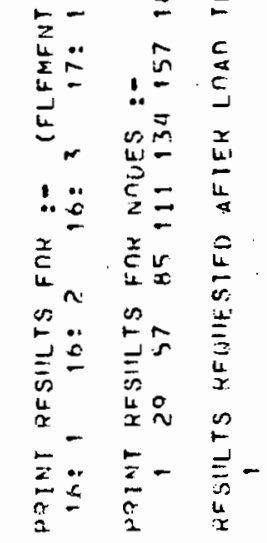


AT LONO FACTOR. . 169903+01

LMAN VECIMR 1 : CUMULATIVE LOAN FACIMR $=.169503+01 ; \quad\{$ Total $=1.695 \times 110.59=187\}$

\begin{tabular}{|c|c|c|c|c|c|c|c|c|c|c|c|c|}
\hline $\begin{array}{l}\text { FIFME } \\
\text { IVI. }\end{array}$ & $\begin{array}{l}\text { nI, } \\
\text { OT. }\end{array}$ & $\begin{array}{l}\text { GIOAAL } \\
\text { CnOROS }\end{array}$ & $\begin{array}{l}\text { STOAIN } \\
\text { ELASTIC }\end{array}$ & $\frac{\text { ICREMENTS }}{\text { PLASTIC }}$ & LAMADA & $\begin{array}{l}\text { SIRESS } \\
\text { INCREMENT }\end{array}$ & ELASTIC & $\begin{array}{l}\text { STRATNS } \\
\text { PLASTIC }\end{array}$ & TUTAL & STRFSS & $\begin{array}{l}\text { YTELD } \\
\text { FUNCTION }\end{array}$ & $\begin{array}{l}\text { STRESS } \\
\text { CORRECTION } \\
\text { FACTOP }\end{array}$ \\
\hline 16 & $\begin{array}{r}x \\
y \\
x y\end{array}$ & $\begin{array}{l}-\log _{0} n+n 3 \\
.>454 n+n 1\end{array}$ & $\begin{array}{r}-41871-03 \\
.11304-03 \\
-.16708-07\end{array}$ & $\begin{array}{l}\cdots \\
\cdots\end{array}$ & -- & $\begin{array}{r}.41871+00 \\
-11399+00 \\
-.83542+00\end{array}$ & $\begin{array}{r}11871-03 \\
-11305-03 \\
-.1670 H-0 ? \text { ? }\end{array}$ & $\begin{array}{l}.00000 \\
\text { nonon } \\
\text { nonon }\end{array}$ & $\begin{array}{r}.41871-03 \\
.11398-03 \\
-.16708-0 ?\end{array}$ & $\begin{array}{r}.91871+n 0 \\
.11399+n 0 \\
-.8354 ?+10\end{array}$ & $-.10218+04$ & -- \\
\hline 16 & $\begin{array}{l}y \\
y \\
X Y\end{array}$ & $\begin{array}{l}-1 \ln 0 u n+n z \\
.10 \times 47+n z\end{array}$ & $\begin{array}{r}.10766-07 \\
.60577-02 \\
-.12330-07\end{array}$ & $=-$ & -- & $\begin{array}{r}10766+01 \\
-64527+00 \\
-.81650+00\end{array}$ & $\begin{array}{r}.1074 h-0 ? \\
.695>7-03 \\
-.14330-0 ?\end{array}$ & $\begin{array}{l}.00000 \\
.00000 \\
.00000 .\end{array}$ & $\begin{array}{r}.10766-0 ? \\
.60577-03 \\
-.16330-02\end{array}$ & $\begin{array}{r}10706+01 \\
.64527+00 \\
-.81650+00\end{array}$ & $-.10211+0 n$ & $\cdots$ \\
\hline $\begin{array}{r}16 \\
3\end{array}$ & $\begin{array}{r}y \\
y \\
X Y\end{array}$ & $\begin{array}{l}-16 n 0 n+n 3 \\
.19321+n ?\end{array}$ & $\begin{array}{r}-17393-07 \\
-17859-07 \\
-.18953-0 ?\end{array}$ & $\because-$ & -- & $\begin{array}{r}.17393+01 \\
.12859+01 \\
-.94701+00\end{array}$ & $\begin{array}{r}.17393-0 ? \\
-17459-0 ? \\
-.16953-02\end{array}$ & $\begin{array}{l}\text { nonon } \\
\text { nonon } \\
\text { nonon }\end{array}$ & $\begin{array}{r}11393-0 ? \\
.17859-0 ? \\
-.16953-0 ?\end{array}$ & $\begin{array}{r}17393+01 \\
-12859+01 \\
-84764+00\end{array}$ & $-.10194+04$ & - \\
\hline 17 & $\begin{array}{r}x \\
y \\
X Y\end{array}$ & $\begin{array}{l}.16000+0.3 \\
.74220+12\end{array}$ & $\begin{array}{r}-224 \cap 3-0 ? \\
-149 ? 4-0 ? \\
-.17531-4) ?\end{array}$ & $=$ & -- & $\begin{array}{r}.22903+01 \\
.14929+01 \\
-.97655+00\end{array}$ & $\begin{array}{r}.22403-02 \\
-11928-02 \\
-.17521-0 ?\end{array}$ & $\begin{array}{l}.00 n 0 n \\
.00 n 00 \\
.00000\end{array}$ & $\begin{array}{r}.22803-02 \\
-14928-07 \\
-.17531-02\end{array}$ & $\begin{array}{r}-22 A 03+01 \\
-14928+01 \\
-.87655+00\end{array}$ & $-.10177+04$ & $\cdots$ \\
\hline $\begin{array}{r}17 \\
?\end{array}$ & $\begin{array}{r}x \\
y \\
x y\end{array}$ & $\begin{array}{l}-10 n 0 n+03 \\
.3 p 662+02\end{array}$ & $\begin{array}{r}.34 \sin 1-0 ? \\
.21 \sin 9-0 ? \\
-.2 \sin 4-i) ?\end{array}$ & $=$ & $=-$ & $\begin{array}{r}34601+01 \\
.71909+01 \\
-.10797+01\end{array}$ & $\begin{array}{r}.31001-0 ? \\
.21809-0 ? \\
-.20594-0 ?\end{array}$ & $\begin{array}{l}\text { nonon } \\
\text { nonoo } \\
\text { nonon }\end{array}$ & $\begin{array}{r}.34691-0 ? \\
.21809-0 ? \\
-.20594-0 ?\end{array}$ & $\begin{array}{r}.34601+n 1 \\
.21809+n 1 \\
-.10297+n 1\end{array}$ & $-.10116+04$ & -- \\
\hline $\begin{array}{r}17 \\
3\end{array}$ & $\begin{array}{r}Y \\
Y \\
X Y\end{array}$ & $\begin{array}{l}-18000+03 \\
.41095+0 \%\end{array}$ & $\begin{array}{r}.47274-07 \\
.20270-07 \\
-.25374-07\end{array}$ & $=$ & -- & $\begin{array}{r}.17>79+n 1 \\
.79720+n 1 \\
-.1310^{n+n+n 1}\end{array}$ & $\begin{array}{r}47279-02 \\
.29270-02 \\
-.24378-0 ?\end{array}$ & $\begin{array}{l}.00000 \\
.00000 \\
.00000\end{array}$ & $\begin{array}{r}.47279-07 \\
.29270-0 ? \\
-.26378-0 ?\end{array}$ & $\begin{array}{r}.47279+01 \\
.29720+01 \\
-.15180+01\end{array}$ & $-.10017+04$ & $-\infty$ \\
\hline 19 & $\begin{array}{r}y \\
y \\
X Y\end{array}$ & $\begin{array}{l}.1 \ln n 0 n+n 3 \\
.4580 x+n ?\end{array}$ & $\begin{array}{r}.515>3-07 \\
.34947-0 ? \\
-.2145>5-02\end{array}$ & $\begin{array}{l}-- \\
--\end{array}$ & -- & $\begin{array}{r}-51923+01 \\
.36047+01 \\
-.12203+01\end{array}$ & $\begin{array}{r}.51523-01 ? \\
.36947-11 ? \\
-.2 .1525-02\end{array}$ & $\begin{array}{l}\text { nonon } \\
\text { nonon } \\
\text { non ono0 }\end{array}$ & $\begin{array}{r}.515 ? 3-0 ? \\
.36947-0 ? \\
-.245 ? 3-0 ?\end{array}$ & $\begin{array}{r}.51523+01 \\
.36047+01 \\
-.12263+01\end{array}$ & $-.99833+03$ & $\cdots$ \\
\hline$!^{R}$ & $\begin{array}{r}y \\
y \\
x y\end{array}$ & $\begin{array}{l}-10000+103 \\
-53540+12\end{array}$ & $\begin{array}{r}.53950-0 ? \\
.44505-11 ? \\
-.18475=0 ?\end{array}$ & $=$ & - & $\begin{array}{r}53050+01 \\
.44309+01 \\
-.04624+00\end{array}$ & $\begin{array}{r}.53950-0 ? \\
.41305-10 ? \\
-.18975-0 ?\end{array}$ & $\begin{array}{l}n=0000 \\
.00000 \\
.00000\end{array}$ & $\begin{array}{r}.53950-0 ? \\
.41305-0 ? \\
-.19975-0 ?\end{array}$ & $\begin{array}{r}53950+01 \\
.44305+01 \\
-.04624+00\end{array}$ & $-.99648+03$ & $\cdots$ \\
\hline $\begin{array}{r}19 \\
3\end{array}$ & $x_{x y}^{x}$ & $\begin{array}{l}-16000+03 \\
.61>95+02\end{array}$ & $\begin{array}{r}.47002-112 \\
.57204-02 \\
-.21331-102\end{array}$ & $\begin{array}{l}\because- \\
--\end{array}$ & -- & $\begin{array}{r}-42002+n 1 \\
-52744+n 1 \\
-\therefore 10666+11\end{array}$ & $\begin{array}{r}.0 P 00 ?-0 ? \\
.5 P 201-0 ? \\
-.21331-0 ?\end{array}$ & $\begin{array}{l}.00000 \\
.00000 \\
00000\end{array}$ & $\begin{array}{r}.070 \cap 2-0 ? \\
.37294-0 ? \\
-.21331-0 ?\end{array}$ & $\begin{array}{r}-62007+n 1 \\
-5 ? 744+n 1 \\
-.10606+n 1\end{array}$ & $-.9 A 7>2+03$ & - \\
\hline 19 & $\begin{array}{r}x \\
y \\
x y\end{array}$ & $\begin{array}{l}.16025+03 \\
.45402+02\end{array}$ & $\begin{array}{r}.44958-0 ? \\
.71563-0 ? \\
-.19794=0 ?\end{array}$ & $\begin{array}{l}-- \\
--\end{array}$ & -- & $\begin{array}{r}.84958+01 \\
.71563+n 1 \\
-.03027+n 0\end{array}$ & $\begin{array}{r}.84954-0 ? \\
.71563-0 ? \\
-.197844-0 ?\end{array}$ & $\begin{array}{l}\text { nonoon } \\
\text { nonon } \\
\text { nonon }\end{array}$ & $\begin{array}{r}.84998-0 ? \\
.71543-0 ? \\
-.: 9784-0 ?\end{array}$ & $\begin{array}{r}.8495 A+01 \\
.71563+01 \\
-.93027+00\end{array}$ & $-.95876+03$ & -- \\
\hline $\begin{array}{r}10 \\
?\end{array}$ & $\begin{array}{r}x \\
y \\
X Y\end{array}$ & $\begin{array}{l}-16113+n 3 \\
.71775+n z\end{array}$ & $\begin{array}{r}-114443-01 \\
-44210-02 \\
-.11734-01\end{array}$ & $\begin{array}{l}-- \\
--\end{array}$ & -- & $\begin{array}{r}14 a_{6} 3+0 ? \\
-24210+01 \\
-.58491+01\end{array}$ & $\begin{array}{r}-11443-01 \\
-81210-0 ? \\
-.1173 A-01\end{array}$ & $\begin{array}{l}.00000 \\
.00000 \\
.00000\end{array}$ & $\begin{array}{r}14963-01 \\
.84210-0 ? \\
-.11734-01\end{array}$ & $\begin{array}{r}111963+02 \\
-84710+01 \\
-.58691+01\end{array}$ & $-.75187+03$ & $\cdots$ \\
\hline 19 & $x$ & $.16>0 n+n 3$ & $.2 \times 39 b-01$ & .00000 & & .36345402 & $.26305-01$ & .00000 & $.26305-01$ & $.26395+02$ & & \\
\hline
\end{tabular}




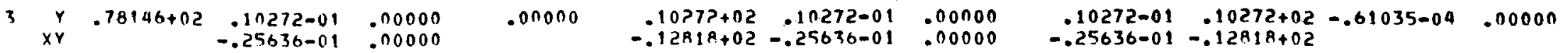

CIIRRENT PLASTIC POINTS - (ELEMENT : INTFGRATION POINT)

19: 3

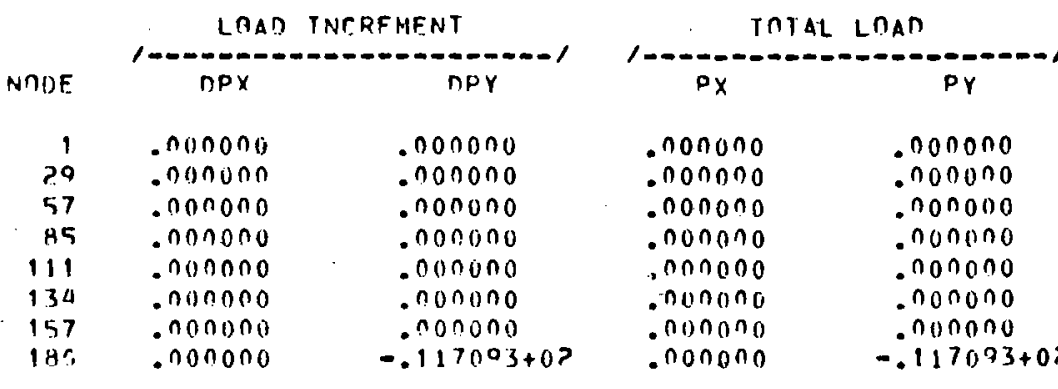

DISPLACFMENT INCREMENT

$$
\text { nU }
$$

.000000

$.00 n 0 n$

inonona

inonona

. Donana

coununa

. 0000000

ov

.000000 $-1917631-0$ $-.18308 B+0.0$ $=.193479+00$ $\because 141792+01$ -
$-.263791+01$ $-.263791+01$
$-.442992+01$
TOTAL DISPLACFMENT

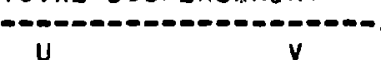

000000 .000000 .000000
$\therefore .194631-01$

- nonono $\quad-.493479+00$

- Donono $\quad-$.

. nonono $\quad-.141702+01$

$.000000 \quad \because 442982+01$ 
END OF LOADTNG 1 : MAXIMUM LTAD RFACHED AT NODE IRO

AT LUAD FACTOR . $238755-01$

LMAO VETIMK 1 : CIMMILATIVE LOAD FACTOH = .53PQAG+0I
$\Rightarrow P=5.32986 \times 110.59=589.429$

CURRENT VALUES

\begin{tabular}{|c|c|c|c|c|c|c|c|c|c|c|c|c|}
\hline \multirow{2}{*}{\multicolumn{2}{|c|}{$\begin{array}{l}\text { FI.FMENT. } \\
\text { INT. PT. }\end{array}$}} & \multicolumn{3}{|c|}{ STPATN INCREMENIS } & \multirow[b]{2}{*}{ (AMAB) } & \multirow{2}{*}{\multicolumn{6}{|c|}{ STRESS }} & \multirow[b]{2}{*}{$\begin{array}{l}\text { STRESS } \\
\text { CORRECTION } \\
\text { FACTOR. }\end{array}$} \\
\hline & & $\begin{array}{l}\text { G! } 00 \text { Al } \\
\operatorname{cog} 005\end{array}$ & ELASTIC & $P_{L A S T I C}$ & & $\begin{array}{l}\text { STRESS } \\
\text { INCPEMENT }\end{array}$ & & PLastic & TOTAL & STRESS & $\begin{array}{l}\text { YIELD } \\
\text { FUNCTION }\end{array}$ & \\
\hline $\begin{array}{c}1 \mathrm{~h} \\
1\end{array}$ & $\begin{array}{r}x \\
y \\
X Y\end{array}$ & $\begin{array}{l}.16 n 00+n 3 \\
.21354 n+n 1\end{array}$ & $\begin{array}{r}.2 n 462-04 \\
.8758 ?-05 \\
-.16041-03\end{array}$ & $\begin{array}{l}- \\
\because- \\
-\end{array}$ & -- & $\begin{array}{r}. P 01862-01 \\
-A 758 ?-02 \\
-.90>07-01\end{array}$ & $\begin{array}{r}.21920-02 \\
.31892-03 \\
-.11149-01\end{array}$ & $\begin{array}{l}\text { nonon } \\
\text { nonon } \\
\text { nonon }\end{array}$ & $\begin{array}{r}.21930-02 \\
-31892-03 \\
-.11169-01\end{array}$ & $\begin{array}{r}. ? 1930+01 \\
.3189 ?+00 \\
-.55847+01\end{array}$ & $-.92622+03$ & $\therefore$ \\
\hline in & $\begin{array}{r}x \\
y \\
X Y\end{array}$ & $\begin{array}{l}\operatorname{lon} \sin \ln 3 \\
\log 8 \log 2\end{array}$ & $\begin{array}{r}.32409-04 \\
.57823-04 \\
-.143>7-113\end{array}$ & $\because$ & -- & $\begin{array}{r}.321109-01 \\
.57823-01 \\
-.71633-01\end{array}$ & $\begin{array}{r}.53445-02 \\
.39193-0 ? \\
-.113 \cap 2-01\end{array}$ & $\begin{array}{l}.00 n 0 n \\
\text { ononon } \\
\text { - oonon }\end{array}$ & $\begin{array}{r}.53445-0 ? \\
.39193-0 ? \\
-.113 \cap 2-01\end{array}$ & $\begin{array}{r}.53445+n 1 \\
.38193+n 1 \\
-.56512+01\end{array}$ & $-.90545+03$ & - \\
\hline 19 & $\begin{array}{r}y \\
y \\
X Y\end{array}$ & $\begin{array}{l}-16000+n 3 \\
-19321+n 2\end{array}$ & $\begin{array}{r}.49691-n 11 \\
-10374-03 \\
-.14290-03\end{array}$ & $\begin{array}{l}-- \\
--\end{array}$ & -- & $\begin{array}{r}.49 h A 1-01 \\
.10874+00 \\
-.71449-n 1\end{array}$ & $\begin{array}{r}.86 / 48-1) 2 \\
.79779-02 \\
-124112-01\end{array}$ & $\begin{array}{l}.00 n 00 \\
.00000 \\
.00000\end{array}$ & $\begin{array}{r}.46748-02 \\
.719779-02 \\
-.12442-01\end{array}$ & $\begin{array}{r}.80749+n 1 \\
.74779+01 \\
-.62710+01\end{array}$ & $-.84160+03$ & -- \\
\hline $\begin{array}{r}17 \\
1\end{array}$ & $\begin{array}{l}X \\
y \\
X Y\end{array}$ & $\begin{array}{l}.1600 n+n 3 \\
.74720+02\end{array}$ & $\begin{array}{r}.12654-03 \\
-11872-113 \\
-.421>1-104\end{array}$ & $=$ & $-\infty$ & $\begin{array}{r}136511+00 \\
-11872+00 \\
-.16561-n 1\end{array}$ & $\begin{array}{r}.11567-01 \\
.89263-1) ? \\
-.11779-01\end{array}$ & $\begin{array}{l}.00000 \\
.00000 \\
.00000\end{array}$ & $\begin{array}{r}.11 \\
.89 \\
-.11\end{array}$ & $\begin{array}{r}-11567+02 \\
-89263+01 \\
-.54645+01\end{array}$ & -.81060703 & -- \\
\hline $\begin{array}{r}17 \\
?\end{array}$ & $\begin{array}{r}x \\
y \\
X Y\end{array}$ & $\begin{array}{l}-\ln \ln n n+n 3 \\
-32 \operatorname{sh} 2+n 2\end{array}$ & $\begin{array}{r}.39440-07 \\
.15335-03 \\
-.211740-03\end{array}$ & $\begin{array}{l}-- \\
--\end{array}$ & - & $\begin{array}{r}-39460+n 0 \\
-15335+n 0 \\
-.1237 n+00\end{array}$ & $\begin{array}{r}17775-01 \\
.13071-01 \\
-.13454-01\end{array}$ & $\begin{array}{l}.00000 \\
.00 n 00 \\
.00000\end{array}$ & $\begin{array}{r}.17775-01 \\
.13071-01 \\
-.13854-01\end{array}$ & $\begin{array}{r}17775+02 \\
.13021+02 \\
-.69 ? 7 ?+01\end{array}$ & $-.626 n 0+03$ & -- \\
\hline $\begin{array}{r}17 \\
3\end{array}$ & $\begin{array}{r}X \\
Y \\
X Y\end{array}$ & $\begin{array}{l}-\ln 000 n+n 3 \\
-\ln \ln 95+n 2\end{array}$ & $\begin{array}{r}.71277-(1) 3 \\
.19784-(1) \\
-.47579-03\end{array}$ & $\begin{array}{l}-- \\
--\end{array}$ & -- & $\begin{array}{r}.71727+00 \\
-19784+100 \\
-.73790+100\end{array}$ & $\begin{array}{r}.25674-01 \\
.17619-01 \\
-.18647-01\end{array}$ & $\begin{array}{l}.00 n 00 \\
\text { nonoo } \\
\text {. nonon }\end{array}$ & $\begin{array}{r}.25679-01 \\
.17619-01 \\
-.18647-01\end{array}$ & $\begin{array}{r}.25679+02 \\
.17619+102 \\
-.93734+01\end{array}$ & $-.24581+03$ & -- \\
\hline $\begin{array}{r}1 \mathrm{~A} \\
1\end{array}$ & $\begin{array}{r}X \\
Y \\
X Y\end{array}$ & $\begin{array}{l}-16 n 0 n+n z \\
.45=03+n 2\end{array}$ & $\begin{array}{l}.11007-013 \\
.10953-04 \\
.23133-03\end{array}$ & $\begin{array}{r}.91771-03 \\
.25685-03 \\
-.06025-13\end{array}$ & $.13475-102$ & $\begin{array}{l}.11607+00 \\
.1995 z-01 \\
.11567+00\end{array}$ & $\begin{array}{r}.33071-01 \\
.27594-01 \\
. .19965-01\end{array}$ & $\begin{array}{r}.02495-02 \\
.12421-02 \\
-.47683-02\end{array}$ & $\begin{array}{r}.37320-01 \\
-23837-01 \\
-.19734-01\end{array}$ & $\begin{array}{r}.33 n 5 h+n 2 \\
.22584+n 2 \\
-.74792+n 1\end{array}$ & $-.15259-04$ & $.99997+00$ \\
\hline $\begin{array}{r}18 \\
?\end{array}$ & $\begin{array}{r}X \\
Y \\
X Y\end{array}$ & $\begin{array}{l}-16 n 0 n+n 3 \\
.53549+02\end{array}$ & $\begin{array}{r}.70 a n 3-0 a \\
-.331 h H-05 \\
.17062-03\end{array}$ & $\begin{array}{r}.17169-02 \\
.24814-03 \\
-.15866-02\end{array}$ & $53-02$ & $\begin{array}{r}79103-01 \\
-3316 R-02 \\
.85311-01\end{array}$ & $\begin{array}{r}.33935-01 \\
.29858-01 \\
-.11274-01\end{array}$ & $\begin{array}{r}.33561-01 \\
.64846-02 \\
-.00054-01\end{array}$ & $\begin{array}{r}.07496-01 \\
.27342-01 \\
-.54378-01\end{array}$ & $\begin{array}{r}.33919+02 \\
.20782+02 \\
-.71055+01\end{array}$ & $-.3 n 518-04$ & $.99999+100$ \\
\hline $\begin{array}{r}18 \\
3\end{array}$ & $\begin{array}{r}X \\
Y \\
X Y\end{array}$ & $\begin{array}{l}-16000+n 3 \\
.61 ? 95+n 2\end{array}$ & $\begin{array}{r}.51300-04 \\
-.15720-04 \\
.12121-03\end{array}$ & $\begin{array}{r}.>3053-n 2 \\
-P 1378-n 3 \\
-.>1412-n 2\end{array}$ & $.33714-02$ & $\begin{array}{r}.51300-01 \\
-.15736-01 \\
.60403-01\end{array}$ & $\begin{array}{r}.34624-01 \\
.19378-01 \\
-.13609-01\end{array}$ & $\begin{array}{r}.71654-01 \\
.96121-02 \\
-.91751-01\end{array}$ & $\begin{array}{r}.10628+00 \\
.28940-01 \\
-.95360-01\end{array}$ & $\begin{array}{r}.34334+02 \\
.19160+02 \\
. .67306+01\end{array}$ & $-.30518-04$ & $.99999+00$ \\
\hline 10 & $\begin{array}{l}x \\
y \\
x y\end{array}$ & $\begin{array}{l}-10025+n 3 \\
-\operatorname{son} 103+n ? 0\end{array}$ & $\begin{array}{r}.37225-044 \\
-.472,25-018 \\
.57048-04\end{array}$ & $\begin{array}{r}.34>02-n 2 \\
-11504-13 \\
-.3253 h-n ?\end{array}$ & $.47453-07$ & $\begin{array}{r}-22335-n 1 \\
-\because 12535-n 1 \\
.74544-n 1\end{array}$ & $\begin{array}{r}.33814-01 \\
.10716-01 \\
-.15071-01\end{array}$ & $\begin{array}{r}12527+00 \\
.16555-01 \\
-.13067+00\end{array}$ & $\begin{array}{r}.15908+0 n \\
.36312-01 \\
-.14569+00\end{array}$ & $\begin{array}{r}r 3625+n 2 \\
.19629+n 2 \\
-.74 A 4 R+n 1\end{array}$ & $-.45776-04$ & $.10000+n 1$ \\
\hline $\begin{array}{l}19 \\
?\end{array}$ & $\begin{array}{l}x \\
y \\
x y\end{array}$ & $\begin{array}{l}.16113+n 3 \\
.71775+n 2\end{array}$ & $\begin{array}{l}-.30543-05 \\
\because .0160-01 \\
-.55604-115\end{array}$ & $\begin{array}{r}.54645-n ? \\
.3453 ?-n 3 \\
-.11894 n-n ?\end{array}$ & $.76556-0 ?$ & $\begin{array}{l}=.10543-n 2 \\
=.1916 n-n 1 \\
=.7780>-n ?\end{array}$ & $\begin{array}{r}.35094-01 \\
.192197-01 \\
-.14338-01\end{array}$ & $\begin{array}{r}.27602+00 \\
-21645-01 \\
-.26824+00\end{array}$ & $\begin{array}{r}.31112+00 \\
.40912-01 \\
-.28254+00\end{array}$ & $\begin{array}{r}.34213+02 \\
-18719+02 \\
-.69164+01\end{array}$ & -.45 & $.10000+01$ \\
\hline
\end{tabular}


$10 \times \quad .16200+03-.31533-05 \quad .94248-02 \quad-.31533-02 \quad .36005-01 \quad .05058+00 \quad .48658+00 \quad .34573+02$

$y \quad .7814 A+n 2-.21338-04 \quad .12314-03 \quad .10630-01-.2133 A-01 \quad .193 A 1-01 \quad .28640-01 \quad .48031-01 \cdot .16537+02-.3051 B-04 \quad .10000+01$ $x y \quad-.11030-04-.64681-n 2$

$-55149-02-.13844-01-.111195+00-.42579+00=.64794+01$

CIHRENT PIASTIC POINIS - TEIEMFNT: [MTFGRATION POINI]
13: 7
13:8 13:?
14: 7
14: A $1 \mathrm{H}: 1$
18: ? :19:3
$18: 5$
18:6
$18: 9 \quad 19: 1$
19: 2
19:3 19:4

19:5 14:6 19:7 19:0 ?3:3

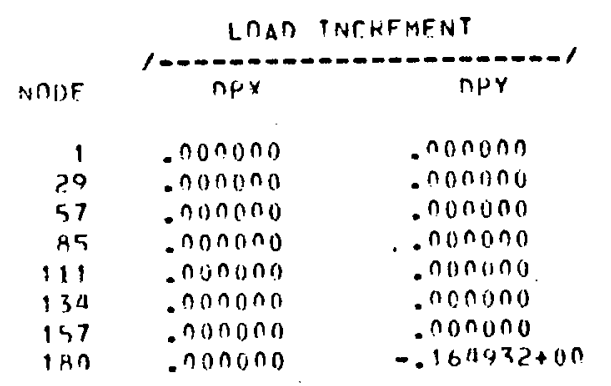

\begin{tabular}{ll} 
\\
\hline px
\end{tabular}

DISPLACFMFNT INCRFMENT

DU
OISPLACEMENT INCREMENT

TOTAL OISPLACFMFNT

$$
\text { nu }
$$

.000000

$.00 n 0 n 0$

- nonono

$.00 n \operatorname{lono}$
.00000

$-.740011-03$
$-.452086-02$

$-4528 R 6-02$
$-.186979-01$

$-186979-0$
$-366278-01$

$\because 653602-01$

oconso

- $11607 ?+00$

$-179059+00$

nunono

u

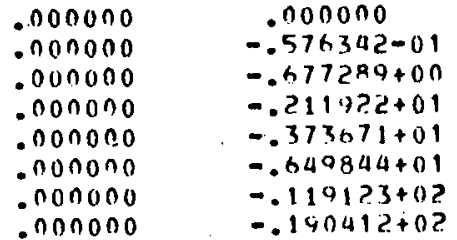


A.5 EPTCS Program Listing 
COL AEPTCS(1). MAIN

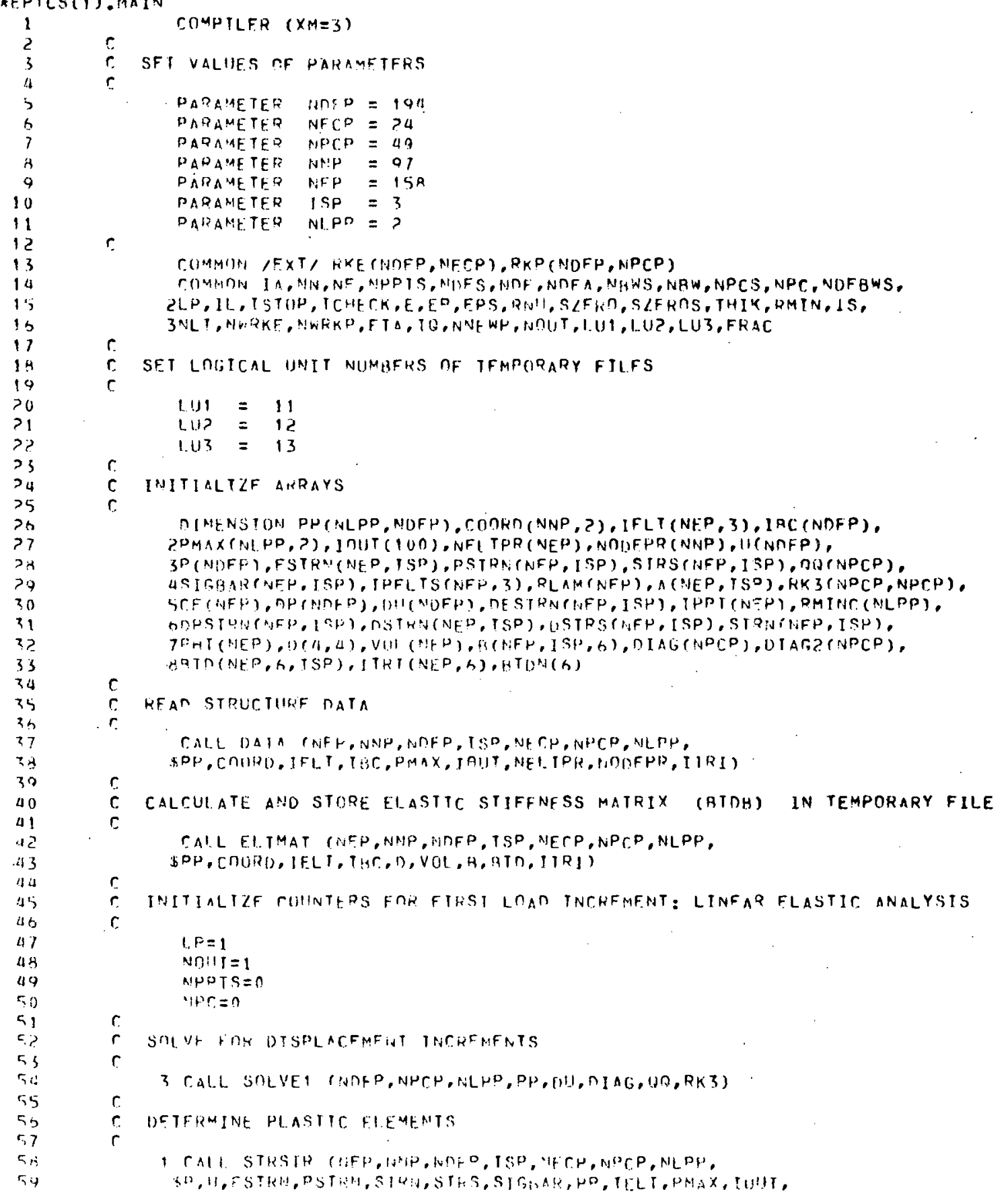


C EVALUATF DLASTIC MATRTCES

\section{$(-R I D N)$ AND (NTON+RP)}

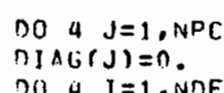

no $4 \mathrm{I}=1, \mathrm{~N} \cap \mathrm{F}$

RKP $(T, T)=0$.
NOS 5 IF $=1$, NE

NOS 5 IF $=1$, NE

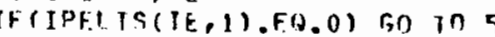

CALL PIASM (NEP, ISP, IE,A,SISBAR, VILL,D, BIDN,ENTDN, HTOS

NC, = TPELTS $(I E, Z)$

no $\quad f \quad l=1, n$

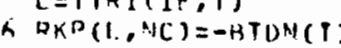

DIAG(iar) =ENIDR

5 TONTTHAR

ADPIY GOUNDARY CUNDITIONS TO PLASTIC Matrix (-ATDN)

nO $7 \quad 1=1$, RnF

IF(IDC(I). FO.1) ro in 7

ni) \& $J=1, N R$

Q $\operatorname{DKP}(1, J)=0$

SOLVE, RHFCK KINEMATIC CONSTRAINTS ON ELASTIC AND PLASTIC REGIONS

ANID ITEQATE IF NECESSARY

CALL ITER INEH, NNP, NOFP, ISP, NECP.NPCP, NLPP.

STELT, IAC, D, IPEI, TS, RLAM, A, PP, DU, SIGAAR, VOL, R, ITRI, KMINC

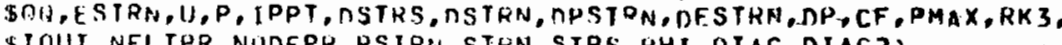

c

APPI Y NEXT LOAD INCPFMENT

rio in 1

$\int \sin \sin$

FND 
SURROUTINE DATA (NFP,NNP,NDFP, ISP, NECP, NPCP, NLPP,

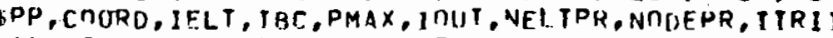

COMMON I $A, N N, N F, N P P T S, N A F S, N M F, N A F A, N A W S, N R W, N P C S, N P C, N D F B W S$,

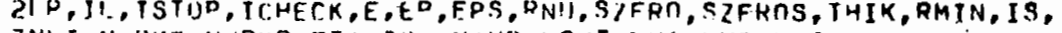

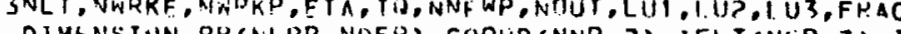

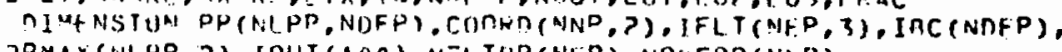

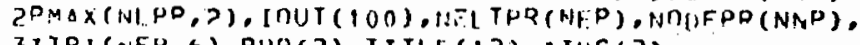

$r$

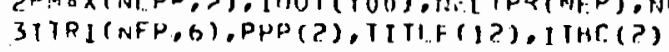

RFAD AND PHTHT HEADTHF,

PEAD 101,TITIE

O) FDPMAT(1)AG)

PRTNT ?OY, TITLE

PEAC) $101, T I T L F$

?01 FURMAT(1H1,1?Ah,1) $c$

ION FORNAT()

REAT TYPE OF ANALYSTS

QEAU 104,I,J

104 FODMAT(ZAG)

TF(J.E?.'STKFSS., TA $T A=-1$

TF(O).EO. 'STRAIN') TA=O

TF(J.E?. MFTAIT. $)$ TA

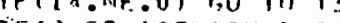

IF (J.FO. STHAIN') GO In 13

23 FODAAT(1HO, 3P('*'), /,' TYPF OF ANALYSIS INCORRECTLY SPECIFIED',

SIONALSTS TFRMINATEO', $1,39\left({ }^{\circ} *^{\circ}\right)$ )

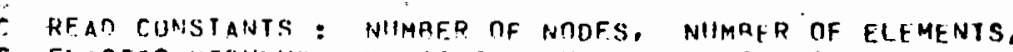

ELASTIC MOULLLIS, PLASITC MOUHLIIS, POISSON'S RATTO

STIAXTAL YIRLA SIDFSS. RATIO HF VON MISFS FQUIVAL.KN

AS DLASTIC. PLATF THTCKNFSS IF PLANE STHFSS ANALYSIS

13 IF(IA.FU.-1) RFAD IUO,NN,NF, F, FP, HNII,STERU,ETA, THIK, FRAC

IF(IA.GT,-1) RFAD 10O,NN,NF, F, F.P,RNU,STERO,ETA,FRAC

IF $(I A . F U .0)$ THTK $=1$.

$T F=$

FF(1A.F(1.- -1$) \quad 1 S=$

RFan Nonal cunRDinates

READ $100,((C O O R O P(, J), J=1,7), 1=1, N N)$

hFAR FLEMFNT INCINENCFS

DE $\triangle U$ IOD, (IFLT $(I, .),, J=1,3), I=1, N E)$

C. DETFKMINE CONSTAMTS FOK SOLUTION POIITINF. AND HALF BANO-WIDTH OF ELASTIC MATRIX

$A M W=0$

nu $1>1=1, \mathrm{NE}$

$I 1=\operatorname{Al} S(I F L T(I, 1)-I F i T(I, P))$ 
1?. IF (NRA,LT.MAX) NAW $M A X$

$N[S=(N A N+D) *$ ?

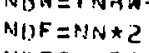

NISFS $=N M F-1$

NDF
NDF $=N D F+1$

NHWS
NHF

HDFHWS $=N D F-N A W S$

NARKF $=$ HUF $\star M M B W$

c

PRI"I HFAMINGS AND VALUES OF CONSTANIS

PKINT ?I?

1P FORMATI//, NUMERICAL ANALYSIS USING TRIANGULAR CONSTANT STRAIN FI 3NITE HLEMENTS', /1, PLANE STRESS, PLANE SIRAIN UR AXISYMMETRIT PRU BTSAMT, I

TF(IA.FW, -1) PRINI 215

SS ANALYSIS', I

TF(TA.FO.O) PRTNT ?Z?

2? FIRMAI(IHO, PLANF STRATN ANALYSIS, ,

IF (IA FB. 1) PEINT ?Q3

203 FIIRMAICIHO, 'AXTSYMMETHIC ANALYSIS', I/,' VARYING CIRCUMFERENIIAL SI SRATISS ANT SIRESSFS AVERAFT D AT CFNTKMIN OF ELEMENT SFCTION', DKTNT ?11

?I FUR IMAT(IHO.'VUN MISES YIFLO CANDITION?, I)

TF(t.D.HE.0.0) DUTHT ?I"

214. FIPMAT(1HO, 'KINEMATIC HAPNFNINGP, / IF (F.P.FH.0.0) PBITIT 221 , FRAC

221 FORMATCIHO.'FLASTIC, PFEFECITYY PLASTTC : ANALYSIS TFRMINATEO WHEN

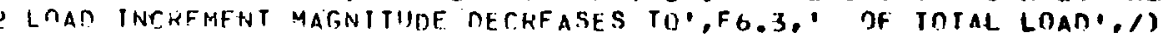
PRTUT ?47,FTA

P 7 FOMMAICHO. 'FLFMENTS WITH VON MISES FOUIVALENT STRFSS WITHIN', SFG.3.' nF YIELD STHFSS TRFATED AS PLASTIC ELEMENTS', PHTAT PSO, TIILE

220 FORYAI $(1 H 0,1$ TAK, $)$ $T=R \angle P P-1$

PKTNT $>13$, NNP, NEP, NDFP, NFCP, NPCP, I

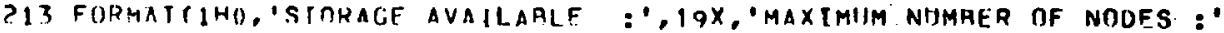

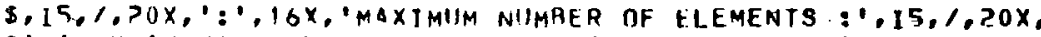

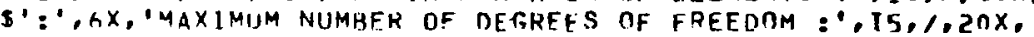

*': MAXTMIIM HALF RAND-WTDTH OF FLASTIC MATKTX : $, 15,1,20 X, 1,1,8 x_{0}$

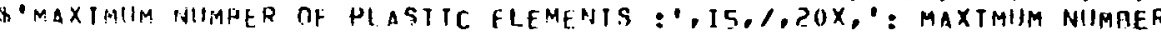

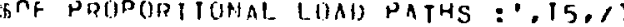

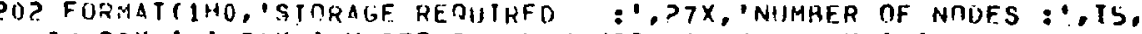

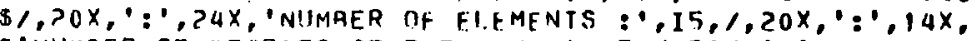
\$'NIIMRER TF DFl'GRES OF FRFENOM :', 15,1,20x,1:1,9x,

\$MALF BAND-WIDTH OF F.LASTIC MATKIX: $1,15,1)$

DHIVT PIT,F,FP, HNU, STED!

217 FU?NAT(1HO, MATERIAL CONSTANTS : ELASTIC MODULUS =',E11.h,

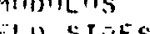

IF tita

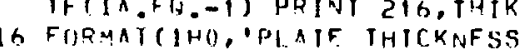

$=1, E 11.6,11$

CHECK AVATLABLFE BTIJRASE

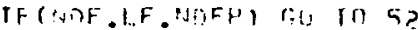




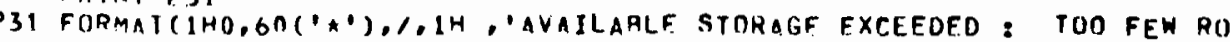
SWS IN ELASTIC MATKIX',1,61(**1)

52 IF (NAW.LF.NECH) RO TO 53 PRTAT P3?

?3? FORMAT(1H0,63( $\left.{ }^{\circ}\right), 1,1 \mathrm{H}$, AVAILARLF STRRAGE FXCEFDFO: TON FEW CO SI.UMNS IN ELASTIC MATRIX?,

$\operatorname{sins}$

33 TH (NN.LE .NAP) BO TO 54 PRTNT 233

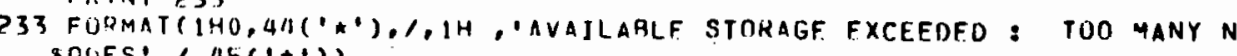
Sn!tes? $, 1,15(\cdot *+1)$

SITP

PQTNE.LE.NFP) GO In 55

PRTNT 234

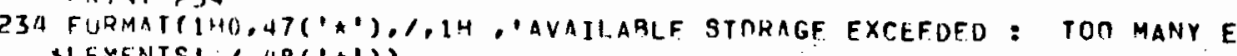
S1.. NENTS $, 1,4 R(\cdot * 1))$

If

TF (IS.LE.ISP) GO TO 10

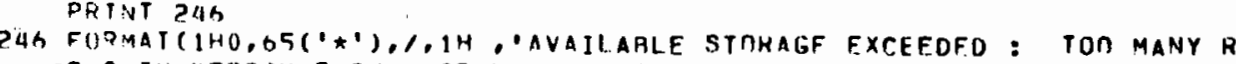
DINS TN DEFORMATION MAIRIX0,1,6h('*'))

c. pRINT nOOAL COUROINATES

10 IF (IA.LT.1) PRTNT ?OA

OOA FORMAT(1HO,'COORDINATES OF NODES',1/,' NODF',6X, 'X',11X,'Y', S $3\left(10 x_{0}\right.$, WODE', $\left.\left.6 x^{\prime}, X^{\prime}, 11 X^{\prime}, Y^{\prime}\right)\right)$

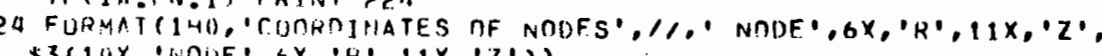

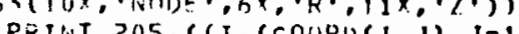

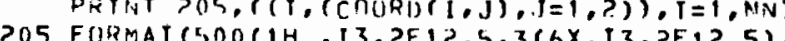

PRINT ELTMENT INCTOFACES

PRTNT PUG

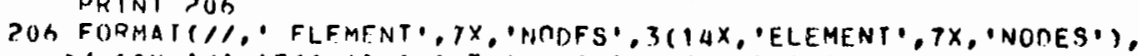
B $10 x, 1,13(\cdot-1), 1,1,3(14 x, 1,13(1-1), 1,1), 1)$

PRTN $207,((1,(I F L T(1, J), J=1,3)), I=1, N E)$

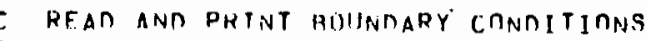

IF(IA.LT.1) PRIAT 208

208 FOPMATII, ROINHARY CONDIIIONS: 0 - CONSTRAINEDO

$\$ 1,74 x, 11$ - UNCONSTRATNED', 1,1 NODE $, 3 x, \cdot x, y \cdot, /]$

IF(1A.FQ.1) PRINT ?25

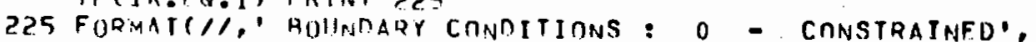

$5, ? 4 x, 11$ - UNCONSTPAINFD',1, NONE',3x,'R,2',11

ni) $1 \quad 1=1, i^{\prime} \cap F$

$1 \operatorname{tar}(t)=1$

7 Pt. $\Delta$ (i) 100.1

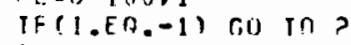

QEAD) 0,100$) \operatorname{TIRC}(1), \operatorname{TIAC}(2)$

FEA 1 i) 1 (n), I

$3 k=k+$ 


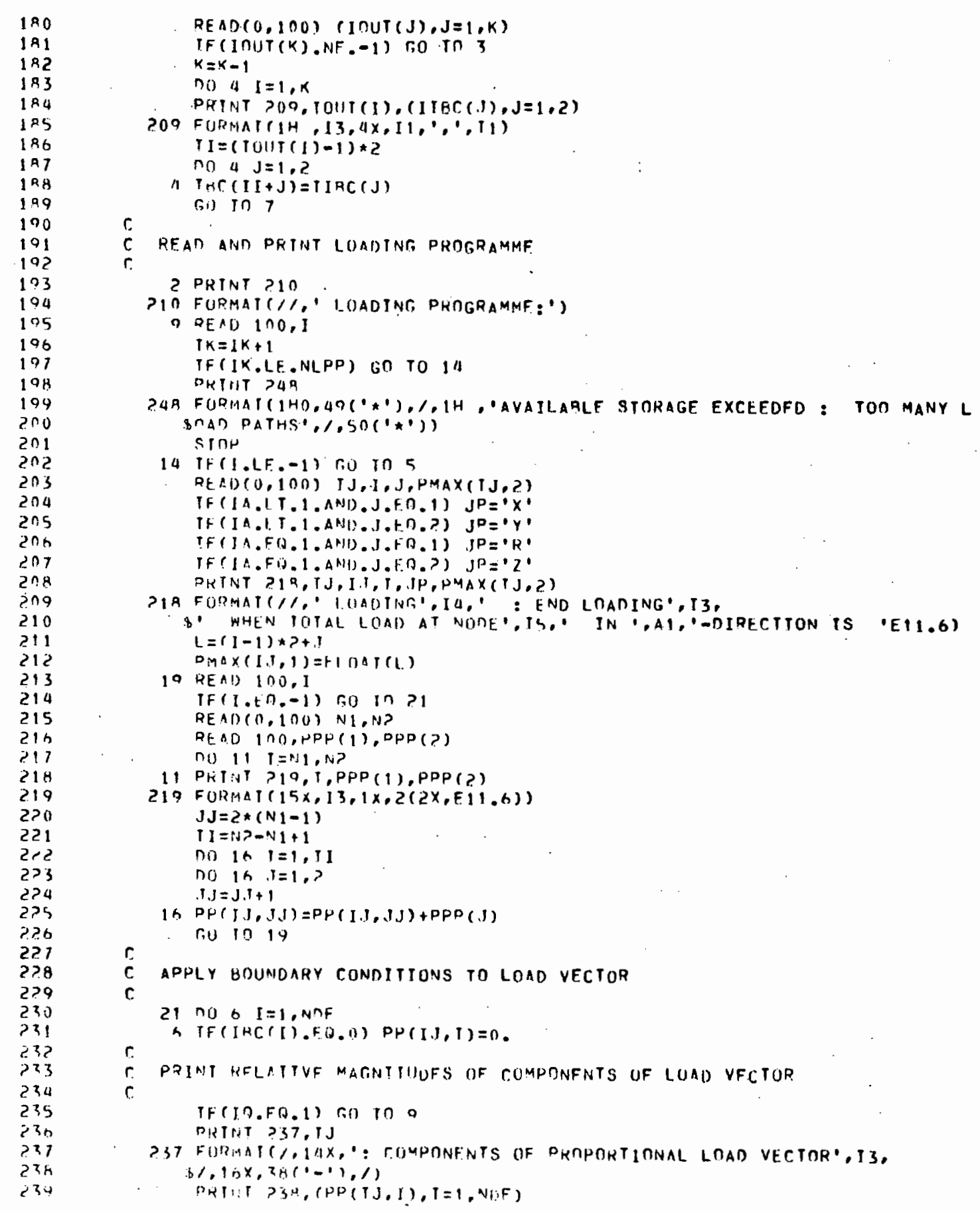



Go Tn 0

\section{REAT ANT PRINT DUTPUT REgUESTFD}

\section{PRTNT 230}

39 FORMAT(/,1HO, REDUFSTED DUTPIST OF RESULTS :-1,1,30(1-1),1) READ $103, J \mathrm{JP}$

103 FURMAIIAP)

$$
\text { TF(JP.NE. 'AL') GO TO } 23
$$

NO 59 TE $=1$, NF

NEL.TPR $(I F)=1$

Do $20 \quad T=1 . N$

MUNEDRTIT=1

DKTNT 2 \&I

?.4 FORHAT PRINT KFSULTS FOR ALL ELEMENTS AND ALL NUNES',

23 DEAD 100.

TF (I.ED.-1) GO TO 26

$33 k=k+1$

READ $(0,1 \cap B)($ I UUT $(.1), J=1, K)$

It $(1$ INUT $(k) . N F,-1)$ f,o in 33

$k=k-1$

ni) $25 \quad t=1, k$

(

DRTNT 241

241 FODMATCIN, IPRTNT RESULIS FOR FLFMENTS :-

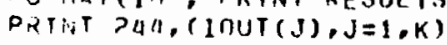

2h PFAD 100,1

IF $\left(1\right.$, t. $\left.R_{-}-1\right)$ (6) in 30

$x=0$
$x=x+1$

$x=x+1$

PEA $(0,1 \cap 0)$ (InUT $(J), J=1, k)$

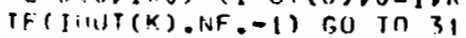

$k=k-$

$\operatorname{lig}_{1=1} 27 \quad I=1 \cdot k$

27 NONEPK $(j)=1$

DRINT $? 43$

243 FODMAT( 1 HO, 'PRINT RESUI.TS FOR NODES :- $)$

PRTET ?U/4, (IDUT (J), J=1,K)

244 FIMMAITH, 3314$)$

3ก DEAD 100.1

$k=1$
$k=k+1$

READ $(0,100)$ (INUT $(J), J=1, K)$

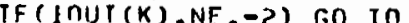

PRTNT PQS

>4S FURAAT(140, DRESULTS PRINTEN AT END OF FACH PROPORTIONAL LOAD PATH AidT AFTE? L.BAL INCREMENTS :-') 
CULAFPTCS(1).ELTMAT

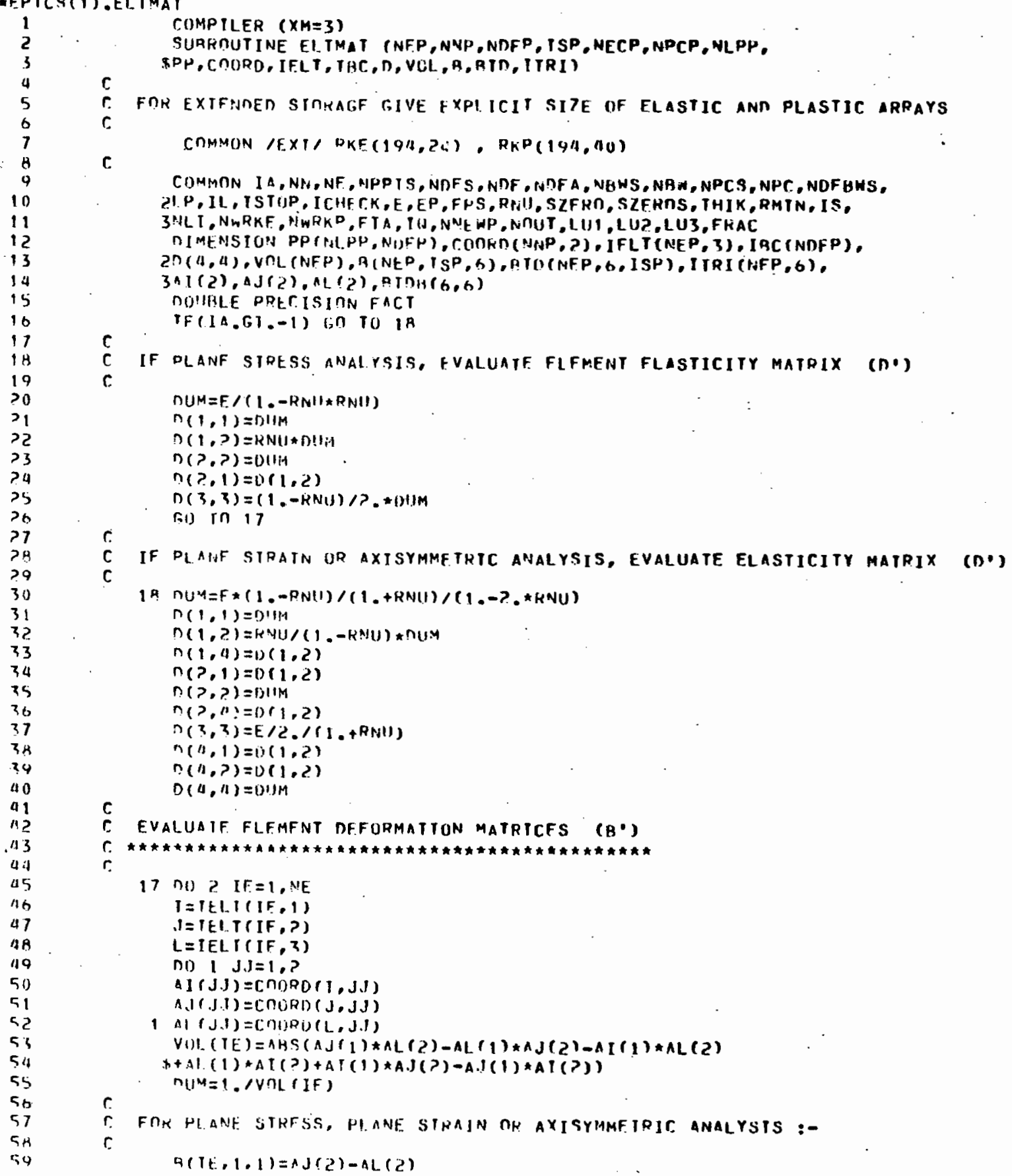




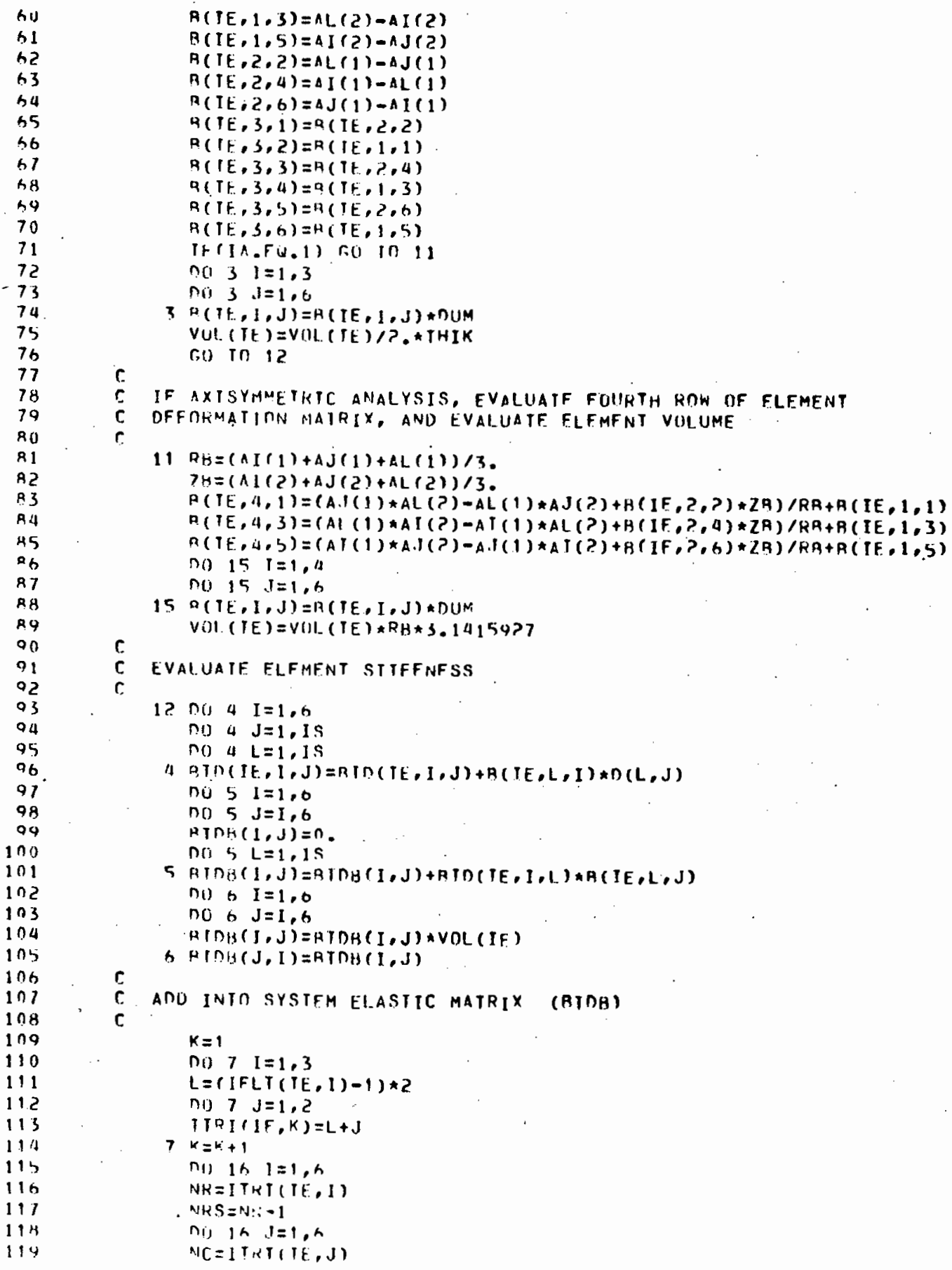


no $A \quad 1=1$, NHF

IF(IACCI).FQ.1) GO TO A

ni) \& $\mathrm{J}=$ ?, NQin

$9 \operatorname{PKF}(T, T)=0$.

$\operatorname{PKE}(I, 1)=1$.

If (I.En.1) Go to 8

$I I=I-1$

$I K=$ ?

$\lambda J=I-n B H+1$

TF(J.T.LT.1) $J J=1$

no 10 IJ $\mathrm{J}=1 \mathrm{I}, \mathrm{JJ},-$

PKF. (IJ,IK) $=0$.

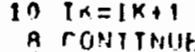

STORE HAIN TF SYSTEM ELASTIC MATRIX (BTDR) IN TEMPORARY FILE

CALL NTHANCLU1, 1, NWRKE, RKE,L, 2?

IF (L. FG. NWRKF) GO TO 27

PRTAT POZT,L

R2? FORMAT( ERROK UN WRITING SYSTEM ELASTIC MATRIX (BTDA)' S. : ERROH CODE TS , T4)

$c$

DFCOAPUSE EI.ASITC SYSTEM MATKTX (HTDR) INTO PRONUCT (UTAII)

27 nO $21 \quad$ I $K=1, N \cap F S$

$T H=18+1$

$T E R=T R+N A W S$

IF ( IAH.GT.NOF) IAH=NDF

$N C \cap L L=N H W S$

$i c=r$

DII $24 \quad I=I H, I R H$

$T C P=T C$

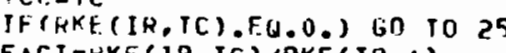

FACI I =KKE (IR, TC)/RKE (IR, I)

TF(IR, FST NNFRWS NCOLL=NDFA-

OKF (I,

FKF $(T, I)=R K E(I, J)-F A C T \star H K F(I R, I C C)$

$2 h \quad I C, C=T C C+1$

25 NCOLL $=$ NCOLL -1

4 $I C=I C+1$

FAC $1=D S$ SUR I $(\operatorname{RKE}(I R, 1))$

PKF $(1 K, 1)=F A C$

FAC, $I=1$. IFAC

MCOIL = MiB

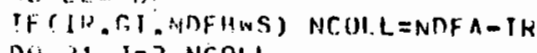

DO $? 1$ I $=$, NCNLL

$21 \operatorname{PKF}(T K, J)=R K F(I R, J) \& F A C T$

- PKE $\left.\left(N_{i}, 1\right)=\operatorname{SIJHT}\left(\operatorname{QKF}\left(N_{1}\right) F, 1\right)\right)$

C. STURE. HAIUN OF DFCOIPUSER SYSTFM FLASTIC MATRIX (II) TN TEMPURARY FILF 
$1 \% 0$

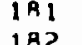

182
1 A3

184

1 R5

Iค7

IAP

CALL NTRANCLUZ, 1, NWRKE, RKE, , , 2?)

IF (L.EN.NWRKF) GO TO. 13

A21 FDRMATC ERROK ON WHITING, DECOMPOSFO SYSTEM ELASTIC MATRIX (U)?, \$ : ERROH COUF IS, 14 )

$\sin P$

13 PE TURN

END 
COL *EPTCS(1).SDLVE 1

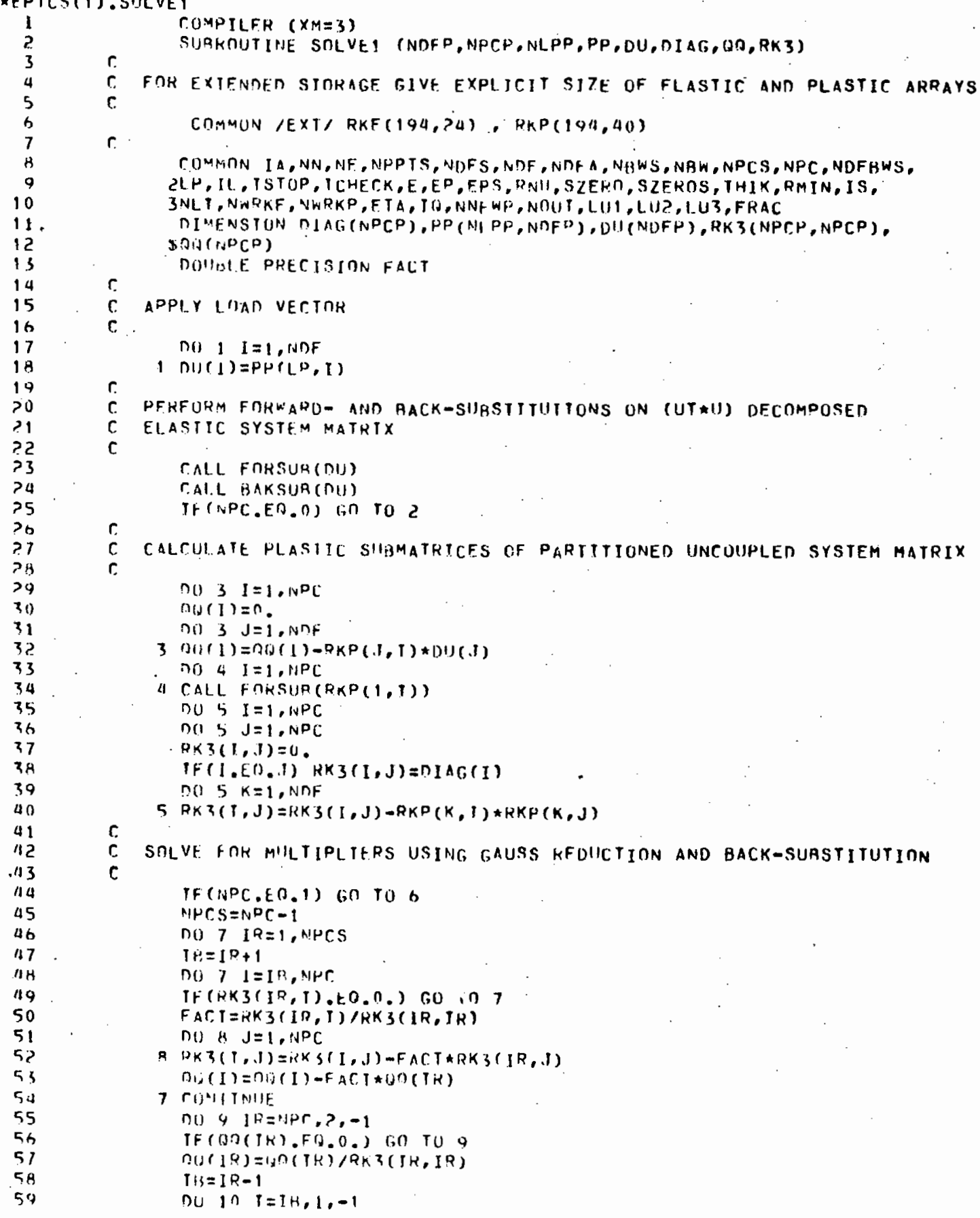




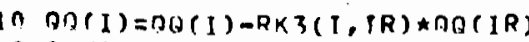

a CONTINUE

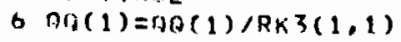

C RFAO PLASTIC MATHIX (-RTON) FROM TEMPORARY FILE

CALL NTKAPCLL13,10,?, NWRKP, RKP,L, PZI

IF(L.t T. NWPKP) GO TU 19

$$
\text { PKINT ALO, }
$$

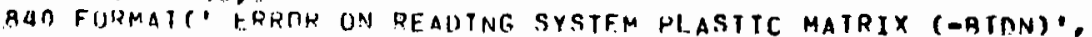

\$: : FQROKR COUE IS', I 4 I

c. CALCULAT

GALULAE RHS VECTOR OF PARTITIONEO UNCOUPLFO SYSTEM MATRTX

11 ก) $1 \geq T=1$, NDF

$n \in(I)=P P(L P, T)$

c.

a $15 . I=1$, Nite

SOLVE FOHE UTSPLACFMENTS USING FORWARD- AND BACK-SUHSITIUTION

$$
\begin{aligned}
& \text { CAIL FORSUR(DU) } \\
& \text { CALL GAKSUR(MUI) }
\end{aligned}
$$

$?$ RETUR:

C SIIHRUIIIINE FUR FURWARD-SUASTITUTION ON (UTAU) DFCOMPOSEN ELASTIC SYSTFM MATRIX

SURHOUUTINE F OLSUR (RHS)

TIMENSTUN RHS (NDFP)

NI) 1 IN $=1$, NUFS

IF(KHS IP).EO. .) GO IO

EHS $(T H)=R H S(T K) / R K F(T R, I)$

$I H=[D+1$

$T H R=I H A$ HAW

IF (IR:G.GT.NOF) [DB=NNF

$i=3$

QHS $(1)=1+1$

i rijet INIJE

QHS $($ HI) $)=R H S(N D F) /$ RKE $(N D F, 1)$

RETULN

C SHBPUIITIJF FUR AACK-SUBSTIIUTION ON (UTKU) DECOMPOSED ELASTIC SYSTEM MATRTX

SURKOUTINE BAKSUR (KHS)

TIMENSIUN RHS (NUFP)

no 1 IR=NDF, $?,-1$

TF(RHS(IR).EO. O.) GO TO

RHS $(T H)=R H S(T R) / R K F(T R, 1)$

$T B=1 B-1$

$T H A=T H-H A W S$

IF (INB.LT.1) I HAL =

ni) $? \quad 1=14, T$, IR, -1

BHS $(1)=\operatorname{RHS}(1)-\operatorname{RKF}(I, J)+R H S(I R)$

? $t=3+1$ 


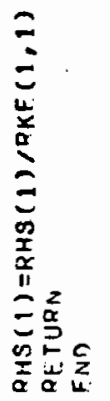

ำก 
CULAEPTCS(1).SOLVE?

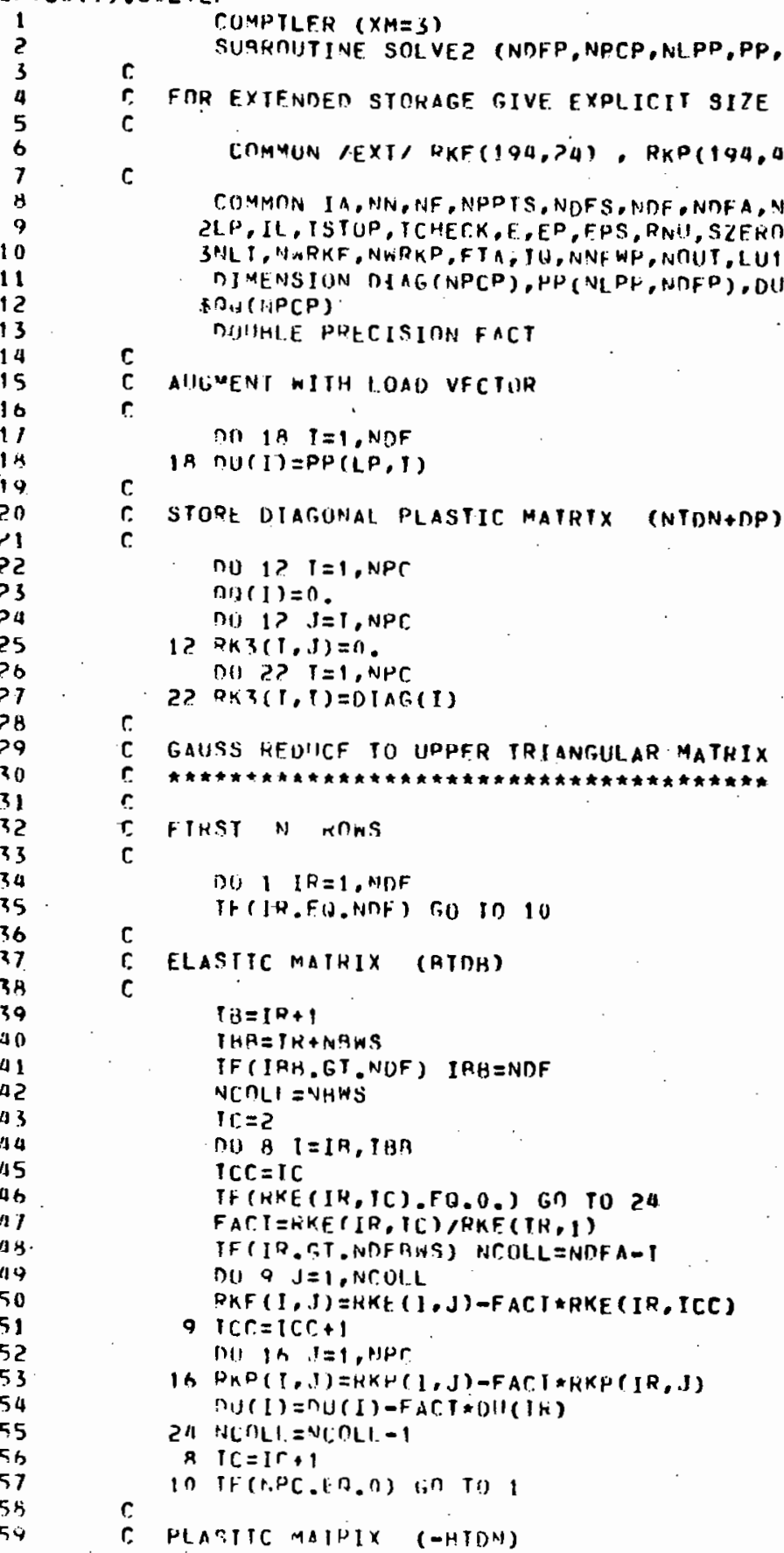


A-56

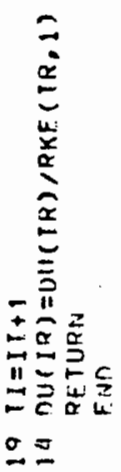

포ำ 
SP. I, FSTRN, PSTRN, STRN, STRS, SITEARR,PP, TELT,PMAX, TUIIT,

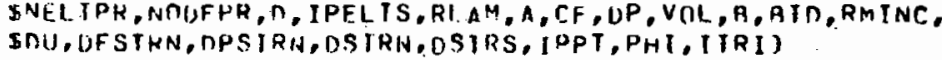

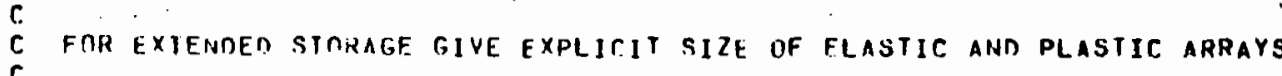

COMMON IEX.1/ RKF(194.74), RKP(194,90)

COMMON IA, NN, NF, NPPIS, NOFS, NRF, NOFA, NBHS, NRW, NPCS, NPC, NDFEWS

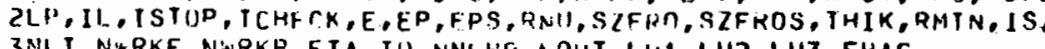

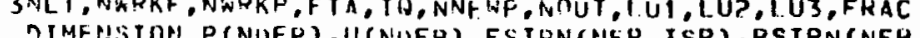

2SIIRNE IFP, ISP), STRS (NFP, ISF) SIOUAR (NFH, ISP)

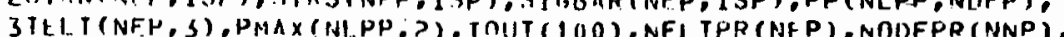

$4 T(4,4)$, IPF IS(NEP, 3), RLAM (NEP)

SUO (NEP), G (NFP, ISP, OR) R TD (NP

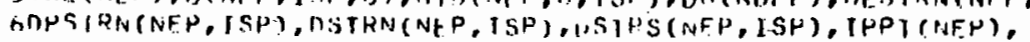

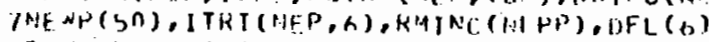

numbt PRECISINA OIIM

$\stackrel{c}{c}$

FOR LACH ELFMFINT :-

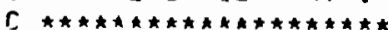

nO. 3 I $F=1$, NE

C CALTULATE KFLAITVF MATNITUDES DF TOTAL STPAIN INCREMENT

no $1 \quad 1=1.6$

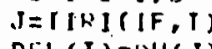

DEL $(T)=1) 11(.1)$

nII 2 IR $1=1$, IS $(T E, 1)=0$

Dut $5=1,0$

PSTHN (IE., I )=RSTRN $(T E,, 1)+R(I E, I, J) * D E L(J)$

If (IPHLLS(TE, 1).EQ.1) $\mathrm{r}, 0$ TO 5 .

C. FOR ELASTIC EIEMENIS r.ALCIILATE RELATIVE MAGNITURES OF ELASIIC STRAIN

AND STKFSS INCKFMENT VECTORS. AND SCALAR MULTIPLIER TO CAIISF STRESS

POIMT IO RE.ACH YIELA SUIRFACF. FROM ALL FLASTIC ELFMFNTS DFIFHMINE

SMALLFST SCALAR MIJLTIOLLTER

no 4 $1=1,15$

TESTRU (IF, T)=OSTRN(IF, I)

NSTKS $(1 E, I)=0$

no) $4 \mathrm{~J}=1, \mathrm{IS}$

$\operatorname{nSTRS}(I E, I)=\operatorname{nTTS}(I E, I)+n(T, J) * D S T R N(I F, J)$

C.ALL ROUT(DUM, RSTRS(TE, 1), DSTRS(TE, 2), RSTRS(IE, 3), DSTRS(IE, 4),

ni) $=011 \mathrm{M}$

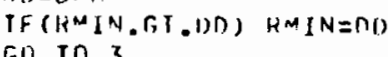

c

FOH MIASITC ELEMENTS CAICILATF PELATIVE MAGNITUNES OF ELAGIIC STRAIN,

PLASIIC STKAIN, ANI) STRFSS IAR M.FT.FNT VECTORS 
DO $6 I=1$, IS

DPSTRN (IF, I) =RLAM(IE) $\star A(I E, 1)$

$a$ RESTRN (IF, I ) =DSTRN (IE,T)-DPSTRN(TE,I)

no $7 \mathrm{I}=1,1 \mathrm{is}$

nSTKS $(T E, I)=0$.

Di $7 \mathrm{~J}=1$, IS

7 nSTHS $(T E, 1)=D S T R S(T E, 1)+n(T, J)$ RDESTKN $(I E, J)$

C

3 CUNTTNUE.

CHETK IF PRIMPTSEO MAGNITUNE OF TOTAL LOAD IN CURHENT LOAD DIRECTION

TITS LOANING PRUSRAMME

\section{IR= (FIX(OMAX(LP, 1))}

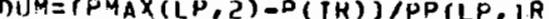

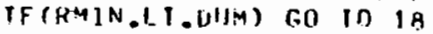

RMTN=i)HM

c

A RMINIC (LP) $=$ RHINC. $(L P)+R M I N$

MULTIDLY ALL INCKFMENT BUANTITIES GY SMMLLESI MILLIPLIER, AMIJ IETFRMINE

CINRENI IOTALS OF LOADT, DISPLACFMFHT, ELASII, STHAIN, PLASIIC STRAIN

ANI) STHFSS

ni) $17 \quad I=1, N(j)$

nU(I) $=$ nU(I) $\star R M T N$

II (I) $=! !(I)+n U(I)$

$n+P(1)=D M T N+P P(1, P, 1)$

$17 P(I)=P(I)+n P(I)$

ND 11 IE $=1, \mathrm{NF}$

nil $\quad$ i $\quad l=1$, is

חSTRM $(T E, J)=n S T R N(T E, J) \star R M T N$

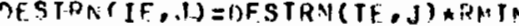

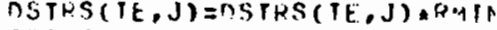

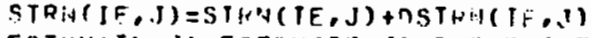

FSTRN(TE,J)=FSTRN (IE,J) D DFSIRN IF, J)

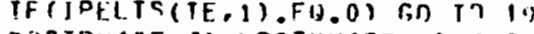

RPSTRN $[F, J)=$ U) PSTHN(TE,J) APMTH

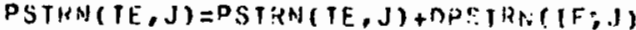

19 SIUS $[F, . J)=S T K S(J E, J)+D S T H S(T F, . j)$

12 SITBAK IF, JI)STRS(TE,J)-FPNPSTRM(IF,J)

TF(IPETIS(TE. T).FO.O) GO in 1

$c$

PLAM(IF) $=R L A M(I F) \star R_{H A N}$

IN FLASTIC-PERFECTLY PLASTIC CASF, NEUTRAL-LOADING STRESS POINTS

MIVF TANGENTIAL IO YIFLD SURFACF, THEREFORE CORRECT CURRENT

STKESSES AY KETUKNING STKESS POTNT TO YTELD SUHFACE

\section{TFACT $=$ ?}

CALL ROUT (DUM, STRS (IF, I), STRS(IE, 2), STRSTIF, 3), STRS(IE, 4$)$,

\PSTKN(TE, 1),PSTRN(IE, 2),PSTRN(IE, 3),PSTRN(IE, 4))

C.F $(I F)=011 M$

no $14 \quad \mathrm{~J}=1, \mathrm{TS}$

14 $S I T H A H(F, J)=S T R S(I E, J)-F P * P S I R N(I F, J)$

$\stackrel{c}{c}$ 
No 15 IE $=1, N F$

TF(TPEITSCIE, 1).EO.1) Fo TO 10

TH(LA.GT.-1) GO TO 9

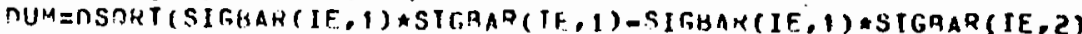

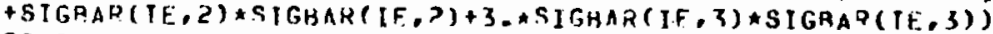

C,O 10 in 8

- NUM= ISNRT(SIGBAR (IF,1)*STGRAR(IF,1)+SIFHAR(1F,?

B*STGHAR (TE, O.2) +SIGHAK(IF, 4) *STGRAR(TE, (4)-SISBAR(IF, 1)

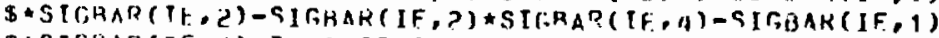

S*STGHAR $(T E, 4)+3 . \star S I G B A R(T E, 3) \star S I F+1 A R(I E, 3))$

IT (OIM.LT.FTA) GN TO 15

TPFLTU $(1 F, 1)=$

NNF: $\triangle D=A M P F N^{P}+1$

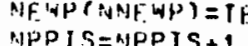

TPPT ( NPPTS) $=$ IE

12 cuntiontitis

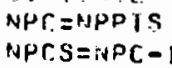

C. CHECK IF I.OAD INCREMENTS AECOMING CONSISTANTLY LESS IHAN PFRAC OF TOTAL LOAD

c

IF (EP.ST,0.0) G,0 Tn 20

IF(HMIN/PMINC(LP).FT,FRAC) GO TO 21

$N K N T=K K N N^{\top}+1$

IFINKAT.1.T.3) R.U TO ?O

DRINT PO1, FHAC

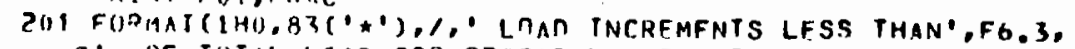

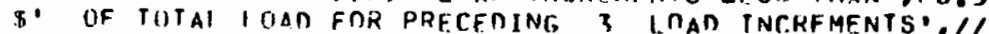

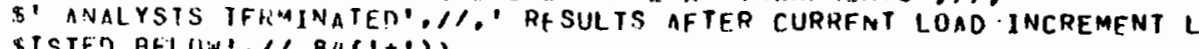
SSTE' PET(I)H', $1,84(\cdot * \cdot)$

ISTUP $=-1$

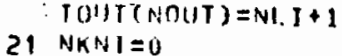

c.

DUTPUT CURKFNT DUMNTITIES

20 C.ALL OUITPUT (NEP, NNP, NDFP, ISP, NECP,NPCP, NLPP,

SDSTRN, SIRN, STRS, SIS.HAR,PMAX, TOHT, NFLTPR, NODEPR, KIIIVC.

\$PHI, IPFLTS, RL AM, CF, DP, NU, DESTRN, OPSIPN, DSIRN, DSTRS, IPPT

$\$ P, I I, F S T R M, N E W P$ )

r.

If (ISIOP.ER.-1) GO in 16

NWRKP $=$ NDF $\star N P C$.

IF(NPE.LF. APPCP) GU IO 13

DRTNT >OD?

20? FORTAT(1HO,SA('*'),1.' AVATLABLE STORAFE EXCFEMEN : PLASTIC MATRI

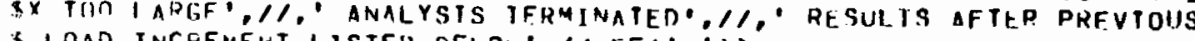

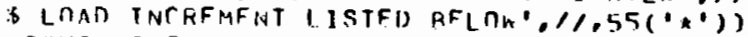

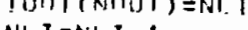

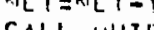

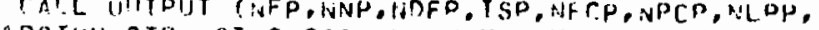

SDSTKN, STRN, STKS, SISHAK, PMAX, TUIT, NFLTPR, HGOPER, RMIVC, 
13 TF (NPC.LF.NBW) $L I U=L U$ ?

T.F (NPC C GT NHW) $L I I=1 U 1$

C.ALLL NTHAN (LII, 10, Z? ? NWRKF, RKF,L, Z2)

TF(L.EN.NARKF) GO TO in

PRTNT Aट̃O,

AZI FORYATI' ERRTR ON READINT, SYSTFM ELASTIC MATRIX (BTDR)'. S.: ERROH $\left(\cap_{1}\right)$ F. IS, 14$)$

C SIGROIIIINF FOR TFTFOMINING SCALAR MULTIPLIFRS FOR ELASTIC ELEMENTS

C AHU FOK RFTIIRNIMG; STRFSS POINTS TO YIELO SURFACF FUR PLASTIC FLEMENTS

SIIRROUTINE ROOT $\left(R T, X_{1}, X_{2}, X_{3}, X_{4}, Y 1, Y 2, Y 3, Y 4\right)$

NUIIHLE PRECISION AA,RH,CC,RT

TF(IA.GTE-1) GA TO

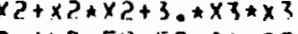

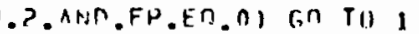

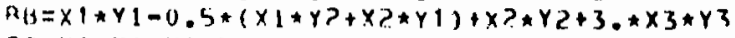

$r C=Y 1 * Y 1-Y 1 * Y Z+Y P \star Y Z+3 * \star Y Z * Y 3$

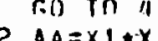

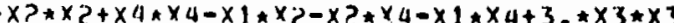

TF(IFACI.ER.?.ANA.FP.EO.n) GO TO

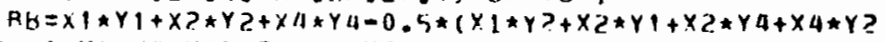

$\$+X(* Y 4+X(1 * Y(1)+3 \ldots * X 3 * Y 3$

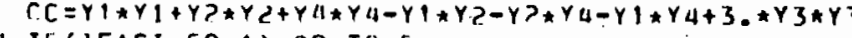

TFEIFACT.CO.1) GO TO 5

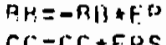

$5 R I=(N S A K T(A B \star H R-A A *(C C-S) E R O S))-A B) / A A$

RE TURN

I RI =STERO IOSURI (AA)

RE TURN 
COL \#PTCS(1).PLASIH

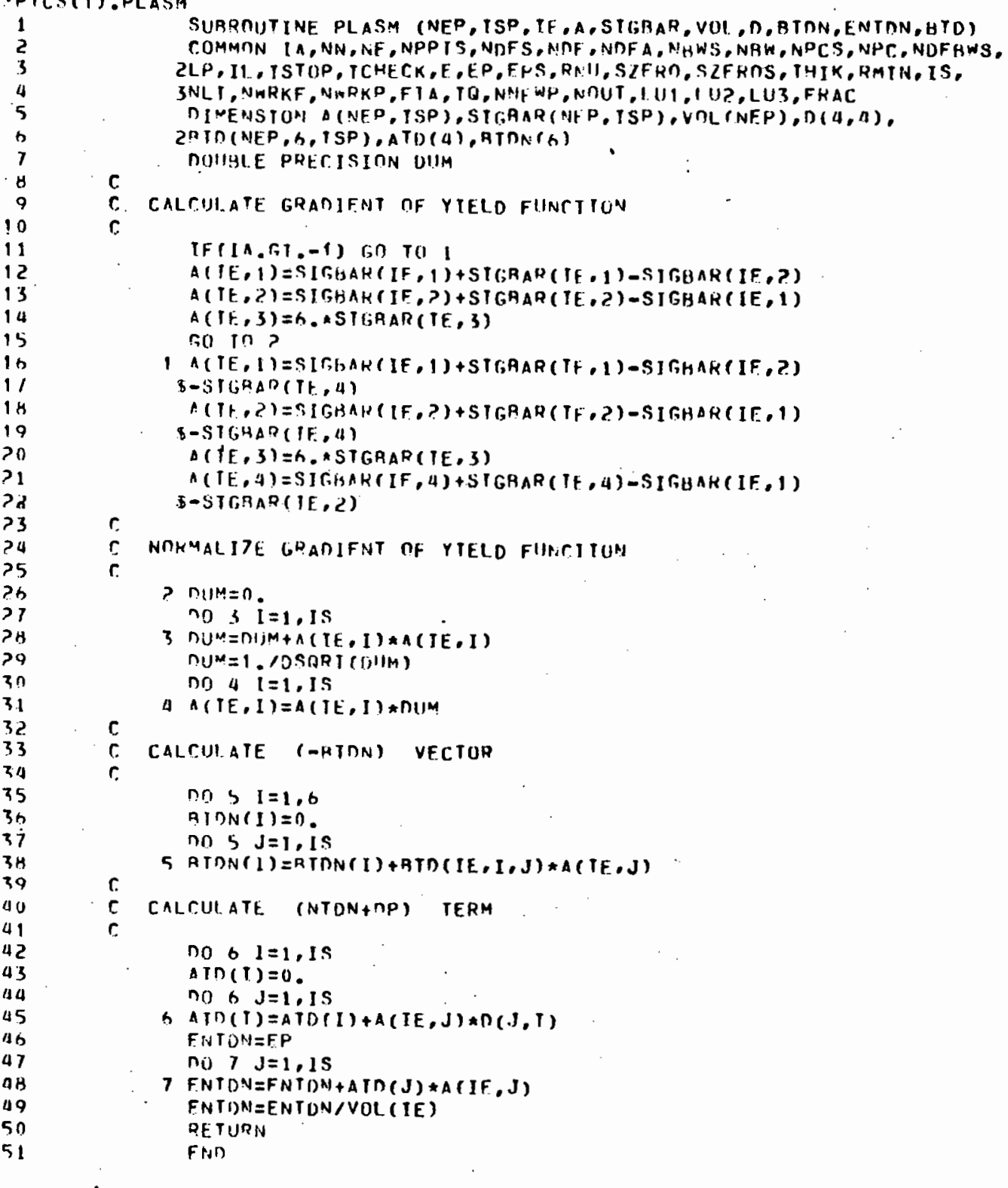




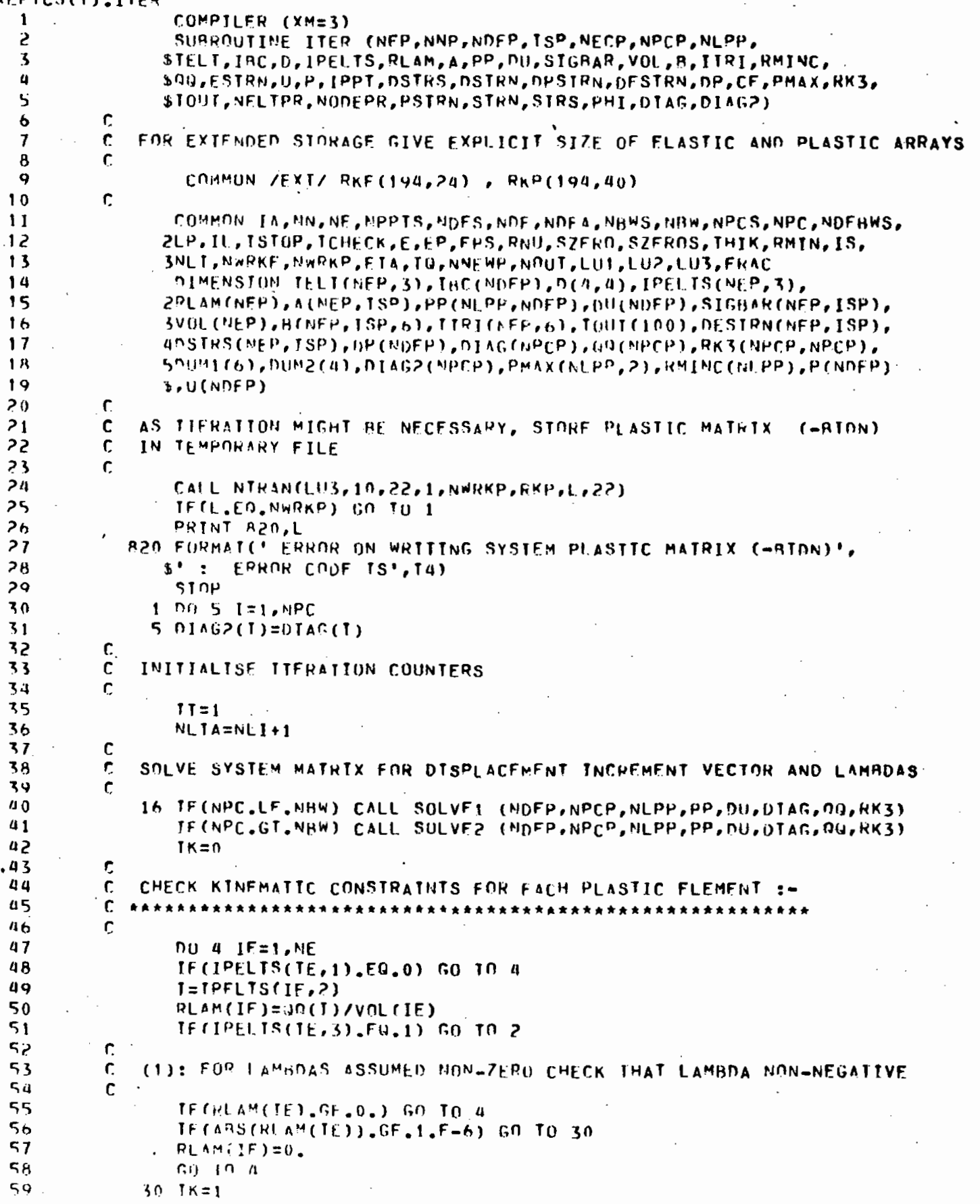


(?): FUP LAMHOAS ASSUMED 7ERO CHECK, THAT SCALAR PRUNUCT OF GRADIENT OF YIELO FUNCTION AND NORMAL. ILFD STRESS INCREMFNT VECTOR NON-POSITIVE ELEMENT NOUE MISPI.ACEMENT INCREMENTS

$$
\begin{aligned}
& ? \text { no } 6 \quad I=1,6 \\
& J=T r R I(I F, T) \\
& \text { nUMMI }(1)=n(j(s)
\end{aligned}
$$

CALCIII.ATE RFLAITVF MAGNITHDFs OF COMPONFNTS OF FLASTIC. STRATN

INCREMENI VECTOP

$$
\begin{aligned}
& \text { no } 13 \quad I=1 \text {, Is } \\
& \text { nym } 2(I)=n \text {. }
\end{aligned}
$$$$
\text { nil } 13 \quad 1=1,0
$$

(I) $+R(I E, I, J) * n U M I(J)$

c

CALCIILATE RFLATIVF MARNTIIDFS OF COMPONENTS OF STRESS INCREMENT VECTOR

$$
\text { กत } B \quad I=1 \text {, Is }
$$

no 8 \& $j=1$, is

$1, . J) * 0.193(.1)$

C NIRMALIIZE RELATIVE MAFNITIIDFS OF COMPONENTS OF STRESS INCREMENT VECTOR חUM=n.

no $10 i=1$, is

in $n U M=n(J M+n U M 1(1) * \operatorname{nUM} 1(I)$

$n U^{M}=1 . / S \cap R T(\cap U M)$

no 1 ? $i=1, T S$

C CALCULATE AND CHECK SCALAR PHODIICT OF GRADIENT. OF YIELD FUNCTION AND NOKMALIZED SIRESS INCREMENT YECTOR

num $=n$.

num $=n$ i $i=1,1 \mathrm{~s}$

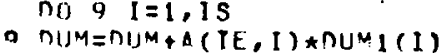

TF(D) HM.LT.N) GO TO 4

$$
T K=1
$$

TPELTS $(I F, 3)=0$

PR?NT $202, N L T A, I T, T E, D U M$

2. FIFMFMT

\$ FLFMFAT

i) ruIINIIE

IF IIFKaITON cIONSTHAINTS SATISFTED, CONTINUF

IFCIK.FO.0) r, i in 12

r. IF TIFRATIUN PROCFSS NUT CONUFHGING TFRMINATE ANALYSIS 


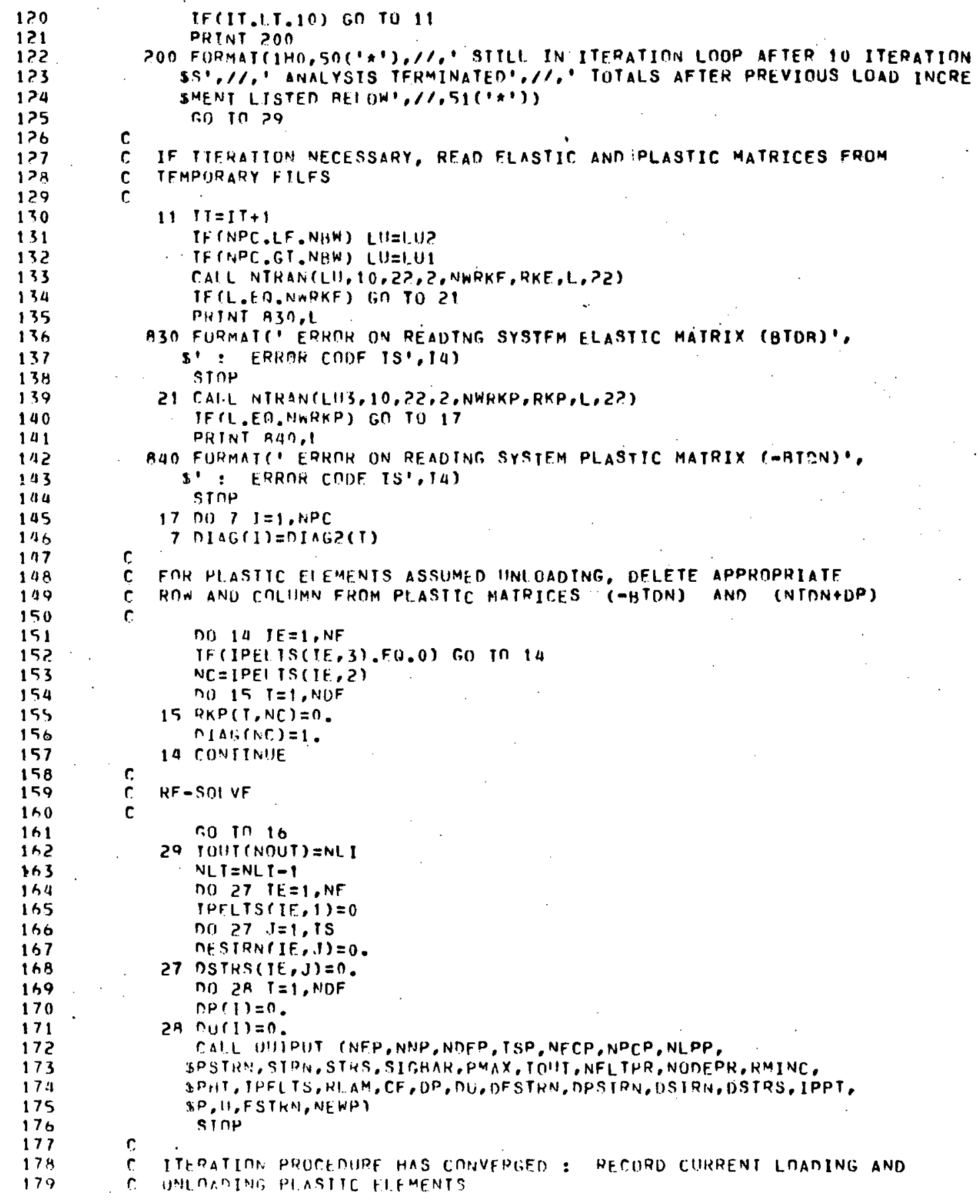


A-65

s

¿

$\dot{0}$

4

mo

造高落

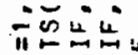

酷的立

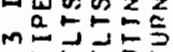

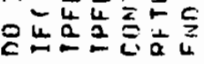

$\simeq$

c

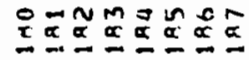


SURRDUTINE OUTPUT INEP, NNP, NIFP, TSP, NECP, NPCP, NLPP,

SDSTRM, STRN, STRS, SIGHAR, PMAX, TOUY, NFL LPR , NODEPR, RMINC,

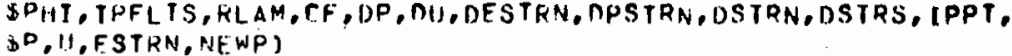

\$P, G, ESTRN, NE.WP )

作

21.H. I1, ISTUP, ICHECK,E,HP,EPS, PNII, SZFRT, SLFROS, THIK,RMIN, IS,

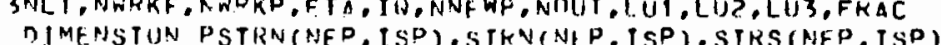

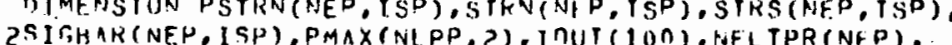

3NODEPK (NNP), PHI (NEP) IPFL IS (NFD, 3 ) KL AM (NED) CF (NEP)

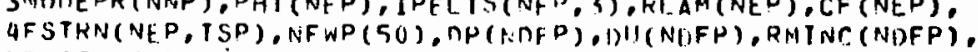

SMESTRN (NFP, ISP), DPSTRN (NEF, ISP), DSTRN (NEP, ISP),

ONSTKS (NEP, TSP), IPPT (NEP),P(NDFP), U(NDFP)

NL. $T=N L T+1$

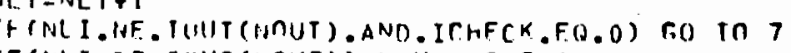

C PRINI HEANINGS

IF (NLI.ED, TOUT (NOUT)) NOUIT=NOUT +

C PRTNT POO,NLI

200 FORMAT(IHI, 'RESUT IS AFYER', 14, PX, 'LOAD INCREMENTS', 1)

TF (ICHFCK.FO.1) 60 TO 1

, NNEWP )

?.01 FORMAT(1HO,NEY FLEMENTS TO UNOERGO PLASTIC DFFORMATION :, 10.15 $6(11.47 x, 1015))$

$I=\left(I F I \times\left(P_{M A} \times(1, P, 1)\right)+1\right) / 2$

FORMAT

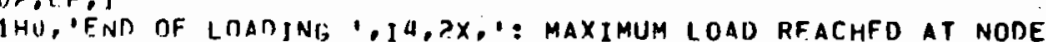

S. $\cdot$. T4

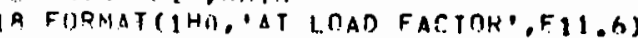

no $6 \quad 1=1,2 P^{2}$

$\operatorname{rinc(t)~}$

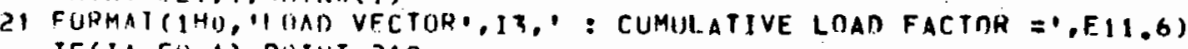

TF (IA.FA. L) PRTNT 219

219 FURMAT(1HO, 'NOTE: CIRCUMFERENITAL STRAINS AND STRESSES ( $(1)$ CORRESP SANH TO CENTKOINS OF ELFMENTS', $\left.1,1 H^{+}, 44 X, 1-1,1,6(1-1)\right)$

TF(ISHFCK.FO.0) GO TO $>3$

$1 P=L P+1$

TCMECK $=0$

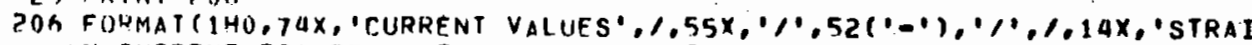
SN TNRREMFNTS', 37X, 'STRAINS', 38X, 'STRFSS', /,12X, $1,1,1$ (1'-1), 1, 14X

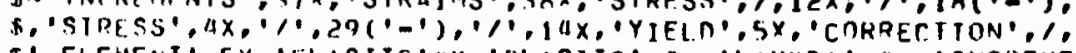

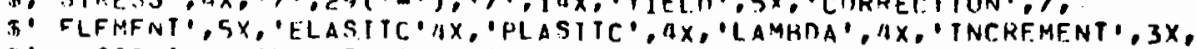
\$'ELASIIC',AX,'PLASTIC',5X,'THIAL',6X, 'SIRESS', 3X, 'FUNCTION',5X,

S'FACTUR', /)

C EVALUATE YIFLD FUNCTION FOR FI.EMENTS REDUESTEO AS UUTPUT

nU 4 IF $=1$, NE

NE. () GII In $n$

(TH

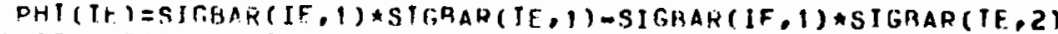

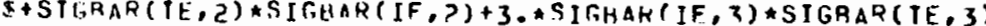

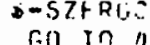

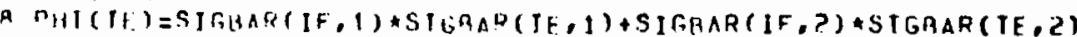


\$\$3, *SIGUAR $(I F, 3) * S I G R A R(I E, 3)$-SZF.ROS

PRT ELEMNT OHANTITIES FOR NUTPUT RFOHISTFO

If (IA) $9,13,10$

c Plane strfoss analysis:-

- NO 1? TE=1,NF

IF(NFL TPP(IE).NF.1) G,O TO 12

IF (IPELTS(IF, i), FO. O) r,o In 3

\section{PLASTIC ELEMENTS}

DRINT ?O7, DESTRN(IF, 1), OPSTHN(TE, 1), OSTHS(TE, 1), ESTRN(IE, 1),

SPSTKN(IE, 1), STRM(IE, 1), STRS (IE, 1), IE,DESTRN (TE, 2 ) OOPSTRN (IE,?),

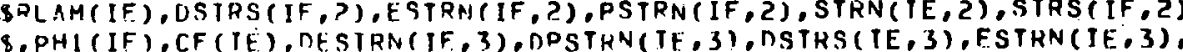

SDSTHN (TE, 3), STRN (IF, 3), STRS (TH, 3)

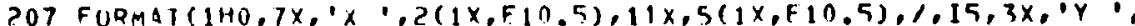

S10 $(1 X, F 10.5), 1,7 x, 1 X Y,, ?(1 X, E 10.5), 11 x, 5(1 X, E 10,5))$

rio $\ln 12$

$c$

FIASTIC ELEMENTS

3 PRTNT 205, NESTRN(IF, 1), DSTRS(IF, 1$), E$ STRN(IF, 1$)$,

\PSTHN(TE, 1),STRN(IF, 1),STKS(IF, 1), IE,OOFSTRN(IE, 2),

SISTRS(1E, Z), FSTRN(IE, 2), PSTRN (Tt, , $), S T R N(I E, ?), S T R S(T E, 2)$,

SOHI (IE), DESIRN IF, 3), DSTRS (IF, 3),ESIRN IF, 3), HSIRN (IF, 3),

SSIRN (IF, 3), STRS (TE, 3)

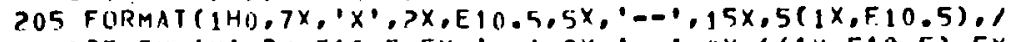

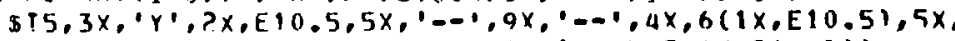

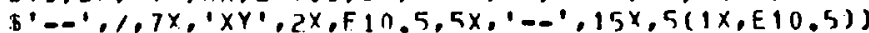

15 rOATINHE

c. Plane sthain analysis :-

rat In $>0$

13 NO $21 \quad T E=1, N E$

TF(NELTPR(IE).NE. I) GO TO ?I

TH(IPELTS(TE, i).EN.0) Go in 14

c

PLASTIC HLEMENIS

PHINT 212,DESTRN(IE, 1),DPSTRN(IE, 1),DSTKS(IE, 1),ESTRN(TE, 1),

\$PSTRN(IE, 1),SIRN(IF, 1),STKS(TE, l), TE,OFSTRN(TE,?),DPSTRN(IE.,?),

SPLAM(IF),DSTRS (IF, 2),FSTRN (1F,? ),PSTRN(IE, ?), STRN(IE, 2), STRS (IF, 2)

\$, PHI (IF), CF (TE), DF STRN (IF, 4), DPSTRN(TF, 4), NSTHS (It , 4), FSTRN(IE, 4),

SPSTRN(TE, 4), STRN (1F, " ) , STKS (TE, 4), DE SIRN (IF, 3), DPSTRN(IE, 3),

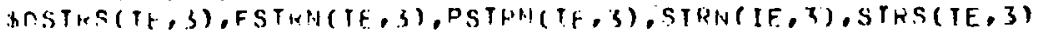

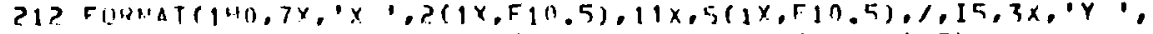

\$10(1X,F10.5),1,Bx, 2 $, .2(1 x, 510.5), 11 x, 5(1 x, E 10,5)$,

$\$ 1,7 x, \cdot x y \cdot .2(1 x, F 10,5), 11 x, 5(1 x, F, 10,5))$

6.0 In $>1$

ELASTIL FI EMENTS 
14 PRINT 213 , RESTRN(IE, 1), DSTRS(IE, 1), ESTRN(IE, 1),

SPSTRN(TE, 1), STRN (IF, 1),STKS(TE, 1), IE, DESTRN(TE, 2),

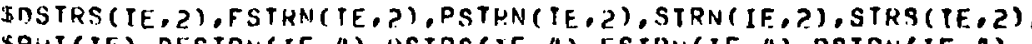

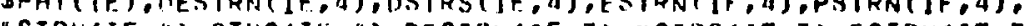
SSTRN (IF, 4), STRS(TE, 4), NFSTQN ( IF, 3), USTRS ( [E, 3), ESTQN (IE, 3),

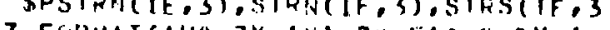

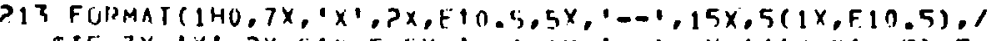

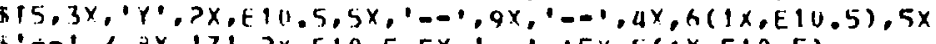
\$

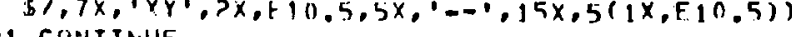

51 ron in the

r.u in ?

10 no 22 TE $=1, \mathrm{~N}$

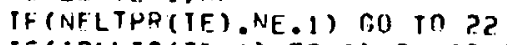

IF(IPELTS(TE, 1).FO.O) R,O TO

PLASTTC ELEMENTS

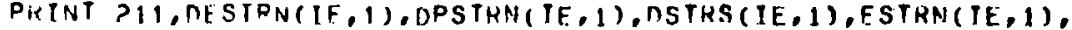
SPSTKN(TE, 1), STKN(IF, !), STRS( TE, 1), IE, OESTRN(TE, 2), DPSTRN(IF, ? )

BRLAM(IF), USTRS (IF, ?), ESTRN(IF,?), PSTRM(IF, 2),STRN(IE,? ), STRS (IF, ?)

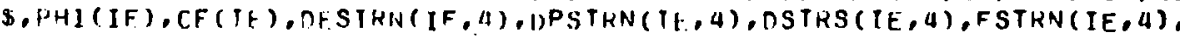

\$OSTKN (TE, 4), STRN (1F, 1), STRS(TE, 4), DESTRN IF, 3), DPSTRN(TE, 3),

\$NSTKS(TE, 3), FSTHN(TE, 3),PSTRN(TE, 3), STRN (IF, 3), STRS (TE, 3)

?11 FILMAT(1H0,7x, 'R , $2(1 x, F 10,5), 11 x, 5(1 x, F 10,5), 1,15,3 x, 17$,

$\operatorname{sio}\left(1 x_{0}, F 1 n, 5\right), 1,8 x_{0}, 0,02\left(1 x_{0}, F 10,51,11 x, 5\left(1 x_{0}, F 10,5\right)\right.$,

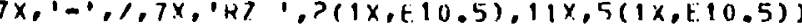

r.) In $>$ ?

ELASITC EIEMENTS

11 PRINT 214, DESTPN (IF, 1),DSTRS (IF, 1),ESTRN (IF, 1),

SPSTKN(TH, 1), STRN IF, 1),STKS(IE, 1), IE, DFSTRN(TE, 2),

InSTKS(IE, C), FSTKN(TE, 2), PSTKN(IE, ?), BTKN(IF, P),STKS(IE, R),

SOHI (IE), DESTRN(IF, I), OSTRS (IF, 4), ESTRN(IF, II), PSTRN (IF, 4),

SSIRNR IF, 1), STRS (IE, 4), DESTRN (IE, 3), USTRS (IF, 3), ESTRN (IF, 3)

\PSTHAI (TE, 3), SIRN (IF, 3), STRS (IF, 3)

214 F.ORMAI $\left(1 H O, 7 x, R^{\prime}, ? X, E 10,5,3 x, 1=-1,15 x, 5(1 x, E 10,5), 1\right.$

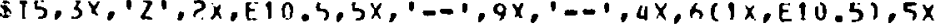

- , , $8 x, 0^{\circ}, 2 x, E 10,5,5 x, 1--1,15 x, 5(1 x, F, 1 n, 5)$,

5? rollithlle

C

C. PRINT CIIRRENT PIASTIC EIEMENIS

2N PRTNT 203

203 FORHAT $(1$ HO, 1,1 CLIRRENT PLASTIC ELEMENTS : $-1,1,27(1-1))$

IF(NPPTS.MF.O) GO TO 1 R

DKTHT >IO

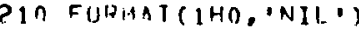

(C) 1019

IA PRTAT >IIL, (IPPT(I), I=1,NPPTS

204 FORAA1 (1H0,2515,1/,1n(1X, 2515,1/))

c. PRINI HFATINGS FUR MIGEE OHANTITIFS 


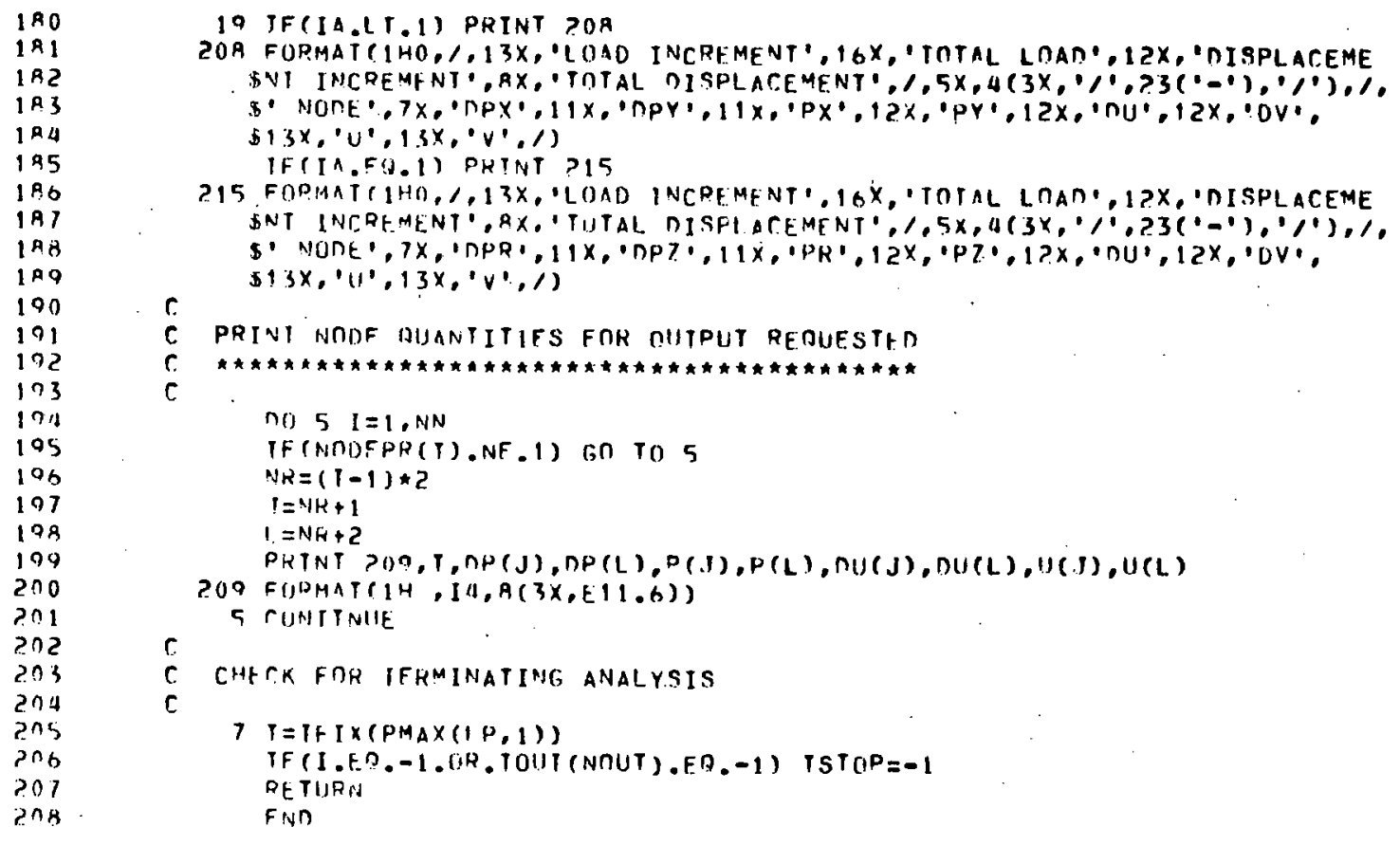


A -70

A.6 EPCQI Program Listing 
PARAMETER NMFP $=384$

PARAMETER NFCP $=$ R4

PAPAMETER NPCP $=30$

PADAMETER NNP = 19 ?

PARAMETER NEP $=31$

PARAMETFR INP $=3$

PARAMETER JSF $=3$
PAPAMETER NLPP = ?

$c$

COMMON /F.XT/ KKE (NOFP, NECP), RKP (NOFP,NPCP)

TUMMON IA; IO, NA, NE, NPPTS, NDIS, NDF, NUFA, NAWS, NHW, NPC, NPCS,

ZNOFHWS,LP, IL, ISIMP, ICHECK, F,FP,EPS,RNU,STERO,SZEROS, THIK,

GDIIN, IS, IUS, TOSM, NLL I, (FP $(1,4), W r_{1} T(4,4), N W R K F$, NWRKP,

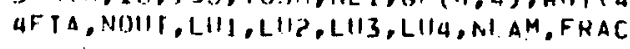

SET LOGICAL UNIT NUMGFRS TF IEMPORARY FILFS

$$
\begin{aligned}
& \text { LU1 }=11 \\
& \text { LUt }=12 \\
& \text { LU3 }=13 \\
& 1 \cup 1=14
\end{aligned}
$$

INITIALIZE ARRAYS

ПIMENSTON PP(NLPP, NDFP), CONRח (NNP, ?), IELT (NEP, 12), THC (NDFP), 2PYAX(NIPP,?), I NUT ( 100$)$, NFI. TPR (NEP, IOSP), NODEPK(NNP),

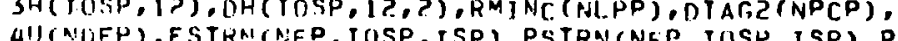

4II (NUFP),FSTRE (NEP, TOSP, ISP), PSTRN (NEP, TOSP, ISP), P (NOFP).

5STRS

6PLAM (NFP ,

GRYSTR O (NFP, IOSP N

US $Q$ (NTH

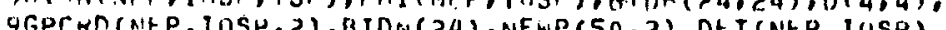

r. REAT STRUCTHRF TATA

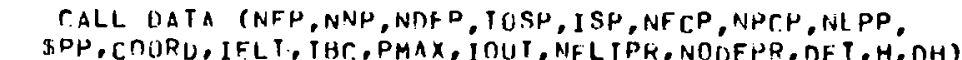

r. CALCUI ATE AND STURF IN TEMPORARY FILES, ELASIIC STIFFNESS MATRIX

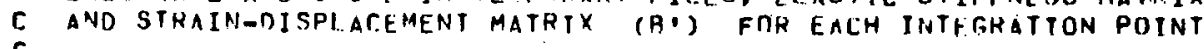

(BTDB)

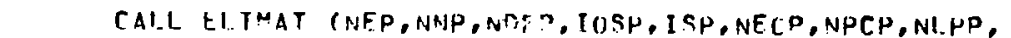

SPP, CNOPD, IFLT, TAC, D, DE T, H, D, G, GPRD, BTDRI

C INITIALIZF COUNTRES FOR FTRST LOAD INCRFMENT: LINEAR ELASTIC ANALYSIS

$1+=1$

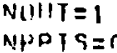

$\sin 0=0$

r. SILLV. FIK DISPLACFMFNT TNCPFMENTS 
1 CALL STRSTR CRFP, NNP, NRFP, TUSP, ISP, NFCP, NPCP, NLHP,

SP, "I, ESTKN,PSTKN, SIRN, STRS, SICHAR, PP, IELT, PMAX, IOUT,

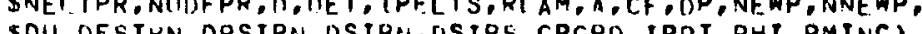

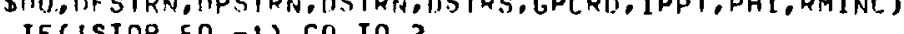

IF (IST)

TEMRORARY FILE IN WHICH INTEGRATION POTNT DEFORMATTON

MATRICTS (A') ARE STUREN

(ALL NIRAN(LI) , 10, ? $)$

c. EVALUATF PLASTIS MatKICES (-RTON) aND (NTON+DP)

$$
\text { ก) } 4 J=1 . N P C
$$

$n I A G(J)=0$.

no $4 I=1, N \cap F$

$4 \operatorname{RKD}(T, I)=0$.

Niw $=0$

Nhi $=0$

NO 5 IF $=1, \mathrm{ME}$

nO $S \quad I C=1,10$

TF(IDELIS(TE,IG, 1),EO.O) GO TO 5

$N=N+1-N \cdot N H$

CALL PI.ASM (NEP, IOSP, ISP, IF, IT, IPEL TS, A,SIGBAR,

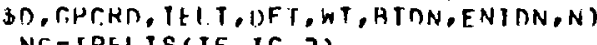

$N C=I P E L T S(I E, I G, ?)$

กn $n \quad I=1,1$ ?

NHR $=(1 F L T(T E, 1)-1) * 2$

NRF $=(1-1) \star C$

no $0 \mathrm{~J}=1$, ?

h DKP (NAR +, NC $)=\operatorname{RKP}(N R R+I, N C)-R T D N(N R F+J) \approx W T$

DIACi $(N C)=D T A C=(N C)+F N T D N A W T$

c

C APPEY GMUNDARY CONDETTUNS In PLASTIC MATRTX (-RIDN)

no 7 I $=1$, NRF

IF(IPC(I).FO.1) GO 107

DKP $J=1$, NPC

7 CONT INUE

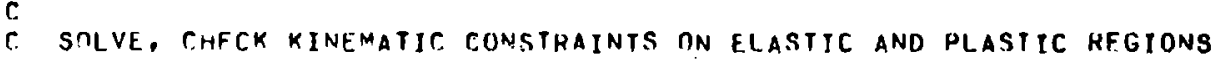

C AND ITERATE IF NECESSARY

CALL ITER CNFP,NNP, NRFP, TOSP,ISP,NECP, NPCP,NI PP,

WTL T, IRC,D, DFT, IPELTS, RI AM, A, PP, DU, NFWP, NNFWP, DIAGP, RMTNC.

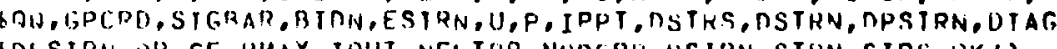

c.

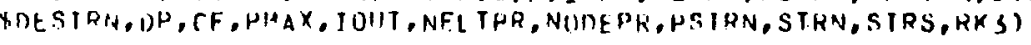

C ADHLY NFXT LUAD INCREMENT

(.) in 1 


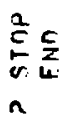

$\cong \bar{\simeq}$ 
COL \#EPCOT(1).DATA

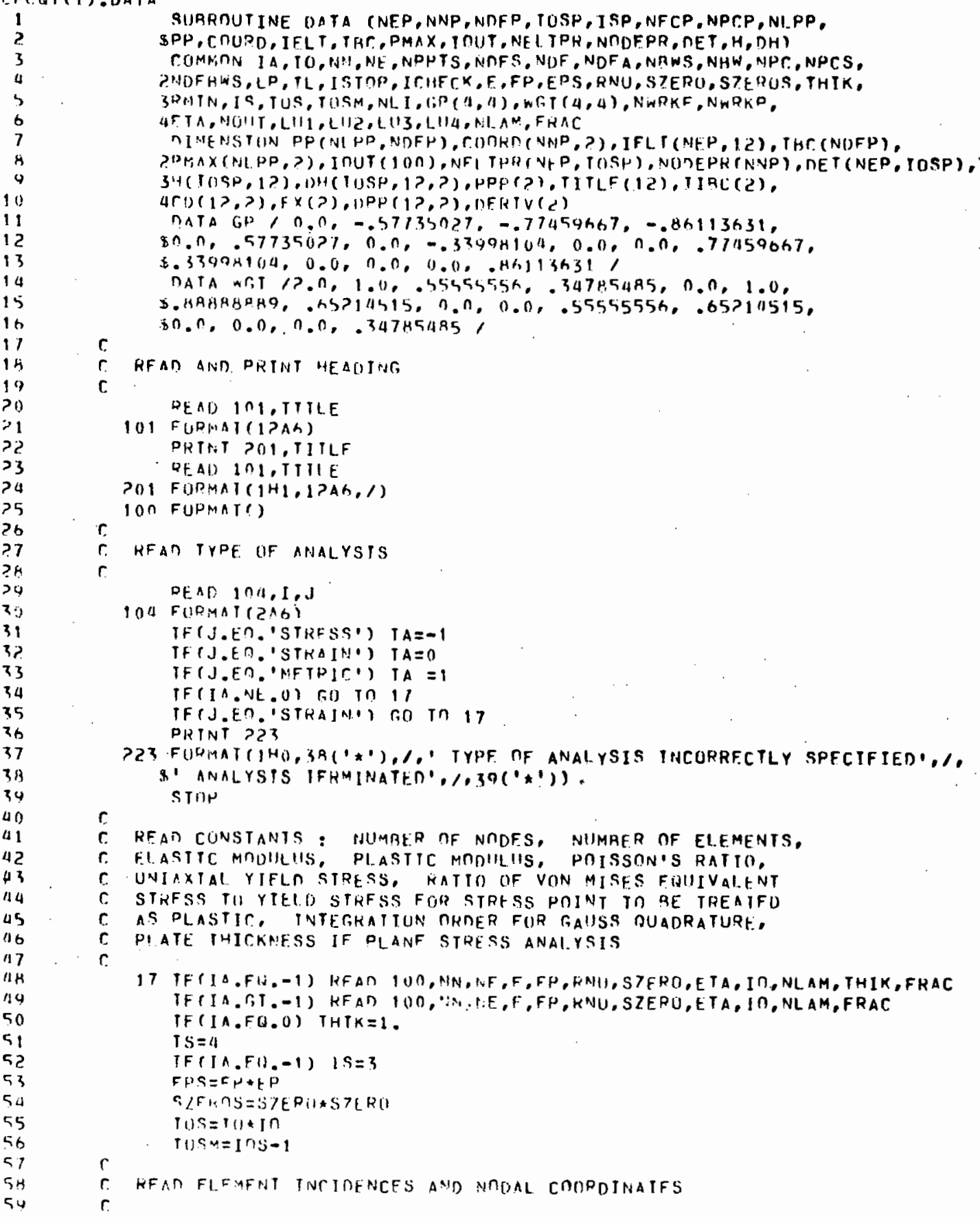


READ $1 \cap 0,((\operatorname{Cn}(1, J), J=1, ?), T=1,12)$

no $10.1=1,12$

r.Unkn $(1,1)=\operatorname{con}(1,1)$

$10 \operatorname{runkn}(1,0)=\operatorname{con}(1,2)$

(ri) 1013

A DEAO $100,(\operatorname{CON}(T, J), J=1,2), T=1,4)$

$t=!$

$i=1+1$

$\operatorname{TF}(\mathrm{J}, \mathrm{EO}, \mathrm{S}) \mathrm{J}=1$

TI=IFLTITE, I

$. J=I F L T(T E, J)$

$t=1+1$

$L L=I F L T(I E, L)$

$1=1 .+1$

$L L L=1 F I T(I F, L)$

กII $45 \mathrm{k}=1$, ?

$\operatorname{ni}, M=(C n(1, k)-C n(T, K)) / 3$.

$\operatorname{con} k \operatorname{con}(I I, K)=\operatorname{con}(I, K)$

$\operatorname{ron} k n(J J, K)=\operatorname{Co}(J, K)$

ronisn $(1 L, k)=C D(1, K)+D U^{M}$

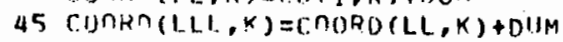

in rONITNIIE

$r$

r. DFIFHMINE CONSIANTS FOH SOLUTTON ROUTINE AND HALF BAND-WINTH OF ELASTIC MATRIX

$$
\begin{aligned}
& N B=0 \\
& \text { nI) } 1 ? T E=1, N F \\
& \text { ni) } 1 \text {, } 1=1,11 \\
& \text { I I }=I+1 \\
& \text { no) } i ? . t=11,1 \text { ? }
\end{aligned}
$$

MAX =AISS(TELT(IF, I) - IFLT(IE,J))

1?. TF(NAW,LT, AAX) NAW=MAX

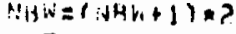

$N O F=N N * ?$

MI) $F A=N \cap F+$

Mi) $F S=N \cap F-$

NHS:NS =NAK-

A!UF I INSS $=N D F-N H W S$

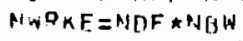

PIN MFADINIGS AND VALUES OF CONSTANTS

PLINTT ?1?

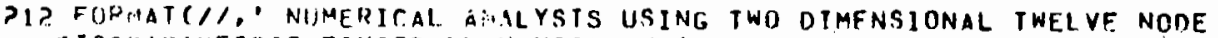

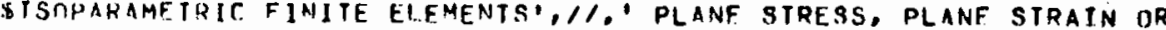
WAXISYMMETRTC PQOCRAM., //," CIIHTC INTFRPOLATION FIINCTIUNS', $/ 1$,

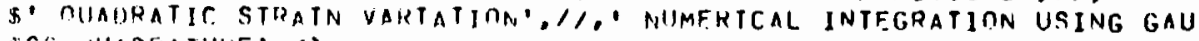
\$S Pis TiN 25,10

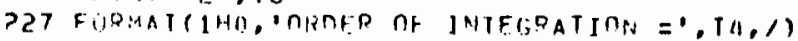

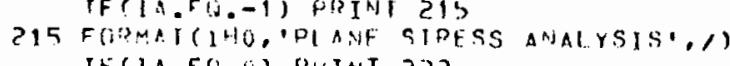

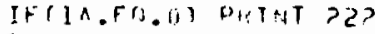

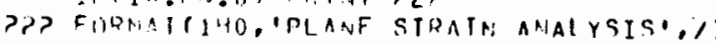


IF(IA FGA.1) PHTNT 203
203 FOPMAT(1HO. AXTSYMMETHIC ANALYSIS!, $)$

DRTHT ?:11

11 FORMAT(IHO, VION MISES YIFLO CONDITION?,1)

PI TFEEP. PJE.0.0) PKINT ? 14

IT (ED F

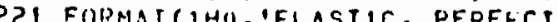

PIC:: ANALYSIS TERMINATEN WHFN DFiGT 347 , ETA

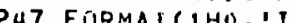

STHIN',FH.3, OF YIELD STRESS TRFATED AS PIASTIC, 13 PETHT >? TO, TIILE

?D FUQMAT $(1 H O, 1 ? A C, 1)$

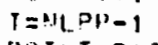

PQTIT P 13 , MINP, NEP, NIJFP, NFCP NPCP

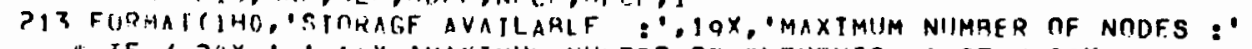

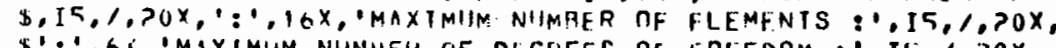

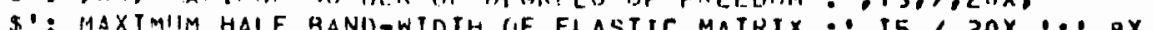

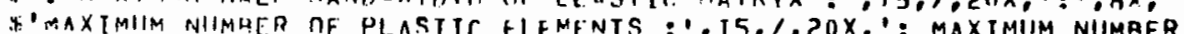

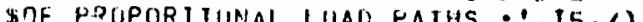

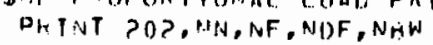

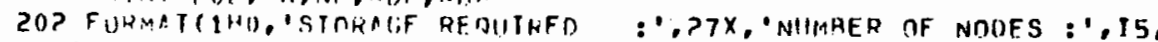

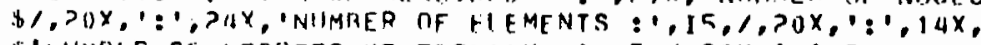

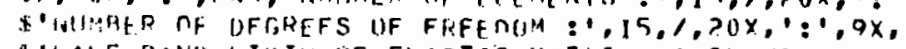

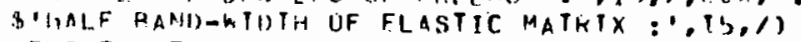

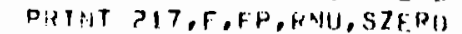

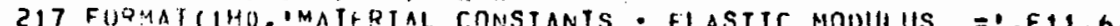

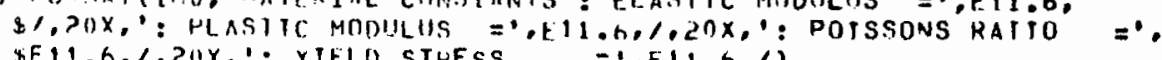
DF (IA Fí-1) PQINT 21 STHIK

21h FOPMAICIHO. PLLAF THICKNFSS

$=\cdot, 11, h, 1$

CHETKK AVATLAHLE STURARE

$: \cdot, F 11,6,11$

$$
\text { IF (NIRF.LF.NLFH) GO TO } 52
$$

31 FUPHAT(IHU,GO("1),1,1H, 'AVALLAPLF. STORAGE EXCEEDED: TON FEW RO

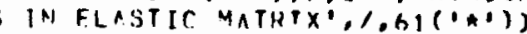

$\sinh$

S? IF (NAN.LF.NECP) 60 TO 46

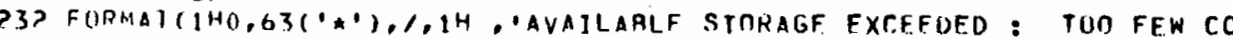
DLUMIVS IN ELASITC MATRIX',1,64('*'))

$$
\operatorname{sinP}
$$

46 TF(NN.IE.NAP) 60 In 47 OKTHT $>33$

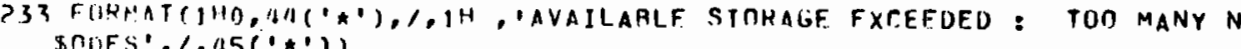
$\sin \sin ^{-10}$

47 IF (NF. IE. NFP) ROO In 138 DRTI:T P3A

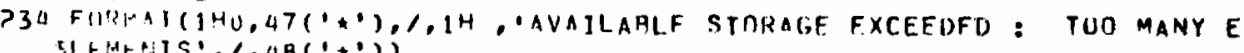

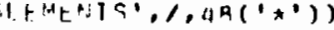

$$
\sin
$$

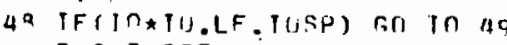
$\because \pi 14,>35$

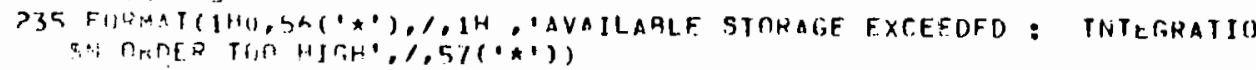


OAVAILAALF STMRAGE EXCEFDFD : TOO MANY R SIWS IN DEF ORMATION MATRIX', /.66('-1))

PRINT Noidal coupotwaies

SA TF(IA.1.T.1) DRTNT P04

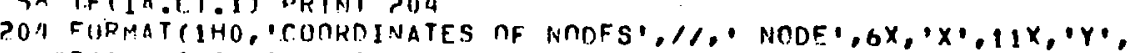
S3(10X, innof, $\left.\left., 6 x_{0}, x_{1}, 11 x_{0}, y^{\prime}\right)\right)$

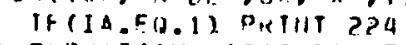

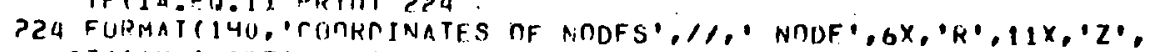

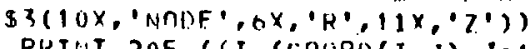

305 FORMAT(SOU(1H, I I, 2E $12,5,3(6,, 13,2 F 12,5), 1)$

r.

PRINT HLEMENT INCTIENTES

PRINT POA

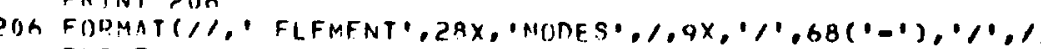
PEINT ?U7, ( (T, (IFLT $(i, J), J=1,1$ ? $), i, i=1, N E)$

$c$ (2)

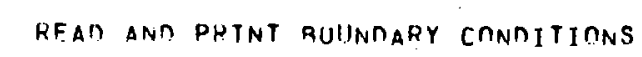

IH (IA.LT.1) PRTNT ?OR

POR FURHATCHHU, BOUMUARY CONDTTIONS: 0 - CUNSTRAINED',

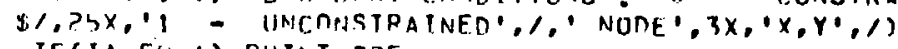

325 FURMAT(IHO

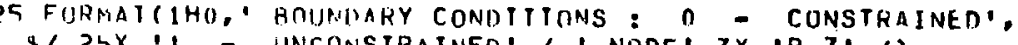

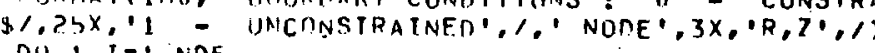
nU) $1 \quad 1=1$, N N F

1 THC. (T) $=1$

TEAD 100.1

IF (1.E. E.-1) r.ll in

READ(0,inO) $(I T H C(H), J=1$, ? PEAD 100,1

$k=1$

$k=k+1$

PEAD $(0,100)\left(I \cap U T(J), J=1, K_{1}\right)$ IF $(I \cap U T(k) . N F .-1)$ GO in 3 $k=k-1$

no a $I=1, K$

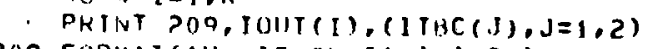
FORMAT $(1 \mathrm{H}, 13,1 \times, 11,1,1,11)$ $I I=(\operatorname{IoIJI}(I)-1) * 2$

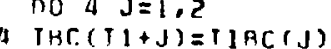

140.

c. RFAT ANA PRINT LUADTNG PROGRAMMF

$?$ PHTWT ?IO

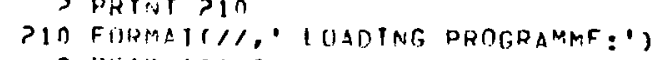

O REAB 1 InO.

$i_{k}=1 k+1$

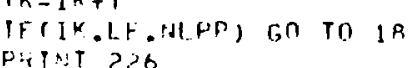


IFCI

IF 1 (ED,-1) G() in 5

READ(0,1no) TJ,I,J,Pmax(TJ,?)

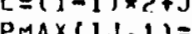

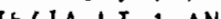

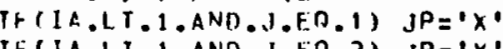

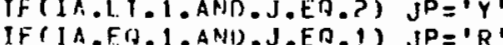

IF (IA.FB.

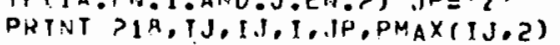

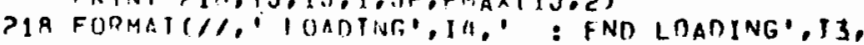

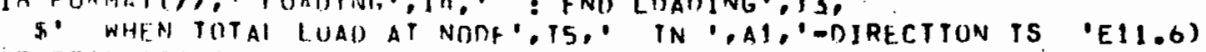

43 READ 1 ONO.

$$
\text { IF (J.LF.O) GO TO } 10
$$

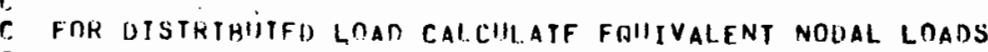

READ $(0,100)$ TE,ISIRE, $F \times(1)$, FX(2), ILD

$R=1$.

ro in $(23,24,23,24), 15 I D E$

23 IF(ISINE, ER, 3) $s=-1$.

TOFHIV $=1$

ni) $3 / 1$ i $x=1$, in

$R=\operatorname{rit}(10, i x)$

34 CALL. HMAT (IOSP,H,OH,IX,R,S)

r.U in 35

21) IFIISINE, ER.? $R=-1$.

Ti)FHi $R=5$

ni) 3 t $T Y=1$, in

$S=T P(I \cap, T Y)$

36 T.ALL HMAT (INSP, $H, \cap H, I Y, R, S$ )

35 nil a4 $1=1,12$

nu $44, \quad J=1$, ?

IF(IIU. P.F. I) $H A \cap=F X(?) / F X(1)$

no \&? $16=1$, In

no) $3 A \quad i=1$, ?

NERIV $(T)=0$.

$n_{0} 39 k=1, i 2$

39 $D E D I V(T)=D F R T V(I)+D H(I F, K, I D E R T V) * C O N R \cap(L, I)$

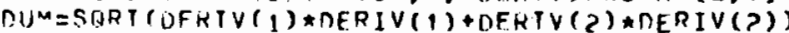
IF(IA.NE. D) GO In 40

THIK $=0$.

ro $41 \quad i=1,1$ ?

41 THTK $=T H I K+H(T G, T) *(., O N K,: i L, 1)$

THTK $=T H I K \star A . P B 31953$

Un $W T=W(, I(I N, T G) * T H T K * D 11 M$

IF (1I.1),EO.1) Gก TO 55

no $5 \mathrm{~h} \quad T=1$, ?

$F \times(1)=0$.

not $57, \quad j=1,18$

$k=T H \|(1 F, 1)$

S7 $F \times(1)=F \times(1)+H(t g, a) * \operatorname{contan}(x, 1)$

5h $F \times(1)=F \times(1) * R A n$

55 की 4 \& $1=1,12$ ? 


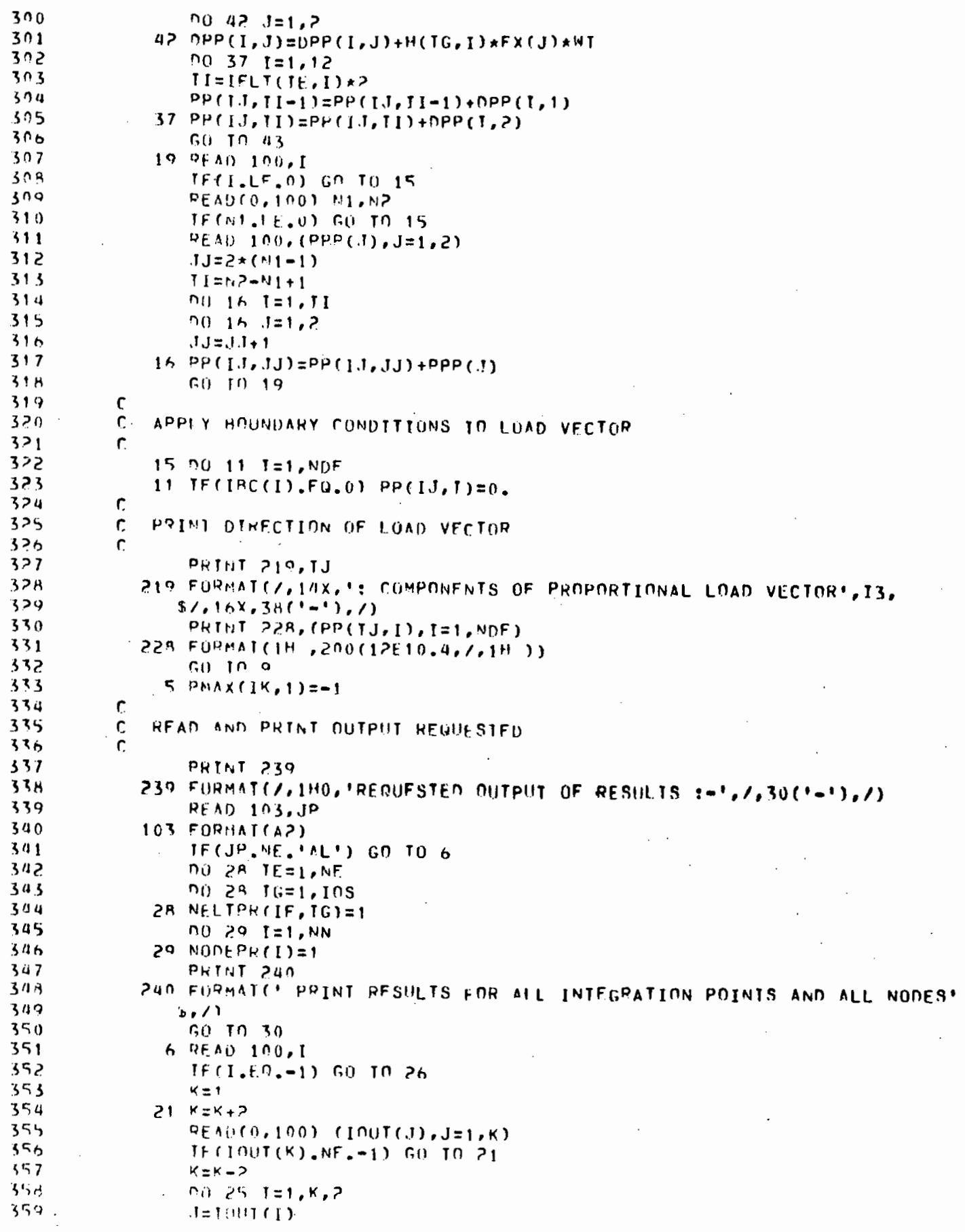


24 FIIRAAT(IH, OPHINT RESUITS FOQ :- (ELEMENT : INTEGRATION POINT)') PKTHT PQ?, (InUT $(J), J=1, k)$

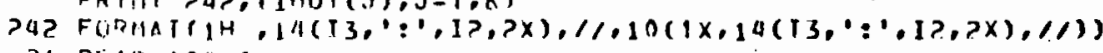
PEAD InO.I

F(1.E).-1) fon in 30

$31 \quad \begin{aligned} & x= \\ & k=x+1\end{aligned}$

QFAO $(0,(\cap 0) \cdot(\operatorname{InUT}(J), J=1, K)$

if $(1 \cap$ N $(k)$.NF. -1$)$ r,o in $x_{1}$

$k=x-$,

nu $27 \quad t=1, k$

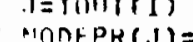

PQTNT 243

343 F(JPMAT(1HI), 'PRTNT RESULTS FOR NONES :-1)

DKINT ?aA, (IDUT(, $), J=j, K\}$

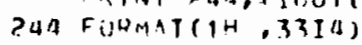

30 PEAU 100,1

3? $\begin{aligned} K & =1 \\ k & =k+1\end{aligned}$

$k=k+1$
Pf $A 1)(1,10 u)(I n U T(, J), J=1, k)$

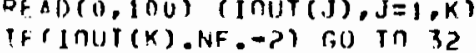

Pithit $>45$

?A5 FURMAT(IHO, PR SULTS RERUESTEN AFTER LOAD INCREMENTS :-9)

k=k-1,

It (InUi $(k), f n-11) k=k-1$

DKT:NT P44, $($ [NUT $(, 1), J=1, K)$

HET TIPI 
r.UL^FPCOI (1). HMAT

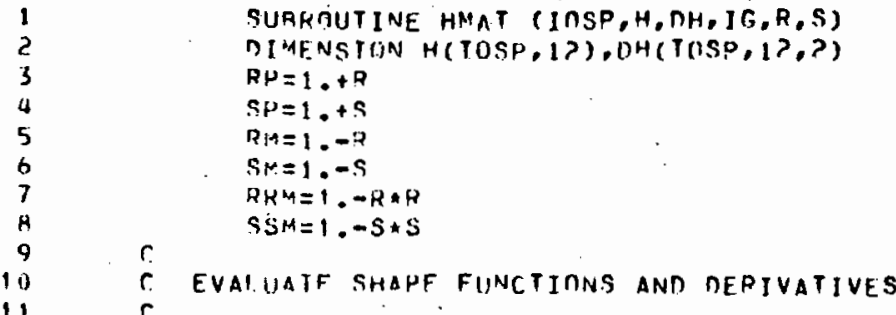

$c$

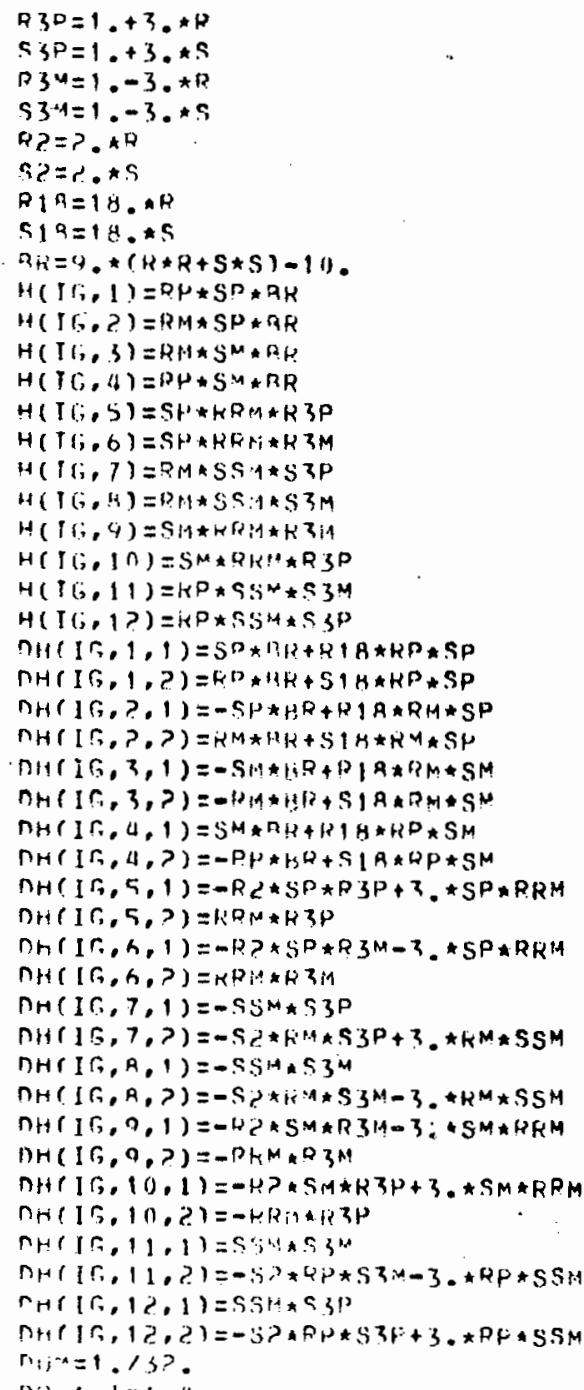




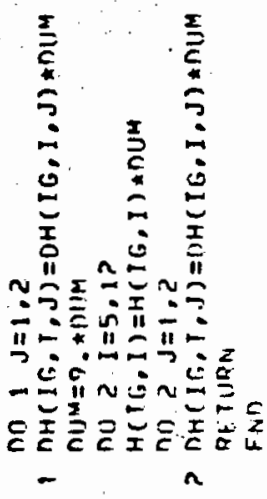

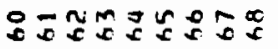




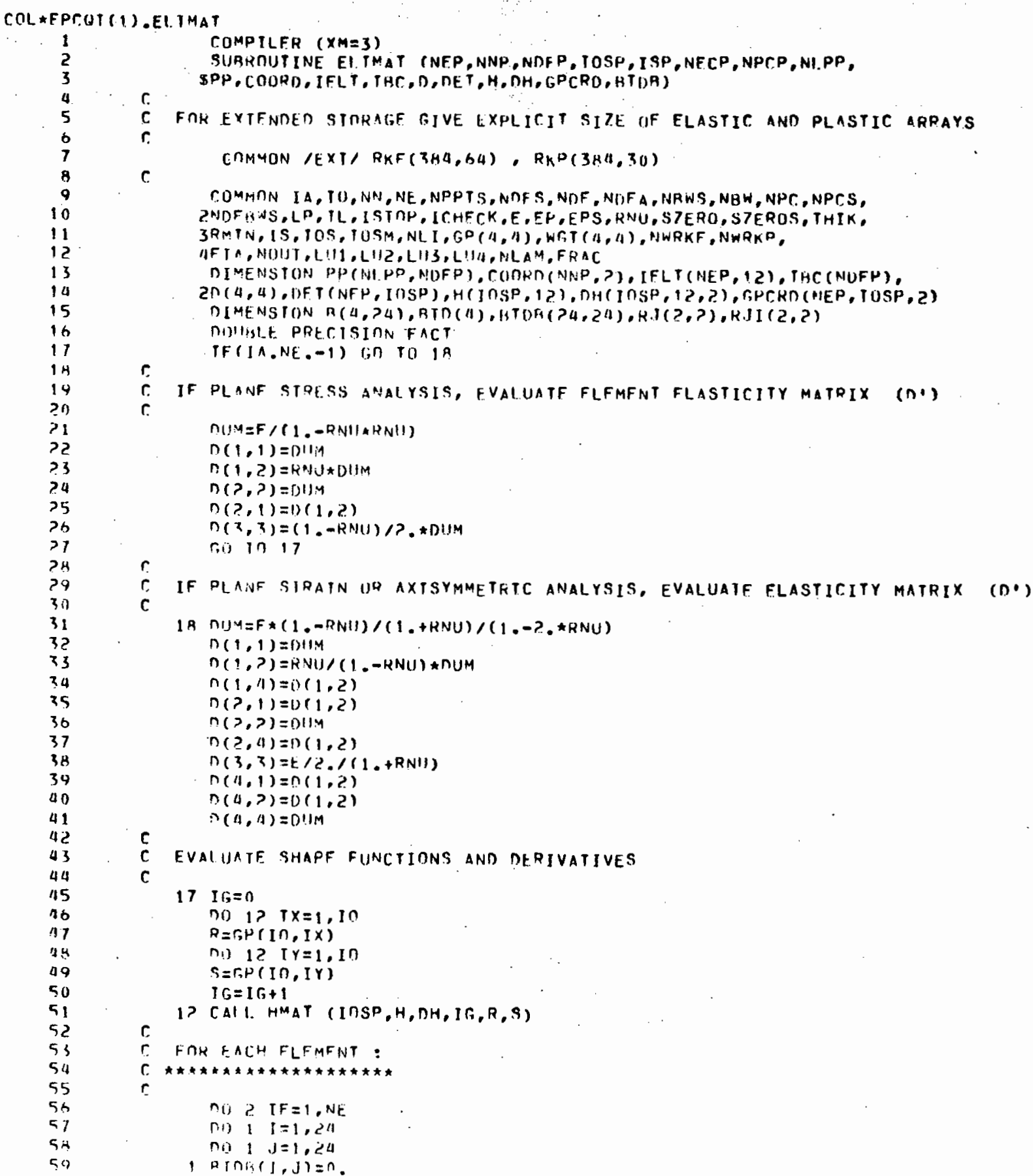




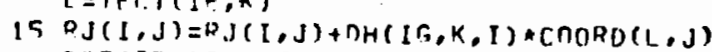

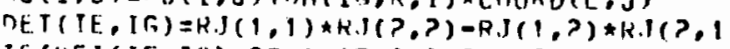
IF(I)FI(IF, IG). F, T,0.1F-n7) Go in 16

Jon Fol

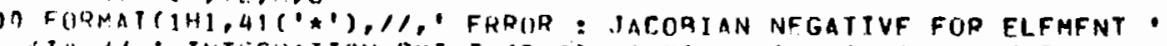

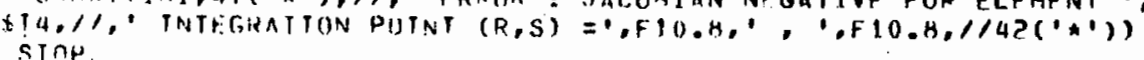

c.

EvaluatF inversf tif jactutan matrix

is กUM=1. MI)FT(IF, T(;) $P, J(1,1)=R . J(P, 7) \star 0, H H$ Q., $1(1,5)=-8 J(1,5) * 1) ! M$ 0 $Q_{K=-1} T(2, ?)=2.9(1,1) * 011 M$

$r$

EVALUATF STRATN-DISPLACEMENT Matrix (B')

no $2 n \quad T=1,12$

$k=k+?$
$L=K+1$

$x(1, x)=0$.

$n(1, x)=0$.

$n(?, K)=0$.

$n(?, i)=0$

Di) $21, i=1$,

$B(1, K)=H(1, K)+R J 1(1, J) \star D H(I[, I, J)$

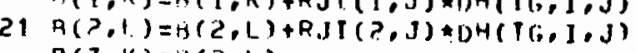

$R(3, K)=H(2, L)$

2n $P(3,1)=H(1, K)$

C CALCUI.ATE CAHTESIAN COURIINATFS OF GAUSS INTEGRATTON POTNTS

RHCRD(IE, I $1,, 1)=0$

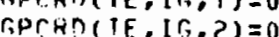

no $2 ? \quad r=1 ; 12$

$11=1 F L I T F, 11$

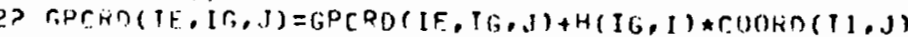




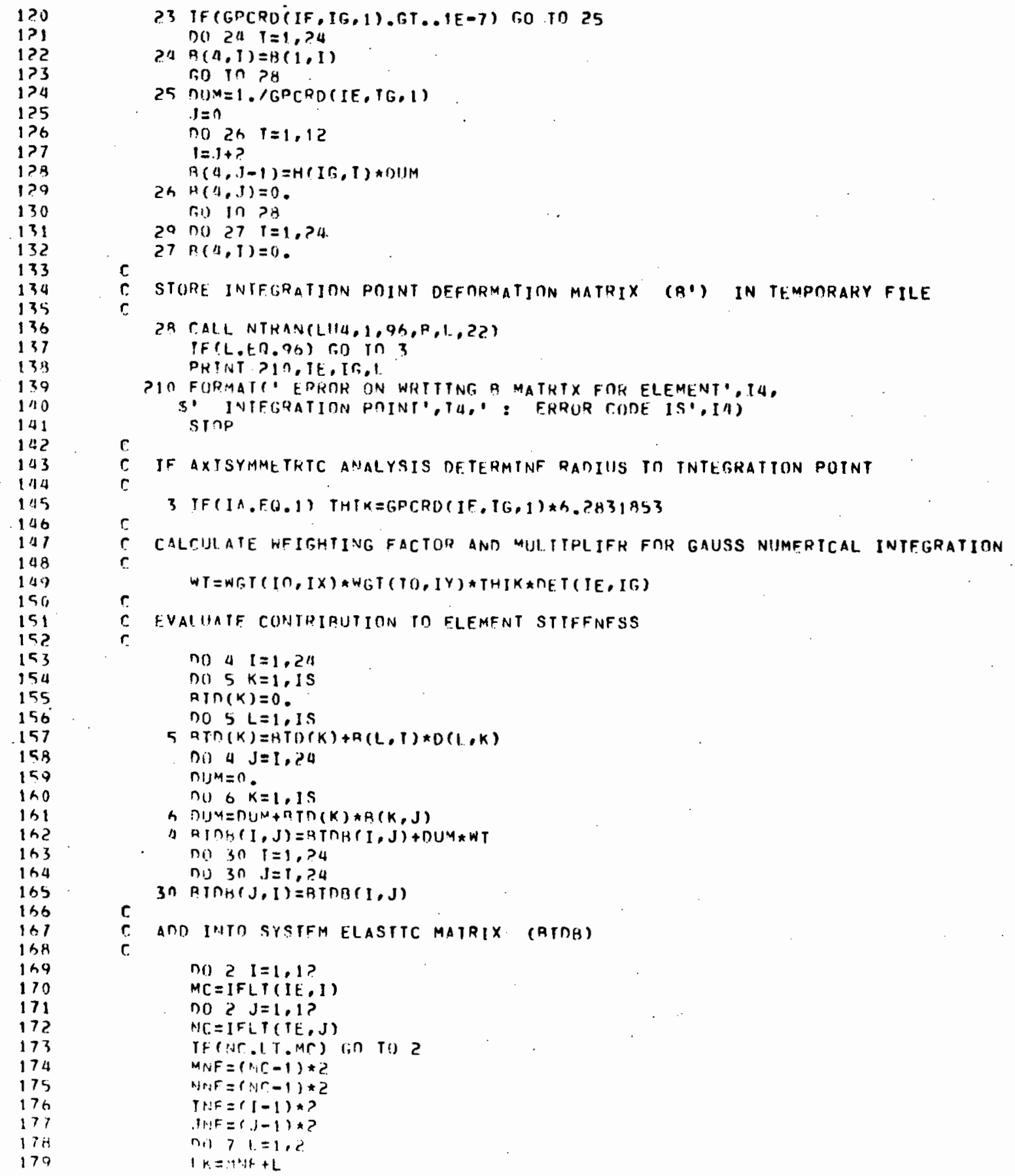




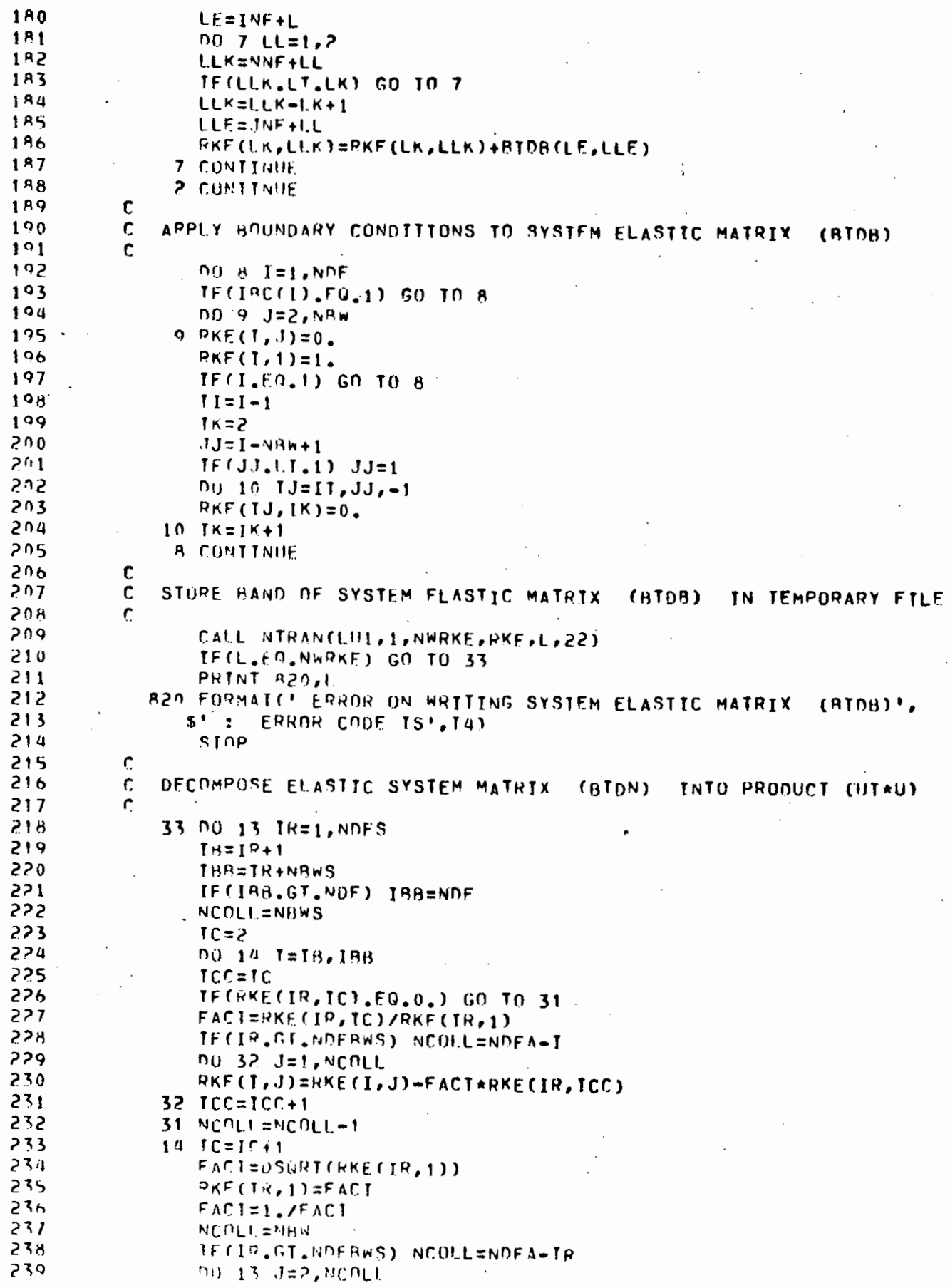




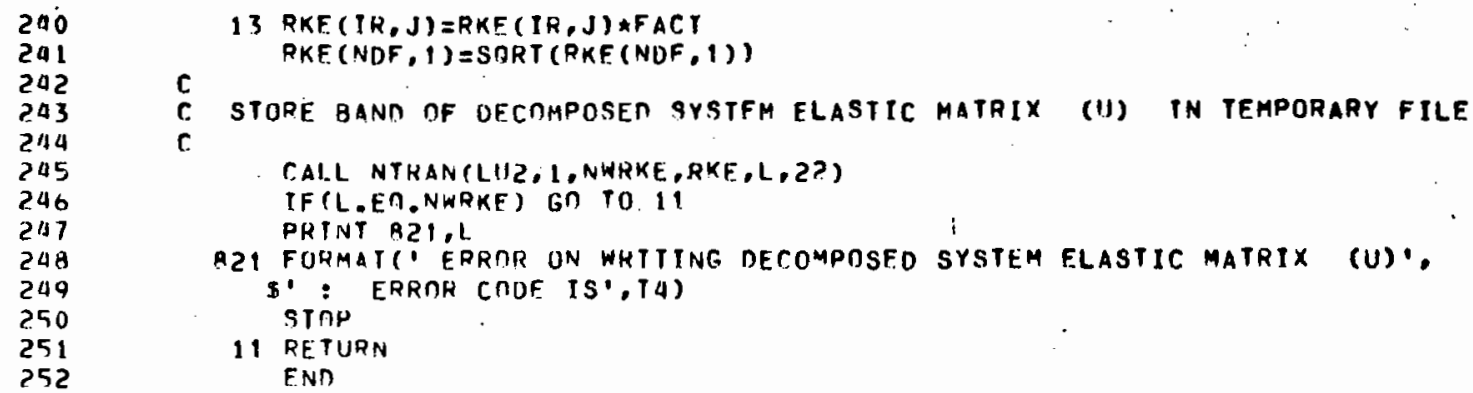


COL $\star E P C O I(1)$.SNLVE

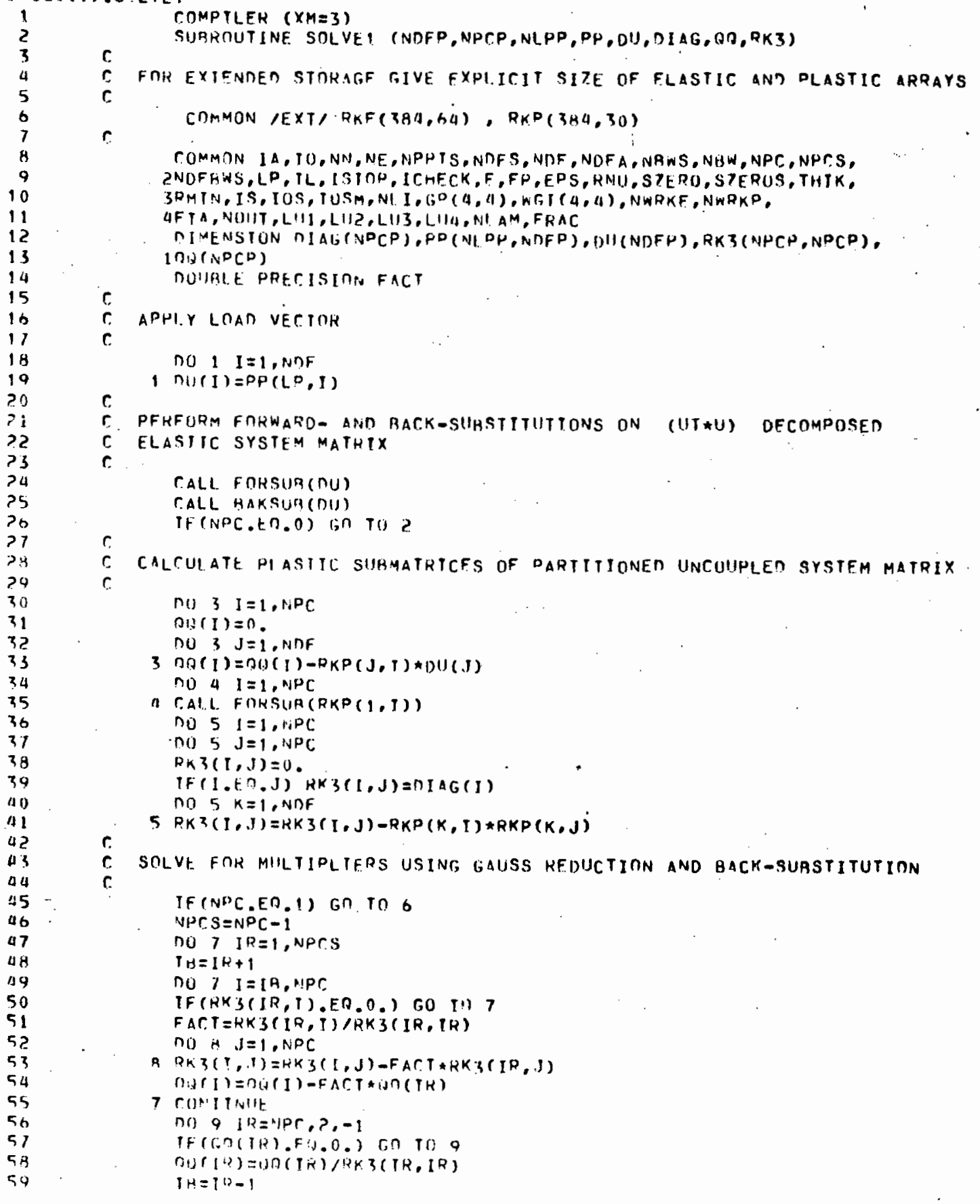


(1)/RK $3(1,1)$

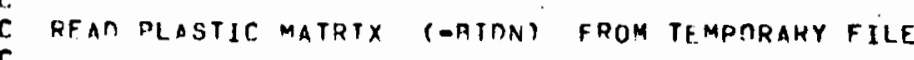

CALL NTRANCL!13,10,?,NHRAP,RKP,L, ?2) :

TF (L.EP. NWRKP) GO TO 11

R40 FORMATC, ER ': ERRIK (OOIF IS', T4)

r.

CALTUIATE HHS VECTUR MF PARTITIONFD UNCOUPLED SYSTEM MATKIX

II nO 1? $1=1, N D F$

nU(I) $=P P(L P, T)$

no 1 , $J=1, n e^{2}$

1) $\operatorname{nU}(1)=\operatorname{RU}(1)-\operatorname{RKP}(1,). * \lg ($.

$c$

SOLVE FOR UISPLACFMFNTS USINT FORWARD- ANO PACK-SUBSITTUTTON

ON (IITAU) DFCRMPOSED FLASTIR SYSTFM MAIRIX

CALL FIKSUA(DU)

TAILL GAKSUAG(DU)

C. SUHPOUIINE FOR FURMARD-SURSTITUTION ON (UTHU) DFCOMPUSEO FLASTIS SYSTEM MATRIX

SURROUTINE FORSUR (RHS)

TIME" "SION RHS (NDFP)

ni) $11^{D}=1$, NOFS

IT (RHSTIP).EO,O)

PHS $(T K)=R H S(T R) / P K F(I R, 1)$

$I B=I S+1$

$T H R=T K+N R W . S$

IF (IBT.GT.NOF) IRH =NDF

$\mathrm{I}=$ ?

DO 2 I $I=1 \mathrm{~A}, \mathrm{IBH}$

RHS (I) $=R H S(I)-R K F(I R, J) \star R H S(I R)$

?. $I=1+1$

CONTINUEE

RHS (NOF) =RHS (NOF)/RKE (NOF, 1)

DF TURN

SUGRUUTINF FOR RATK-SUHSTITUIION ON (UT:II) DECOMPOSFD ELASTIC SYSTEM MATRIX

SURKOUTINE BAKSUR (RHS)

DIMFNSTON RHS (NOFP)

Ri) 1 IR=NDF,,-1

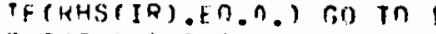

PHS(TK)=QHS(TK) PIKF TH,

$T H=\{B-1$

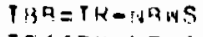

TF( IRH.LT.1) I $\quad$ B $(3=1$

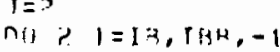

$\begin{aligned} & y=1+1\end{aligned}$ 


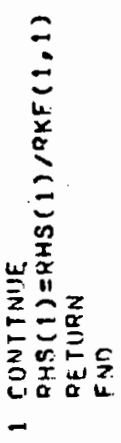

กำก 


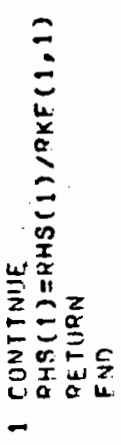

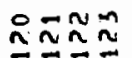




\section{COL *FPCOT(1).SOLVE?}

\section{COMMON /EXT/ RKE $(384,64)$, RKP(384,30)}

COMMON IA, IO, NN, NE, NPPTS, NDFS, NDF, NDFA, NAWS, NGW, NPC, NPCS, 2NDFAWS,LP, IL, ISTAP, IC,HFCK, E, EP,EPS, KNU, SZERO, SZEROS, THIK, 3RMIN, IS, TUS, TOCM, NL $1, G P(A, 4), W F, T(4,4)$, NWRKF, NWRKP,

CFA,NOHT,LLM,

(N) STINCLC)

AIJGMENT WITH LOAD VECTOR

DO IA $I=1$, NUF

C. STORE DIAGONAL PLASTIC MATRIX (NTON+OP)

Do 1? $I=1, \mathrm{NPC}$

$B O(I)=0$.

DO $1 ?, \quad I=1, N P C$

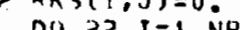

$\operatorname{Ros}(1,1)=010$

c

GAUSS RFDIICE TO UPPFR TRIANGULAR MATRTX

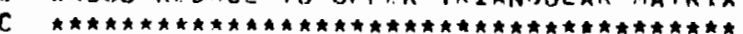

FIRST N RONS

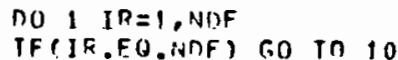

r. ELASTIC MUTRIX (RTOG)

$T H=I P+1$

$T H B=T K+N B W S$
$T H=T R+1$

IF (IRH.GT.NDF) IRH=NDF

NCOLI =NHWS

$\mathrm{IC}=2$

nu $B \quad 1=18,1 B$ B

IC $r:=\mathrm{TC}$

IF (KKE (IR,IC),FO.O.) GO TO 20

FAC. $T=K K E(I R, T C) / R K F(I R, 1)$

PF(IR.GT. NDFRWS) NCOLL =NDFA-I

DO \& J $J=1$, NCULL

$\operatorname{RKF}(I, J)=H K F(I, J)-F A C T$ KKKE $(I R, I C C)$

Q $T(: C=T C r+1$

no in $J=1$, NHE

It $\operatorname{RKD}(1,1)=R K P(I, J)-F A C T \star R K P([R, 3)$

n!U(I) $=$ nU(I) $-F A C . T * U H(T K)$

on NCOLI =NCOLLI -1

a $T=l r+$ 


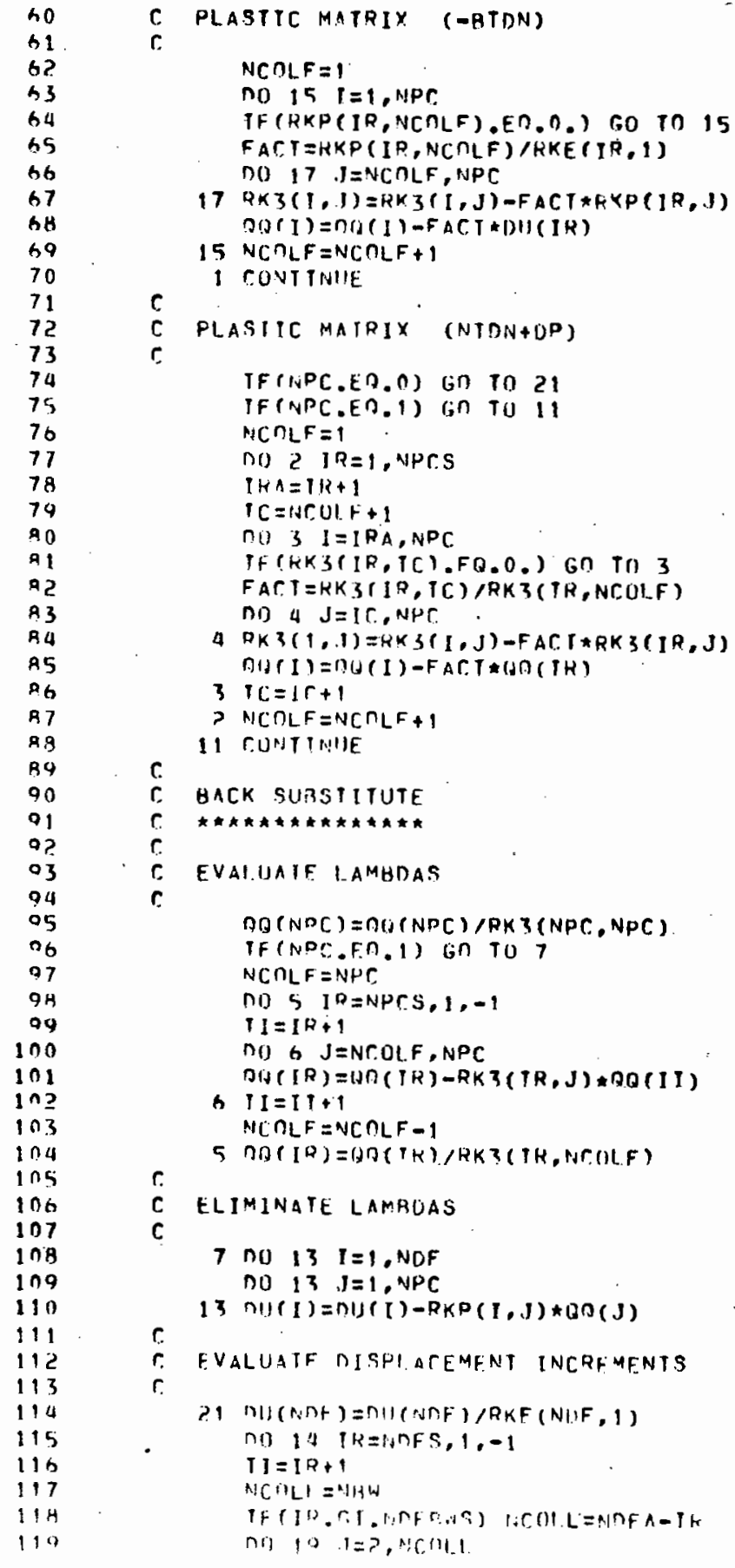




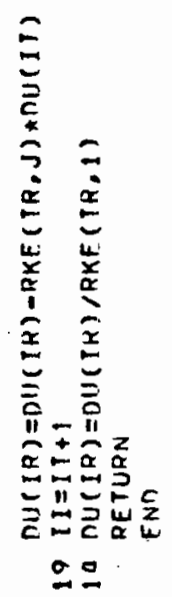

กㅗำำ 


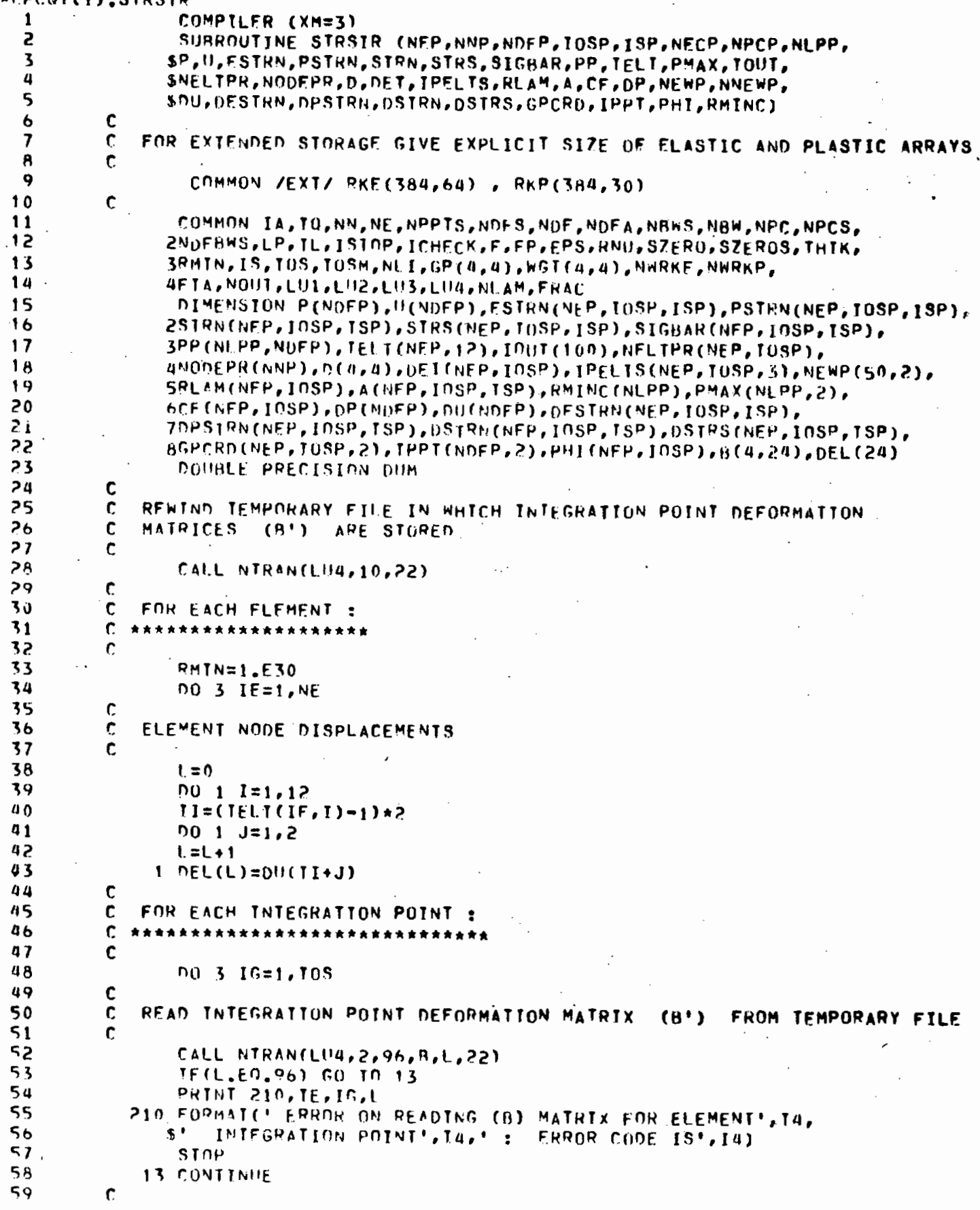




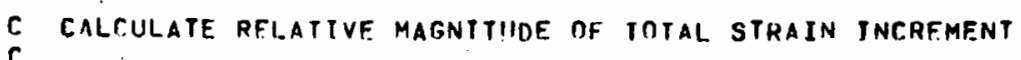

35 nO 2 I $=1$, IS

DSTRN (IE, IF, I) $=0$

ก) $2 \mathrm{~J}=1,24$

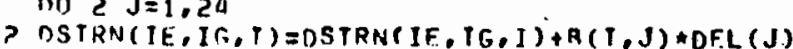

IF(IPELIS(IE, I $F, 1), E \cap .1)$ Gn TO

c.

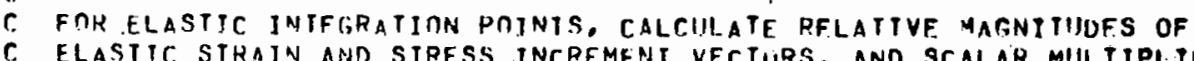

C ELASTIC STHAIN AND STRESS INCREMENT VECTURS, AND SCALAR MILLTIPLTER TO

CAUSE STRFSS PUTNT TO REAC.H YTELD SURFACF. FROM AL.I. FLASTIC

C INIFGRATION POINTS NETERMINF SMALLESI SCALAR MULIIPLIFR

ni) 4 I $=1$, IS

กPSIRN IE, TG, I ) = O

HESTRN $(1 F, T G, T)=N S T R N(T E, I G, I)$

NSTRS $\left(T E, I T_{0}, T\right)=0$.

no $4 \mathrm{~J}=1,1, \mathrm{~s}$

a NSTRS (TE,IF, I)=DSTRS $(I F, I G, I)+n(T, J) * D S T R N(I F, T G, d)$

TFACT $=1$

CAIL ROUT(DUM,DSTKS'(1E,IF, 1),DSTRS IF, TG, 2),DSTRS (IE, If, 3),

SISTRS (TE, If, 4), STGRAR(TF, If, 1), SIGAAR(TE, IG,?), STGAAR (TE, If, 3),

SSIFHAK(IF, (T, , 4$)$ )

$\triangle D=0+14$

IF (KMIN.GT.DN) KMIN=DO

c.

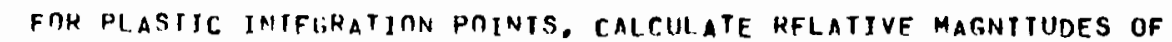

C EIASTIC STRAIN, HLASTIC STISAJN ANN STRESS INCREMENT VFCTORS

5 no $6 \quad l=1$, IS

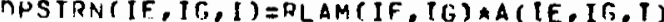

6 TE STPNI (IF,TG,I $)=D S T R N(T E, I G, T)-D P S T R N(T F, I G, I)$

ni) $7=1,1 \mathrm{~s}$

NSTRS $(T E, J(G, i)=0$

ni) $7 \mathrm{~J}=1, \mathrm{IS}$

7 OSTRS (TE,IR,I) =USIRS $(I E, I G, I)+n(I, .1) * 0 F S T R N(T E, I R, J)$

c.

C. CHETK IF PROPOSFD MAGNITUNE OF TOTAL LOAD IN CURRENT LOAD DIHECTION

C COMPLIES WITH LDADING. PROSFAMME

$I R=I F(X(P \operatorname{MAX}(L P, 1))$

nU $4=$ (PMAX (LP, 2$)-P(T K)) / P P(L P, I R)$

IF (KMIN.LT.DUM) GO TO 22

RiATN=DH/M

ICHECK =

c.

RINTC $(L P)=R M T N C$ (LP) +RMIN

MIJIIPLY ALL INCRFMFNT DUANTITIES BY SMALLEST MULTIPLIER, AND DETERMINE

CIBRENT TOIALS OF LOAD, DTSPLACFMENT, ELASTTC STRAIN, PLASTTC STRAIN

AND STRESS

nO $23 \quad 1=1$, NDF

$\operatorname{nin}(1)=R M T N * B H(T)$

$I t(T)=\operatorname{lil}(I)+n U(I)$

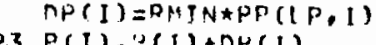

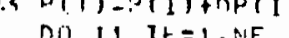

ni) 11 it $=1, \mathrm{NF}$

no $11 \mathrm{I}(s=1,10)$ 
STRN $(I F, I G, J)=S T R N(I F, T G, J)+D S T R N(I E, I G, J)$

FSTHA $(T E, I G, J)=E \operatorname{STRH}(I F, I G, J)+$ DESTRN $(I E, T G, J)$

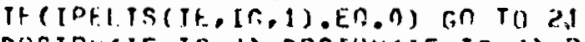

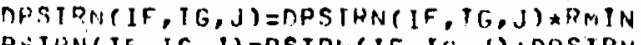

PSTRN $(T E, I G, J)=P$ STRM $(I F$, I $(,, J)+$ TPSTRN $(I E, I G, J)$

21 STRS(IF,TG,J)=SIRS(IF,TG,J)+กSTKS (TE,IG,J)

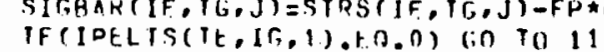

PL.AM(IF, IG $=$ RL AM(IF,IG) ARMIN

C. IN FLASTIC-PERFFCTLY PLASTIC CASE. NEHITAL-I UAOTNG SIRESS POINTS

. MOVF TANLIFNTIAL TO YIFLD SIIRFACF. THEREFURE. CORREC, CURRENT

STRFSSES RY hEIURNING STRFSS POINT TO YTELI) SUJRFACE

\section{IF $\triangle C T=$ ?}

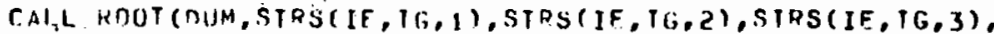

SSTPSIIF, IG,4), PSTKN(TE, IG, 1 , PSTRN(IF, TG, 2), PSTRN(TE, IG, 3), \$PSTRN(TE,IS, I ) )

C.F $(I F, T G)=$ NUM

STRS (IF, IG,J) $=S T R S(I E, T G, J) * C F(I F, I G)$

2h SISBAK IF, IG,J $=S T R S(I F, I(;, J)-F P \star P S T R N(I F, I G, J)$

c.

11 CONTTMUI

C. DETFRMINE CURDENT PLASTIC INIFGRATION POINTS. FOR FLASTIC IMTERTATION PIINIS C,HFCK PATIO OF VON MISFS EOUIVALFNT STRESS TO UNIAXIAL YTELD STKFSS GUR SIPESS POINT TO RE. TREATFU AS PLASTIC

\section{NPS $=0$ \\ L. I $F=n$}

A.PPIS $=$ ?

NASF ${ }^{N P} D=0$

NO) $25 \quad T E=1$, INF

no) 25 TG $=1$, Ins

IF (IPEI.TS(TF,IT, I) E.O.1) GO TO 3?

IFCIA, TEE.0) ROO IO 36

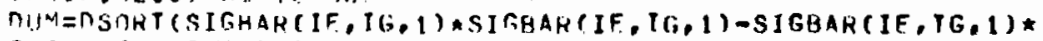

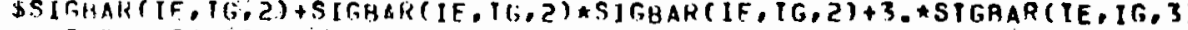
\$*STIGARP(TE, IG, 3))

r. 0 in 3 B

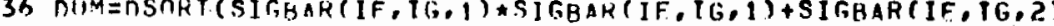

D*SIGRAR (IE, IF, ?) +STGAAR (TE, IF, I) *STGHAR (TE, IG, A) -STGAAR (TE, IG, 1$)$

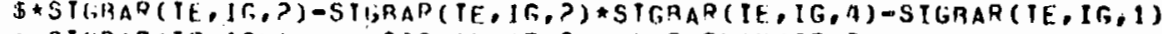

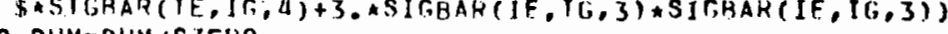

3A $\cap U Y=D U M / S Z E, K \cap$

IF CDUM.LT.ETAY:GO TO 25

TPFLTS $(I F, T G, 1)=1$

MINF WP $=$ M!NF WPT 1

ME. FP (NNE WP D T) $=1 E$

Sit $x$ (1)

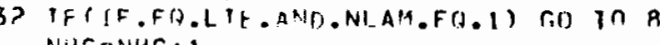

Mit $C=N H r+1$

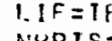

R:PDTC-RHPTS+1

IFDI (HOPTS, 1) $=T$

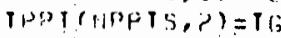




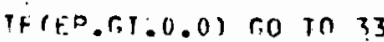

IF(RMIN/RMTNC (LP) GTT.FRAC) GO TO

NKNI $=N K N T+1$

IF (NKNT.1.1.3) FO IO 33

PETNT PQI, FHAC

201 FURMAIIIHU.83('*'),1,' LMAD TNCRFMFNTS LFSS THAN',FG.3,

3. IF TOTAL I UAS FOR PRECENING 3 LOAD INCKFMENTS'

S: ANALYSIS IFHMINATED', II,. RESUL TS AFTER CURRENT LUAD INCREMENT L istifP $=-1$

$10 ! 1 T(N \cap U T)=N L I+1$

c

$$
\text { a NKNT }=0
$$

DIIPUT. CURKEHT DUARTITIFS

33 CALL DUHTPUT CHFP, NAN, NRFP, TOSP, ISH, NF.CP, NPC.P, NLPP, WPSTRI, STPN, STKS, SIT,BAR, PMAX, IOUT, NFL TPD, NONEPR, SPHT, IPFLTS, RLAM, C,F ODP, OU, DFSTHN, OFSTRN, DSTRN, DSTRS, GPCRD, IPPT, Th, $1 \%, F$ STRN, NEWH, NNE WP, KMINC)

r. TF(ISTCIP EO, -1$)$ RO IO ?4

C. CHECK availagile STUPATEF

NHRKD $=N D F * N P C$ IF (NOC.LE.NPTP) GO TO 30 PhINT JU?

202 FURMAT(1HO,SH('*'),1,' AVATLABLE STURARE EXCEEDED: PLASTIC MATRI

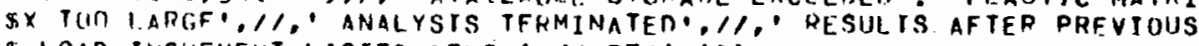
: LOAN INCKFMFNT I. ISTFO RFLOW'. $1 / .55\left({ }^{\prime} *^{\prime}\right)$ )

II) $(1 T(N \cap) T)=N 1$. I

NILI $=N L T-1$

CALL OISTPUT INFP, NNP, NDFP, TOSP, ISP, NFCP, NPCP,NLPP,

\$PSTRN, STRH,STRS, SIRBAR.PMAX, TOUT, NFLTPP, NUNEPR.

DPHT, TPFLTS,RI AM, CF, INP, IUU,DFSTKN, NPSTRN,DSTRN,DSTRS, GPCRD, JPPT,

\$P, U, ESTRN, NH WP, NNHE WP, KMINC)

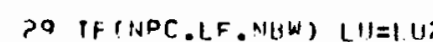

IF (NPC.GT.NHW) LUEI.U

CALL NTKAN(LII, 10, Z, NWRKE,RKF,L, Z?

IF (T.EO.NWLKF) GO TO 24

PRTNT RZOLL

20 FORMATC EPROA ON READTNG SYSTEM ELASTIC MATRIX (BTAB)', \$. : EPROR COUF IS', (4)

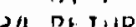

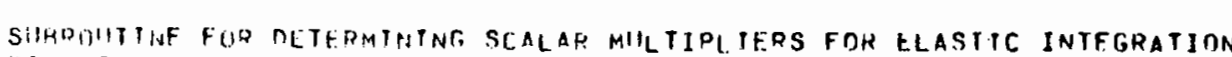
POINIS, AND FOR RF TUMNING STRFSS PUINTS IO YIELD SUAFACF FUR

PLASTIC INTFGRATIOTH POIHTS 


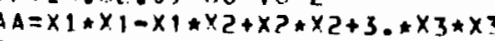

TFIIFACI.EN.? . ANN.EP.EN.n) (;n TO

$R Q=X 1 * Y 1-0.5 *(X X 1 * Y>+X Z * Y 1)+X 2 * Y Z+3 . * x 3 * Y$

$C C=Y 1 * Y 1-Y 1 * Y Z+Y Z * Y Z+3 * \star Y Z \star Y Y 3$

$A A=x 1 * x 1+x 2 * x 2+x 4 * x 4-x 1 * \times 2-\times 2 * \times 4-\times 1 * \times 4+3 * * \times 3 * x 3$

IFC[FACI.EO.?.AND. EP.En.0] Gn TO 1

$A H=X ! * Y 1+X P * Y Z+X 4 * Y 4-0.5 *(X 1 * Y Z+X Z * Y 1+X Z * Y a+X 4 * Y Z$

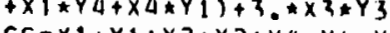

If $(I F A C$ T

PH:

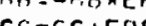

$P I=(D S P) K T(B B+B A-A A *(C C-S Z F P O S))-R B) / A A$

PETUTNA

PR = SZERO/OSURT(AA)

PETURiN 


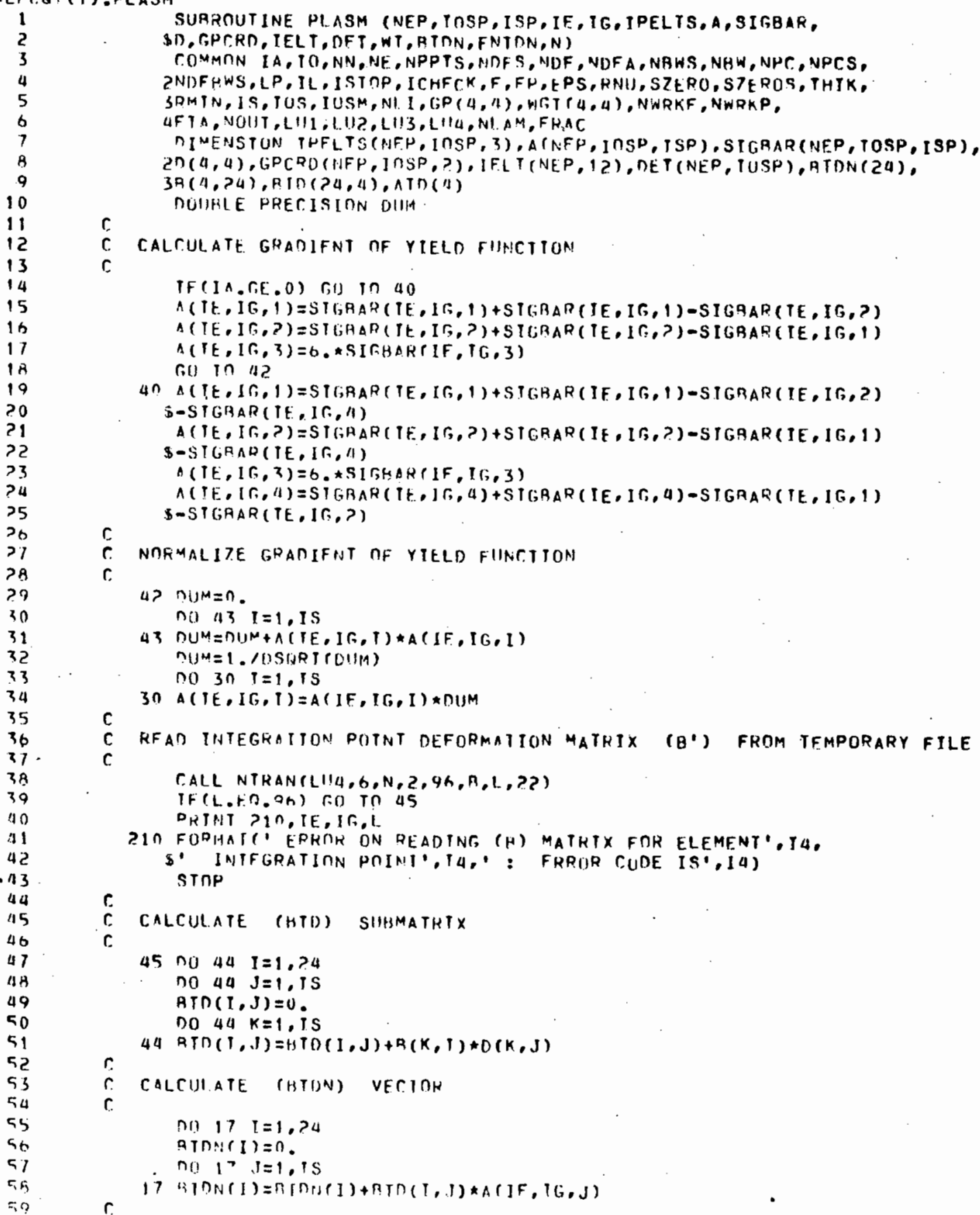


C CALCULATE (NTUN+DP) TFRM

no 1 is $J=1$, is

$\operatorname{ATn}(J)=0$.

no 1 \& $J=1$, is

1A $\triangle T D(I)=A T D(I)+A(T E, I G, J) * D(J, I)$

FNTDN $=F P$

no $10, I=1$, is

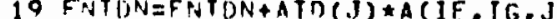

c. CALCULATE. WFIGHTING FACTOR AHD MULTIPLIER FOK GAUSS NIIMERICAL INTFGRATION

$I=(1(G-1) / I n$

$I x=I+1$

I $Y=1 r-10 * 1$

IF(IA.F(1.1) THIK=GPCPD(IF,JG.1)*6.2831853

$W T=W r, T(I D, I X) \star W G T(T U, I Y) * 1) F T(I E, I G) * T H I K$

RF TUNA 


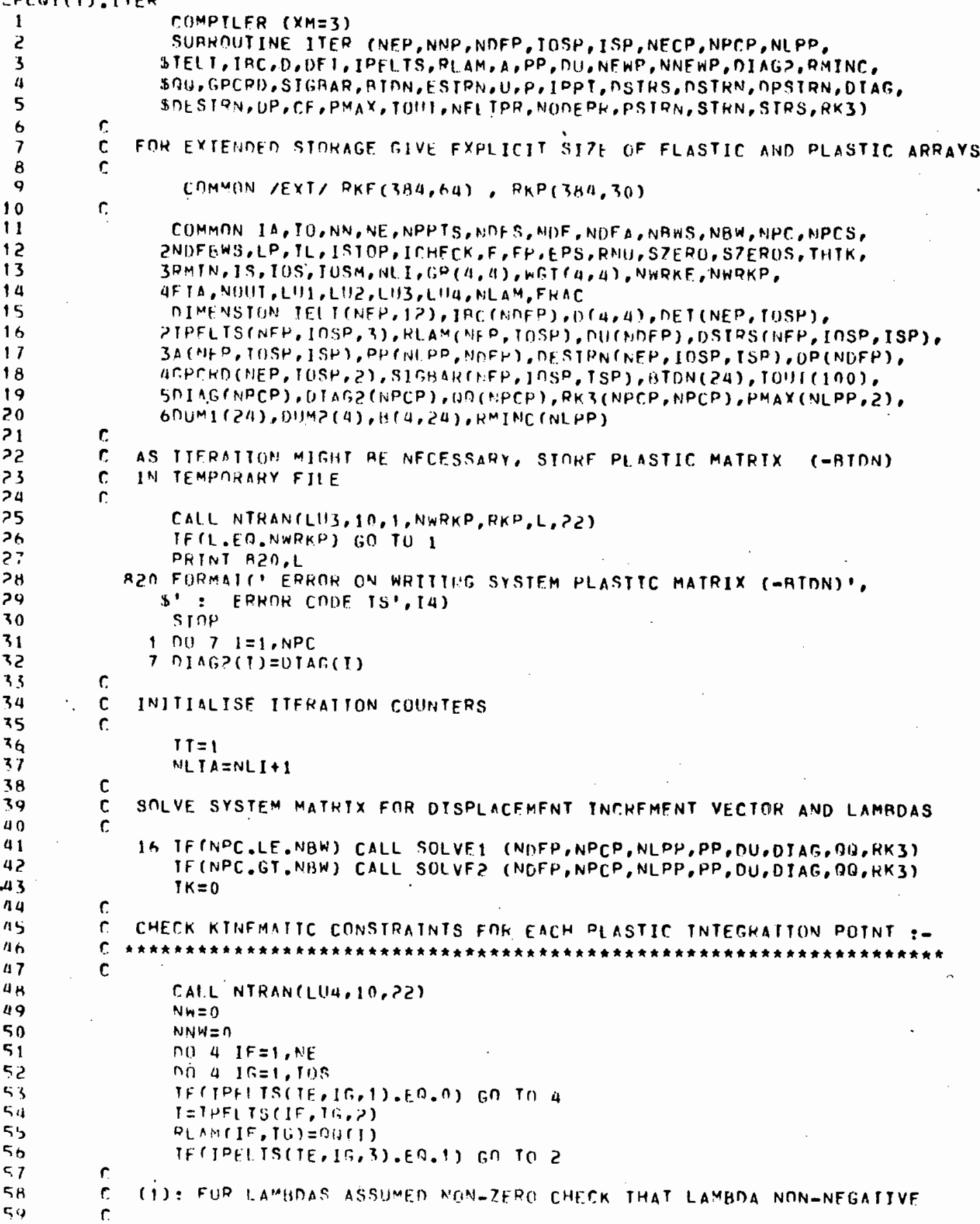




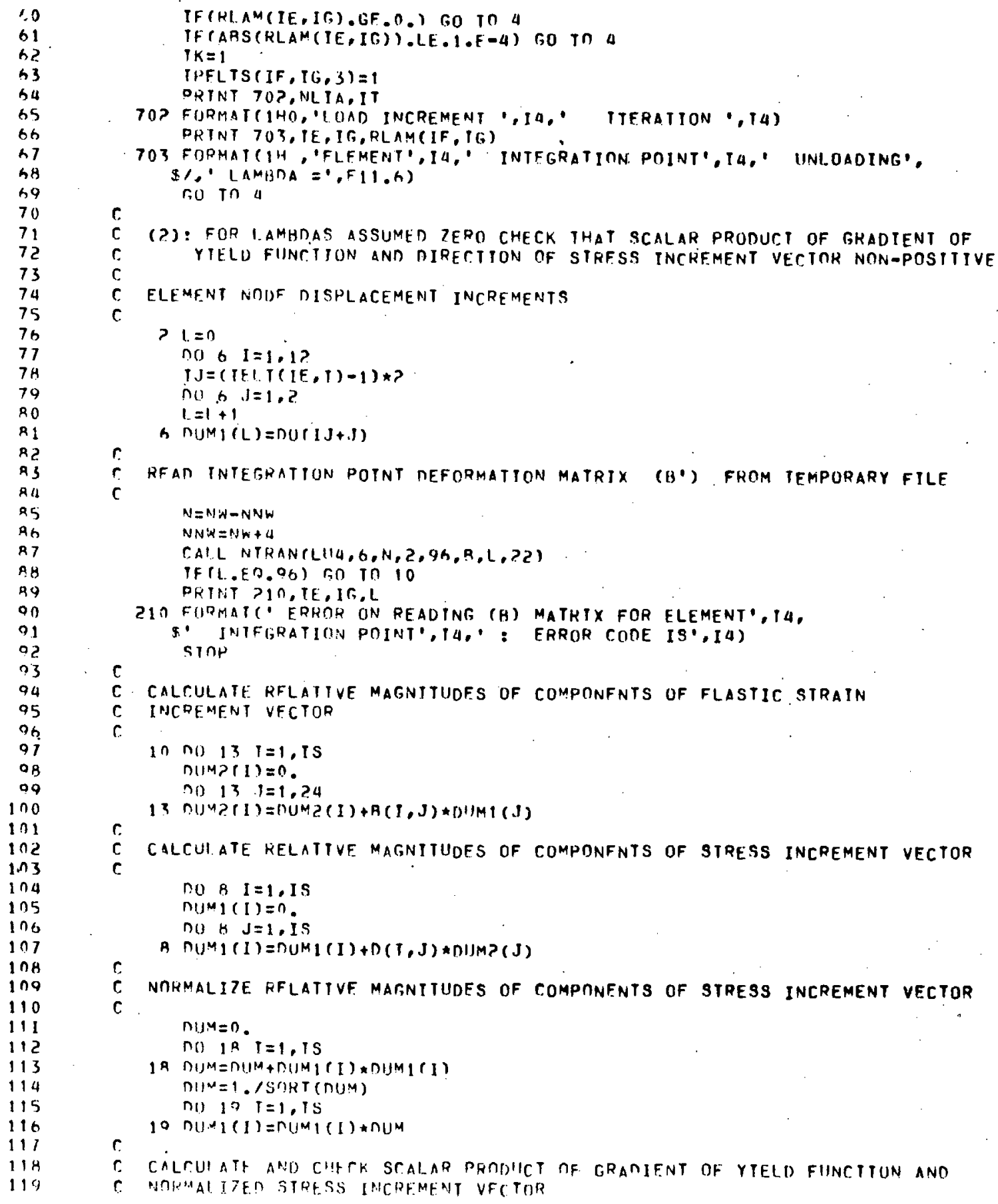


701 FORMATTIH, 'FLFMFNT', I4, INTFGRATION POINT', T4.' LOADING. $\$ \%$ SCALAK PRIINUCT OF GRADIENT OF YIELD FUNCTION ANO STRFSS INCREM WFT VERTIRE $=1, F(1, h)$

$4 \quad M: N=? N+4$

IF IJERATION CONSTRAINTS SATISFTED, CONTIHUF

IF $(1 \times . F(3.0)$ f.U in 12

r. IF ITERATION PRICFSS NUT CONVERGING TERMINATE ANALYSIS

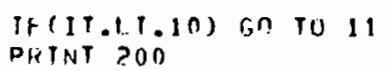

200 FORMAT $\left(1+10,5 \cap\left(^{\circ} *^{\circ}\right), 11^{\circ}\right.$ STILL. IN ITERATION LOOP EFTER 10 ITERATION

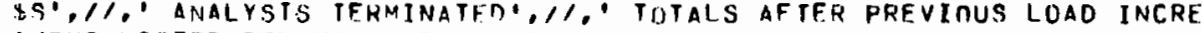
*MENT LISTED PELOW', $11,51(1 * 1)$
(i) In $>9$

C

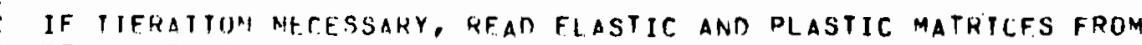

C TEMDUOARY FILFS

11 I $I=I T+1$

TFINRC.LF. NHW LUIIULU

TF (NPC.GT NNWW LHI=LUI

CALL NTRAN(LU1, 10,2 , NWRKE,RKF,L,2?

IF(L.E. NWAKF) GO TO 2 ,

DRTAT RBO,L

R3O FORMAIC EOROH ONA FEADING SYSTFM ELASTIC MATRIX ATRB., 5.: EPKOK CODF IS, TA

inte

CALL NTKANCLU1, I , ?, NWRKP,PKP,L,?2)

IF (L.EN.NWRKO) GN TO 17

ALO FOPMATC" ERHOR IIN READTNG SYSTEM PI.ASTIC MATRIX -BTON!, : ERROK CODF IS $\left.^{\circ}, \mathrm{T}_{4}\right)$

17 กU ?n $T=1, \mathrm{NPC}$

20 $D I A G(1)=n \perp A G ?(T)$

$r$

FOH PIASTIC INTFGRATION POINTS ASSUMED TO BE UNLOADING,

C. SIBGTRACT CONIRIRUTION IO PLASTIC. MATRICES (-BIDN) AND (NTDN+DP)

CAIL. NTRANILHA, In, PZ)

$N a=0$
$N$

ก) $1 /$ TE $=1$, IF

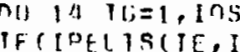

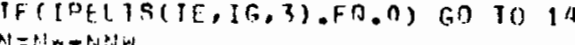

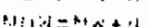

CALI PI ASH (EEP IOSP, ISP, IF, TG, IPELTS,A,SIGRAR, 
\$N, T,PCRN, IEL T, DFT,WT, ATON, ENTDN,N

NC I IPEL. IS (IE, IG, Z)

no $5 \quad I=1,1 ?$

NKR $=(I F L T(I E, 1)-1) \star 2$

NHF $=(I-1) \star$ ?

no $5 \quad J=1,2$

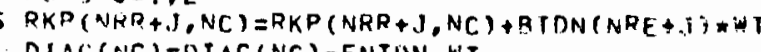

TIAC; $N C)=0 I A G(N C)-F N T Q V \times W$

TF(D)AR,(NC).GT.A.E-7) GO in 14

DO) IS $1=1$, NOF

nIA $;($ is $)=1:$

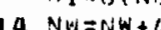

RE - SOL V

ก.) in 10

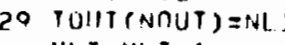

$N L I=N L T-1$

nu $27 \quad T E=1, N F$

nu 27 TG $=1$, InS

IPFLTS(IF, IG,
DO $27 \mathrm{~J}=1.1 \mathrm{~S}$

OESTRN $(I F, I G, J)=n$

7 NSTHST TE, $\left.1 C_{1}, J\right)=0$.

no ?a $T=1, N 1$.

Sa ${ }^{2}(1)(T)=0$.

CALL UHIDUT CNFP, NNP, NRFP, TOSP. ISH,NFCP.NPCP. NLPP,

TOSTKN, STRH, STKS.SIGRAR,PMAX, IOUIT, NFLTPR, NIJREPH,

SPHI, THFLTS, KI AM, CF DDP, DU, DF.STRN, OFS TRN, DSTRN, OSTRS, GPCRD, IPPT, SP, II, FSTRM, NE WP, NNI NP, RMINC)

r. ITEPATION PROC.EDURE HAS CONVERGFD:

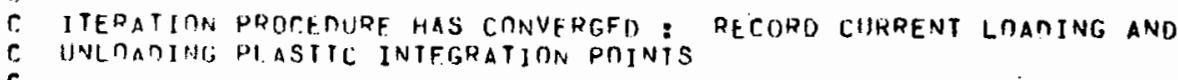

12 nO 3 IF $=1$, NE

ra 3 I $r=1$, Tus

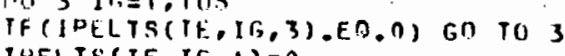

IPFL.TS $(I F, I G, 1)=0$

3 rUNITNIE

PE TUIR:

QE 
COLARPCQI(1).OUTPUT

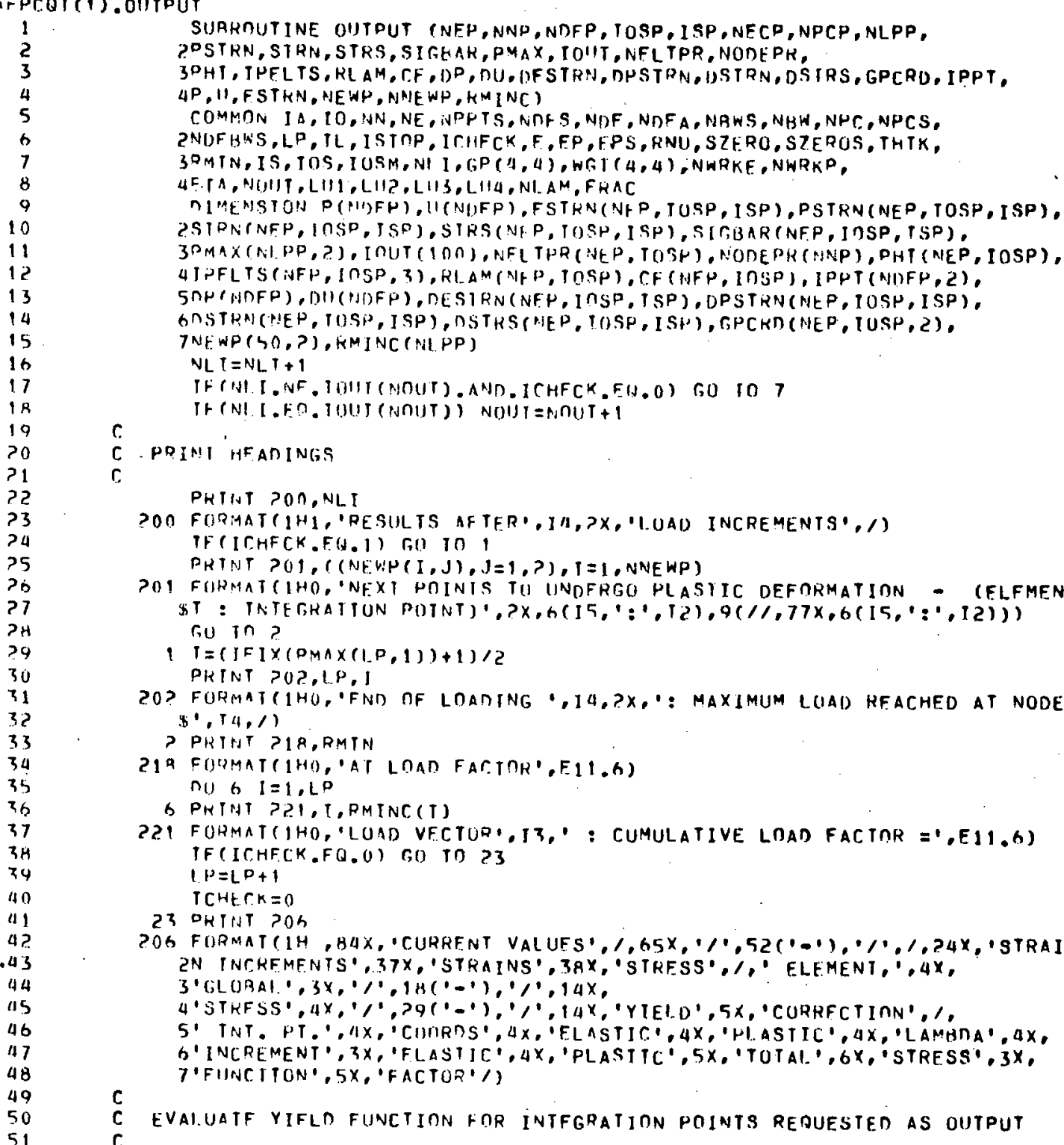

SUAROUTINE OUTDUT TNFP, NNP, NDFP, IOSP, ISP, NECP, NPCP, NLPP,

3PHI, IPFLTS, KLAM, CF ODP, DU,DFSTRN, DHSTPN, USTRN, DSIRS, GPCRD, IPPT,

4F. TA, N(UIIT, LIII,LL11?,LU13,L114,NIAM, FHAC

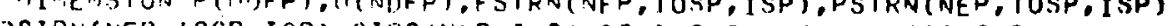

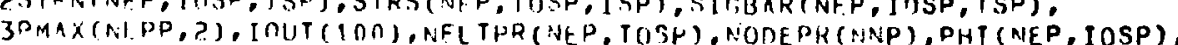

$5 \cap$ C

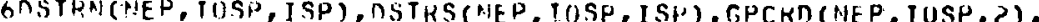

T)

PRIMI HFADINGS

FIIHMATCIHO, NFEX POINIS TO UNDFRGO PLASTIC DEFRRMATION - (ELFMEN

rou in

PKINT ?OS,LP,

19 FOMMAT

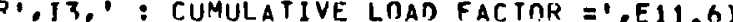

$1 P=L P+1$

$24 .$, STRA

2N INCKFMENTS', 37x,'STRAINS', 38x,' 'STRESS', ', ELFMENT, ', $4 x$,

4 'STRFSS, $4 x^{\prime}, 9$ O

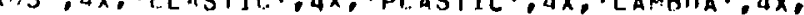

EVAIUATF YIFLD FUNCTION FOR INTFGRATION POINTS REDUESTED AS OUTPUT

$\begin{array}{lll}\text { nu } & 4 & I F=1, \text { AN } \\ \text { nol } & 4 & I \\ \text { I } & =1, \text { TUS }\end{array}$

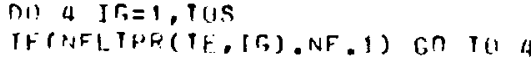

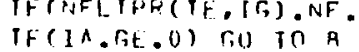

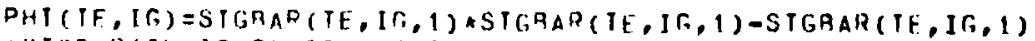

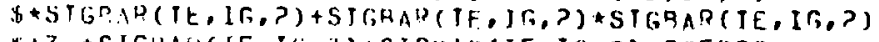

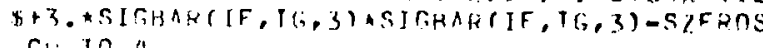


PRINT INTEgRATION POINT GHANTITIES FUR OUTPUI REGHESTFD

IF (1 A) $9,13,10$

9 nO $12 \quad I E=1$, NF

ni) $1 ?$ T $\mathrm{G}=1$, InS

IF (NFLTPR (IE, IS). NF, 1) GO TO I?

$c$

IF(IPELTS(TE, IG,i).tO. O) GO TO

PLASTIC INTEGHATtON POTNTS

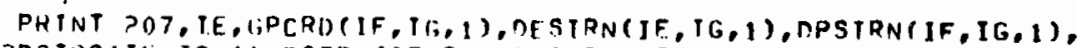
2NSTHS(TE, Ir, 1), ESTRN IF, IG., I), PSTRN IIE, IG, 1), STRN(TE, IG, 1$).$

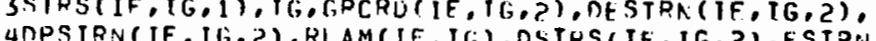

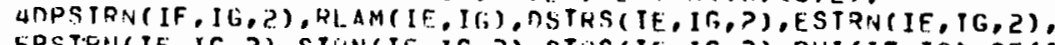

GOESTR:(IF, IG, 3 ) OPSTRN (IF, IG TFST

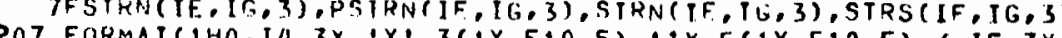

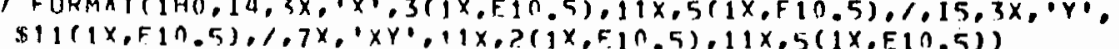
(10) 10 15

FLASTIC INTERIKAIION POINTS

3 PRTHT 205, IE, GPCRU(IF, 1G, 1), DESTRN(IF, TG, 1), DSTKS(IE, IG, 1) ZFSTHN(IE,IT, I), PSTRN (IF, TG, 1), SIRN(IF, IG,1), STRS(IF,TG,1),

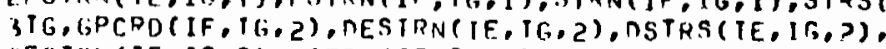

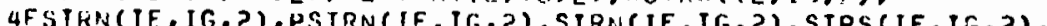
5PHI (IE, IF), DFSTKN(IE, IG 3), OSTRS (IF, IG, 3 ) FSIRV (IE, IG, 3 )

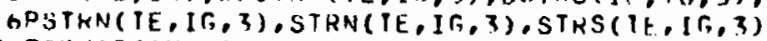

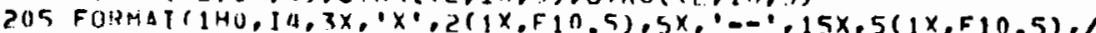

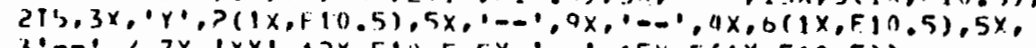

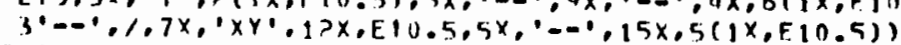

15 CONTINIIE

C. PLAME. STRAIN ANALYSTS:

13 กU ? 1 TE=1, NF

no 21 t $G=1,1 \mathrm{inS}$

TF(NFLTPR(TE, IG).NF,1) GO TO 21

IF (IPEL TS(IE, IO, 1)

PLASTIC TNTEGRATION POTNTS

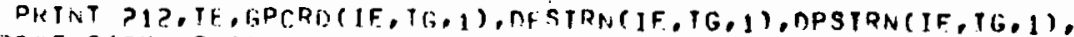

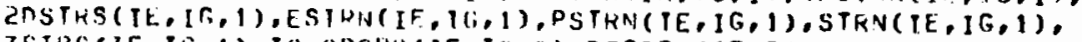

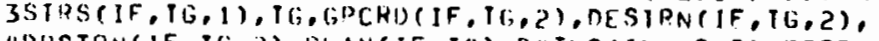

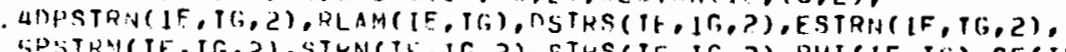

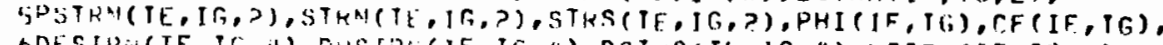

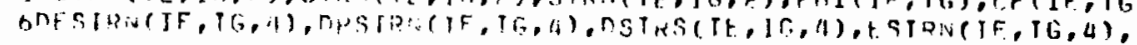


1)

?. FORMAT (1H0,11, 3x, $x^{2}, 3(1 x, F, 10,5), 11 x, 5(1 x, F 10,5), 1,15,3 x, 1 Y$, $211(1 x, F 10,5), 1,8 x, Z^{\prime}, 11 x, ?(1 x, E 10.5), 11 x, 5(1 x, E 10.5)$.

$31,7 x, 1 \times y, .11 x, ?(1 x, E 10.5), 11 x, 5(1 \times, E 10.5))$
Su in 21

: FLASTIC INTKGKATTON PUTNTS

14 PKINT 213, TE, GPCPOPIE, IG, 1), DESTRN(IE,TG,1), DSTKS(IE, IG,1), ZFSTRN(IE,IG,1),HSTPN (IF,IG,1), STRN IF,TG,1);STRS IF, IG, I),

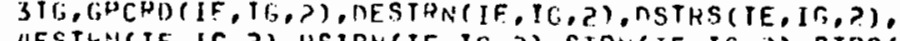

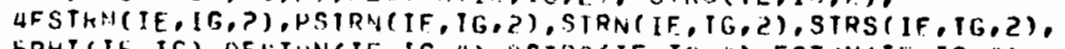
SPHI (IE, IG),DFSTHN (TE, [G,4), USTPS IIF, TG,4),FSTRN(IE, IG,4),

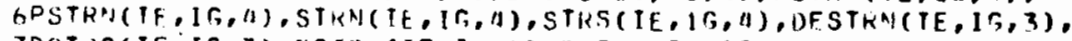
7NSTRS(TF, I,, 3$), E S I P N(I F, T G, 3), P S T R N(T E, I G, 3)$,

6STRNIIF,I $(6,3), 5 T$ TRS IF, T $T(;, 3)$

F(1R+A I (1H0, 14, 3x, $x^{\prime}, 2(1 x, F 10,5), 5 x, 1=-15 x, 5(1 x, F 10,5), 10$

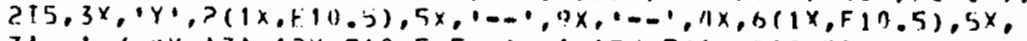
$3,-1,1, H Y, Z^{\prime}, 12 x, F 1 n, 5,5 x, 1=-1,15 x, 5(1 x, F 10,5)$,

$\left.41,7 x_{0} x^{\prime}, 12 x_{0}, 510,5,5 x_{0} \cdot-x^{\prime}, 15 x, 5\left(1 x_{0}+10,5\right)\right)$

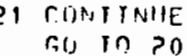

AXISYMMFIRIC ANALYSIS:

In nO ?? TE $=1, N F$

กO 27 TG $=1.1 \mathrm{NS}$

IF (NFLTPR(IF, IR).NF.1) GN TO 2 ?

SRINT $211,1 E, G P C R D(I F, T G, 1)$, DH STRN (IE,IG,1), DPSTRN (1E,IG,1),

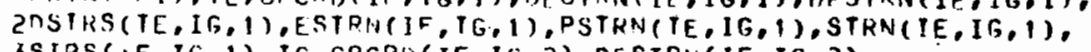

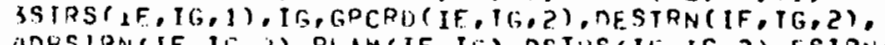

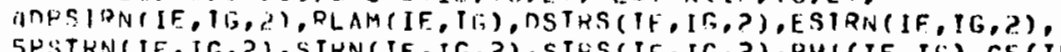

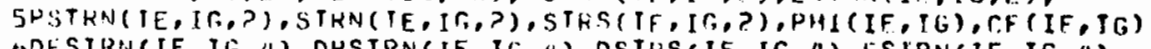

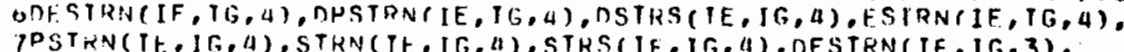

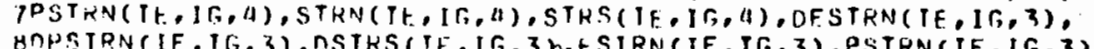

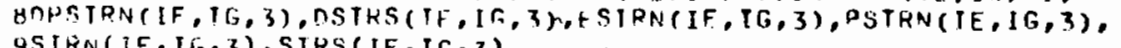

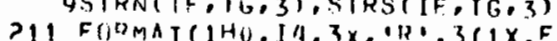

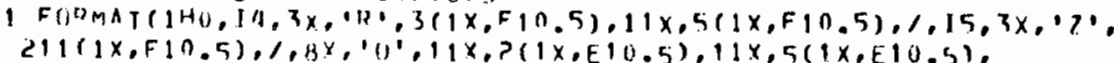

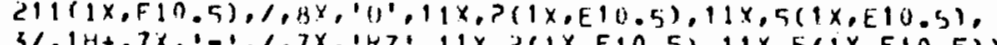
$3 /, 1 H+, 7 x, \cdot-1,1,7 x, 1,7 \cdot, 11 x, 2(1 x, F, n, 5), 11 x, 5(1 x, F, 1 n, 5)$

\section{Flastic INTEgRATION POINTS}

11 DRTNT P14,1E, (SPCRDIIE, IF, 1), חESTRN(IE, IG, 1), DSTRS(TE, IT, 1),

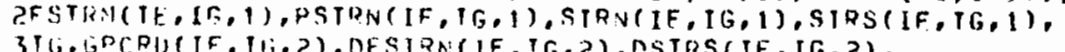

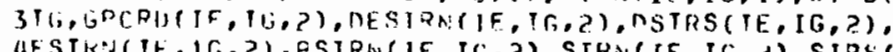

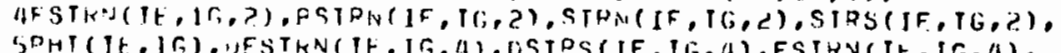

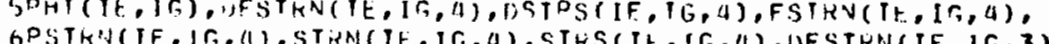

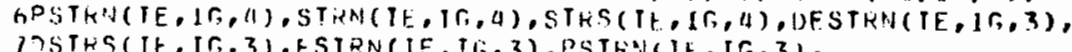

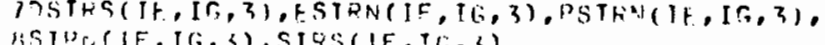

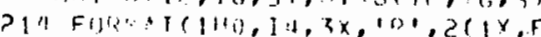

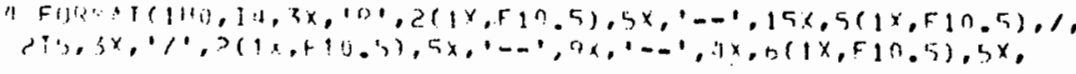


PRINT CIJRRENT PI.ASTIC INIFGRATION POINTS

5ी PRTHT $>03$

203 FODMATCIHO, $1, \cdot$-CURRENT PLASITC POINIS SOINT) $1 ., 1,37(-1)$

TF(n.PPTS.NF.n) GO TO IR

PKIMT PIO

PIO FUPMAT(IHO, ONII I)

riil in lis

19 PRTNT POA, $((1 P P T(1, j), J=1,5), 1=1, N P P T S)$

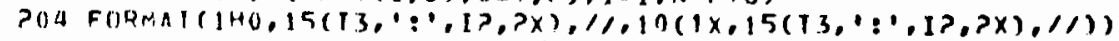

r. PRINT HEARINGS FOR NONE UHANTITTFS

19 IH (IA.T.T.1) PIRTT POR

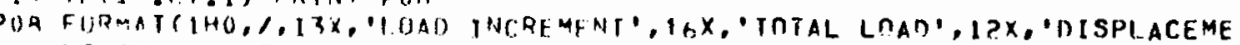

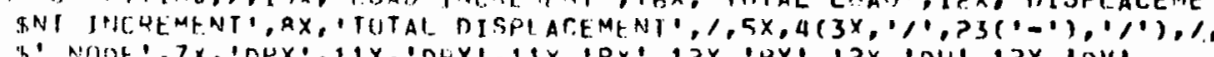

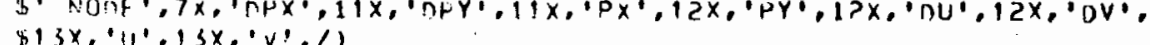

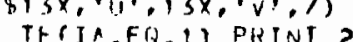

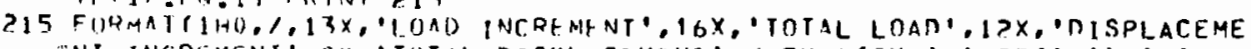

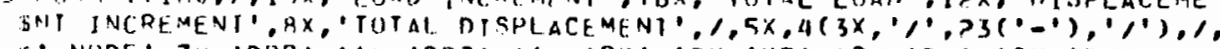

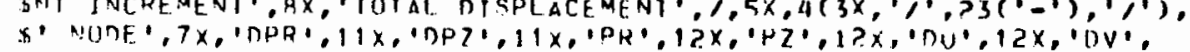

C

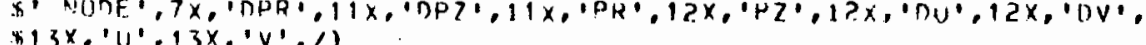

C. PRINT NODF DUANTITJFS FUR OHITPUT REOUFSTEN

C $\begin{gathered}\text { C } \\ \text { C }\end{gathered}$

no) $5 \mathrm{I}=1$, $1 ; \mathrm{N}$

IF (NODEPR(T) .NE. I) GO TO 5

$M R=(1-1) * 2$

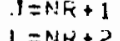

PRIMT $\geq 00, T, \cap P(J), \cap P(L), P(J), P(L), \cap U(J), \cap U(L), U(J), U(L)$

POa FORMAI(1H, IU,A(3X,E11.6))

COMTTNUE

GECK FOR TERMINATING ANAL.YSIS

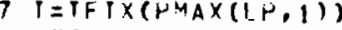

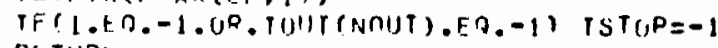

DF. TUPN 
Since the methods of analysis are rather complex and mathematical they cannot conveniently be dealt with in lectures of this nature. Some of the more important methods will, however, be given in brief outline.

(i) Regression : The proportion of zonal trips by each mode is expressed as a function of the system trip and user characteristics, e.g.:

$$
Y=a+B_{1} X_{1}+B_{2} X_{2}+\ldots \ldots \ldots B_{n} X_{1}
$$

Where $x_{1}=\frac{\text { Transit riding time and (Walk and wait and transfer) }}{\text { time. Driving time and terminal time. }}$

$x_{2}=$ Median family income for zone of production.

Zone

of

Production

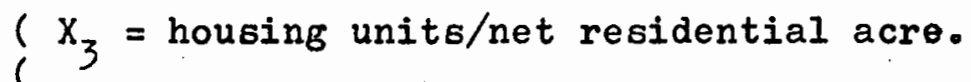

$x_{4}=$ cars/housing unit.

$\left(x_{5}=\right.$ accessibility to employment (transit/car).

$\mathrm{x}_{6}=9 \mathrm{hr}$. parking cost (average rate per hour).

$x_{7}=3 \mathrm{hr}$. parking cost (average rate per hour).

$x_{8}=$ employment/gross acre.

$x_{9}=$ accessibility to population (transit/car)

$Y=$ percentage trips by transit.

(1i) Discriminant Analygis : Develop a linear function of the form $I=a_{1} x_{1}+a_{2} x_{2}+\ldots \ldots \ldots \ldots+a_{n} x_{n}$

which will distinguish between travellers making different mode-choice decisions on the basis of a set of variables $x$ defining the characteristics of the travellers of the alternative choices available to them.

Let $f=\left(a_{1} x_{1}\right)+\left(a_{2} x_{2}\right)$

be a discriminant function.

Where $x_{1}=$ cost difference mode 1 versus mode 2 $x_{2}=$ time difference mode 2 versus mode 1 .

Choose values for $a_{1}$, $a_{2}$ such that $f$ is small for travellers using mode 1 and large for those using mode 2 .

\section{Utility and Preference Analysis :}

Individual preference for travel modes may be estimated by subjecting a set of travellers to a structured series of modal alternatives, defined in terms of varying values of relative cost, time, comfort, etc. and evaluating their "threshold" response (i.e." the point at which a change in a particular modal characteristic would bring about a change in mode choice). Ideally, such an experiment should be based on actual changes in the transportation system; it may be approximated by means of a laboratory or interview experiment. Equivalent analysis may be performed for route choice, ranking modal and route characteristics in order of importance and evaluating response to new forms of transportation. 
since the water resistance had to be taken into account, it was necessary to use a voltage comparator (LM 319) which would output either $5 \mathrm{v}$ or $0 \mathrm{v}$ when the output from the probe itself was $>4,5 \vee$ or $<1 \vee$ respectively. This was compared with the voltage at the comparator created by either resistors R1 and $R 2$ or resistors $R 3$ and $R 4$, depending on which probe was under consideration. The essential difference between the two circuits was that the potential at probe 1 was inverted.

\subsubsection{RC Oscillator}

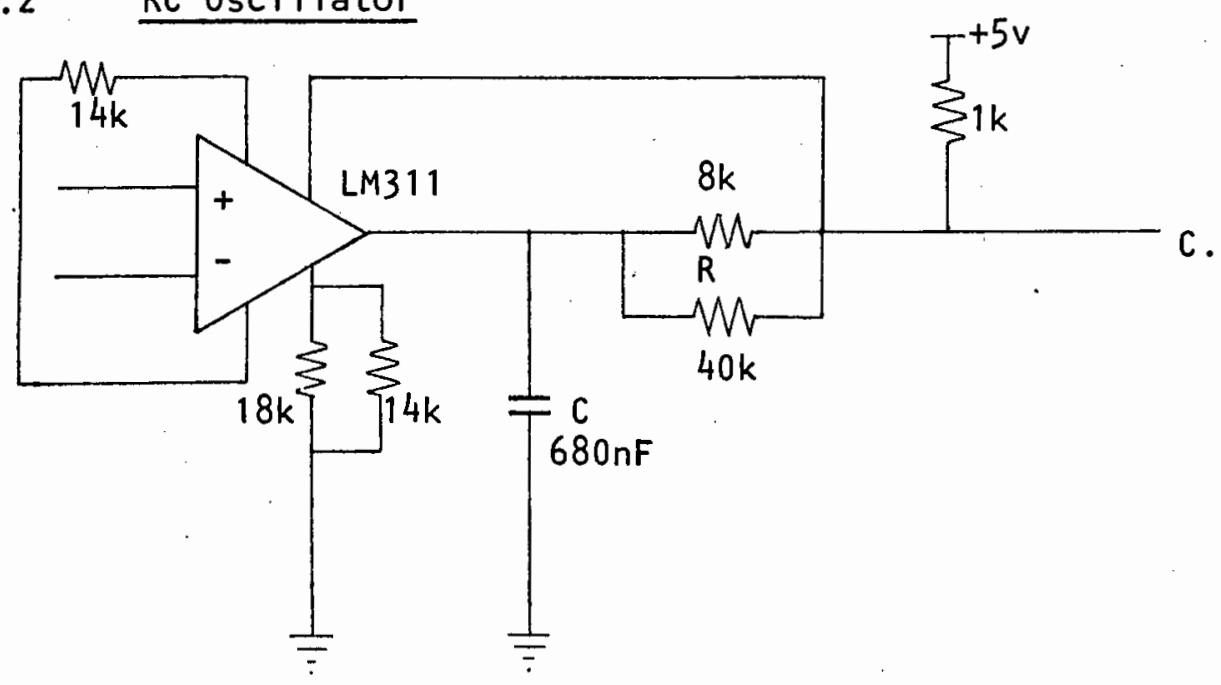

\section{FIGURE 4.5}

The RC oscillator was designed so that the temperature coefficients of $R$ and $C$ cancelled each other out. To achieve this the following components were used:

metal film resistor

- temperature coefficient $=-50 \mathrm{ppm} /{ }^{\circ} \mathrm{C}$

poly-carbonate capacitor - temperature coefficient $=+50 \mathrm{ppm} /{ }^{\circ} \mathrm{C}$

Thus,

$$
\frac{1}{f}=\frac{1}{2 \pi R C}
$$

where
$R$ is resistance in ohms
$C$ is capacitance in far ads
$f$ is frequency 